U.S. Department of the Interior

U.S. Geological Survey

\title{
Physical, Chemical, and Biological Data for Selected Streams in Chester County, Pennsylvania, 1995-97
}

by Andrew G. Reif

Open-File Report 00-238

Prepared in cooperation with the

CHESTER COUNTY WATER RESOURCES AUTHORITY 


\section{U.S. DEPARTMENT OF THE INTERIOR \\ BRUCE BABBITT, Secretary}

U.S. GEOLOGICAL SURVEY

Charles G. Groat, Director

Copies of this report may be purchased from:

U.S. Geological Survey

Branch of Information Services

Box 25286, Building 810

Denver, Colorado 80225-0286
Copies are also available from:

Chester County Water Resources Authority 601 Westtown Road

Suite 270

P.O. Box 2747

Government Services Center

West Chester, Pennsylvania 19380-0990 


\section{CONTENTS}

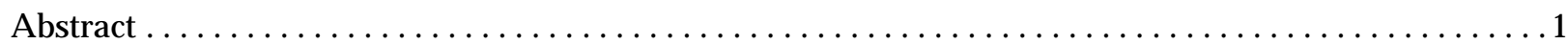

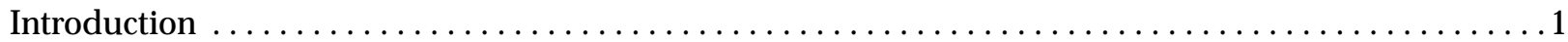

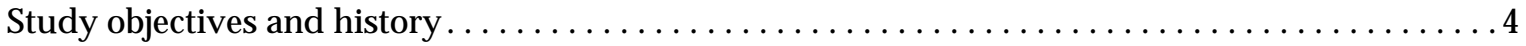

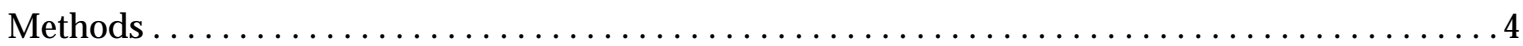

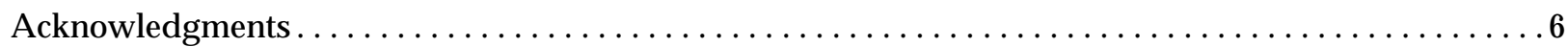

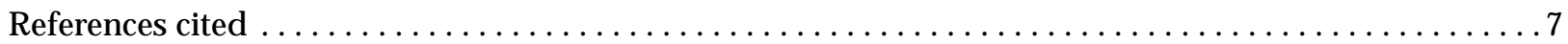

\section{ILLUSTRATION}

Figure 1. Map showing location of sampling sites in Chester County, Pa $\ldots \ldots \ldots \ldots \ldots \ldots \ldots$

\section{TABLES}

Table 1. Sampling sites, station numbers, names, drainage areas, and period of record $\ldots \ldots \ldots \ldots 3$

2. List of taxonomic references used to identify macroinvertebrate samples $\ldots \ldots \ldots \ldots \ldots \ldots$

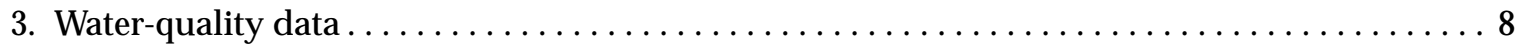

4. Results of stream-bottom sediment sampling .......................... 14

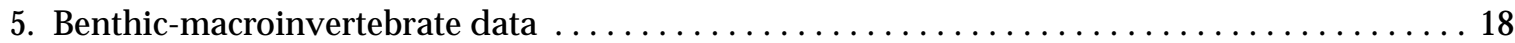

6. Brillouin's diversity index, maximum diversity, minimum diversity, and relative

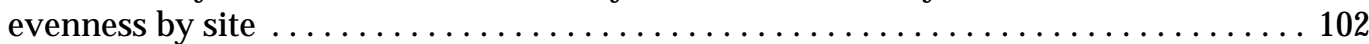

7. Alphabetical checklist of taxa reported from Chester County Biological

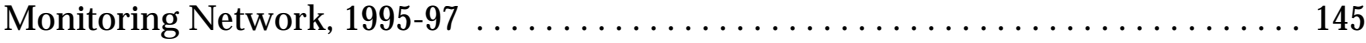

8. Systematic checklist of taxa reported from Chester County Biological

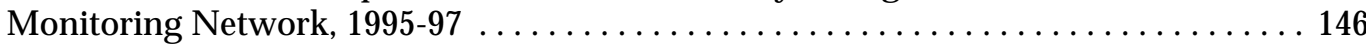




\section{CONVERSION FACTORS AND ABBREVIATED WATER-QUALITY UNITS}

Multiply

inch (in.)

square mile $\left(\mathrm{mi}^{2}\right)$

cubic foot per second $\left(\mathrm{ft}^{3} / \mathrm{s}\right)$

degree Celsius $\left({ }^{\circ} \mathrm{C}\right)$
By

2.54

2.590

${ }^{\circ} \mathrm{F}=1.8\left({ }^{\circ} \mathrm{C}+32\right)$
To obtain

centimeter

square kilometer

cubic meter per second

degree Fahrenheit

Abbreviated water-quality units used in this report:

grams per kilogram $(\mathrm{g} / \mathrm{kg})$

micrograms per gram $(\mu \mathrm{g} / \mathrm{g})$

micrograms per kilogram $(\mu \mathrm{g} / \mathrm{kg})$

microsiemens per centimeter $(\mu \mathrm{S} / \mathrm{cm})$

milligram per liter $(\mathrm{mg} / \mathrm{L})$

millimeter ( $\mathrm{mm})$ 


\title{
PHYSICAL, CHEMICAL, AND BIOLOGICAL DATA FOR SELECTED STREAMS IN CHESTER COUNTY, PENNSYLVANIA, 1995-97
}

\author{
By Andrew G. Reif
}

\begin{abstract}
Physical, chemical, and biological data were collected at 51 sampling sites in Chester County, Pa., from 1970 through 1997 as part of the Stream Conditions of Chester County Program. This report presents data collected from 43 sites from 1995 through 1997 that constitute a continuation of the program. Physical data include water temperature, instantaneous stream discharge, $\mathrm{pH}$, alkalinity, specific conductance, and dissolved oxygen. Chemical data collected include laboratory determinations of nutrients and major ions in whole water samples and selected metals, pesticides, gross polychlorinated biphenyls (PCB's), gross polychlorinated napthalenes ( $\mathrm{PCN}$ 's), and total carbon in stream-sediment samples. The biological data include benthic-macroinvertebrate populations. The data are presented without interpretation.

Chester County is undergoing urbanization as agricultural land is converted to residential developments, commercial areas, and industrial and corporate parks. The major goal of the Stream Conditions of Chester County Program is to further the understanding of stream changes in response to urbanization.

\section{INTRODUCTION}

Chester County, in the southeast corner of Pennsylvania near Philadelphia (fig. 1), is undergoing urbanization as agricultural land is converted to residential developments, commercial areas, and industrial and corporate parks. The Stream Conditions of Chester County Program was developed by the Chester County Water Resources Authority (CCWRA) and the U.S. Geological Survey (USGS) to evaluate stream-water quality and to further the understanding of stream changes in response to urbanization (Lium, 1977, p. 6). This report presents physical, chemical, and biological data collected at 43 sites from 1995 through 1997 as part of the Stream Conditions of Chester County Program (table 1). Physical, chemical, and biological data for the program from 1970 through 1994 were presented previously in two reports. A report by Moore (1989) contains data from 1970 through 1980 and a report by Reif (1999) contains data from 1981 through 1994.

The streams included in the Stream Conditions of Chester County Program drain nearly 95 percent of the county (fig. 1). Valley Creek, Pickering Creek, Stony Run, Pigeon Creek, and French Creek are tributaries to the Schuylkill River. Darby, Crum, Ridley, and Chester Creeks are tributaries to the Delaware River. Buck Run, Doe Run, Indian Run, and Valley Creek are tributaries to the Brandywine Creek. The Red Clay Creek, White Clay Creek, and Brandywine Creek are tributaries to the Christina River, which is a tributary to the Delaware River. Big Elk Creek flows into the Chesapeake Bay. Octoraro Creek is a tributary to the Susquehanna River. All streams sampled originate within the boundaries of Chester County, except for the headwaters of the West Branch Brandywine, French, and Octoraro Creeks.
\end{abstract}




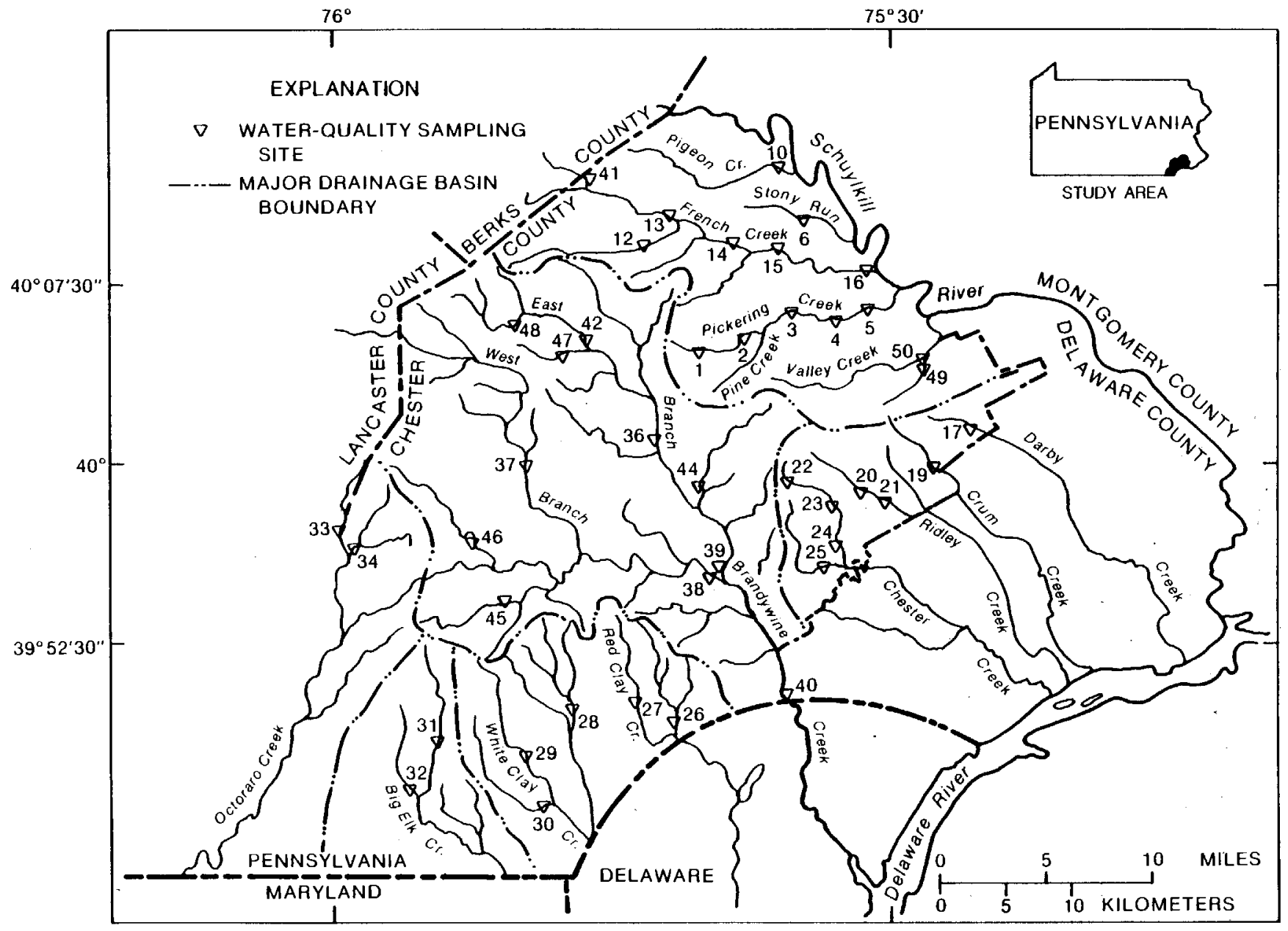

Figure 1. Location of sampling sites in Chester County, Pa. 
Table 1. Sampling sites, station numbers, names, drainage areas, and period of record

\begin{tabular}{|c|c|c|c|c|c|c|}
\hline \multirow{2}{*}{$\begin{array}{c}\text { U.S. } \\
\text { Geological } \\
\text { Survey station } \\
\text { number }\end{array}$} & \multirow{2}{*}{$\begin{array}{l}\text { Site } \\
\text { number }\end{array}$} & \multirow[b]{2}{*}{ Station name } & \multirow{2}{*}{$\begin{array}{l}\text { Drainage } \\
\text { area } \\
\text { (square } \\
\text { miles) }\end{array}$} & \multicolumn{3}{|c|}{ Period of record } \\
\hline & & & & Biological & Chemical & $\begin{array}{l}\text { Bottom } \\
\text { sediments }\end{array}$ \\
\hline 01472080 & 10 & Pigeon Creek near Parker Ford & 12.0 & $1970-97$ & $1969-97$ & 1985,1996 \\
\hline 01472109 & 6 & Stony Run near Spring City & 2.00 & 1970-96 & $1969-96$ & 1985,1996 \\
\hline 01472138 & 13 & French Creek near Coventryville & 19.9 & $1970-97$ & $1969-97$ & 1986,1997 \\
\hline 01472140 & 12 & South Branch French Creek at Coventryville & 12.4 & $1970-97$ & $1969-97$ & 1985,1997 \\
\hline 01472154 & 14 & French Creek near Pughtown & 46.1 & $1970-97$ & $1969-97$ & 1985,1997 \\
\hline 01472157 & 15 & French Creek near Phoenixville & 59.1 & 1970-97 & $1969-97$ & 1994 \\
\hline 014721612 & 16 & French Creek at Railroad Bridge at Phoenixville & 70.7 & 1980-97 & $1970-97$ & 1985,1994 \\
\hline 01472170 & 1 & Pickering Creek near Eagle & 3.09 & $1970-96$ & $1969-96$ & 1987,1996 \\
\hline 01472174 & 2 & Pickering Creek near Chester Springs & 5.98 & 1970-95 & 1969-95 & 1986 \\
\hline 014721854 & 3 & Pickering Creek at Merlin & 21.2 & $1970-97$ & $1969-97$ & 1986,1997 \\
\hline 014721884 & 4 & Pickering Creek at Charlestown Road at Charlestown & 27.5 & $1972-96$ & $1969-96$ & 1985,1996 \\
\hline 01472190 & 5 & Pickering Creek near Phoenixville & 31.4 & $1970-97$ & $1969-97$ & 1986,1994 \\
\hline 01473167 & 49 & Little Valley Creek at Howellville & 6.45 & 1973-96 & $1970-96$ & $1986,87,93$ \\
\hline 01473168 & 50 & Valley Creek near Valley Forge & 12.7 & 1973-96 & $1970-96$ & 1985,1993 \\
\hline 01475300 & 17 & Darby Creek at Waterloo Mills near Devon & 5.15 & 1970-96 & $1969-96$ & 1996 \\
\hline 01475840 & 19 & Crum Creek at Whitehorse & 10.1 & 1970-96 & $1969-96$ & 1986,1996 \\
\hline 01476430 & 20 & Ridley Creek at Goshenville & 4.22 & $1970-96$ & $1969-96$ & 1985 \\
\hline 01476435 & 21 & Ridley Creek at Dutton Mill near West Chester & 9.71 & $1970-96$ & $1969-96$ & 1986 \\
\hline 01476790 & 22 & East Branch Chester Creek at Green Hill & .63 & $1970-97$ & $1969-97$ & 1986,1997 \\
\hline 01476830 & 23 & East Branch Chester Creek at Milltown & 5.77 & 1970-96 & $1969-96$ & 1986,1996 \\
\hline 01476835 & 24 & East Branch Chester Creek at Westtown & 10.4 & 1970-97 & $1969-97$ & 1985,1994 \\
\hline 01476840 & 25 & $\begin{array}{l}\text { Goose Creek tributary to East Branch Chester Creek near } \\
\text { West Chester }\end{array}$ & 4.28 & $\begin{array}{l}1975-82 \\
1988-97\end{array}$ & $\begin{array}{l}1970-82 \\
1988-97\end{array}$ & 1988,1993 \\
\hline 01476848 & 51 & $\begin{array}{l}\text { East Branch Chester Creek below Goose Creek near } \\
\text { West Chester }\end{array}$ & 19.2 & $1983-97$ & $1970-97$ & 1986,1994 \\
\hline 01478120 & 28 & East Branch White Clay Creek at Avondale & 11.3 & $1970-97$ & $1970-97$ & 1985,1993 \\
\hline 01478190 & 29 & Middle Branch White Clay Creek at Wickerton & 9.94 & $1970-97$ & $1970-97$ & 1986,1993 \\
\hline 01478220 & 30 & West Branch White Clay Creek near Chesterville & 9.92 & 1970-97 & $1970-97$ & 1985,1993 \\
\hline 01479680 & 27 & West Branch Red Clay Creek at Kennett Square & 9.79 & 1970-97 & $1970-97$ & $1983,86,93$ \\
\hline 01479800 & 26 & East Branch Red Clay Creek near Five Point & 10.2 & 1970-97 & $1970-97$ & 1985,1993 \\
\hline 01480434 & 37 & West Branch Brandywine Creek at Rock Run & 37.3 & $1970-96$ & $1970-96$ & - \\
\hline 01480629 & 46 & Buck Run at Doe Run & 22.6 & 1973-97 & $1971-97$ & 1985,1995 \\
\hline 01480632 & 45 & Doe Run at Springdell & 11.8 & 1973-97 & $1971-97$ & 1986,1995 \\
\hline 01480640 & 38 & West Branch Brandywine Creek at Wawaset & 134 & 1970-97 & $1970-97$ & 1985,1993 \\
\hline 01480648 & 48 & East Branch Brandywine Creek near Cupola & 5.98 & 1973-95 & 1971-95 & 1986,1995 \\
\hline 01480653 & 42 & East Branch Brandywine Creek at Glenmoore & 16.5 & 1973-95 & 1971-95 & 1985,1995 \\
\hline 01480656 & 47 & Indian Run near Springton & 4.26 & 1974-95 & 1971-95 & 1986,1995 \\
\hline 01480700 & 36 & East Branch Brandywine Creek near Downingtown & 60.6 & 1970-96 & $1970-96$ & 1985 \\
\hline 01480903 & 44 & Valley Creek at Mullsteins Meadows near Downingtown & 16.1 & 1973-97 & $1971-97$ & 1985,1995 \\
\hline 01480950 & 39 & East Branch Brandywine Creek at Wawaset & 123 & 1979-97 & $1970-97$ & 1986,1993 \\
\hline 01481030 & 40 & Brandywine Creek near Chadds Ford & 291 & $1972-97$ & $1970-97$ & 1985,1997 \\
\hline 01494900 & 31 & East Branch Big Elk Creek at Elkview & 11.1 & $1970-97$ & $1970-97$ & 1986,1994 \\
\hline 01494950 & 32 & West Branch Big Elk Creek near Oxford & 10.0 & $1970-96$ & $1970-96$ & 1985,1994 \\
\hline 01578340 & 33 & East Branch Octoraro Creek at Christiana & 11.8 & 1970-97 & $1970-97$ & 1994 \\
\hline 01578343 & 34 & Valley Creek near Atglen & 10.5 & $1970-96$ & $1970-96$ & 1985 \\
\hline
\end{tabular}




\section{Study Objectives and History}

The major goal of the Stream Conditions of Chester County Program is to assess the water quality of streams in the county and to further the understanding of stream changes in response to urbanization. The physical, chemical, and biological data presented in this report were collected in support of this goal.

The investigation began in the fall of 1969 with a reconnaissance of the county to determine the general conditions of streams and land-use patterns. In 1970, the reconnaissance served as a guide to establish a chemical and biological water-quality network of 40 sites in 13 stream basins. The sites were established on the basis of equal cumulative square miles of drainage area within the basin (Lium, 1977). The sites were established away from any known source of pollution so that the water quality of the overall stream could be assessed. During 1970-72, samples were collected in the spring and fall. In 1971, 10 new stations were added to the chemical sampling program. In 1973, these 10 stations were added to the biological sampling program and sampling was reduced to once a year in the fall. In 1979, qualitative sampling was replaced by quantitative sampling at sites 16 and 39. In 1982, nine stations (sites 7, 8, 9, 11, $18,25,35,41$, and 43) were dropped from the network, and in 1983, one station (site 51) was added. In 1988 site 25 was reestablished, bringing the current number of active stations to 43 .

\section{Methods}

Biological samples consisted of benthic macroinvertebrates collected from a riffle area. During each visit, benthic macroinvertebrates were sampled by collecting 10 rocks ( $45-90 \mathrm{~mm}$ in diameter) at random (Lium, 1974). All invertebrates from the rocks were composited in a container and stored in 70 percent isopropyl alcohol for later identification. A complete description of the sampling technique is described in a report by Moore $(1987$, p. 7). Benthic-macroinvertebrate samples were analyzed at the USGS office in Malvern, Pa. Benthic macroinvertebrates were identified to the lowest taxonomic level possible. A voucher collection of organisms identified resides at the USGS, Water Resources Division, Malvern, Pa. A list of taxonomic references that the identifications were based on is given in table 2. Brillouin's diversity index, maximum diversity, minimum diversity, and evenness were calculated for each benthic-

macroinvertebrate sample (table 6). Mean and median Brillouin's diversity index along with the standard deviation and standard error of the mean were calculated for each site (table 7). Brillouin's diversity index is calculated by the following formula:

$$
H=(C / N) \log _{10}\left(N ! / N_{1} ! N_{2} ! \ldots . . N_{s} !\right)
$$

$$
\begin{aligned}
\text { where } \mathrm{H} & =\text { diversity, } \\
\mathrm{C} & =3.3219, \\
\mathrm{~N} & =\text { total number of individuals, } \\
\mathrm{s} & =\text { number of taxa, and } \\
\mathrm{N}_{\mathrm{i}}(\mathrm{i} & =1,2, \ldots ., \mathrm{s})=\text { number of individuals in the } \mathrm{i}^{\text {th }} \text { taxa }
\end{aligned}
$$


Table 2. List of taxonomic references used to identify macroinvertebrate samples

\begin{tabular}{ll}
\hline \multicolumn{1}{c}{ Taxonomic Group } & \multicolumn{1}{c}{ Reference } \\
\hline Turbellaria & Pennak, 1989 \\
Nemertea & Pennak, 1989 \\
Nematoda & Pennak, 1989 \\
Gastropoda & Harman and Berg, 1971 \\
Bivalvia & Peckarsky and others, 1990 \\
Annelida & Pennak, 1989 \\
Crustacea & Pennak, 1989 \\
Arachnida & Pennak, 1989 \\
Ephemeroptera & Peckarsky and others, 1990, Merritt and Cummins, 1996 \\
Odonata & Peckarsky and others, 1990, Merritt and Cummins, 1996 \\
Plecoptera & Peckarsky and others, 1990, Merritt and Cummins, 1996 \\
Megaloptera & Peckarsky and others, 1990, Merritt and Cummins, 1996 \\
Neuroptera & Peckarsky and others, 1990, Merritt and Cummins, 1996 \\
Lepidoptera & Peckarsky and others, 1990, Merritt and Cummins, 1996 \\
Trichoptera & Wiggins, 1996 \\
Diptera & Peckarsky and others, 1990, Merritt and Cummins, 1996 \\
Hymenoptera & Brigham and others,1982, Merritt and Cummins, 1996 \\
Coleoptera & Brown, 1976, Peckarsky and others, 1990 \\
\hline
\end{tabular}

Brillouin's diversity index is based on the different kinds of organisms (taxa) present in a community and their relative abundances. Diversity is high if a community has many taxa and their abundances are evenly distributed; diversity is low if the taxa are few and their abundances are unevenly distributed (Moore, 1987). Brillouin's diversity can range from zero to infinity but usually is below 5.0. Brillouin's diversity values below 1.0 are associated with waters receiving heavy levels of organic wastes. Brillouin's diversity values between 1.0 and 3.0 are associated with waters receiving moderate levels of organic wastes, and Brillouin's diversity values between 3.0 and 5.0 are associated with waters receiving little or no organic wastes (Wilhm and Dorris, 1968; Wilhm, 1970). Brillouin's diversity measures the effect of community stress and not pollution directly. Community stress may be the result of many factors, including organic or toxic pollution, physical stress, or lack of suitable habitat.

Other information can be obtained from the number of taxa present in a community and their relative abundances. The maximum diversity $\left(\mathrm{H}_{\max }\right)$ exists when all individuals are distributed as evenly as possible among the taxa. The minimum diversity $\left(\mathrm{H}_{\mathrm{min}}\right)$ exists when all individuals are distributed as unevenly as possible among the taxa. Evenness (e) describes the observed degree of uniformity of the distribution of individuals among the taxa in the collection. Evenness values between 0.5 and 1.0 generally indicate a balanced community (Moore, 1987). The maximum diversity $\left(\mathrm{H}_{\max }\right.$, minimum diversity $\left(\mathrm{H}_{\min }\right)$, and evenness (e) are calculated by the following formulas:

$$
\begin{aligned}
& \mathrm{H}_{\max }=\mathrm{C} / \mathrm{N}\left\{\log _{10}(\mathrm{~N} !)-\mathrm{s}\left(\log _{10}\right)[(\mathrm{N} / \mathrm{s}) !]\right\} \\
& \mathrm{H}_{\min }=\mathrm{C} / \mathrm{N}\left(\log _{10}(\mathrm{~N} !)-\log _{10}\{[\mathrm{~N}-(\mathrm{s}-1)] !\}\right) \\
& \mathrm{e}=\mathrm{H}-\mathrm{H}_{\min } / \mathrm{H}_{\max }-\mathrm{H}_{\min }
\end{aligned}
$$

Surface-water samples for chemical analysis were collected in conjunction with the biological samples by use of techniques described by Brown and others (1970, p. 5). Chemical samples were analyzed at the USGS National Water Quality Laboratory in Arvada, Colo. Whole water samples were analyzed for nutrients, and chloride. Nutrients analyzed and reported from whole water samples were nitrite, nitrate, ammonia, and phosphorus. 
Stream-bottom sediment samples were collected by hand from the top 6 to $12 \mathrm{in}$. of sediment with a polyethylene scoop and sieved through a $2-\mathrm{mm}$ polyethylene sieve to remove gravel. The sediment was washed with native water and collected in a polyethylene collection basin. The samples were

homogenized and then transferred to clean glass or polyethylene containers and placed on ice for shipment to the USGS National Water Quality Lab. Stream-bottom sediment samples were analyzed for selected metals, pesticides, gross polychlorinated biphenyls ( $\left.\mathrm{PCB}^{\prime} \mathrm{s}\right)$, gross polychlorinated napthalenes (PCN's), and total carbon. Selected metals analyzed and reported from stream-bottom sediment samples were arsenic, cadmium, chromium, copper, iron, lead, manganese, mercury, and zinc. Selected pesticides analyzed and reported from stream-bottom sediment samples were aldrin, chlordane, DDD, DDE, DDT, dieldrin, endosulfan, endrin, heptachlor, heptachlor epoxide, lindane, methoxychlor, mirex, perthane, and toxaphene.

\section{ACKNOWLEDGMENTS}

The cooperation of the Chester County Board of Commissioners, the Chester County Water Resources Authority, Chester County Planning Commission, and the Chester County Health Department is gratefully acknowledged. Special thanks are extended to David C. Yaeck and Irene B. Brooks, former Executive Directors of the Chester County Water Resources Authority, for their interest and support throughout the program's history. The author also would like to thank Dr. Richard McLean for his assistance throughout the years. The author also recognizes Michael D. Bilger and J. Kent Crawford (U.S. Geological Survey) for their review of this report. Technical support was provided by Kim Otto and Joanne Koch (U.S. Geological Survey). 


\section{REFERENCES CITED}

Brigham, A.R., Brigham, W.U., and Gnilka, A., eds., 1982, Aquatic insects and oligochaetes of North and South Carolina: Mahomet, Ill., Midwest Aquatic Enterprises, 837 p.

Brown, Eugene, Skougstad, M.W., and Fishman, M.J., 1970, Methods for collection and analysis of water samples for dissolved minerals and gases: U.S. Geological Survey Techniques of Water-Resources Investigations, book 5, chap. A1, $160 \mathrm{p}$.

Brown, H.P., 1976, Aquatic dryopoid beetles (Coleoptera) of the United States: U.S. Environmental Protection Agency, Water Pollution Control Research Series 18050 ELD04/72, 82 p.

Harman, W.N., and Berg, C.O., 1971, The freshwater snails of central New York with illustrated keys to the genera and species: Search Agriculture, v. 1, no. 4, 68 p.

Lium, B.W., 1974, Some biological aspects of pools and riffles in gravel bed streams in Western United States: U.S. Geological Journal of Research, v. 2, no. 3, p. 379-384.

1977, Limnological studies of the major streams in Chester County, Pennsylvania: U.S. Geological Survey Open-File Report 77-462, 37 p.

Merritt, R.W., and Cummins, K.W., eds., 1996, An introduction to the aquatic insects of North America, (3d ed.): Dubuque, Iowa, Kendall Hunt Publishing Company, 862 p.

Moore, C.R., 1987, Determination of benthic-invertebrate indices and water-quality trends of selected streams in Chester County, Pennsylvania, 1969-80: U.S. Geological Survey Water-Resources Investigations Report 85-4177, 62 p.

1989, Physical, chemical, and biological data for selected streams in Chester County, Pennsylvania, 1969-80: U.S. Geological Survey Open-File Report 85-686, 289 p.

Peckarsky, B.L., Fraissinet, P.R., Penton, M.A., and Conklin, D.J., Jr., 1990, Freshwater macroinvertebrates of Northeastern North America: Ithaca, N.Y., Cornell University Press, 442 p.

Pennak, R.W., 1989, Fresh-water invertebrates of the United States, (3d ed.): New York, John Wiley and Sons, $803 \mathrm{p}$.

Reif, A.G., 1999, Physical, chemical, and biological data for selected streams in Chester County, Pennsylvania, 1981-94: U.S. Geological Survey Open-File Report 99-216, 607 p.

Wiggins, G.B., 1996, Larvae of the North American caddisfly genera (Trichoptera), (2d ed.): Toronto, University of Toronto Press, $457 \mathrm{p}$.

Wilhm, J.L., 1970, Range of diversity index in benthic macro-invertebrate populations: Water Pollution Control Federation Journal, v. 42, no. 5, p. R221-R251.

Wilhm, J., and Dorris, T., 1968, Biological parameters for water quality criteria: Bioscience, v. 18, p. 477481. 
Table 3. Water-quality data

$[\mu \mathrm{S} / \mathrm{cm}$, microsiemens per centimeter at 25 degrees Celsius; mg/L, milligrams per liter, <, less than; --, no data]

\begin{tabular}{|c|c|c|c|c|c|c|c|}
\hline Date & $\begin{array}{c}\text { Agency } \\
\text { collecting } \\
\text { sample } \\
\text { (code number) } \\
(00027)\end{array}$ & $\begin{array}{c}\text { Agency } \\
\text { analyzing } \\
\text { sample } \\
\text { (code number) } \\
(00028)\end{array}$ & $\begin{array}{l}\text { Instantaneous } \\
\text { discharge } \\
\text { (cubic feet per } \\
\text { second) } \\
(00061)\end{array}$ & $\begin{array}{c}\text { Specific } \\
\text { conductance } \\
(\mu \mathrm{S} / \mathrm{cm}) \\
(00095)\end{array}$ & $\begin{array}{c}\mathrm{pH}, \text { water, } \\
\text { whole field } \\
\text { (standard units) } \\
(00400)\end{array}$ & $\begin{array}{c}\text { Water } \\
\text { temperature } \\
\text { (degrees } \\
\text { Celsius) } \\
(00010)\end{array}$ & $\begin{array}{c}\text { Dissolved } \\
\text { oxygen } \\
(\mathrm{mg} / \mathrm{L}) \\
(00300)\end{array}$ \\
\hline \multicolumn{8}{|c|}{01472080 (site 10) Pigeon Creek near Parker Ford, Pa. (Lat 4012 03n Long 075 37 10w) } \\
\hline $11 / 22 / 95$ & 1028 & 80020 & 11 & 177 & 6.9 & 5.5 & 12.9 \\
\hline $11 / 06 / 96$ & 1028 & 80020 & 13 & 166 & 6.3 & 10.0 & 12.4 \\
\hline $10 / 30 / 97$ & 1028 & 80020 & 3.2 & 182 & 6.9 & 8.0 & 11.7 \\
\hline \multicolumn{8}{|c|}{01472109 (site 6) Stony Run near Spring City, Pa. (Lat 4010 11n Long 07534 45w) } \\
\hline $11 / 13 / 95$ & 1028 & 80020 & 2.1 & 239 & 7.0 & 5.5 & 12.0 \\
\hline $11 / 06 / 96$ & 1028 & 80020 & 2.3 & 239 & 6.4 & 9.5 & 11.2 \\
\hline \multicolumn{8}{|c|}{01472138 (site 13) French Creek near Coventryville, Pa. (Lat 4010 14n Long 075 41 50w) } \\
\hline $11 / 06 / 95$ & 1028 & 80020 & 14 & 134 & 7.1 & 5.5 & 12.1 \\
\hline $10 / 01 / 97$ & 1028 & 80020 & 5.7 & 142 & 6.8 & 13.5 & 9.1 \\
\hline \multicolumn{8}{|c|}{01472140 (site 12) South Branch French Creek at Coventryville, Pa. (Lat 4009 18n Long 075 42 52w) } \\
\hline $11 / 06 / 95$ & 1028 & 80020 & 8.4 & 201 & 7.1 & 6.0 & 13.4 \\
\hline $10 / 09 / 97$ & 1028 & 80020 & 3.7 & 203 & 7.3 & 16.0 & 11.0 \\
\hline \multicolumn{8}{|c|}{01472154 (site 14) French Creek near Pughtown, Pa. (Lat 4009 17n Long 07538 25w) } \\
\hline $11 / 06 / 95$ & 1028 & 80020 & 29 & 165 & 7.4 & 6.5 & 12.3 \\
\hline $10 / 08 / 97$ & 1028 & 80020 & 9.3 & 171 & 6.9 & 16.5 & 9.5 \\
\hline \multicolumn{8}{|c|}{01472157 (site 15) French Creek near Phoenixville, Pa. (Lat 4009 05n Long 07536 06w) } \\
\hline $10 / 27 / 95$ & 1028 & 80020 & 45 & 172 & 7.4 & 9.5 & 11.3 \\
\hline $10 / 28 / 96$ & 1028 & 80020 & 99 & 148 & 6.8 & 14.0 & 10.1 \\
\hline $10 / 02 / 97$ & 1028 & 80020 & 21 & 166 & 7.3 & 11.5 & 11.4 \\
\hline \multicolumn{8}{|c|}{014721612 (site 16) French Creek at Railroad Bridge at Phoenixville, Pa.(Lat 4008 10n Long 07530 41w) } \\
\hline $11 / 07 / 95$ & 1028 & 80020 & 47 & 225 & 7.6 & 5.5 & 12.1 \\
\hline $10 / 02 / 97$ & 1028 & 80020 & 22 & 231 & 7.2 & 11.5 & 10.2 \\
\hline \multicolumn{8}{|c|}{01472170 (site 1) Pickering Creek near Eagle, Pa. (Lat 4004 43n Long 07539 14w) } \\
\hline $10 / 12 / 95$ & 1028 & 80020 & .82 & 250 & 7.5 & 17.0 & 10.2 \\
\hline $10 / 31 / 96$ & 1028 & 80020 & 3.9 & 218 & 6.8 & 11.5 & 10.6 \\
\hline \multicolumn{8}{|c|}{01472174 (site 2) Pickering Creek near Chester Springs, Pa. (Lat 4005 22n Long 075 37 50w) } \\
\hline $10 / 12 / 95$ & 1028 & 80020 & 2.3 & 228 & 7.6 & 15.0 & 9.6 \\
\hline \multicolumn{8}{|c|}{014721854 (site 3) Pickering Creek at Merlin, Pa. (Lat 4006 25n Long 07535 34w) } \\
\hline $10 / 12 / 95$ & 1028 & 80020 & 7.1 & 230 & 7.6 & 13.0 & 10.0 \\
\hline $10 / 16 / 97$ & 1028 & 80020 & 9.3 & 228 & 6.9 & 12.5 & 9.4 \\
\hline \multicolumn{8}{|c|}{014721884 (site 4) Pickering Creek at Charlestown Rd at Charlestown, Pa. (Lat 4005 57n Long 07533 20w) } \\
\hline $10 / 11 / 95$ & 1028 & 80020 & 9.7 & 250 & 7.8 & 16.0 & 11.6 \\
\hline $10 / 02 / 96$ & 1028 & 80020 & 33 & 221 & 6.6 & 14.5 & 10.2 \\
\hline \multicolumn{8}{|c|}{01472190 (site 5) Pickering Creek near Phoenixville, Pa. (Lat 4006 33n Long 075 31 42w) } \\
\hline $10 / 11 / 95$ & 1028 & 80020 & 11 & 248 & 7.7 & 13.5 & 10.9 \\
\hline $10 / 31 / 96$ & 1028 & 80020 & 41 & 228 & 6.7 & 10.5 & 11.4 \\
\hline $10 / 30 / 97$ & 1028 & 80020 & 14 & 249 & 7.1 & 6.0 & 10.9 \\
\hline \multicolumn{8}{|c|}{01473167 (site 49) Little Valley Creek at Howellville, Pa. (Lat 4004 00n Long 075 28 22w) } \\
\hline $10 / 30 / 95$ & 1028 & 80020. & 6.4 & 613 & 7.9 & 9.5 & 11.4 \\
\hline $10 / 24 / 96$ & 1028 & 80020 & 12 & 576 & 7.3 & 12.5 & 10.3 \\
\hline \multicolumn{8}{|c|}{01473168 (site 50) Valley Creek near Valley Forge, Pa. (Lat 4004 11n Long 07528 25w) } \\
\hline $10 / 30 / 95$ & 1028 & 80020 & 13 & 594 & 8.2 & 10.0 & 11.7 \\
\hline $10 / 24 / 96$ & 1028 & 80020 & 27 & 595 & 7.6 & 12.5 & 10.6 \\
\hline \multicolumn{8}{|c|}{01475300 (site 17) Darby Creek at Waterloo Mills near Devon, Pa. (Lat 4001 21n Long 075 25 20w) } \\
\hline $10 / 19 / 95$ & 1028 & 80020 & 3.0 & 301 & 7.4 & 11.5 & 10.0 \\
\hline $10 / 16 / 96$ & 1028 & 80020 & 5.6 & 296 & 6.5 & 11.5 & 10.4 \\
\hline
\end{tabular}


Table 3. Water-quality data-Continued

\begin{tabular}{|c|c|c|c|c|c|c|}
\hline Date & $\begin{array}{c}\text { Alkalinity } \\
\text { whole water } \\
\text { total, field } \\
\left(\mathrm{mg} / \mathrm{L} \text { as } \mathrm{CaCO}_{3}\right) \\
(00419)\end{array}$ & $\begin{array}{l}\text { Dissolved } \\
\text { chloride } \\
\text { (mg/L as Cl) } \\
(00940)\end{array}$ & $\begin{array}{l}\text { Dissolved nitrite } \\
\text { nitrogen } \\
(\mathrm{mg} / \mathrm{L} \text { as } \mathrm{N}) \\
(00613)\end{array}$ & $\begin{array}{c}\text { Dissolved } \\
\mathrm{NO}_{2}+\mathrm{NO}_{3} \\
\text { nitrogen } \\
(\mathrm{mg} / \mathrm{L} \text { as } \mathrm{N}) \\
(00631)\end{array}$ & $\begin{array}{c}\text { Dissolved ammonia } \\
\text { nitrogen } \\
(\mathrm{mg} / \mathrm{L} \text { as } \mathrm{N}) \\
(00608)\end{array}$ & $\begin{array}{c}\text { Dissolved } \\
\text { orthophosphorus } \\
\text { (mg/L as } \mathrm{P}) \\
(00671)\end{array}$ \\
\hline \multicolumn{7}{|c|}{01472080 (site 10) Pigeon Creek near Parker Ford, Pa. (Lat 4012 03n Long 07537 10w) } \\
\hline $11 / 22 / 95$ & 30 & 12 & 0.020 & 2.80 & $<0.015$ & 0.020 \\
\hline $11 / 06 / 96$ & 38 & 12 & .030 & 3.00 & $<.015$ & $<.010$ \\
\hline $10 / 30 / 97$ & 43 & 11 & $<.010$ & 1.81 & $<.015$ & .045 \\
\hline \multicolumn{7}{|c|}{01472109 (site 6) Stony Run near Spring City, Pa. (Lat 4010 11n Long 07534 45w) } \\
\hline $11 / 13 / 95$ & 33 & 17 & .030 & 5.20 & .030 & .070 \\
\hline $11 / 06 / 96$ & 35 & 20 & .030 & 6.50 & $<.015$ & .020 \\
\hline \multicolumn{7}{|c|}{01472138 (site 13) French Creek near Coventryville, Pa. (Lat 4010 14n Long 075 41 50w) } \\
\hline $11 / 06 / 95$ & 29 & 8.4 & $<.010$ & .660 & $<.015$ & $<.010$ \\
\hline $10 / 01 / 97$ & 39 & 8.4 & $<.010$ & .595 & $<.015$ & .010 \\
\hline \multicolumn{7}{|c|}{01472140 (site 12) South Branch French Creek at Coventryville, Pa. (Lat 40 09 18n Long 07542 52w) } \\
\hline $11 / 06 / 95$ & 59 & 14 & .010 & 2.70 & $<.015$ & $<.010$ \\
\hline $10 / 09 / 97$ & 96 & 14 & .010 & 1.97 & $<.015$ & $<.010$ \\
\hline \multicolumn{7}{|c|}{01472154 (site 14) French Creek near Pughtown, Pa. (Lat 4009 17n Long 07538 25w) } \\
\hline $11 / 06 / 95$ & 41 & 11 & .020 & 1.20 & $<.015$ & $<.010$ \\
\hline $10 / 08 / 97$ & 75 & 11 & $<.010$ & .919 & $<.015$ & $<.010$ \\
\hline \multicolumn{7}{|c|}{01472157 (site 15) French Creek near Phoenixville, Pa. (Lat 4009 05n Long 075 36 06w) } \\
\hline $10 / 27 / 95$ & 34 & 11 & $<.010$ & 1.20 & $<.015$ & .020 \\
\hline $10 / 28 / 96$ & 82 & 10 & .010 & 1.60 & $<.015$ & $<.010$ \\
\hline $10 / 02 / 97$ & 43 & 11 & .012 & 1.12 & $<.015$ & $<.010$ \\
\hline \multicolumn{7}{|c|}{014721612 (site 16) French Br Creek at Railroad Bridge at Phoenixville, Pa. (Lat 4008 10n Long 07530 41w) } \\
\hline $11 / 07 / 95$ & 50 & 16 & .010 & 1.60 & $<.015$ & $<.010$ \\
\hline $10 / 02 / 97$ & 59 & 15 & .019 & 1.13 & $<.015$ & $<.010$ \\
\hline \multicolumn{7}{|c|}{01472170 (site 1) Pickering Creek near Eagle, Pa. (Lat 4004 43n Long 07539 14w) } \\
\hline $10 / 12 / 95$ & 44 & 25 & $<.010$ & 1.80 & $<.015$ & .010 \\
\hline $10 / 31 / 96$ & 36 & 23 & .010 & 2.40 & $<.015$ & $<.010$ \\
\hline \multicolumn{7}{|c|}{01472174 (site 2) Pickering Creek near Chester Springs, Pa. (Lat 4005 22n Long 07537 50w) } \\
\hline $10 / 12 / 95$ & 46 & 17 & $<.010$ & 1.80 & $<.015$ & .010 \\
\hline \multicolumn{7}{|c|}{014721854 (site 3) Pickering Creek at Merlin, Pa. (Lat 4006 25n Long 07535 34w) } \\
\hline $10 / 12 / 95$ & 46 & 21 & $<.010$ & 1.30 & $<.015$ & .020 \\
\hline $10 / 16 / 97$ & 75 & 21 & $<.010$ & 1.18 & $<.015$ & .015 \\
\hline \multicolumn{7}{|c|}{014721884 (site 4) Pickering Creek at Charlestown Rd Br at Charlestown, Pa. (Lat 4005 57n Long 07533 20w) } \\
\hline $10 / 11 / 95$ & 55 & 22 & $<.010$ & 1.20 & $<.015$ & .010 \\
\hline $10 / 02 / 96$ & 39 & 22 & $<.010$ & 1.90 & .020 & .010 \\
\hline \multicolumn{7}{|c|}{01472190 (site 5) Pickering Creek near Phoenixville, Pa. (Lat 4006 33n Long 07531 42w) } \\
\hline $10 / 11 / 95$ & 28 & 22 & $<.010$ & 1.10 & .015 & .020 \\
\hline $10 / 31 / 96$ & 55 & 22 & .010 & 1.80 & $<.015$ & $<.010$ \\
\hline $10 / 30 / 97$ & 79 & 24 & $<.010$ & 1.14 & $<.020$ & $<.010$ \\
\hline \multicolumn{7}{|c|}{ 01473167(site 49) Little Valley Creek at Howellville, Pa. (Lat 4004 00n Long 075 28 22w) } \\
\hline $10 / 30 / 95$ & 154 & 58 & $<.010$ & 1.80 & $<.015$ & .020 \\
\hline $10 / 24 / 96$ & 170 & 63 & .010 & 2.60 & .030 & .020 \\
\hline \multicolumn{7}{|c|}{01473168 (site 50) Valley Creek near Valley Forge, Pa. (Lat 4004 11n Long 07528 25w) } \\
\hline $10 / 30 / 95$ & 184 & 41 & $<.010$ & 2.10 & .020 & .020 \\
\hline $10 / 24 / 96$ & 221 & 44 & .030 & 2.50 & .100 & .030 \\
\hline \multicolumn{7}{|c|}{01475300 (site 17) Darby Creek at Waterloo Mills near Devon, Pa. (Lat 4001 21n Long 07525 20w) } \\
\hline $10 / 19 / 95$ & 63 & 27 & .010 & 1.30 & $<.015$ & .020 \\
\hline $10 / 16 / 96$ & 68 & 29 & .020 & 2.20 & .070 & .010 \\
\hline
\end{tabular}


Table 3. Water-quality data-Continued

$[\mu \mathrm{S} / \mathrm{cm}$, microsiemens per centimeter at 25 degrees Celsius; mg/L, milligrams per liter, <, less than; --, no data]

\begin{tabular}{|c|c|c|c|c|c|c|c|}
\hline Date & $\begin{array}{c}\text { Agency } \\
\text { collecting } \\
\text { sample } \\
\text { (code number) } \\
\text { (00027) }\end{array}$ & $\begin{array}{c}\text { Agency } \\
\text { analyzing } \\
\text { sample } \\
\text { (code number) } \\
\text { (00028) }\end{array}$ & $\begin{array}{l}\text { Instantaneous } \\
\text { discharge } \\
\text { (cubic feet per } \\
\text { second) } \\
\text { (00061) }\end{array}$ & $\begin{array}{c}\text { Specific } \\
\text { conductance } \\
(\mu \mathrm{S} / \mathrm{cm}) \\
(00095)\end{array}$ & $\begin{array}{c}\mathrm{pH}, \text { water, } \\
\text { whole field } \\
\text { (standard units) } \\
(00400)\end{array}$ & $\begin{array}{l}\text { Water } \\
\text { temperature } \\
\text { (degrees } \\
\text { Celsius) } \\
(00010)\end{array}$ & $\begin{array}{c}\text { Dissolved } \\
\text { oxygen } \\
(\mathrm{mg} / \mathrm{L}) \\
(00300)\end{array}$ \\
\hline \multicolumn{8}{|c|}{01475840 (site 19) Crum Creek at Whitehorse, Pa. (Lat 3959 54n Long 07527 38w) } \\
\hline $10 / 19 / 95$ & 1028 & 80020 & 4.5 & 204 & 7.5 & 11.0 & 11.5 \\
\hline $10 / 16 / 96$ & 1028 & 80020 & 11 & 206 & 6.8 & 10.5 & 11.5 \\
\hline \multicolumn{8}{|c|}{01476430 (site 20) Ridley Creek at Goshenville, Pa. (Lat 3959 28n Long 07532 40w) } \\
\hline $10 / 25 / 95$ & 1028 & 80020 & 3.5 & 248 & 7.3 & 13.5 & 10.8 \\
\hline $10 / 28 / 96$ & 1028 & 80020 & 7.6 & 265 & 6.8 & 14.5 & 9.5 \\
\hline \multicolumn{8}{|c|}{01476435 (site 21) Ridley Creek at Dutton Mill near West Chester, Pa.(Lat 39 58 52n Long 07531 02w) } \\
\hline $10 / 23 / 95$ & 1028 & 80020 & 10 & 237 & 7.3 & 10.5 & 10.4 \\
\hline $10 / 23 / 96$ & 1028 & 80020 & 22 & 235 & 6.5 & 12.5 & 10.4 \\
\hline \multicolumn{8}{|c|}{01476790 (site 22) East Branch Chester Creek at Green Hill, Pa. (Lat 39 59 49n Long 07535 40w) } \\
\hline $10 / 23 / 95$ & 1028 & 80020 & 1.2 & 384 & 7.0 & 13.5 & 9.8 \\
\hline $10 / 03 / 97$ & 1028 & 80020 & .37 & 371 & 6.8 & 13.5 & 9.5 \\
\hline \multicolumn{8}{|c|}{01476830 (site 23) East Branch Chester Creek at Milltown, Pa. (Lat 3958 21n Long 075 32 57w) } \\
\hline $10 / 24 / 95$ & 1028 & 80020 & 4.7 & 327 & 7.4 & 12.5 & 10.1 \\
\hline $10 / 07 / 96$ & 1028 & 80020 & 12 & 332 & 6.5 & 13.5 & 10.2 \\
\hline \multicolumn{8}{|c|}{01476835 (site 24) East Branch Chester Creek at Westtown, Pa. (Lat 39 56 26n Long 07532 30w) } \\
\hline $10 / 24 / 95$ & 1028 & 80020 & 8.6 & 334 & 7.3 & 13.5 & 9.7 \\
\hline $10 / 07 / 96$ & 1028 & 80020 & 16 & 338 & 6.7 & 12.0 & 9.9 \\
\hline $10 / 06 / 97$ & 1028 & 80020 & 3.2 & 395 & 7.1 & 17.5 & 8.6 \\
\hline \multicolumn{8}{|c|}{01476840 (site 25) Goose Creek trib to E Br Chester Creek nr W Chester, Pa.(Lat 3956 04n Long 07533 31w) } \\
\hline $10 / 25 / 95$ & 1028 & 80020 & 11 & 698 & 7.3 & 15.0 & 8.5 \\
\hline $10 / 04 / 96$ & 1028 & 80020 & 14 & 626 & 7.1 & 13.5 & 10.0 \\
\hline $10 / 03 / 97$ & 1028 & 80020 & 11 & 766 & 7.2 & 15.0 & 9.8 \\
\hline \multicolumn{8}{|c|}{01476848 (site 51) East Br Chester Creek below Goose Creek nr West Chester, Pa. (Lat 3955 45n Long 07532 00w) } \\
\hline $10 / 24 / 95$ & 1028 & 80020 & 24 & 502 & 7.3 & 15.5 & 9.6 \\
\hline $10 / 06 / 97$ & 1028 & 80020 & 12 & 581 & 7.0 & 16.0 & 8.6 \\
\hline \multicolumn{8}{|c|}{01478120 (site 28) East Branch White Clay Creek at Avondale, Pa. (Lat 39 49 42n Long 07546 52w) } \\
\hline $11 / 09 / 95$ & 1028 & 80020 & 8.3 & 347 & 7.4 & 5.5 & 12.3 \\
\hline $11 / 12 / 97$ & 1028 & 80020 & 7.5 & 356 & 7.7 & 7.0 & 10.8 \\
\hline \multicolumn{8}{|c|}{01478190 (site 29) Middle Branch White Clay Creek at Wickerton, Pa. (Lat 39 47 44n Long 075 49 27w) } \\
\hline $11 / 09 / 95$ & 1028 & 80020 & 6.4 & 241 & 7.1 & 5.5 & 12.8 \\
\hline $11 / 12 / 97$ & 1028 & 80020 & 5.5 & 253 & 7.6 & 8.0 & 11.9 \\
\hline \multicolumn{8}{|c|}{01478220 (site 30) West Branch White Clay Creek near Chesterville, Pa. (Lat 3945 56n Long 07547 47w) } \\
\hline $11 / 09 / 95$ & 1028 & 80020 & 5.7 & 194 & 7.0 & 5.5 & 12.5 \\
\hline $11 / 12 / 97$ & 1028 & 80020 & 4.6 & 202 & 7.6 & 8.0 & 12.4 \\
\hline \multicolumn{8}{|c|}{01479680 (site 27) West Branch Red Clay Creek at Kennett Square, Pa. (Lat 3950 13n Long 07543 33w) } \\
\hline $10 / 18 / 95$ & 1028 & 80020 & 4.8 & 361 & 7.5 & 10.5 & 10.9 \\
\hline $11 / 05 / 96$ & 1028 & 80020 & 12 & 301 & 6.7 & 8.0 & 12.1 \\
\hline $10 / 14 / 97$ & 1028 & 80020 & 3.6 & 374 & 7.1 & 15.0 & 8.7 \\
\hline \multicolumn{8}{|c|}{01479800 (site 26) East Branch Red Clay Creek near Five Point, Pa. (Lat 39 49 10n Long 07541 29w) } \\
\hline $10 / 18 / 95$ & 1028 & 80020 & 4.9 & 399 & 7.7 & 11.0 & 11.8 \\
\hline $11 / 05 / 96$ & 1028 & 80020 & 12 & 342 & 6.9 & 8.5 & 13.7 \\
\hline $10 / 14 / 97$ & 1028 & 80020 & 2.7 & 378 & 7.2 & 16.0 & 9.7 \\
\hline \multicolumn{8}{|c|}{01480434 (site 37) West Branch Brandywine Creek at Rock Run, Pa. (Lat 39 59 36n Long 07549 41w) } \\
\hline $10 / 17 / 95$ & 1028 & 80020 & 15 & 252 & 7.4 & 8.5 & 11.0 \\
\hline $10 / 29 / 96$ & 1028 & 80020 & 74 & 212 & 6.5 & 12.0 & 10.8 \\
\hline
\end{tabular}


Table 3. Water-quality data-Continued

\begin{tabular}{|c|c|c|c|c|c|c|}
\hline Date & $\begin{array}{c}\text { Alkalinity } \\
\text { whole water } \\
\text { total, field } \\
\left(\mathrm{mg} / \mathrm{L} \text { as } \mathrm{CaCO}_{3}\right) \\
(00419)\end{array}$ & $\begin{array}{c}\text { Dissolved } \\
\text { chloride } \\
(\mathrm{mg} / \mathrm{L} \text { as Cl) } \\
(00940)\end{array}$ & $\begin{array}{l}\text { Dissolved nitrite } \\
\text { nitrogen } \\
(\mathrm{mg} / \mathrm{L} \text { as } \mathrm{N}) \\
(00613)\end{array}$ & $\begin{array}{c}\text { Dissolved } \\
\mathrm{NO}_{2}+\mathrm{NO}_{3} \\
\text { nitrogen } \\
(\mathrm{mg} / \mathrm{L} \text { as } \mathrm{N}) \\
(00631)\end{array}$ & $\begin{array}{l}\text { Dissolved ammonia } \\
\text { nitrogen } \\
(\mathrm{mg} / \mathrm{L} \text { as } \mathrm{N}) \\
(00608)\end{array}$ & $\begin{array}{c}\text { Dissolved } \\
\text { orthophosphorus } \\
\text { (mg/L as } \mathrm{P}) \\
(00671)\end{array}$ \\
\hline \multicolumn{7}{|c|}{01475840 (site 19) Crum Creek at Whitehorse, Pa. (Lat 3959 54n Long 07527 38w) } \\
\hline $10 / 19 / 95$ & 48 & 16 & 0.010 & 1.40 & $<0.015$ & 0.010 \\
\hline $10 / 16 / 96$ & 50 & 20 & -- & -- & -- & -- \\
\hline \multicolumn{7}{|c|}{01476430 (site 20) Ridley Creek at Goshenville, Pa. (Lat 39 59 28n Long 07532 40w) } \\
\hline $10 / 25 / 95$ & 51 & 25 & .010 & 2.20 & $<.015$ & .040 \\
\hline $10 / 28 / 96$ & 42 & 35 & .020 & 3.20 & $<.015$ & $<.010$ \\
\hline \multicolumn{7}{|c|}{01476435 (site 21) Ridley Creek at Dutton Mill near West Chester, Pa.(Lat 39 58 52n Long 07531 02w) } \\
\hline $10 / 23 / 95$ & 45 & 21 & $<.010$ & 2.40 & .020 & .170 \\
\hline $10 / 23 / 96$ & 53 & 26 & .020 & 3.40 & .030 & .080 \\
\hline \multicolumn{7}{|c|}{01476790 (site 22) East Branch Chester Creek at Green Hill, Pa. (Lat 39 59 49n Long 07535 40w) } \\
\hline $10 / 23 / 95$ & 56 & 55 & $<.010$ & 4.20 & $<.015$ & .020 \\
\hline $10 / 03 / 97$ & 51 & 55 & .016 & 4.32 & $<.015$ & $<.010$ \\
\hline \multicolumn{7}{|c|}{01476830 (site 23) East Branch Chester Creek at Milltown, Pa. (Lat 3958 21n Long 07532 57w) } \\
\hline $10 / 24 / 95$ & 66 & 37 & .040 & 1.80 & .030 & .030 \\
\hline $10 / 07 / 96$ & 57 & 47 & .060 & 1.80 & .130 & $<.010$ \\
\hline \multicolumn{7}{|c|}{01476835 (site 24) East Branch Chester Creek at Westtown, Pa. (Lat 39 56 26n Long 07532 30w) } \\
\hline $10 / 24 / 95$ & 46 & 38 & .030 & 2.40 & .060 & .130 \\
\hline $10 / 07 / 96$ & 55 & 47 & .090 & 2.40 & .220 & .100 \\
\hline $10 / 06 / 97$ & 69 & 50 & .083 & 3.93 & $<.015$ & .272 \\
\hline \multicolumn{7}{|c|}{01476840 (site 25) Goose Creek trib to E Br Chester Creek nr W Chester, Pa.(Lat 3956 04n Long 07533 31w) } \\
\hline $10 / 25 / 95$ & 72 & 86 & .690 & 12.0 & .320 & 2.00 \\
\hline $10 / 04 / 96$ & 45 & 89 & .220 & 14.0 & .330 & 1.10 \\
\hline $10 / 03 / 97$ & 61 & 110 & .108 & 16.9 & .018 & 3.13 \\
\hline \multicolumn{7}{|c|}{01476848 (site 51) East Br Chester Creek below Goose Creek nr West Chester, Pa. (Lat 3955 45n Long 07532 00w) } \\
\hline $10 / 24 / 95$ & 70 & 58 & .370 & 6.80 & .470 & 1.00 \\
\hline $10 / 06 / 97$ & 69 & 78 & .078 & 9.69 & $<.015$ & 1.54 \\
\hline \multicolumn{7}{|c|}{01478120 (site 28) East Branch White Clay Creek at Avondale, Pa. (Lat 39 49 42n Long 075 46 52w) } \\
\hline $11 / 09 / 95$ & 100 & 17 & .010 & 3.90 & $<.015$ & .020 \\
\hline $11 / 12 / 97$ & 85 & 20 & .024 & 4.73 & $<.020$ & .053 \\
\hline \multicolumn{7}{|c|}{01478190 (site 29) Middle Branch White Clay Creek at Wickerton, Pa. (Lat 39 47 44n Long 075 49 27w) } \\
\hline $11 / 09 / 95$ & 43 & 16 & .030 & 4.90 & $<.015$ & .170 \\
\hline $11 / 12 / 97$ & 41 & 20 & .026 & 4.97 & $<.020$ & .230 \\
\hline \multicolumn{7}{|c|}{01478220 (site 30) West Branch White Clay Creek near Chesterville, Pa. (Lat 39 45 56n Long 075 47 47w) } \\
\hline $11 / 09 / 95$ & 30 & 15 & .020 & 2.80 & $<.015$ & $<.010$ \\
\hline $11 / 12 / 97$ & 26 & 17 & .028 & 3.27 & $<.020$ & .039 \\
\hline \multicolumn{7}{|c|}{01479680 (site 27) West Branch Red Clay Creek at Kennett Square, Pa. (Lat 39 50 13n Long 075 43 33w) } \\
\hline $10 / 18 / 95$ & 89 & 18 & .010 & 4.80 & .040 & .050 \\
\hline $11 / 05 / 96$ & 75 & 18 & .020 & 5.40 & .020 & $<.010$ \\
\hline $10 / 14 / 97$ & 97 & 26 & .023 & 4.77 & .039 & .021 \\
\hline \multicolumn{7}{|c|}{01479800 (site 26) East Branch Red Clay Creek near Five Point, Pa. (Lat 39 49 10n Long 07541 29w) } \\
\hline $10 / 18 / 95$ & 68 & 26 & $<.010$ & 3.70 & $<.015$ & .060 \\
\hline $11 / 05 / 96$ & 72 & 27 & .040 & 4.50 & .020 & .020 \\
\hline $10 / 14 / 97$ & 123 & 29 & .019 & 2.92 & .026 & .066 \\
\hline \multicolumn{7}{|c|}{01480434 (site 37) West Branch Brandywine Creek at Rock Run, Pa. (Lat 39 59 36n Long 07549 41w) } \\
\hline $10 / 17 / 95$ & 47 & 16 & $<.010$ & 2.80 & $<.015$ & .190 \\
\hline $10 / 29 / 96$ & 43 & 15 & .020 & 3.60 & $<.015$ & .020 \\
\hline
\end{tabular}


Table 3. Water-quality data-Continued

$[\mu \mathrm{S} / \mathrm{cm}$, microsiemens per centimeter at 25 degrees Celsius; mg/L, milligrams per liter, <, less than; --, no data]

\begin{tabular}{|c|c|c|c|c|c|c|c|}
\hline Date & $\begin{array}{c}\text { Agency } \\
\text { collecting } \\
\text { sample } \\
\text { (code number) } \\
(00027)\end{array}$ & $\begin{array}{c}\text { Agency } \\
\text { analyzing } \\
\text { sample } \\
\text { (code number) } \\
(00028)\end{array}$ & $\begin{array}{l}\text { Instantaneous } \\
\text { discharge } \\
\text { (cubic feet per } \\
\text { second) } \\
(00061)\end{array}$ & $\begin{array}{c}\text { Specific } \\
\text { conductance } \\
(\mu \mathrm{S} / \mathrm{cm}) \\
(00095)\end{array}$ & $\begin{array}{c}\mathrm{pH}, \text { water, } \\
\text { whole field } \\
\text { (standard units) } \\
(00400)\end{array}$ & $\begin{array}{c}\text { Water } \\
\text { temperature } \\
\text { (degrees } \\
\text { Celsius) } \\
(00010)\end{array}$ & $\begin{array}{c}\text { Dissolved } \\
\text { oxygen } \\
(\mathrm{mg} / \mathrm{L}) \\
(00300)\end{array}$ \\
\hline \multicolumn{8}{|c|}{01480629 (site 46) Buck Run at Doe Run, Pa. (Lat 3955 46n Long 075 49 24w) } \\
\hline $11 / 28 / 95$ & 1028 & 80020 & 21 & 240 & 7.7 & 8.5 & 12.9 \\
\hline $11 / 06 / 97$ & 1028 & 80020 & 8.8 & 238 & 7.7 & 7.5 & 12.3 \\
\hline \multicolumn{8}{|c|}{01480632 (site 45) Doe Run at Springdell, Pa. (Lat 39 54 25n Long 07549 42w) } \\
\hline $11 / 28 / 95$ & 1028 & 80020 & 10 & 156 & 7.2 & 8.5 & 12.1 \\
\hline $11 / 06 / 97$ & 1028 & 80020 & 5.9 & 153 & 7.3 & 6.5 & 12.2 \\
\hline \multicolumn{8}{|c|}{01480640 (site 38) West Branch Brandywine Creek at Wawaset, Pa. (Lat 39 55 34n Long 07539 47w) } \\
\hline $11 / 20 / 95$ & 1028 & 80020 & 181 & 253 & 7.2 & 6.5 & 12.1 \\
\hline $10 / 15 / 96$ & 1028 & 80020 & 168 & 238 & 6.4 & 12.0 & 10.3 \\
\hline $10 / 10 / 97$ & 1028 & 80020 & 43 & 285 & 7.0 & 18.0 & 8.2 \\
\hline \multicolumn{8}{|c|}{01480648 (site 48) East Branch Brandywine Creek near Cupola, Pa. (Lat 4005 41n Long 07551 14w) } \\
\hline $11 / 27 / 95$ & 1028 & 80020 & 5.4 & 207 & 7.0 & 4.5 & 12.7 \\
\hline \multicolumn{8}{|c|}{01480653 (site 42) East Branch Brandywine Creek at Glenmoore, Pa. (Lat 4005 48n Long 075 46 44w) } \\
\hline $12 / 01 / 95$ & 1028 & 80020 & 13 & 196 & 7.3 & 4.0 & 13.1 \\
\hline \multicolumn{8}{|c|}{01480656 (site 47) Indian Run near Springton, Pa. (Lat 4004 33n Long 07546 52w) } \\
\hline $11 / 27 / 95$ & 1028 & 80020 & 2.5 & 182 & 7.2 & 6.5 & 13.0 \\
\hline \multicolumn{8}{|c|}{01480700 (site 36) East Branch Brandywine Creek near Downingtown, Pa. (Lat 40 02 05n Long 075 42 32w) } \\
\hline $10 / 17 / 95$ & 1028 & 80020 & 19 & 222 & 7.5 & 11.0 & 11.4 \\
\hline $10 / 29 / 96$ & 1028 & 80020 & 85 & 191 & 6.4 & 11.0 & 10.5 \\
\hline \multicolumn{8}{|c|}{01480903 (site 44) Valley Creek at Mullsteins Meadows nr Downingtown, Pa. (Lat 39 58 31n Long 07539 48w) } \\
\hline $11 / 21 / 95$ & 1028 & 80020 & 21 & 415 & 7.9 & 8.5 & 13.2 \\
\hline $11 / 04 / 97$ & 1028 & 80020 & 7.2 & 421 & 8.0 & 9.5 & 11.0 \\
\hline \multicolumn{8}{|c|}{01480950 (site 39) East Branch Brandywine Creek at Wawaset, Pa. (Lat 39 55 35n Long 07538 54w) } \\
\hline $11 / 20 / 95$ & 1028 & 80020 & 138 & 271 & 7.3 & 8.0 & 12.3 \\
\hline $10 / 15 / 96$ & 1028 & 80020 & 144 & 293 & 6.6 & 12.5 & 11.2 \\
\hline $10 / 29 / 97$ & 1028 & 80020 & 50 & 350 & 7.3 & 8.0 & 11.2 \\
\hline \multicolumn{8}{|c|}{01481030 (site 40) Brandywine Creek near Chadds Ford, Pa. (Lat 3951 15n Long 075 35 58w) } \\
\hline $10 / 13 / 95$ & 1028 & 80020 & 159 & 345 & 7.6 & 16.0 & 9.2 \\
\hline $11 / 13 / 97$ & 1028 & 80020 & 125 & 299 & 7.4 & 6.5 & 11.1 \\
\hline \multicolumn{8}{|c|}{01494900 (site 31) East Branch Big Elk Creek at Elkview, Pa. (Lat 3948 48n Long 07554 04w) } \\
\hline $11 / 30 / 95$ & 1028 & 80020 & 8.7 & 180 & 7.4 & 3.5 & 14.3 \\
\hline $10 / 25 / 96$ & 1028 & 80020 & 15 & 172 & 6.0 & 11.0 & 11.0 \\
\hline $10 / 31 / 97$ & 1028 & 80020 & 3.6 & 184 & 6.7 & 9.5 & 12.0 \\
\hline \multicolumn{8}{|c|}{01494950 (site 32) West Branch Big Elk Creek near Oxford, Pa. (Lat 3946 47n Long 07555 27w) } \\
\hline $11 / 30 / 95$ & 1028 & 80020 & 8.2 & 237 & 7.0 & 4.5 & 14.9 \\
\hline $10 / 25 / 96$ & 1028 & 80020 & 14 & 201 & 6.2 & 11.0 & 11.4 \\
\hline \multicolumn{8}{|c|}{01578340 (site 33) East Branch Octoraro Creek at Christiana, Pa. (Lat 39 56 57n Long 075 59 38w) } \\
\hline $10 / 26 / 95$ & 1028 & 80020 & 6.8 & 321 & 7.3 & 9.0 & 12.0 \\
\hline $10 / 30 / 96$ & 1028 & 80020 & 15 & 304 & 6.7 & 12.0 & 11.5 \\
\hline $10 / 31 / 97$ & 1028 & 80020 & 4.5 & 320 & 6.7 & 7.0 & 10.6 \\
\hline \multicolumn{8}{|c|}{01578343 (site 34) Valley Creek near Atglen, Pa. (Lat 39 56 23n Long 07559 06w) } \\
\hline $10 / 26 / 95$ & 1028 & 80020 & 5.3 & 341 & 7.4 & 10.5 & 10.9 \\
\hline $10 / 30 / 96$ & 1028 & 80020 & 15 & 298 & 6.4 & 11.0 & 9.9 \\
\hline
\end{tabular}


Table 3. Water-quality data-Continued

\begin{tabular}{|c|c|c|c|c|c|c|}
\hline Date & $\begin{array}{c}\text { Alkalinity } \\
\text { whole water } \\
\text { total, field } \\
\left(\mathrm{mg} / \mathrm{L} \text { as } \mathrm{CaCO}_{3}\right) \\
(00419)\end{array}$ & $\begin{array}{l}\text { Dissolved } \\
\text { chloride } \\
(\mathrm{mg} / \mathrm{L} \text { as } \mathrm{Cl}) \\
(00940)\end{array}$ & $\begin{array}{l}\text { Dissolved nitrite } \\
\text { nitrogen } \\
(\mathrm{mg} / \mathrm{L} \text { as } \mathrm{N}) \\
(00613)\end{array}$ & $\begin{array}{c}\text { Dissolved } \\
\mathrm{NO}_{2}+\mathrm{NO}_{3} \\
\text { nitrogen } \\
(\mathrm{mg} / \mathrm{L} \text { as } \mathrm{N}) \\
(00631)\end{array}$ & $\begin{array}{c}\text { Dissolved ammonia } \\
\text { nitrogen } \\
(\mathrm{mg} / \mathrm{L} \text { as } \mathrm{N}) \\
(00608)\end{array}$ & $\begin{array}{c}\text { Dissolved } \\
\text { orthophosphorus } \\
\text { (mg/L as } \mathrm{P}) \\
(00671)\end{array}$ \\
\hline \multicolumn{7}{|c|}{01480629 (site 46) Buck Run at Doe Run, Pa. (Lat 3955 46n Long 07549 24w) } \\
\hline $11 / 28 / 95$ & 42 & 18 & 0.020 & 5.50 & $<0.015$ & 0.020 \\
\hline $11 / 06 / 97$ & 47 & 17 & .024 & 4.26 & $<.020$ & .026 \\
\hline \multicolumn{7}{|c|}{01480632 (site 45) Doe Run at Springdell, Pa. (Lat 39 54 25n Long 07549 42w) } \\
\hline $11 / 28 / 95$ & 24 & 11 & $<.010$ & 5.00 & $<.015$ & $<.010$ \\
\hline $11 / 06 / 97$ & 23 & 11 & .030 & 4.81 & $<.020$ & .026 \\
\hline \multicolumn{7}{|c|}{01480640 (site 38) West Branch Brandywine Creek at Wawaset, Pa. (Lat 3955 34n Long 075 39 47w) } \\
\hline $11 / 20 / 95$ & 46 & 18 & .010 & 3.70 & $<.015$ & .040 \\
\hline $10 / 15 / 96$ & 70 & 18 & .020 & 3.90 & .040 & .040 \\
\hline $10 / 10 / 97$ & 67 & 24 & .053 & 2.80 & $<.015$ & $<.010$ \\
\hline \multicolumn{7}{|c|}{01480648 (site 48) East Branch Brandywine Creek near Cupola, Pa. (Lat 4005 41n Long 07551 14w) } \\
\hline $11 / 27 / 95$ & 57 & 13 & .020 & 3.30 & .110 & .010 \\
\hline \multicolumn{7}{|c|}{01480653 (site 42) East Branch Brandywine Creek at Glenmoore, Pa. (Lat 4005 48n Long 075 46 44w) } \\
\hline $12 / 01 / 95$ & 49 & 13 & .010 & 3.70 & .070 & .010 \\
\hline \multicolumn{7}{|c|}{01480656 (site 47) Indian Run near Springton, Pa. (Lat 4004 33n Long 075 46 52w) } \\
\hline $11 / 27 / 95$ & 46 & 11 & .010 & 3.80 & $<.015$ & .060 \\
\hline \multicolumn{7}{|c|}{01480700 (site 36) East Branch Brandywine Creek near Downingtown, Pa. (Lat 4002 05n Long 07542 32w) } \\
\hline $10 / 17 / 95$ & 47 & 17 & $<.010$ & 1.40 & .020 & .030 \\
\hline $10 / 29 / 96$ & 44 & 17 & .010 & 2.20 & $<.020$ & .020 \\
\hline \multicolumn{7}{|c|}{01480903 (site 44) Valley Creek at Mullsteins Meadows nr Downingtown, Pa. (Lat 39 58 31n Long 07539 48w) } \\
\hline $11 / 21 / 95$ & 118 & 29 & .010 & 2.10 & $<.015$ & $<.010$ \\
\hline $11 / 04 / 97$ & 119 & 29 & .031 & 2.23 & $<.020$ & .027 \\
\hline \multicolumn{7}{|c|}{01480950 (site 39) East Branch Brandywine Creek at Wawaset, Pa. (Lat 3955 35n Long 075 38 54w) } \\
\hline $11 / 20 / 95$ & 60 & 22 & .020 & 2.10 & .090 & .090 \\
\hline $10 / 15 / 96$ & 95 & 27 & .020 & 2.90 & .050 & .050 \\
\hline $10 / 29 / 97$ & 63 & 33 & .015 & 3.41 & $<.015$ & .168 \\
\hline \multicolumn{7}{|c|}{01481030 (site 40) Brandywine Creek near Chadds Ford, Pa. (Lat 3951 15n Long 075 35 58w) } \\
\hline $10 / 13 / 95$ & 77 & 31 & $<.010$ & 3.00 & $<.015$ & .100 \\
\hline $11 / 13 / 97$ & 68 & 26 & .025 & 2.91 & $<.020$ & .169 \\
\hline \multicolumn{7}{|c|}{01494900 (site 31) East Branch Big Elk Creek at Elkview, Pa. (Lat 3948 48n Long 07554 04w) } \\
\hline $11 / 30 / 95$ & 24 & 16 & $<.010$ & 5.10 & $<.015$ & .080 \\
\hline $10 / 25 / 96$ & 31 & 15 & .010 & 4.00 & $<.015$ & .020 \\
\hline $10 / 31 / 97$ & 39 & 16 & $<.010$ & 6.30 & $<.020$ & .094 \\
\hline \multicolumn{7}{|c|}{01494950 (site 32) West Branch Big Elk Creek near Oxford, Pa. (Lat 3946 47n Long 07555 27w) } \\
\hline $11 / 30 / 95$ & 32 & 28 & .010 & 5.30 & $<.015$ & .030 \\
\hline $10 / 25 / 96$ & 28 & 21 & .010 & 5.80 & $<.015$ & .020 \\
\hline \multicolumn{7}{|c|}{01578340 (site 33) East Branch Octoraro Creek at Christiana, Pa. (Lat 39 56 57n Long 075 59 38w) } \\
\hline $10 / 26 / 95$ & 50 & 19 & .020 & 8.70 & $<.015$ & .040 \\
\hline $10 / 30 / 96$ & 46 & 19 & .020 & 10.0 & $<.015$ & .010 \\
\hline $10 / 31 / 97$ & 83 & 21 & .045 & 8.98 & $<.020$ & .024 \\
\hline \multicolumn{7}{|c|}{01578343 (site 34) Valley Creek near Atglen, Pa. (Lat 3956 23n Long 07559 06w) } \\
\hline $10 / 26 / 95$ & 62 & 21 & .040 & 7.10 & .280 & .080 \\
\hline $10 / 30 / 96$ & 53 & 19 & .030 & 8.70 & .160 & .030 \\
\hline
\end{tabular}




\section{Table 4. Results of stream-bottom sediment sampling}

$[\mu \mathrm{g} / \mathrm{g}$; microgram per gram; g/kg, gram per kilogram; $\mu \mathrm{g} / \mathrm{kg}$, microgram per kilogram; PCB, Polychlorinated biphenyls; PCN, Polychlorinated naphthalenes; <, less than; E, estimated; --, no data]

\begin{tabular}{|c|c|c|c|c|c|c|c|c|c|}
\hline Date & $\begin{array}{c}\text { Arsenic, } \\
\text { total in bottom } \\
\text { material } \\
(\mu \mathrm{g} / \mathrm{g} \text { as As })\end{array}$ & $\begin{array}{l}\text { Cadmium, } \\
\text { recoverable } \\
\text { from bottom } \\
\text { material } \\
(\mu \mathrm{g} / \mathrm{g} \text { as } \mathrm{Cd})\end{array}$ & $\begin{array}{c}\text { Chromium, } \\
\text { recoverable } \\
\text { from bottom } \\
\text { material } \\
(\mu \mathrm{g} / \mathrm{g} \text { as } \mathrm{Cr})\end{array}$ & $\begin{array}{c}\text { Copper, } \\
\text { recoverable } \\
\text { from bottom } \\
\text { material } \\
(\mu \mathrm{g} / \mathrm{g} \text { as } \mathrm{Cu})\end{array}$ & $\begin{array}{c}\text { Iron, } \\
\text { recoverable } \\
\text { from bottom } \\
\text { material } \\
(\mu \mathrm{g} / \mathrm{g} \text { as } \mathrm{Fe})\end{array}$ & $\begin{array}{c}\text { Lead, } \\
\text { recoverable } \\
\text { from bottom } \\
\text { material } \\
(\mu \mathrm{g} / \mathrm{g} \text { as } \mathrm{Pb})\end{array}$ & $\begin{array}{c}\text { Manganese, } \\
\text { recoverable } \\
\text { from bottom } \\
\text { material } \\
(\mu \mathrm{g} / \mathrm{g} \text { as } \mathrm{Mn})\end{array}$ & $\begin{array}{c}\text { Mercury, } \\
\text { recoverable } \\
\text { from bottom } \\
\text { material } \\
(\mu \mathrm{g} / \mathrm{g} \text { as } \mathrm{Hg})\end{array}$ & $\begin{array}{c}\text { Zinc, } \\
\text { recoverable } \\
\text { from bottom } \\
\text { material } \\
(\mu \mathrm{g} / \mathrm{g} \text { as } \mathrm{Zn})\end{array}$ \\
\hline \multicolumn{10}{|c|}{01472080 (site10) Pigeon Creek near Parker Ford, Pa. (Lat 4012 03n Long 075 37 10w) } \\
\hline $11 / 06 / 96$ & 2 & $<1$ & 1 & 0 & 1,700 & $<10$ & 53 & $<0.01$ & 7 \\
\hline \multicolumn{10}{|c|}{01472109 (site 6) Stony Run near Spring City, Pa. (Lat 4010 11n Long 07534 45w) } \\
\hline $11 / 06 / 96$ & 3 & $<1$ & 7 & 10 & 7,400 & $<10$ & 220 & .02 & 30 \\
\hline \multicolumn{10}{|c|}{01472138 (site 13) French Creek near Coventryville, Pa. (Lat 4010 14n Long 075 41 50w) } \\
\hline $10 / 01 / 97$ & -- & $<1$ & 42 & 29 & 22,000 & 30 & 440 & .04 & 100 \\
\hline \multicolumn{10}{|c|}{01472140 (site 12) South Branch French Creek at Coventryville, Pa. (Lat 4009 18n Long 075 42 52w) } \\
\hline $10 / 09 / 97$ & 2 & $<1$ & 18 & 26 & 23,000 & 20 & 350 & .02 & 60 \\
\hline \multicolumn{10}{|c|}{01472154 (site 14) French Creek near Pughtown, Pa. (Lat 4009 17n Long 07538 25w) } \\
\hline $10 / 08 / 97$ & 3 & $<1$ & 32 & 27 & 28,000 & 40 & 860 & .04 & 110 \\
\hline \multicolumn{10}{|c|}{01472170 (site 1) Pickering Creek near Eagle, Pa. (Lat 4004 43n Long 07539 14w) } \\
\hline $10 / 31 / 96$ & 3 & $<1$ & 50 & 20 & 27,000 & 20 & 350 & .01 & 90 \\
\hline \multicolumn{10}{|c|}{014721854 (site 3) Pickering Creek at Merlin, Pa. (Lat 4006 25n Long 075 35 34w) } \\
\hline $10 / 16 / 97$ & 2 & $<1$ & 30 & 16 & 22,000 & 20 & 240 & .04 & 100 \\
\hline \multicolumn{10}{|c|}{014721884 (site 4) Pickering Creek at Charlestown Rd at Charlestown, Pa. (Lat 40 05 57n Long 07533 20w) } \\
\hline $10 / 02 / 96$ & 2 & $<1$ & 20 & 12 & 16,000 & 20 & 220 & .03 & 70 \\
\hline \multicolumn{10}{|c|}{01475300 (site 17) Darby Creek at Waterloo Mills near Devon, Pa. (Lat 4001 21n Long 075 25 20w) } \\
\hline $10 / 16 / 96$ & 2 & $<1$ & 30 & 12 & 15,000 & 20 & 170 & .02 & 60 \\
\hline \multicolumn{10}{|c|}{01475840 (site 19) Crum Creek at Whitehorse, Pa. (Lat 39 59 54n Long 07527 38w) } \\
\hline $10 / 16 / 96$ & 3 & $<1$ & 20 & 10 & 4,400 & 20 & 230 & .04 & 40 \\
\hline \multicolumn{10}{|c|}{01476790 (site 22) East Branch Chester Creek at Green Hill, Pa. (Lat 3959 49n Long 075 35 40w) } \\
\hline $10 / 09 / 97$ & 4 & $<1$ & 29 & 31 & 40,000 & 40 & 1100 & .04 & 130 \\
\hline \multicolumn{10}{|c|}{01476830 (site 23) East Branch Chester Creek at Milltown, Pa. (Lat 3958 21n Long 075 32 57w) } \\
\hline $10 / 07 / 96$ & 2 & $<1$ & 20 & 10 & 17,000 & 20 & 440 & .02 & 50 \\
\hline \multicolumn{10}{|c|}{01480629 (site 46) Buck Run at Doe Run, Pa. (Lat 3955 46n Long 07549 24w) } \\
\hline $11 / 28 / 95$ & 3 & $<1$ & 6 & 11 & 1,800 & 20 & 350 & .26 & 40 \\
\hline \multicolumn{10}{|c|}{01480632 (site 45) Doe Run at Springdell, Pa. (Lat 39 54 25n Long 07549 42w) } \\
\hline $11 / 28 / 95$ & 2 & $<1$ & 5 & 10 & 1,600 & 10 & 250 & .02 & 30 \\
\hline \multicolumn{10}{|c|}{01480648 (site 48) East Branch Brandywine Creek near Cupola, Pa. (lat 4005 41n long 07551 14w) } \\
\hline $11 / 27 / 95$ & 1 & $<1$ & 2 & 0 & 1,400 & $<10$ & 220 & .02 & 20 \\
\hline \multicolumn{10}{|c|}{01480653 (site 42) East Branch Brandywine Creek at Glenmoore, Pa. (Lat 4005 48n Long 07546 44w) } \\
\hline $12 / 01 / 95$ & 2 & $<1$ & 3 & 0 & 2,700 & $<10$ & 110 & .02 & 30 \\
\hline \multicolumn{10}{|c|}{01480656 (site 47) Indian Run near Springton, Pa. (Lat 4004 33n Long 075 46 52w) } \\
\hline $11 / 27 / 95$ & 2 & $<1$ & 4 & 0 & 1,600 & $<10$ & 120 & .02 & 20 \\
\hline \multicolumn{10}{|c|}{01480903 (site 44) Valley Creek at Mullsteins Meadows near Downingtown, Pa. (Lat 3958 31n Long 07539 48w) } \\
\hline $11 / 21 / 95$ & 2 & $<1$ & 10 & 14 & 1,400 & 20 & 260 & .03 & 80 \\
\hline \multicolumn{10}{|c|}{01481030 (site 40) Brandywine Creek near Chadds Ford, Pa. (Lat 3951 15n Long 075 35 58w) } \\
\hline $11 / 13 / 97$ & 3 & $<1$ & 34 & 27 & 25,000 & 70 & 720 & $<.10$ & 120 \\
\hline
\end{tabular}


Table 4. Results of stream-bottom sediment sampling—Continued

\begin{tabular}{|c|c|c|c|c|c|c|c|c|}
\hline Date & $\begin{array}{c}\text { Carbon, } \\
\text { total inorganic } \\
\text { and organic in } \\
\text { bottom material } \\
\text { (g/kg as C) }\end{array}$ & $\begin{array}{c}\text { Aldrin, } \\
\text { total in bottom } \\
\text { material } \\
(\mu \mathrm{g} / \mathrm{kg})\end{array}$ & $\begin{array}{c}\text { Chlordane, } \\
\text { total in bottom } \\
\text { material } \\
(\mu \mathrm{g} / \mathrm{kg})\end{array}$ & $\begin{array}{c}\text { p,p'-DDD, } \\
\text { recoverable } \\
\text { in bottom } \\
\text { material } \\
(\mu \mathrm{g} / \mathrm{kg})\end{array}$ & $\begin{array}{c}\text { p,p'-DDE, } \\
\text { recoverable } \\
\text { in bottom } \\
\text { material } \\
(\mu \mathrm{g} / \mathrm{kg})\end{array}$ & $\begin{array}{c}\text { p,p'-DDT, } \\
\text { recoverable } \\
\text { in bottom } \\
\text { material } \\
(\mu \mathrm{g} / \mathrm{kg})\end{array}$ & $\begin{array}{c}\text { Dieldrin, } \\
\text { total in bottom } \\
\text { material } \\
(\mu \mathrm{g} / \mathrm{kg})\end{array}$ & $\begin{array}{l}\text { Endosulfan, } \\
\text { total in bottom } \\
\text { material } \\
(\mu \mathrm{g} / \mathrm{kg})\end{array}$ \\
\hline \multicolumn{9}{|c|}{01472080 (site 10) Pigeon Creek near Parker Ford, Pa. (Lat 4012 03n Long 07537 10w) } \\
\hline $11 / 06 / 96$ & 5.5 & $<0.100$ & $<1.00$ & $<0.100$ & $<0.100$ & $<0.100$ & $<0.100$ & $<0.100$ \\
\hline \multicolumn{9}{|c|}{01472109 (site 6) Stony Run near Spring City, Pa. (Lat 4010 11n Long 07534 45w) } \\
\hline $11 / 06 / 96$ & 4.1 & $<.100$ & 10.0 & E1.30 & $<1.00$ & $<1.00$ & 1.60 & $<2.00$ \\
\hline \multicolumn{9}{|c|}{01472138 (site 13) French Creek near Coventryville, Pa. (Lat 4010 14n Long 075 41 50w) } \\
\hline $10 / 01 / 97$ & 44 & $<.200$ & 12.0 & $<.500$ & 1.10 & E. 540 & .580 & .200 \\
\hline \multicolumn{9}{|c|}{01472140 (site 12) South Branch French Creek at Coventryville, Pa. (Lat 4009 18n Long 075 42 52w) } \\
\hline $10 / 09 / 97$ & 8.9 & $<.200$ & $<3.00$ & $<.500$ & .260 & $<.500$ & $<.200$ & $<.200$ \\
\hline \multicolumn{9}{|c|}{01472154 (site 14) French Creek near Pughtown, Pa. (Lat 4009 17n Long 07538 25w) } \\
\hline $10 / 08 / 97$ & 16 & $<.200$ & $<3.00$ & $<.500$ & .430 & $<.500$ & .240 & $<.200$ \\
\hline \multicolumn{9}{|c|}{01472170 (site 1) Pickering Creek near Eagle, Pa. (Lat 4004 43n Long 07539 14w) } \\
\hline $10 / 31 / 96$ & 28 & .100 & 2.00 & .200 & .300 & .100 & $<.400$ & $<.100$ \\
\hline \multicolumn{9}{|c|}{014721854 (site 3) Pickering Creek at Merlin, Pa. (Lat 4006 25n Long 075 35 34w) } \\
\hline $10 / 16 / 97$ & 16 & $<.200$ & $<3.00$ & $<.500$ & .600 & $<.500$ & .300 & $<.200$ \\
\hline \multicolumn{9}{|c|}{014721884 (site 4) Pickering Creek at Charlestown Rd at Charlestown, Pa. (Lat 4005 57n Long 07533 20w) } \\
\hline $10 / 02 / 96$ & 22 & $<.100$ & 2.00 & E.300 & .400 & E.200 & .300 & $<.100$ \\
\hline \multicolumn{9}{|c|}{01475300 (site 17) Darby Creek at Waterloo Mills near Devon, Pa. (Lat 4001 21n Long 07525 20w) } \\
\hline $10 / 16 / 96$ & 11 & .200 & 14.0 & $\mathrm{E} 1.70$ & 1.40 & E1.40 & 2.00 & $<.100$ \\
\hline \multicolumn{9}{|c|}{01475840 (site 19) Crum Creek at Whitehorse, Pa. (Lat 39 59 54n Long 07527 38w) } \\
\hline $10 / 16 / 96$ & 10 & $<.100$ & 6.00 & E.900 & .700 & E.600 & .300 & $<.100$ \\
\hline \multicolumn{9}{|c|}{01476790 (site 22) East Branch Chester Creek at Green Hill, Pa. (Lat 3959 49n Long 07535 40w) } \\
\hline $10 / 09 / 97$ & 32 & $<.300$ & 32.0 & 1.10 & 3.10 & E1.70 & 3.90 & $<.200$ \\
\hline \multicolumn{9}{|c|}{01476830 (site 23) East Branch Chester Creek at Milltown, Pa. (Lat 3958 21n Long 07532 57w) } \\
\hline $10 / 07 / 96$ & 6.6 & $<.100$ & 7.00 & E.400 & .300 & E.200 & .300 & $<.100$ \\
\hline \multicolumn{9}{|c|}{01480629 (site 46) Buck Run at Doe Run, Pa. (Lat 3955 46n Long 07549 24w) } \\
\hline $11 / 28 / 95$ & 14 & $<.100$ & 9.00 & 1.00 & .700 & .300 & .200 & $<.100$ \\
\hline \multicolumn{9}{|c|}{01480632 (site 45) Doe Run at Springdell, Pa. (Lat 3954 25n Long 07549 42w) } \\
\hline $11 / 28 / 95$ & 20 & $<.100$ & 1.00 & .300 & .800 & .200 & $<.100$ & $<.100$ \\
\hline \multicolumn{9}{|c|}{01480648 (site 48) East Branch Brandywine Creek near Cupola, Pa. (Lat 4005 41n long 07551 14w) } \\
\hline $11 / 27 / 95$ & 19 & $<.100$ & 1.00 & .400 & .700 & .200 & .200 & $<.100$ \\
\hline \multicolumn{9}{|c|}{01480653 (site 42) East Branch Brandywine Creek at Glenmoore, Pa. (Lat 4005 48n Long 075 46 44w) } \\
\hline $12 / 01 / 95$ & 5.9 & $<.100$ & $<1.00$ & $<.100$ & $<.100$ & $<.100$ & $<.100$ & $<.100$ \\
\hline \multicolumn{9}{|c|}{01480656 (site 47) Indian Run near Springton, Pa. (Lat 4004 33n Long 07546 52w) } \\
\hline $11 / 27 / 95$ & 27 & $<.100$ & $<1.00$ & .200 & .400 & $<.100$ & .100 & $<.100$ \\
\hline \multicolumn{9}{|c|}{01480903 (site 44) Valley Creek at Mullsteins Meadows near Downingtown, Pa. (Lat 3958 31n Long 07539 48w) } \\
\hline $11 / 21 / 95$ & 21 & $<.100$ & 5.00 & .300 & .900 & .400 & .600 & $<.100$ \\
\hline \multicolumn{9}{|c|}{01481030 (site 40) Brandywine Creek near Chadds Ford, Pa. (Lat 3951 15n Long 07535 58w) } \\
\hline $11 / 13 / 97$ & 20 & $<.200$ & 5.40 & .600 & 1.30 & E1.10 & .820 & $<.200$ \\
\hline
\end{tabular}


Table 4. Results of stream-bottom sediment sampling-Continued

\begin{tabular}{|c|c|c|c|c|c|c|c|c|c|c|}
\hline Date & $\begin{array}{l}\text { Endrin, } \\
\text { total in } \\
\text { bottom } \\
\text { material } \\
(\mu \mathrm{g} / \mathrm{kg})\end{array}$ & $\begin{array}{l}\text { Heptachlor, } \\
\text { total in } \\
\text { bottom } \\
\text { material } \\
(\mu \mathrm{g} / \mathrm{kg})\end{array}$ & $\begin{array}{l}\text { Heptachlor } \\
\text { epoxide, } \\
\text { total in } \\
\text { bottom } \\
\text { material } \\
(\mu \mathrm{g} / \mathrm{kg})\end{array}$ & $\begin{array}{l}\text { Lindane, } \\
\text { total in } \\
\text { bottom } \\
\text { material } \\
(\mu \mathrm{g} / \mathrm{kg})\end{array}$ & $\begin{array}{c}\text { Methoxychlor, } \\
\text { total in bottom } \\
\text { material } \\
(\mu \mathrm{g} / \mathrm{kg})\end{array}$ & $\begin{array}{c}\text { Mirex, total } \\
\text { in bottom } \\
\text { material } \\
(\mu \mathrm{g} / \mathrm{kg})\end{array}$ & $\begin{array}{c}\text { Perthane, } \\
\text { in bottom } \\
\text { material } \\
(\mu \mathrm{g} / \mathrm{kg})\end{array}$ & $\begin{array}{c}\text { Toxaphene, } \\
\text { total in } \\
\text { bottom } \\
\text { material } \\
(\mu \mathrm{g} / \mathrm{kg})\end{array}$ & $\begin{array}{l}\text { PCB, } \\
\text { total in } \\
\text { bottom } \\
\text { material } \\
(\mu \mathrm{g} / \mathrm{kg})\end{array}$ & $\begin{array}{l}\mathrm{PCN}, \\
\text { total in } \\
\text { bottom } \\
\text { material } \\
(\mu \mathrm{g} / \mathrm{kg})\end{array}$ \\
\hline \multicolumn{11}{|c|}{01472080 (site 10) Pigeon Creek near Parker Ford, Pa. (Lat 4012 03n Long 075 37 10w) } \\
\hline $11 / 06 / 96$ & $<0.100$ & $<0.100$ & $<0.100$ & $<0.100$ & $<0.800$ & $<0.100$ & $<1.00$ & $<10.0$ & $<1.00$ & $<1.00$ \\
\hline \multicolumn{11}{|c|}{01472109 (site 6) Stony Run near Spring City, Pa. (Lat 4010 11n Long 075 34 45w) } \\
\hline $11 / 06 / 96$ & $<1.00$ & $<.100$ & $<1.00$ & $<1.00$ & $<1.00$ & $<.100$ & $<10.0$ & $<100$ & 2.00 & $<1.00$ \\
\hline \multicolumn{11}{|c|}{01472138 (site 13) French Creek near Coventryville, Pa. (Lat 4010 14n Long 075 41 50w) } \\
\hline $10 / 01 / 97$ & $<.200$ & $<.200$ & $<.200$ & $<.200$ & $<2.50$ & $<.200$ & -- & $<50.0$ & 10.0 & -- \\
\hline \multicolumn{11}{|c|}{01472140 (site 12) South Branch French Creek at Coventryville, Pa. (Lat 4009 18n Long 07542 52w) } \\
\hline $10 / 09 / 97$ & $<.200$ & $<.200$ & $<.200$ & $<.200$ & $<2.50$ & $<.200$ & -- & $<50.0$ & $<5.00$ & -- \\
\hline \multicolumn{11}{|c|}{01472154 (site 14) French Creek near Pughtown, Pa. (Lat 4009 17n Long 07538 25w) } \\
\hline $10 / 08 / 97$ & $<.200$ & $<.200$ & $<.200$ & $<.200$ & $<2.50$ & $<.200$ & -- & $<50.0$ & $<5.00$ & -- \\
\hline \multicolumn{11}{|c|}{01472170 (site 1) Pickering Creek near Eagle, Pa. (Lat 4004 43n Long 07539 14w) } \\
\hline $10 / 31 / 96$ & $<.100$ & $<.100$ & $<.100$ & $<.100$ & $<.800$ & $<.100$ & $<1.00$ & $<10.0$ & 5.00 & $<1.00$ \\
\hline \multicolumn{11}{|c|}{014721854 (site 3) Pickering Creek at Merlin, Pa. (Lat 4006 25n Long 075 35 34w) } \\
\hline $10 / 16 / 97$ & $<.200$ & $<.200$ & $<.200$ & $<.200$ & $<2.50$ & $<.200$ & -- & $<50.0$ & 9.10 & -- \\
\hline \multicolumn{11}{|c|}{014721884 (site 4) Pickering Creek at Charlestown Rd at Charlestown, Pa. (Lat 4005 57n Long 07533 20w) } \\
\hline $10 / 02 / 96$ & $<.100$ & $<.100$ & $<.200$ & $<.100$ & $<3.00$ & $<.100$ & $<1.00$ & $<10.0$ & 4.00 & $<1.00$ \\
\hline \multicolumn{11}{|c|}{01475300 (site 17) Darby Creek at Waterloo Mills near Devon, Pa. (Lat 4001 21n Long 07525 20w) } \\
\hline $10 / 16 / 96$ & $<.100$ & $<.100$ & .300 & $<.100$ & $<22.0$ & $<.100$ & $<1.00$ & $<10.0$ & 22.0 & $<1.00$ \\
\hline \multicolumn{11}{|c|}{01475840 (site 19) Crum Creek at Whitehorse, Pa. (Lat 3959 54n Long 07527 38w) } \\
\hline $10 / 16 / 96$ & $<.100$ & $<.100$ & $<.200$ & $<.200$ & $<4.00$ & $<.200$ & $<1.00$ & $<10.0$ & 13.0 & $<1.00$ \\
\hline \multicolumn{11}{|c|}{01476790 (site 22) East Branch Chester Creek at Green Hill, Pa. (Lat 3959 49n Long 075 35 40w) } \\
\hline $10 / 09 / 97$ & $<.400$ & $<.200$ & .850 & $<.200$ & $<2.50$ & $<.200$ & -- & $<50.0$ & 9.40 & -- \\
\hline \multicolumn{11}{|c|}{01476830 (site 23) East Branch Chester Creek at Milltown, Pa. (Lat 3958 21n Long 075 32 57w) } \\
\hline $10 / 07 / 96$ & $<.100$ & $<.300$ & .400 & $<.200$ & $<8.00$ & $<.100$ & $<1.00$ & $<10.0$ & 7.00 & $<1.00$ \\
\hline \multicolumn{11}{|c|}{01480629 (site 46) Buck Run at Doe Run, Pa. (Lat 3955 46n Long 07549 24w) } \\
\hline $11 / 28 / 95$ & $<.100$ & $<.100$ & $<.100$ & $<.100$ & $<.800$ & $<.100$ & $<1.00$ & $<10.0$ & 3.00 & $<1.00$ \\
\hline \multicolumn{11}{|c|}{01480632 (site 45) Doe Run at Springdell, Pa. (Lat 39 54 25n Long 07549 42w) } \\
\hline $11 / 28 / 95$ & $<.100$ & $<.100$ & $<.100$ & $<.100$ & $<.800$ & $<.100$ & $<1.00$ & $<10.0$ & 5.00 & $<1.00$ \\
\hline \multicolumn{11}{|c|}{01480648 (site 48) East Branch Brandywine Creek near Cupola, Pa. (lat 4005 41n long 07551 14w) } \\
\hline $11 / 27 / 95$ & $<.100$ & $<.100$ & $<.100$ & $<.100$ & $<1.90$ & $<.100$ & $<1.00$ & $<10.0$ & 4.00 & $<1.00$ \\
\hline \multicolumn{11}{|c|}{01480653 (site 42) East Branch Brandywine Creek at Glenmoore, Pa. (Lat 4005 48n Long 07546 44w) } \\
\hline $12 / 01 / 95$ & $<.100$ & $<.100$ & $<.100$ & $<.100$ & $<.800$ & $<.100$ & $<1.00$ & $<10.0$ & $<1.00$ & $<1.00$ \\
\hline \multicolumn{11}{|c|}{01480656 (site 47) Indian Run near Springton, Pa. (Lat 4004 33n Long 07546 52w) } \\
\hline $11 / 27 / 95$ & $<.100$ & $<.100$ & $<.100$ & $<.100$ & $<4.70$ & $<.100$ & $<1.00$ & $<10.0$ & 10.0 & $<1.00$ \\
\hline \multicolumn{11}{|c|}{01480903 (site 44) Valley Creek at Mullsteins Meadows near Downingtown, Pa. (Lat 3958 31n Long 07539 48w) } \\
\hline $11 / 21 / 95$ & $<.100$ & $<.100$ & $<.200$ & $<.100$ & $<13.0$ & $<.100$ & $<1.00$ & $<10.0$ & 30.0 & $<1.00$ \\
\hline \multicolumn{11}{|c|}{01481030 (site 40) Brandywine Creek near Chadds Ford, Pa. (Lat 3951 15n Long 07535 58w) } \\
\hline $11 / 13 / 97$ & $<.200$ & $<.200$ & .210 & $<.200$ & $<2.50$ & $<.200$ & -- & $<50.0$ & 13.0 & -- \\
\hline
\end{tabular}



Table 5. Benthic-macroinvertebrate data

[-, not found]

01472080 (Site 10) - Pigeon Creek near Parker Ford, Pa.

\begin{tabular}{|c|c|c|c|c|c|c|}
\hline Date & \multicolumn{2}{|c|}{$11 / 22 / 95$} & \multicolumn{2}{|c|}{$11 / 06 / 96$} & \multicolumn{2}{|c|}{$10 / 30 / 97$} \\
\hline Total count & \multicolumn{2}{|c|}{930} & \multicolumn{2}{|c|}{1,685} & \multicolumn{2}{|c|}{1,918} \\
\hline Total number of taxa & \multicolumn{2}{|c|}{39} & \multicolumn{2}{|c|}{40} & \multicolumn{2}{|c|}{36} \\
\hline Organism & Count & Percent & Count & Percent & Count & Percent \\
\hline \multicolumn{7}{|l|}{ Platyhelminthes } \\
\hline \multicolumn{7}{|l|}{ Turbellaria (FLATWORMS) } \\
\hline \multicolumn{7}{|l|}{ Tricladida } \\
\hline Planariidae & 7 & $0.75 \%$ & 1 & $0.06 \%$ & 2 & $0.10 \%$ \\
\hline \multicolumn{7}{|l|}{ Nemertea (PROBOSCIS WORMS) } \\
\hline \multicolumn{7}{|l|}{ Enopla } \\
\hline \multicolumn{7}{|l|}{ Hoplonemertea } \\
\hline \multicolumn{7}{|l|}{ Tetrastemmatidae } \\
\hline Prostoma & 1 & $.11 \%$ & 1 & $.06 \%$ & - & \\
\hline \multicolumn{7}{|l|}{ Annelida (SEGMENTED WORMS) } \\
\hline Oligochaeta & - & & 3 & $.18 \%$ & - & \\
\hline \multicolumn{7}{|l|}{ Tubificida } \\
\hline Naididae & 20 & $2.15 \%$ & 12 & $.71 \%$ & - & \\
\hline Arthropoda & & & & & & \\
\hline Acariformes & & & & & & \\
\hline Hydrachnidia (WATER MITES) & 24 & $2.58 \%$ & 24 & $1.42 \%$ & 116 & $6.04 \%$ \\
\hline Crustacea & & & & & & \\
\hline Amphipoda (SCUDS) & & & & & & \\
\hline Gammaridae & & & & & & \\
\hline Gammarus & - & & - & & 3 & $.16 \%$ \\
\hline Decapoda (CRAYFISH) & & & & & & \\
\hline Cambaridae & - & & 1 & $.06 \%$ & - & \\
\hline Insecta & & & & & & \\
\hline Ephemeroptera (MAYFLIES) & & & & & & \\
\hline Baetidae & & & & & & \\
\hline Baetis & 3 & $.32 \%$ & 4 & $.24 \%$ & 6 & $.31 \%$ \\
\hline Ephemerellidae & & & & & & \\
\hline Ephemerella & 270 & $29.03 \%$ & 100 & $5.93 \%$ & 482 & $25.12 \%$ \\
\hline Heptageniidae & & & & & & \\
\hline Epeorus & 1 & $.11 \%$ & 5 & $.30 \%$ & - & \\
\hline Stenacron & - & & - & & 5 & $.26 \%$ \\
\hline Stenonema & 14 & $1.51 \%$ & 38 & $2.26 \%$ & 42 & $2.19 \%$ \\
\hline Isonychiidae & & & & & & \\
\hline Isonychia & 1 & $.11 \%$ & 2 & $.12 \%$ & 5 & $.26 \%$ \\
\hline Leptophlebiidae & & & & & & \\
\hline Paraleptophlebia & 2 & $.22 \%$ & 2 & $.12 \%$ & - & \\
\hline Leptohyphidae & & & & & & \\
\hline Tricorythodes & - & & - & & 2 & $.10 \%$ \\
\hline Plecoptera (STONEFLIES) & & & & & & \\
\hline Capniidae & & & & & & \\
\hline Allocapnia & 4 & $.43 \%$ & 79 & $4.69 \%$ & 5 & $.26 \%$ \\
\hline Chloroperlidae & - & & 2 & $.12 \%$ & - & \\
\hline Nemouridae & - & & 1 & $.06 \%$ & - & \\
\hline Paranemoura & - & & - & & 1 & $.05 \%$ \\
\hline Perlidae & & & & & & \\
\hline Acroneuria & 6 & $.65 \%$ & 2 & $.12 \%$ & 3 & $.16 \%$ \\
\hline Agnetina & 2 & $.22 \%$ & - & & - & \\
\hline Paragnetina & 1 & $.11 \%$ & - & & 2 & $.10 \%$ \\
\hline Taeniopterygidae & & & & & & \\
\hline Strophopteryx & 6 & $.65 \%$ & 15 & $.89 \%$ & - & \\
\hline Taeniopteryx & - & & - & & 25 & $1.30 \%$ \\
\hline
\end{tabular}


Table 5. Benthic-macroinvertebrate data-Continued

01472080 (Site 10) - Pigeon Creek near Parker Ford, Pa._Continued

\begin{tabular}{|c|c|c|c|c|c|c|}
\hline Date & \multicolumn{2}{|c|}{$11 / 22 / 95$} & \multicolumn{2}{|c|}{$11 / 06 / 96$} & \multicolumn{2}{|c|}{$10 / 30 / 97$} \\
\hline Total count & \multicolumn{2}{|c|}{930} & \multicolumn{2}{|c|}{1,685} & \multicolumn{2}{|c|}{1,918} \\
\hline Total number of taxa & \multicolumn{2}{|c|}{39} & \multicolumn{2}{|c|}{40} & \multicolumn{2}{|c|}{36} \\
\hline Organism & Count & Percent & Count & Percent & Count & Percent \\
\hline \multicolumn{7}{|l|}{ Megaloptera } \\
\hline \multicolumn{7}{|c|}{ Corydalidae (FISHFLIES AND DOBSONFLIES) } \\
\hline Corydalus & - & & 1 & $0.06 \%$ & - & \\
\hline \multicolumn{7}{|l|}{ Trichoptera (CADDISFLIES) } \\
\hline \multicolumn{7}{|l|}{ Apataniidae } \\
\hline Apatania & 5 & $0.54 \%$ & - & & 8 & $0.42 \%$ \\
\hline \multicolumn{7}{|l|}{ Brachycentridae } \\
\hline Micrasema & 19 & $2.04 \%$ & 10 & $.59 \%$ & 55 & $2.87 \%$ \\
\hline \multicolumn{7}{|l|}{ Glossosomatidae } \\
\hline Glossosoma & 2 & $.22 \%$ & 8 & $.47 \%$ & 14 & $.73 \%$ \\
\hline \multicolumn{7}{|l|}{ Goeridae } \\
\hline Goera & 5 & $.54 \%$ & - & & 4 & $.21 \%$ \\
\hline \multicolumn{7}{|l|}{ Hydropsychidae } \\
\hline Ceratopsyche & 110 & $11.83 \%$ & 41 & $2.43 \%$ & 151 & $7.87 \%$ \\
\hline Cheumatopsyche & 32 & $3.44 \%$ & 27 & $1.60 \%$ & 80 & $4.17 \%$ \\
\hline Hydropsyche & 12 & $1.29 \%$ & 5 & $.30 \%$ & 74 & $3.86 \%$ \\
\hline \multicolumn{7}{|l|}{ Hydroptilidae } \\
\hline Hydroptila & 5 & $.54 \%$ & 2 & $.12 \%$ & 91 & $4.74 \%$ \\
\hline Leucotrichia & 2 & $.22 \%$ & 2 & $.12 \%$ & 3 & $.16 \%$ \\
\hline \multicolumn{7}{|l|}{ Philopotamidae } \\
\hline Chimarra & 32 & $3.44 \%$ & 10 & $.59 \%$ & 14 & $.73 \%$ \\
\hline \multicolumn{7}{|l|}{ Polycentropodidae } \\
\hline Nyctiophylax & - & & - & & 1 & $.05 \%$ \\
\hline Polycentropus & - & & 1 & $.06 \%$ & 1 & $.05 \%$ \\
\hline Psychomyiidae & & & & & & \\
\hline Psychomyia & 2 & $.22 \%$ & 12 & $.71 \%$ & 18 & $.94 \%$ \\
\hline Rhyacophilidae & & & & & & \\
\hline Rhyacophila & 1 & $.11 \%$ & 1 & $.06 \%$ & 18 & $.94 \%$ \\
\hline Coleoptera (BEETLES) & & & & & & \\
\hline Elmidae (RIFFLE BEETLES) & & & & & & \\
\hline Ancyronyx & 1 & $.11 \%$ & - & & - & \\
\hline Dubiraphia & 1 & $.11 \%$ & 1 & $.06 \%$ & - & \\
\hline Macronychus & - & & 4 & $.24 \%$ & - & \\
\hline Optioservus & 34 & $3.66 \%$ & 11 & $.65 \%$ & 45 & $2.34 \%$ \\
\hline Oulimnius & 2 & $.22 \%$ & 10 & $.59 \%$ & - & \\
\hline Promoresia & 1 & $.11 \%$ & - & & 1 & $.05 \%$ \\
\hline Stenelmis & 2 & $.22 \%$ & 1 & $.06 \%$ & 3 & $.16 \%$ \\
\hline Psephenidae (WATER PENN & & & & & & \\
\hline Ectopria & 2 & $.22 \%$ & 1 & $.06 \%$ & - & \\
\hline Psephenus & 2 & $.22 \%$ & 1 & $.06 \%$ & 5 & $.26 \%$ \\
\hline Diptera (TRUE FLIES) & & & & & & \\
\hline Chironomidae (MIDGES) & 223 & $23.98 \%$ & 1200 & $71.22 \%$ & 380 & $19.80 \%$ \\
\hline Empididae (DANCE FLIES) & & & & & & \\
\hline Hemerodromia & 18 & $1.94 \%$ & 6 & $.36 \%$ & 7 & $.36 \%$ \\
\hline Simuliidae (BLACK FLIES) & & & & & & \\
\hline Simulium & 4 & $.43 \%$ & 6 & $.36 \%$ & 3 & $.16 \%$ \\
\hline Tipulidae (CRANE FLIES) & & & & & & \\
\hline Antocha & 51 & $5.48 \%$ & 32 & $1.90 \%$ & 243 & $12.66 \%$ \\
\hline
\end{tabular}


Table 5. Benthic-macroinvertebrate data-Continued

01472109 (Site 6) - Stony Run near Spring City, Pa.

\begin{tabular}{|c|c|c|c|c|}
\hline Date & \multicolumn{2}{|c|}{$11 / 13 / 95$} & \multicolumn{2}{|c|}{$11 / 06 / 96$} \\
\hline Total count & \multicolumn{2}{|c|}{744} & \multicolumn{2}{|c|}{1,860} \\
\hline Total number of taxa & \multicolumn{2}{|c|}{39} & \multicolumn{2}{|c|}{34} \\
\hline Organism & Count & Percent & Count & Percent \\
\hline \multicolumn{5}{|l|}{ Platyhelminthes } \\
\hline \multicolumn{5}{|l|}{ Turbellaria (FLATWORMS) } \\
\hline \multicolumn{5}{|l|}{ Tricladida } \\
\hline Planariidae & 5 & $0.67 \%$ & 4 & $0.22 \%$ \\
\hline Nematoda (NEMATODES) & - & & 1 & $.05 \%$ \\
\hline \multicolumn{5}{|l|}{ Nemertea (PROBOSCIS WORMS) } \\
\hline \multicolumn{5}{|l|}{ Enopla } \\
\hline \multicolumn{5}{|l|}{ Hoplonemertea } \\
\hline \multicolumn{5}{|l|}{ Tetrastemmatidae } \\
\hline Prostoma & - & & 3 & $.16 \%$ \\
\hline \multicolumn{5}{|l|}{ Mollusca } \\
\hline \multicolumn{5}{|l|}{ Bivalvia (CLAMS) } \\
\hline \multicolumn{5}{|l|}{ Veneroida } \\
\hline Sphaeriidae & 2 & $.27 \%$ & 2 & $.11 \%$ \\
\hline \multicolumn{5}{|l|}{ Annelida (SEGMENTED WORMS) } \\
\hline \multicolumn{5}{|l|}{ Oligochaeta } \\
\hline \multicolumn{5}{|l|}{ Tubificida } \\
\hline Naididae & 19 & $2.55 \%$ & - & \\
\hline \multicolumn{5}{|l|}{ Arthropoda } \\
\hline Acariformes & & & & \\
\hline Hydrachnidia (WATER MITES) & 23 & $3.09 \%$ & 207 & $11.13 \%$ \\
\hline Crustacea & & & & \\
\hline Cyclopoida & 4 & $.54 \%$ & - & \\
\hline Cyclopidae & 1 & $.13 \%$ & - & \\
\hline Podocopa (SEED SHRIMP) & - & & 1 & $.05 \%$ \\
\hline Insecta & & & & \\
\hline Ephemeroptera (MAYFLIES) & & & & \\
\hline Baetidae & & & & \\
\hline Baetis & 51 & $6.85 \%$ & 143 & $7.69 \%$ \\
\hline Caenidae & & & & \\
\hline Caenis & 31 & $4.17 \%$ & 4 & $.22 \%$ \\
\hline Ephemerellidae & & & & \\
\hline Ephemerella & 32 & $4.30 \%$ & 36 & $1.94 \%$ \\
\hline Ephemeridae & & & & \\
\hline Ephemera & 1 & $.13 \%$ & - & \\
\hline Heptageniidae & & & & \\
\hline Stenacron & 3 & $.40 \%$ & - & \\
\hline Stenonema & 69 & $9.27 \%$ & 46 & $2.47 \%$ \\
\hline Isonychiidae & & & & \\
\hline Isonychia & 1 & $.13 \%$ & - & \\
\hline Leptophlebiidae & & & & \\
\hline Paraleptophlebia & 103 & $13.84 \%$ & 44 & $2.37 \%$ \\
\hline Plecoptera (STONEFLIES) & & & & \\
\hline Capniidae & & & & \\
\hline Allocapnia & 24 & $3.23 \%$ & 81 & $4.35 \%$ \\
\hline Chloroperlidae & 13 & $1.75 \%$ & 4 & $.22 \%$ \\
\hline Nemouridae & - & & 12 & $.65 \%$ \\
\hline Taeniopterygidae & & & & \\
\hline Taeniopteryx & 1 & $.13 \%$ & 9 & $.48 \%$ \\
\hline
\end{tabular}


Table 5. Benthic-macroinvertebrate data-Continued

01472109 (Site 6) - Stony Run near Spring City, Pa. —Continued

\begin{tabular}{|c|c|c|c|c|}
\hline Date & \multicolumn{2}{|c|}{$11 / 13 / 95$} & \multicolumn{2}{|c|}{$11 / 06 / 96$} \\
\hline Total count & \multicolumn{2}{|c|}{744} & \multicolumn{2}{|c|}{1,860} \\
\hline Total number of taxa & \multicolumn{2}{|c|}{39} & \multicolumn{2}{|c|}{34} \\
\hline Organism & Count & Percent & Count & Percent \\
\hline \multicolumn{5}{|l|}{ Megaloptera } \\
\hline \multicolumn{5}{|l|}{ Sialidae (ALDERFLIES) } \\
\hline Sialis & 1 & $0.13 \%$ & - & \\
\hline \multicolumn{5}{|l|}{ Trichoptera (CADDISFLIES) } \\
\hline \multicolumn{5}{|l|}{ Glossosomatidae } \\
\hline Glossosoma & - & & 3 & $0.16 \%$ \\
\hline \multicolumn{5}{|l|}{ Hydropsychidae } \\
\hline Ceratopsyche & 10 & $1.34 \%$ & 43 & $2.31 \%$ \\
\hline Cheumatopsyche & 62 & $8.33 \%$ & 71 & $3.82 \%$ \\
\hline Hydropsyche & 23 & $3.09 \%$ & 111 & $5.97 \%$ \\
\hline \multicolumn{5}{|l|}{ Hydroptilidae } \\
\hline Hydroptila & 5 & $.67 \%$ & 15 & $.81 \%$ \\
\hline \multicolumn{5}{|l|}{ Philopotamidae } \\
\hline Chimarra & 61 & $8.20 \%$ & 56 & $3.01 \%$ \\
\hline \multicolumn{5}{|l|}{ Polycentropodidae } \\
\hline Nyctiophylax & 5 & $.67 \%$ & 28 & $1.51 \%$ \\
\hline Polycentropus & 8 & $1.08 \%$ & - & \\
\hline \multicolumn{5}{|l|}{ Psychomyiidae } \\
\hline Psychomyia & - & & 8 & $.43 \%$ \\
\hline \multicolumn{5}{|l|}{ Uenoidae } \\
\hline Neophylax & 5 & $.67 \%$ & 11 & $.59 \%$ \\
\hline \multicolumn{5}{|l|}{ Coleoptera (BEETLES) } \\
\hline \multicolumn{5}{|l|}{ Elmidae (RIFFLE BEETLES) } \\
\hline Ancyronyx & 1 & $.13 \%$ & - & \\
\hline Dubiraphia & 7 & $.94 \%$ & 2 & $.11 \%$ \\
\hline Macronychus & 1 & $.13 \%$ & - & \\
\hline Optioservus & 20 & $2.69 \%$ & 71 & $3.82 \%$ \\
\hline Stenelmis & 9 & $1.21 \%$ & 5 & $.27 \%$ \\
\hline \multicolumn{5}{|c|}{ Psephenidae (WATER PENNIES) } \\
\hline Ectopria & 2 & $.27 \%$ & - & \\
\hline Psephenus & 3 & $.40 \%$ & 2 & $.11 \%$ \\
\hline \multicolumn{5}{|l|}{ Diptera (TRUE FLIES) } \\
\hline \multicolumn{5}{|l|}{ Athericidae } \\
\hline Atherix & 1 & $.13 \%$ & 1 & $.05 \%$ \\
\hline Chironomidae (MIDGES) & 125 & $16.80 \%$ & 610 & $32.80 \%$ \\
\hline \multicolumn{5}{|l|}{ Empididae (DANCE FLIES) } \\
\hline Hemerodromia & 1 & $.13 \%$ & 11 & $.59 \%$ \\
\hline \multicolumn{5}{|l|}{ Simuliidae (BLACK FLIES) } \\
\hline Simulium & 1 & $.13 \%$ & 109 & $5.86 \%$ \\
\hline \multicolumn{5}{|l|}{ Tipulidae (CRANE FLIES) } \\
\hline Antocha & 6 & $.81 \%$ & 99 & $5.32 \%$ \\
\hline Dicranota & 4 & $.54 \%$ & 7 & $.38 \%$ \\
\hline
\end{tabular}


Table 5. Benthic-macroinvertebrate data-Continued

01472138 (Site 13) - French Creek near Coventryville, Pa.

\begin{tabular}{|c|c|c|c|c|}
\hline Date & \multicolumn{2}{|c|}{$11 / 06 / 95$} & \multicolumn{2}{|c|}{ 10/01/97 } \\
\hline Total count & \multicolumn{2}{|c|}{414} & \multicolumn{2}{|c|}{1,080} \\
\hline Total number of taxa & \multicolumn{2}{|c|}{34} & \multicolumn{2}{|c|}{32} \\
\hline Organism & Count & t Percent & Count & Percent \\
\hline \multicolumn{5}{|l|}{ Platyhelminthes } \\
\hline \multicolumn{5}{|l|}{ Turbellaria (FLATWORMS) } \\
\hline \multicolumn{5}{|l|}{ Tricladida } \\
\hline Planariidae & 1 & $0.24 \%$ & 1 & $0.09 \%$ \\
\hline \multicolumn{5}{|l|}{ Nemertea (PROBOSCIS WORMS) } \\
\hline \multicolumn{5}{|l|}{ Enopla } \\
\hline \multicolumn{5}{|l|}{ Hoplonemertea } \\
\hline \multicolumn{5}{|l|}{ Tetrastemmatidae } \\
\hline Prostoma & 1 & $.24 \%$ & - & \\
\hline \multicolumn{5}{|l|}{ Mollusca } \\
\hline \multicolumn{5}{|l|}{ Gastropoda (SNAILS) } \\
\hline \multicolumn{5}{|l|}{ Basommatophora } \\
\hline \multicolumn{5}{|l|}{ Ancylidae } \\
\hline Ferrissia & 7 & $1.69 \%$ & 22 & $2.04 \%$ \\
\hline \multicolumn{5}{|l|}{ Annelida (SEGMENTED WORMS) } \\
\hline \multicolumn{5}{|l|}{ Oligochaeta } \\
\hline Tubificida & & & & \\
\hline Naididae & 7 & $1.69 \%$ & - & \\
\hline Tubificidae & 1 & $.24 \%$ & - & \\
\hline Arthropoda & & & & \\
\hline Acariformes & & & & \\
\hline Hydrachnidia (WATER MITES) & 80 & $19.32 \%$ & 83 & $7.69 \%$ \\
\hline Crustacea & & & & \\
\hline Podocopa (SEED SHRIMP) & 1 & $.24 \%$ & - & \\
\hline Insecta & & & & \\
\hline Ephemeroptera (MAYFLIES) & & & & \\
\hline Baetidae & & & & \\
\hline Baetis & - & & 6 & $.56 \%$ \\
\hline Pseudocloeon & - & & 8 & $.74 \%$ \\
\hline Caenidae & & & & \\
\hline Caenis & 1 & $.24 \%$ & 1 & $.09 \%$ \\
\hline Ephemerellidae & & & & \\
\hline Ephemerella & 6 & $1.45 \%$ & - & \\
\hline Heptageniidae & & & & \\
\hline Stenonema & 13 & $3.14 \%$ & 33 & $3.06 \%$ \\
\hline Isonychiidae & & & & \\
\hline Isonychia & 11 & $2.66 \%$ & 20 & $1.85 \%$ \\
\hline Odonata (DRAGONFLIES AND DA & & & & \\
\hline Coenagrionidae & & & & \\
\hline Argia & 1 & $.24 \%$ & 一 & \\
\hline Gomphidae & 1 & $.24 \%$ & - & \\
\hline Plecoptera (STONEFLIES) & & & & \\
\hline Capniidae & & & & \\
\hline Allocapnia & 2 & $.48 \%$ & - & \\
\hline Perlidae & & & & \\
\hline Acroneuria & - & & 7 & $.65 \%$ \\
\hline Taeniopterygidae & & & & \\
\hline Strophopteryx & 20 & $4.83 \%$ & 2 & $.19 \%$ \\
\hline Taeniopteryx & 11 & $2.66 \%$ & - & \\
\hline Hemiptera (TRUE BUGS) & & & & \\
\hline Veliidae & & & & \\
\hline Rhagovelia & - & & 2 & $.19 \%$ \\
\hline
\end{tabular}


Table 5. Benthic-macroinvertebrate data-Continued

01472138 (Site 13) - French Creek near Coventryville, Pa.-Continued

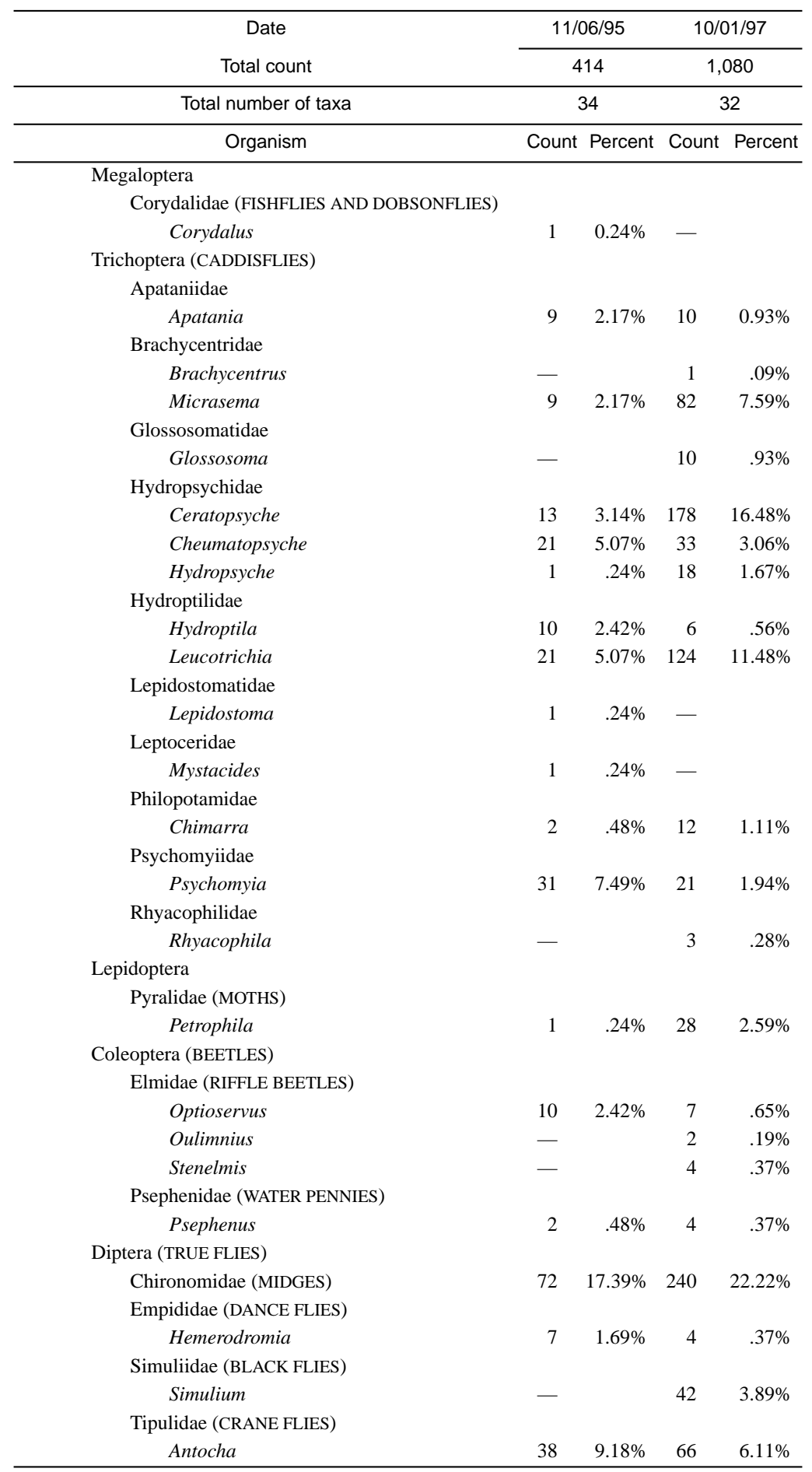


Table 5. Benthic-macroinvertebrate data-Continued

01472140 (Site 12) - South Branch French Creek at Coventryville, Pa.

\begin{tabular}{|c|c|c|c|c|}
\hline Date & \multicolumn{2}{|c|}{$11 / 06 / 95$} & \multicolumn{2}{|c|}{ 10/09/97 } \\
\hline Total count & \multicolumn{2}{|c|}{585} & \multicolumn{2}{|c|}{1,527} \\
\hline Total number of taxa & \multicolumn{2}{|c|}{32} & \multicolumn{2}{|c|}{32} \\
\hline Organism & Count & Percent & Count & Percent \\
\hline \multicolumn{5}{|l|}{ Platyhelminthes } \\
\hline \multicolumn{5}{|l|}{ Turbellaria (FLATWORMS) } \\
\hline \multicolumn{5}{|l|}{ Tricladida } \\
\hline Planariidae & 10 & $1.71 \%$ & - & \\
\hline \multicolumn{5}{|l|}{ Nemertea (PROBOSCIS WORMS) } \\
\hline \multicolumn{5}{|l|}{ Enopla } \\
\hline \multicolumn{5}{|l|}{ Hoplonemertea } \\
\hline \multicolumn{5}{|l|}{ Tetrastemmatidae } \\
\hline Prostoma & 5 & $.85 \%$ & - & \\
\hline \multicolumn{5}{|l|}{ Mollusca } \\
\hline \multicolumn{5}{|l|}{ Gastropoda (SNAILS) } \\
\hline \multicolumn{5}{|l|}{ Basommatophora } \\
\hline \multicolumn{5}{|l|}{ Ancylidae } \\
\hline Ferrissia & 3 & $.51 \%$ & 1 & $0.07 \%$ \\
\hline \multicolumn{5}{|l|}{ Planorbidae } \\
\hline Gyraulus & - & & 1 & $.07 \%$ \\
\hline \multicolumn{5}{|l|}{ Annelida (SEGMENTED WORMS) } \\
\hline \multicolumn{5}{|l|}{ Oligochaeta } \\
\hline Lumbriculida & & & & \\
\hline Lumbriculidae & 1 & $.17 \%$ & - & \\
\hline Tubificida & & & & \\
\hline Naididae & 6 & $1.03 \%$ & - & \\
\hline Arthropoda & & & & \\
\hline Acariformes & & & & \\
\hline Hydrachnidia (WATER MITES) & 32 & $5.47 \%$ & 12 & $.79 \%$ \\
\hline Crustacea & & & & \\
\hline Cyclopoida & 3 & $.51 \%$ & - & \\
\hline Isopoda (SOW BUGS) & & & & \\
\hline Asellidae & & & & \\
\hline Caecidotea & 1 & $.17 \%$ & - & \\
\hline Podocopa (SEED SHRIMP) & - & & 1 & $.07 \%$ \\
\hline Insecta & & & & \\
\hline Ephemeroptera (MAYFLIES) & & & & \\
\hline Baetidae & & & & \\
\hline Baetis & - & & 5 & $.33 \%$ \\
\hline Pseudocloeon & - & & 27 & $1.77 \%$ \\
\hline Ephemerellidae & & & & \\
\hline Ephemerella & 45 & $7.69 \%$ & 17 & $1.11 \%$ \\
\hline Heptageniidae & & & & \\
\hline Epeorus & 6 & $1.03 \%$ & 19 & $1.24 \%$ \\
\hline Stenonema & 8 & $1.37 \%$ & 45 & $2.95 \%$ \\
\hline Isonychiidae & & & & \\
\hline Isonychia & 15 & $2.56 \%$ & 16 & $1.05 \%$ \\
\hline Plecoptera (STONEFLIES) & & & & \\
\hline Capniidae & & & & \\
\hline Allocapnia & 1 & $.17 \%$ & - & \\
\hline Chloroperlidae & - & & 3 & $.20 \%$ \\
\hline Taeniopterygidae & & & & \\
\hline Strophopteryx & 70 & $11.97 \%$ & - & \\
\hline Taeniopteryx & 5 & $.85 \%$ & 20 & $1.31 \%$ \\
\hline
\end{tabular}


Table 5. Benthic-macroinvertebrate data-Continued

01472140 (Site 12) - South Branch French Creek at Coventryville, Pa.-Continued

\begin{tabular}{|c|c|c|c|c|}
\hline Date & \multicolumn{2}{|c|}{$11 / 06 / 95$} & \multicolumn{2}{|c|}{ 10/09/97 } \\
\hline Total count & \multicolumn{2}{|c|}{585} & \multicolumn{2}{|c|}{1,527} \\
\hline Total number of taxa & \multicolumn{2}{|c|}{32} & \multicolumn{2}{|c|}{32} \\
\hline Organism & Count & Percent & Count & Percent \\
\hline \multicolumn{5}{|l|}{ Megaloptera } \\
\hline \multicolumn{5}{|c|}{ Corydalidae (FISHFLIES AND DOBSONFLIES) } \\
\hline Corydalus & - & & 3 & $0.20 \%$ \\
\hline \multicolumn{5}{|l|}{ Trichoptera (CADDISFLIES) } \\
\hline \multicolumn{5}{|l|}{ Apataniidae } \\
\hline Apatania & 3 & $0.51 \%$ & 6 & $.39 \%$ \\
\hline \multicolumn{5}{|l|}{ Brachycentridae } \\
\hline Micrasema & 21 & $3.59 \%$ & 5 & $.33 \%$ \\
\hline \multicolumn{5}{|l|}{ Glossosomatidae } \\
\hline Glossosoma & - & & 17 & $1.11 \%$ \\
\hline \multicolumn{5}{|l|}{ Hydropsychidae } \\
\hline Ceratopsyche & 15 & $2.56 \%$ & 409 & $26.78 \%$ \\
\hline Cheumatopsyche & 80 & $13.68 \%$ & 44 & $2.88 \%$ \\
\hline Hydropsyche & 13 & $2.22 \%$ & 74 & $4.85 \%$ \\
\hline \multicolumn{5}{|l|}{ Hydroptilidae } \\
\hline Leucotrichia & 7 & $1.20 \%$ & 408 & $26.72 \%$ \\
\hline \multicolumn{5}{|l|}{ Philopotamidae } \\
\hline Chimarra & - & & 18 & $1.18 \%$ \\
\hline \multicolumn{5}{|l|}{ Polycentropodidae } \\
\hline Neureclipsis & - & & 1 & $.07 \%$ \\
\hline Nyctiophylax & 1 & $.17 \%$ & - & \\
\hline \multicolumn{5}{|l|}{ Psychomyiidae } \\
\hline Psychomyia & 8 & $1.37 \%$ & 14 & $.92 \%$ \\
\hline \multicolumn{5}{|l|}{ Rhyacophilidae } \\
\hline Rhyacophila & 1 & $.17 \%$ & - & \\
\hline \multicolumn{5}{|l|}{ Lepidoptera } \\
\hline \multicolumn{5}{|l|}{ Pyralidae (MOTHS) } \\
\hline Petrophila & 5 & $.85 \%$ & 12 & $.79 \%$ \\
\hline \multicolumn{5}{|l|}{ Coleoptera (BEETLES) } \\
\hline \multicolumn{5}{|l|}{ Elmidae (RIFFLE BEETLES) } \\
\hline Ancyronyx & - & & 2 & $.13 \%$ \\
\hline Optioservus & 15 & $2.56 \%$ & 17 & $1.11 \%$ \\
\hline Oulimnius & - & & 2 & $.13 \%$ \\
\hline Promoresia & 1 & $.17 \%$ & - & \\
\hline Stenelmis & 7 & $1.20 \%$ & 10 & $.65 \%$ \\
\hline \multicolumn{5}{|l|}{ Diptera (TRUE FLIES) } \\
\hline Chironomidae (MIDGES) & 110 & $18.80 \%$ & 204 & $13.36 \%$ \\
\hline Empididae (DANCE FLIES) & & & & \\
\hline Hemerodromia & 6 & $1.03 \%$ & 2 & $.13 \%$ \\
\hline Simuliidae (BLACKFLIES) & & & & \\
\hline Simulium & 36 & $6.15 \%$ & 64 & $4.19 \%$ \\
\hline Tipulidae (CRANE FLIES) & & & & \\
\hline Antocha & 45 & $7.69 \%$ & 48 & $3.14 \%$ \\
\hline
\end{tabular}


Table 5. Benthic-macroinvertebrate data-Continued

01472154 (Site 14) - French Creek near Pughtown, Pa.

\begin{tabular}{|c|c|c|c|c|}
\hline Date & \multicolumn{2}{|c|}{$11 / 06 / 95$} & \multicolumn{2}{|c|}{$10 / 08 / 97$} \\
\hline Total count & \multicolumn{2}{|c|}{388} & \multicolumn{2}{|c|}{800} \\
\hline Total number of taxa & \multicolumn{2}{|c|}{34} & \multicolumn{2}{|c|}{33} \\
\hline Organism & Count & Percent & Count & Percent \\
\hline \multicolumn{5}{|l|}{ Platyhelminthes } \\
\hline \multicolumn{5}{|l|}{ Turbellaria (FLATWORMS) } \\
\hline \multicolumn{5}{|l|}{ Tricladida } \\
\hline Planariidae & 3 & $0.77 \%$ & 2 & $0.25 \%$ \\
\hline \multicolumn{5}{|l|}{ Mollusca } \\
\hline \multicolumn{5}{|l|}{ Bivalvia (CLAMS) } \\
\hline \multicolumn{5}{|l|}{ Veneroida } \\
\hline Sphaeriidae & - & & 41 & $5.13 \%$ \\
\hline \multicolumn{5}{|l|}{ Annelida (SEGMENTED WORMS) } \\
\hline \multicolumn{5}{|l|}{ Oligochaeta } \\
\hline \multicolumn{5}{|l|}{ Lumbriculida } \\
\hline Lumbriculidae & 1 & $.26 \%$ & 1 & $.13 \%$ \\
\hline \multicolumn{5}{|l|}{ Tubificida } \\
\hline Naididae & 2 & $.52 \%$ & - & \\
\hline \multicolumn{5}{|l|}{ Arthropoda } \\
\hline \multicolumn{5}{|l|}{ Acariformes } \\
\hline Hydrachnidia (WATER MITES) & 19 & $4.90 \%$ & 7 & $.88 \%$ \\
\hline \multicolumn{5}{|l|}{ Insecta } \\
\hline Ephemeroptera (MAYFLIES) & & & & \\
\hline Baetidae & & & & \\
\hline Baetis & 2 & $.52 \%$ & 16 & $2.00 \%$ \\
\hline Pseudocloeon & 2 & $.52 \%$ & 3 & $.38 \%$ \\
\hline Caenidae & & & & \\
\hline Caenis & 4 & $1.03 \%$ & - & \\
\hline Ephemerellidae & & & & \\
\hline Ephemerella & 28 & $7.22 \%$ & 6 & $.75 \%$ \\
\hline Heptageniidae & & & & \\
\hline Epeorus & 4 & $1.03 \%$ & - & \\
\hline Stenonema & 26 & $6.70 \%$ & 24 & $3.00 \%$ \\
\hline Isonychiidae & & & & \\
\hline Isonychia & 12 & $3.09 \%$ & 6 & $.75 \%$ \\
\hline Plecoptera (STONEFLIES) & & & & \\
\hline Capniidae & & & & \\
\hline Allocapnia & 15 & $3.87 \%$ & 2 & $.25 \%$ \\
\hline Chloroperlidae & 1 & $.26 \%$ & - & \\
\hline Nemouridae & 1 & $.26 \%$ & - & \\
\hline Perlidae & & & & \\
\hline Acronuria & - & & 1 & $.13 \%$ \\
\hline Agnetina & 1 & $.26 \%$ & - & \\
\hline Paragnetina & - & & 1 & $.13 \%$ \\
\hline Taeniopterygidae & & & & \\
\hline Strophopteryx & 35 & $9.02 \%$ & - & \\
\hline Taeniopteryx & 15 & $3.87 \%$ & 2 & $.25 \%$ \\
\hline Hemiptera (TRUE BUGS) & & & & \\
\hline Veliidae & & & & \\
\hline Rhagovelia & - & & 1 & $.13 \%$ \\
\hline Megaloptera & & & & \\
\hline Corydalidae (FISHFLIES ANL & & & & \\
\hline Corydalus & 1 & $.26 \%$ & 3 & $.38 \%$ \\
\hline Nigronia & - & & 1 & $.13 \%$ \\
\hline
\end{tabular}


Table 5. Benthic-macroinvertebrate data-Continued

01472154 (Site 14) - French Creek near Pughtown, Pa.-Continued

\begin{tabular}{|c|c|c|c|c|}
\hline Date & \multicolumn{2}{|c|}{$11 / 06 / 95$} & \multicolumn{2}{|c|}{$10 / 08 / 97$} \\
\hline Total count & \multicolumn{2}{|c|}{388} & \multicolumn{2}{|c|}{800} \\
\hline Total number of taxa & \multicolumn{2}{|c|}{34} & \multicolumn{2}{|c|}{33} \\
\hline Organism & Count & Percent & Count & Percent \\
\hline \multicolumn{5}{|l|}{ Trichoptera (CADDISFLIES) } \\
\hline \multicolumn{5}{|l|}{ Apataniidae } \\
\hline Apatania & 3 & $0.77 \%$ & - & \\
\hline \multicolumn{5}{|l|}{ Brachycentridae } \\
\hline Micrasema & 6 & $1.55 \%$ & 58 & $7.25 \%$ \\
\hline \multicolumn{5}{|l|}{ Glossosomatidae } \\
\hline Glossosoma & - & & 8 & $1.00 \%$ \\
\hline \multicolumn{5}{|l|}{ Helicopsychidae } \\
\hline Helicopsyche & 3 & $.77 \%$ & - & \\
\hline \multicolumn{5}{|l|}{ Hydropsychidae } \\
\hline Ceratopsyche & 13 & $3.35 \%$ & 260 & $32.50 \%$ \\
\hline Cheumatopsyche & 22 & $5.67 \%$ & 28 & $3.50 \%$ \\
\hline Hydropsyche & 1 & $.26 \%$ & 57 & $7.13 \%$ \\
\hline Macrostemum & - & & 1 & $.13 \%$ \\
\hline \multicolumn{5}{|l|}{ Hydroptilidae } \\
\hline Hydroptila & - & & 1 & $.13 \%$ \\
\hline Leucotrichia & 55 & $14.18 \%$ & 26 & $3.25 \%$ \\
\hline \multicolumn{5}{|l|}{ Philopotamidae } \\
\hline Chimarra & 6 & $1.55 \%$ & 37 & $4.63 \%$ \\
\hline \multicolumn{5}{|l|}{ Polycentropodidae } \\
\hline Neureclipsis & 1 & $.26 \%$ & 2 & $.25 \%$ \\
\hline \multicolumn{5}{|l|}{ Psychomyiidae } \\
\hline Psychomyia & 7 & $1.80 \%$ & 90 & $11.25 \%$ \\
\hline \multicolumn{5}{|l|}{ Lepidoptera } \\
\hline \multicolumn{5}{|l|}{ Pyralidae (MOTHS) } \\
\hline Petrophila & 3 & $.77 \%$ & 5 & $.63 \%$ \\
\hline \multicolumn{5}{|l|}{ Coleoptera (BEETLES) } \\
\hline \multicolumn{5}{|l|}{ Elmidae (RIFFLE BEETLES) } \\
\hline Optioservus & 7 & $1.80 \%$ & 5 & $.63 \%$ \\
\hline \multicolumn{5}{|l|}{ Psephenidae (WATER PENNIES) } \\
\hline Psephenus & - & & 3 & $.38 \%$ \\
\hline \multicolumn{5}{|l|}{ Diptera (TRUE FLIES) } \\
\hline Chironomidae (MIDGES) & 82 & $21.13 \%$ & 82 & $1.25 \%$ \\
\hline \multicolumn{5}{|l|}{ Empididae (DANCE FLIES) } \\
\hline Hemerodromia & 1 & $.26 \%$ & - & \\
\hline \multicolumn{5}{|l|}{ Simuliidae (BLACK FLIES) } \\
\hline Simulium & 2 & $.52 \%$ & 2 & $.25 \%$ \\
\hline \multicolumn{5}{|l|}{ Tipulidae (CRANE FLIES) } \\
\hline Antocha & 4 & $1.03 \%$ & 18 & $2.25 \%$ \\
\hline
\end{tabular}


Table 5. Benthic-macroinvertebrate data-Continued

01472157 (Site 15) - French Creek near Phoenixville, Pa.

\begin{tabular}{|c|c|c|c|c|c|c|}
\hline Date & \multicolumn{2}{|c|}{$10 / 27 / 95$} & \multicolumn{2}{|c|}{$10 / 28 / 96$} & \multicolumn{2}{|c|}{ 10/02/97 } \\
\hline Total count & \multicolumn{2}{|c|}{281} & \multicolumn{2}{|c|}{198} & \multicolumn{2}{|c|}{1,677} \\
\hline Total number of taxa & \multicolumn{2}{|c|}{35} & \multicolumn{2}{|c|}{21} & \multicolumn{2}{|c|}{37} \\
\hline Organism & Count & Percent & Count & Percent & Count & Percent \\
\hline \multicolumn{7}{|l|}{ Platyhelminthes } \\
\hline \multicolumn{7}{|l|}{ Turbellaria (FLATWORMS) } \\
\hline \multicolumn{7}{|l|}{ Tricladida } \\
\hline Planariidae & 2 & $0.71 \%$ & - & & - & \\
\hline Nematoda (NEMATODES) & - & & - & & 2 & $0.12 \%$ \\
\hline \multicolumn{7}{|l|}{ Mollusca } \\
\hline \multicolumn{7}{|l|}{ Gastropoda (SNAILS) } \\
\hline \multicolumn{7}{|l|}{ Mesogastropoda } \\
\hline \multicolumn{7}{|l|}{ Hydrobiidae } \\
\hline Amnicola & 1 & $.36 \%$ & - & & 1 & $.06 \%$ \\
\hline Basommatophora & & & & & & \\
\hline Ancylidae & & & & & & \\
\hline Ferrissia & 2 & $.71 \%$ & 2 & $1.01 \%$ & 16 & $.95 \%$ \\
\hline Gyraulus & 3 & $1.07 \%$ & - & & - & \\
\hline Bivalvia (CLAMS) & & & & & & \\
\hline Veneroida & & & & & & \\
\hline Sphaeriidae & - & & - & & 1 & $.06 \%$ \\
\hline Annelida (SEGMENTED WORMS) & & & & & & \\
\hline Oligochaeta & & & & & & \\
\hline Tubificida & & & & & & \\
\hline Naididae & - & & 3 & $1.52 \%$ & - & \\
\hline Arthropoda & & & & & & \\
\hline Acariformes & & & & & & \\
\hline Hydrachnidia (WATER MITES) & 15 & $5.34 \%$ & 7 & $3.54 \%$ & 11 & $.66 \%$ \\
\hline Crustacea & & & & & & \\
\hline Cyclopoida & 1 & $.36 \%$ & - & & - & \\
\hline Insecta & & & & & & \\
\hline Ephemeroptera (MAYFLIES) & & & & & & \\
\hline Baetidae & & & & & & \\
\hline Baetis & 1 & $.36 \%$ & 1 & $.51 \%$ & 47 & $2.80 \%$ \\
\hline Pseudocloeon & 1 & $.36 \%$ & - & & 13 & $.78 \%$ \\
\hline Caenidae & & & & & & \\
\hline Caenis & 2 & $.71 \%$ & - & & - & \\
\hline Ephemerellidae & & & & & & \\
\hline Ephemerella & 60 & $21.35 \%$ & 9 & $4.55 \%$ & 12 & $.72 \%$ \\
\hline Heptageniidae & & & & & & \\
\hline Epeorus & 30 & $10.68 \%$ & 1 & $.51 \%$ & 20 & $1.19 \%$ \\
\hline Stenonema & 40 & $14.23 \%$ & 7 & $3.54 \%$ & 101 & $6.02 \%$ \\
\hline Isonychiidae & & & & & & \\
\hline Isonychia & 5 & $1.78 \%$ & - & & 409 & $24.39 \%$ \\
\hline Odonata (DRAGONFLIES AND DA & & & & & & \\
\hline Coenagrionidae & & & & & & \\
\hline Argia & - & & - & & 1 & $.06 \%$ \\
\hline Plecoptera (STONEFLIES) & & & & & & \\
\hline Capniidae & & & & & & \\
\hline Allocapnia & 2 & $.71 \%$ & 8 & $4.04 \%$ & - & \\
\hline Chloroperlidae & 5 & $1.78 \%$ & 1 & $.51 \%$ & - & \\
\hline Perlidae & & & & & & \\
\hline Acroneuria & 2 & $.71 \%$ & - & & 5 & $.30 \%$ \\
\hline Agnetina & 2 & $.71 \%$ & - & & 2 & $.12 \%$ \\
\hline Paragnetina & - & & - & & 45 & $2.68 \%$ \\
\hline Taeniopterygidae & & & & & & \\
\hline Strophopteryx & - & & 10 & $5.05 \%$ & - & \\
\hline Taeniopteryx & 5 & $1.78 \%$ & 2 & $1.01 \%$ & - & \\
\hline
\end{tabular}


Table 5. Benthic-macroinvertebrate data-Continued

01472157 (Site 15) - French Creek near Phoenixville, Pa.-Continued

\begin{tabular}{|c|c|c|c|c|c|c|}
\hline Date & \multicolumn{2}{|c|}{$10 / 27 / 95$} & \multicolumn{2}{|c|}{ 10/28/96 } & \multicolumn{2}{|c|}{ 10/02/97 } \\
\hline Total count & \multicolumn{2}{|c|}{281} & \multicolumn{2}{|c|}{198} & \multicolumn{2}{|c|}{1,677} \\
\hline Total number of taxa & \multicolumn{2}{|c|}{35} & \multicolumn{2}{|c|}{21} & \multicolumn{2}{|c|}{37} \\
\hline Organism & Count & Percent & Count & Percent & Count & Percent \\
\hline \multicolumn{7}{|l|}{ Megaloptera } \\
\hline \multicolumn{7}{|c|}{ Corydalidae (FISHFLIES AND DOBSONFLIES) } \\
\hline Corydalus & - & & - & & 1 & $0.06 \%$ \\
\hline \multicolumn{7}{|l|}{ Trichoptera (CADDISFLIES) } \\
\hline \multicolumn{7}{|l|}{ Apataniidae } \\
\hline Apatania & 4 & $1.42 \%$ & - & & 16 & $.95 \%$ \\
\hline \multicolumn{7}{|l|}{ Brachycentridae } \\
\hline Micrasema & 2 & $.71 \%$ & 4 & $2.02 \%$ & 87 & $5.19 \%$ \\
\hline \multicolumn{7}{|l|}{ Glossosomatidae } \\
\hline Glossosoma & 1 & $.36 \%$ & 6 & $3.03 \%$ & 47 & $2.80 \%$ \\
\hline Protoptila & 4 & $1.42 \%$ & - & & 26 & $1.55 \%$ \\
\hline \multicolumn{7}{|l|}{ Helicopsychidae } \\
\hline Helicopsyche & 2 & $.71 \%$ & - & & 6 & $.36 \%$ \\
\hline \multicolumn{7}{|l|}{ Hydropsychidae } \\
\hline Ceratopsyche & 14 & $4.98 \%$ & 15 & $7.58 \%$ & 155 & $9.24 \%$ \\
\hline Cheumatopsyche & 5 & $1.78 \%$ & 6 & $3.03 \%$ & 59 & $3.52 \%$ \\
\hline Hydropsyche & - & & 5 & $2.53 \%$ & 131 & $7.81 \%$ \\
\hline \multicolumn{7}{|l|}{ Hydroptilidae } \\
\hline Leucotrichia & 1 & $.36 \%$ & - & & 166 & $9.90 \%$ \\
\hline \multicolumn{7}{|l|}{ Lepidostomatidae } \\
\hline Lepidostoma & 4 & $1.42 \%$ & - & & 5 & $.30 \%$ \\
\hline \multicolumn{7}{|l|}{ Leptoceridae } \\
\hline Oecetis & - & & - & & 1 & $.06 \%$ \\
\hline Philopotamidae & & & & & & \\
\hline Chimarra & 16 & $5.69 \%$ & - & & 85 & $5.07 \%$ \\
\hline Wormaldia & 1 & $.36 \%$ & - & & - & \\
\hline Polycentropodidae & & & & & & \\
\hline Neureclipsis & - & & - & & 1 & $.06 \%$ \\
\hline Psychomyiidae & & & & & & \\
\hline Psychomyia & - & & 5 & $2.53 \%$ & 14 & $.83 \%$ \\
\hline Lepidoptera & & & & & & \\
\hline Pyralidae (MOTHS) & & & & & & \\
\hline Petrophila & - & & 1 & $.51 \%$ & 1 & $.06 \%$ \\
\hline Coleoptera (BEETLES) & & & & & & \\
\hline Elmidae (RIFFLE BEETLES) & & & & & & \\
\hline Optioservus & - & & - & & 6 & $.36 \%$ \\
\hline Stenelmis & - & & - & & 1 & $.06 \%$ \\
\hline Psephenidae (WATER PENN & & & & & & \\
\hline Ectopria & 1 & $.36 \%$ & - & & - & \\
\hline Psephenus & 1 & $.36 \%$ & - & & 1 & $.06 \%$ \\
\hline Diptera (TRUE FLIES) & & & & & & \\
\hline Chironomidae (MIDGES) & 40 & $14.23 \%$ & 94 & $47.47 \%$ & 140 & $8.35 \%$ \\
\hline Empididae (DANCE FLIES) & & & & & & \\
\hline Hemerodromia & 1 & $.36 \%$ & - & & - & \\
\hline Simuliidae (BLACK FLIES) & & & & & & \\
\hline Simulium & 4 & $1.42 \%$ & 7 & $3.54 \%$ & 21 & $1.25 \%$ \\
\hline Tipulidae (CRANE FLIES) & & & & & & \\
\hline Antocha & 1 & $.36 \%$ & 4 & $2.02 \%$ & 21 & $1.25 \%$ \\
\hline
\end{tabular}


Table 5. Benthic-macroinvertebrate data-Continued

014721612 (Site 16) - French Creek at Railroad Bridge at Phoenixville, Pa.

\begin{tabular}{|c|c|c|c|c|}
\hline Date & \multicolumn{2}{|c|}{$11 / 07 / 95$} & \multicolumn{2}{|c|}{ 10/02/97 } \\
\hline Total count & \multicolumn{2}{|c|}{90} & \multicolumn{2}{|c|}{1,234} \\
\hline Total number of taxa & \multicolumn{2}{|c|}{19} & \multicolumn{2}{|c|}{26} \\
\hline Organism & Count & Percent & Count & Percent \\
\hline \multicolumn{5}{|l|}{ Platyhelminthes } \\
\hline \multicolumn{5}{|l|}{ Turbellaria (FLATWORMS) } \\
\hline \multicolumn{5}{|l|}{ Tricladida } \\
\hline Planariidae & 2 & $2.22 \%$ & 10 & $0.81 \%$ \\
\hline \multicolumn{5}{|l|}{ Mollusca } \\
\hline \multicolumn{5}{|l|}{ Gastropoda (SNAILS) } \\
\hline \multicolumn{5}{|l|}{ Basommatophora } \\
\hline \multicolumn{5}{|l|}{ Ancylidae } \\
\hline Ferrissia & - & & 15 & $1.22 \%$ \\
\hline \multicolumn{5}{|l|}{ Lymnaeidae } \\
\hline Lymnaea & - & & 1 & $.08 \%$ \\
\hline \multicolumn{5}{|l|}{ Physidae } \\
\hline Physa & 1 & $1.11 \%$ & - & \\
\hline \multicolumn{5}{|l|}{ Annelida (SEGMENTED WORMS) } \\
\hline \multicolumn{5}{|l|}{ Oligochaeta } \\
\hline \multicolumn{5}{|l|}{ Tubificida } \\
\hline Naididae & 1 & $1.11 \%$ & - & \\
\hline \multicolumn{5}{|l|}{ Arthropoda } \\
\hline \multicolumn{5}{|l|}{ Acariformes } \\
\hline Hydrachnidia (WATER MITES) & 1 & $1.11 \%$ & 10 & $.81 \%$ \\
\hline \multicolumn{5}{|l|}{ Crustacea } \\
\hline \multicolumn{5}{|l|}{ Amphipoda (SCUDS) } \\
\hline Gammaridae & & & & \\
\hline Gammarus & - & & 8 & $.65 \%$ \\
\hline Insecta & & & & \\
\hline Ephemeroptera (MAYFLIES) & & & & \\
\hline Baetidae & & & & \\
\hline Baetis & - & & 27 & $2.19 \%$ \\
\hline Pseudocloeon & - & & 24 & $1.94 \%$ \\
\hline Caenidae & & & & \\
\hline Caenis & 6 & $6.67 \%$ & - & \\
\hline Ephemerellidae & & & & \\
\hline Ephemerella & 4 & $4.44 \%$ & - & \\
\hline Heptageniidae & & & & \\
\hline Stenonema & 4 & $4.44 \%$ & 2 & $.16 \%$ \\
\hline Isonychiidae & & & & \\
\hline Isonychia & - & & 5 & $.41 \%$ \\
\hline Odonata (DRAGONFLIES AND D & & & & \\
\hline Coenagrionidae & & & & \\
\hline Argia & 1 & $1.11 \%$ & - & \\
\hline Plecoptera (STONEFLIES) & & & & \\
\hline Capniidae & 1 & $1.11 \%$ & - & \\
\hline Perlidae & & & & \\
\hline Acroneuria & - & & 4 & $.32 \%$ \\
\hline Taeniopterygidae & & & & \\
\hline Taeniopteryx & 2 & $2.22 \%$ & - & \\
\hline
\end{tabular}


Table 5. Benthic-macroinvertebrate data-Continued

014721612 (Site 16) - French Creek at Railroad Bridge at Phoenixville, Pa.-Continued

\begin{tabular}{|c|c|c|c|c|}
\hline Date & \multicolumn{2}{|c|}{$11 / 07 / 95$} & \multicolumn{2}{|c|}{$10 / 02 / 97$} \\
\hline Total count & \multicolumn{2}{|c|}{90} & \multicolumn{2}{|c|}{1,234} \\
\hline Total number of taxa & \multicolumn{2}{|c|}{19} & \multicolumn{2}{|c|}{26} \\
\hline Organism & Count & Percent & Count & Percent \\
\hline \multicolumn{5}{|l|}{ Trichoptera (CADDISFLIES) } \\
\hline \multicolumn{5}{|l|}{ Apataniidae } \\
\hline Apatania & - & & 2 & $0.16 \%$ \\
\hline \multicolumn{5}{|l|}{ Brachycentridae } \\
\hline Micrasema & - & & 3 & $.24 \%$ \\
\hline \multicolumn{5}{|l|}{ Helicopsychidae } \\
\hline Helicopsyche & - & & 2 & $.16 \%$ \\
\hline \multicolumn{5}{|l|}{ Hydropsychidae } \\
\hline Ceratopsyche & 14 & $15.56 \%$ & 183 & $14.83 \%$ \\
\hline Cheumatopsyche & 6 & $6.67 \%$ & 130 & $10.53 \%$ \\
\hline Hydropsyche & 8 & $8.89 \%$ & 186 & $15.07 \%$ \\
\hline \multicolumn{5}{|l|}{ Hydroptilidae } \\
\hline Leucotrichia & - & & 12 & $.97 \%$ \\
\hline \multicolumn{5}{|l|}{ Philopotamidae } \\
\hline Chimarra & 1 & $1.11 \%$ & - & \\
\hline \multicolumn{5}{|l|}{ Psychomyiidae } \\
\hline Psychomyia & - & & 2 & $.16 \%$ \\
\hline \multicolumn{5}{|l|}{ Lepidoptera } \\
\hline \multicolumn{5}{|l|}{ Pyralidae (MOTHS) } \\
\hline Petrophila & 3 & $3.33 \%$ & 31 & $2.51 \%$ \\
\hline \multicolumn{5}{|l|}{ Coleoptera (BEETLES) } \\
\hline \multicolumn{5}{|l|}{ Elmidae (RIFFLE BEETLES) } \\
\hline Optioservus & 2 & $2.22 \%$ & 4 & $.32 \%$ \\
\hline Oulimnius & 1 & $1.11 \%$ & - & \\
\hline Promoresia & - & & 2 & $.16 \%$ \\
\hline \multicolumn{5}{|c|}{ Psephenidae (WATER PENNIES) } \\
\hline Psephenus & - & & 4 & $.32 \%$ \\
\hline \multicolumn{5}{|l|}{ Diptera (TRUE FLIES) } \\
\hline Chironomidae (MIDGES) & 31 & $34.44 \%$ & 525 & $42.54 \%$ \\
\hline \multicolumn{5}{|l|}{ Empididae (DANCE FLIES) } \\
\hline Hemerodromia & 1 & $1.11 \%$ & 1 & $.08 \%$ \\
\hline \multicolumn{5}{|l|}{ Simuliidae (BLACK FLIES) } \\
\hline Simulium & - & & 20 & $1.62 \%$ \\
\hline \multicolumn{5}{|l|}{ Tipulidae (CRANE FLIES) } \\
\hline Antocha & - & & 21 & $1.70 \%$ \\
\hline
\end{tabular}


Table 5. Benthic-macroinvertebrate data-Continued

01472170 (Site 1) - Pickering Creek near Eagle, Pa.

\begin{tabular}{|c|c|c|c|c|}
\hline Date & \multicolumn{2}{|c|}{ 10/12/95 } & \multicolumn{2}{|c|}{ 10/31/96 } \\
\hline Total count & \multicolumn{2}{|c|}{533} & \multicolumn{2}{|c|}{757} \\
\hline Total number of taxa & \multicolumn{2}{|c|}{23} & \multicolumn{2}{|c|}{28} \\
\hline Organism & Count & Percent & Count & Percent \\
\hline \multicolumn{5}{|l|}{ Platyhelminthes } \\
\hline \multicolumn{5}{|l|}{ Turbellaria (FLATWORMS) } \\
\hline \multicolumn{5}{|l|}{ Tricladida } \\
\hline Planariidae & 4 & $0.75 \%$ & 1 & $0.13 \%$ \\
\hline \multicolumn{5}{|l|}{ Nemertea (PROBOSCIS WORMS) } \\
\hline \multicolumn{5}{|l|}{ Enopla } \\
\hline \multicolumn{5}{|l|}{ Hoplonemertea } \\
\hline \multicolumn{5}{|l|}{ Tetrastemmatidae } \\
\hline Prostoma & - & & 1 & $.13 \%$ \\
\hline \multicolumn{5}{|l|}{ Mollusca } \\
\hline \multicolumn{5}{|l|}{ Gastropoda (SNAILS) } \\
\hline \multicolumn{5}{|l|}{ Basommatophora } \\
\hline \multicolumn{5}{|l|}{ Ancylidae } \\
\hline Ferrissia & 2 & $.38 \%$ & - & \\
\hline \multicolumn{5}{|l|}{ Annelida (SEGMENTED WORMS) } \\
\hline \multicolumn{5}{|l|}{ Oligochaeta } \\
\hline \multicolumn{5}{|l|}{ Lumbriculida } \\
\hline Lumbriculidae & - & & 4 & $.53 \%$ \\
\hline \multicolumn{5}{|l|}{ Tubificida } \\
\hline Naididae & 3 & $.56 \%$ & - & \\
\hline Arthropoda & & & & \\
\hline Acariformes & & & & \\
\hline Hydrachnidia (WATER MITES) & 20 & $3.75 \%$ & 40 & $5.28 \%$ \\
\hline Crustacea & & & & \\
\hline Cyclopoida & - & & 1 & $.13 \%$ \\
\hline Insecta & & & & \\
\hline Ephemeroptera (MAYFLIES) & & & & \\
\hline Baetidae & & & & \\
\hline Pseudocloeon & - & & 4 & $.53 \%$ \\
\hline Ephemerellidae & & & & \\
\hline Ephemerella & 2 & $.38 \%$ & 8 & $1.06 \%$ \\
\hline Heptageniidae & & & & \\
\hline Epeorus & - & & 1 & $.13 \%$ \\
\hline Stenonema & 11 & $2.06 \%$ & 9 & $1.19 \%$ \\
\hline Isonychiidae & & & & \\
\hline Isonychia & 2 & $.38 \%$ & - & \\
\hline Plecoptera (STONEFLIES) & & & & \\
\hline Capniidae & & & & \\
\hline Allocapnia & - & & 3 & $.40 \%$ \\
\hline Taeniopterygidae & & & & \\
\hline Taeniopteryx & 8 & $1.50 \%$ & 18 & $2.38 \%$ \\
\hline Trichoptera (CADDISFLIES) & & & & \\
\hline Glossosomatidae & & & & \\
\hline Glossosoma & 1 & $.19 \%$ & 21 & $2.77 \%$ \\
\hline Hydropsychidae & & & & \\
\hline Ceratopsyche & 12 & $2.25 \%$ & 99 & $13.08 \%$ \\
\hline Cheumatopsyche & 5 & $.94 \%$ & 27 & $3.57 \%$ \\
\hline Hydropsyche & 10 & $1.88 \%$ & 51 & $6.74 \%$ \\
\hline
\end{tabular}


Table 5. Benthic-macroinvertebrate data-Continued

01472170 (Site 1) - Pickering Creek near Eagle, Pa.-Continued

\begin{tabular}{|c|c|c|c|c|}
\hline Date & \multicolumn{2}{|c|}{$10 / 12 / 95$} & \multicolumn{2}{|c|}{$10 / 31 / 96$} \\
\hline Total count & \multicolumn{2}{|c|}{533} & \multicolumn{2}{|c|}{757} \\
\hline Total number of taxa & \multicolumn{2}{|c|}{23} & \multicolumn{2}{|c|}{28} \\
\hline Organism & Count & Percent & Count & Percent \\
\hline \multicolumn{5}{|l|}{ Trichoptera (CADDISFLIES) } \\
\hline \multicolumn{5}{|l|}{ Hydroptilidae } \\
\hline Hydroptila & 12 & $2.25 \%$ & 12 & $1.59 \%$ \\
\hline Leucotrichia & 122 & $22.89 \%$ & 90 & $11.89 \%$ \\
\hline \multicolumn{5}{|l|}{ Philopotamidae } \\
\hline Chimarra & 19 & $3.56 \%$ & 1 & $.13 \%$ \\
\hline Dolophilodes & - & & 2 & $.26 \%$ \\
\hline \multicolumn{5}{|l|}{ Polycentropodidae } \\
\hline Neureclipsis & - & & 1 & $.13 \%$ \\
\hline \multicolumn{5}{|l|}{ Psychomyiidae } \\
\hline Psychomyia & 10 & $1.88 \%$ & 32 & $4.23 \%$ \\
\hline \multicolumn{5}{|l|}{ Coleoptera (BEETLES) } \\
\hline \multicolumn{5}{|l|}{ Elmidae (RIFFLE BEETLES) } \\
\hline Optioservus & 22 & $4.13 \%$ & 30 & $3.96 \%$ \\
\hline Oulimnius & 1 & $.19 \%$ & - & \\
\hline Stenelmis & - & & 2 & $.26 \%$ \\
\hline \multicolumn{5}{|l|}{ Psephenidae (WATER PENNIES) } \\
\hline Psephenus & 11 & $2.06 \%$ & 2 & $.26 \%$ \\
\hline \multicolumn{5}{|l|}{ Diptera (TRUE FLIES) } \\
\hline Chironomidae (MIDGES) & 212 & $39.77 \%$ & 135 & $17.83 \%$ \\
\hline \multicolumn{5}{|l|}{ Empididae (DANCE FLIES) } \\
\hline Hemerodromia & 1 & $.19 \%$ & 4 & $.53 \%$ \\
\hline \multicolumn{5}{|l|}{ Simuliidae (BLACK FLIES) } \\
\hline Simulium & 8 & $1.50 \%$ & 3 & $.40 \%$ \\
\hline \multicolumn{5}{|l|}{ Tipulidae (CRANE FLIES) } \\
\hline Antocha & 35 & $6.57 \%$ & 155 & $20.48 \%$ \\
\hline
\end{tabular}


Table 5. Benthic-macroinvertebrate data-Continued

01472174 (Site 2) - Pickering Creek near Chester Springs, Pa.

\begin{tabular}{|c|c|c|}
\hline Date & \multicolumn{2}{|c|}{ 10/12/95 } \\
\hline Total count & \multicolumn{2}{|c|}{223} \\
\hline Total number of taxa & \multicolumn{2}{|c|}{26} \\
\hline Organism & Count & Percent \\
\hline \multicolumn{3}{|l|}{ Platyhelminthes } \\
\hline \multicolumn{3}{|l|}{ Turbellaria (FLATWORMS) } \\
\hline \multicolumn{3}{|l|}{ Tricladida } \\
\hline Planariidae & 1 & $0.45 \%$ \\
\hline \multicolumn{3}{|l|}{ Mollusca } \\
\hline \multicolumn{3}{|l|}{ Gastropoda (SNAILS) } \\
\hline \multicolumn{3}{|l|}{ Basommatophora } \\
\hline \multicolumn{3}{|l|}{ Ancylidae } \\
\hline Ferrissia & 7 & $3.14 \%$ \\
\hline \multicolumn{3}{|l|}{ Annelida (SEGMENTED WORMS) } \\
\hline \multicolumn{3}{|l|}{ Oligochaeta } \\
\hline \multicolumn{3}{|l|}{ Tubificida } \\
\hline Naididae & 2 & $.90 \%$ \\
\hline \multicolumn{3}{|l|}{ Arthropoda } \\
\hline \multicolumn{3}{|l|}{ Acariformes } \\
\hline Hydrachnidia (WATER MITES) & 39 & $17.49 \%$ \\
\hline \multicolumn{3}{|l|}{ Insecta } \\
\hline \multicolumn{3}{|l|}{ Ephemeroptera (MAYFLIES) } \\
\hline \multicolumn{3}{|l|}{ Baetidae } \\
\hline Pseudocloeon & 1 & $.45 \%$ \\
\hline \multicolumn{3}{|l|}{ Ephemerellidae } \\
\hline Ephemerella & 2 & $.90 \%$ \\
\hline \multicolumn{3}{|l|}{ Heptageniidae } \\
\hline Stenonema & 20 & $8.97 \%$ \\
\hline \multicolumn{3}{|l|}{ Isonychiidae } \\
\hline Isonychia & 4 & $1.79 \%$ \\
\hline \multicolumn{3}{|l|}{ Odonata (DRAGONFLIES AND DAMSELFLIES) } \\
\hline \multicolumn{3}{|l|}{ Coenagrionidae } \\
\hline Argia & 1 & $.45 \%$ \\
\hline Gomphidae & 1 & $.45 \%$ \\
\hline Plecoptera (STONEFLIES) & & \\
\hline Capniidae & & \\
\hline Allocapnia & 6 & $2.69 \%$ \\
\hline Taeniopterygidae & & \\
\hline Taeniopteryx & 8 & $3.59 \%$ \\
\hline Trichoptera (CADDISFLIES) & & \\
\hline Apataniidae & & \\
\hline Apatania & 2 & $.90 \%$ \\
\hline Glossosomatidae & & \\
\hline Glossosoma & 1 & $.45 \%$ \\
\hline Hydropsychidae & & \\
\hline Ceratopsyche & 14 & $6.28 \%$ \\
\hline Cheumatopsyche & 19 & $8.52 \%$ \\
\hline Hydropsyche & 1 & $.45 \%$ \\
\hline Hydroptilidae & & \\
\hline Leucotrichia & 6 & $2.69 \%$ \\
\hline
\end{tabular}


Table 5. Benthic-macroinvertebrate data-Continued

01472174 (Site 2) - Pickering Creek near Chester Springs, Pa.-Continued

\begin{tabular}{|c|c|c|}
\hline Date & \multicolumn{2}{|c|}{ 10/12/95 } \\
\hline Total count & \multicolumn{2}{|c|}{223} \\
\hline Total number of taxa & \multicolumn{2}{|c|}{26} \\
\hline Organism & Count & Percent \\
\hline \multicolumn{3}{|l|}{ Trichoptera (CADDISFLIES) } \\
\hline \multicolumn{3}{|l|}{ Philopotamidae } \\
\hline Chimarra & 5 & $2.24 \%$ \\
\hline \multicolumn{3}{|l|}{ Psychomyiidae } \\
\hline Psychomyia & 4 & $1.79 \%$ \\
\hline \multicolumn{3}{|l|}{ Coleoptera (BEETLES) } \\
\hline \multicolumn{3}{|l|}{ Elmidae (RIFFLE BEETLES) } \\
\hline Optioservus & 46 & $20.63 \%$ \\
\hline Stenelmis & 4 & $1.79 \%$ \\
\hline \multicolumn{3}{|c|}{ Psephenidae (WATER PENNIES) } \\
\hline Psephenus & 1 & $.45 \%$ \\
\hline \multicolumn{3}{|l|}{ Diptera (TRUE FLIES) } \\
\hline Chironomidae (MIDGES) & 20 & $8.97 \%$ \\
\hline \multicolumn{3}{|l|}{ Empididae (DANCE FLIES) } \\
\hline Hemerodromia & 3 & $1.35 \%$ \\
\hline \multicolumn{3}{|l|}{ Tipulidae (CRANE FLIES) } \\
\hline Antocha & 5 & $2.24 \%$ \\
\hline
\end{tabular}


Table 5. Benthic-macroinvertebrate data-Continued

014721854 (Site 3) - Pickering Creek at Merlin, Pa

\begin{tabular}{|c|c|c|c|c|}
\hline Date & \multicolumn{2}{|c|}{$10 / 12 / 95$} & \multicolumn{2}{|c|}{$10 / 16 / 97$} \\
\hline Total count & \multicolumn{2}{|c|}{457} & \multicolumn{2}{|c|}{1,463} \\
\hline Total number of taxa & \multicolumn{2}{|c|}{30} & \multicolumn{2}{|c|}{41} \\
\hline Organism & Count & Percent & Count & Percent \\
\hline \multicolumn{5}{|l|}{ Platyhelminthes } \\
\hline \multicolumn{5}{|l|}{ Turbellaria (FLATWORMS) } \\
\hline \multicolumn{5}{|l|}{ Tricladida } \\
\hline Planariidae & - & & 4 & $0.27 \%$ \\
\hline \multicolumn{5}{|l|}{ Nemertea (PROBOSCIS WORMS) } \\
\hline \multicolumn{5}{|l|}{ Enopla } \\
\hline \multicolumn{5}{|l|}{ Hoplonemertea } \\
\hline \multicolumn{5}{|l|}{ Tetrastemmatidae } \\
\hline Prostoma & - & & 2 & $.14 \%$ \\
\hline \multicolumn{5}{|l|}{ Mollusca } \\
\hline \multicolumn{5}{|l|}{ Gastropoda (SNAILS) } \\
\hline \multicolumn{5}{|l|}{ Basommatophora } \\
\hline \multicolumn{5}{|l|}{ Ancylidae } \\
\hline Ferrissia & 14 & $3.06 \%$ & 17 & $1.16 \%$ \\
\hline \multicolumn{5}{|l|}{ Lymnaeidae } \\
\hline Lymnaea & - & & 2 & $.14 \%$ \\
\hline \multicolumn{5}{|l|}{ Annelida (SEGMENTED WORMS) } \\
\hline \multicolumn{5}{|l|}{ Oligochaeta } \\
\hline Tubificida & & & & \\
\hline Naididae & 2 & $.44 \%$ & 7 & $.48 \%$ \\
\hline Arthropoda & & & & \\
\hline Acariformes & & & & \\
\hline Hydrachnidia (WATER MITES) & 29 & $6.35 \%$ & 178 & $12.17 \%$ \\
\hline Crustacea & & & & \\
\hline Cyclopoida & - & & 1 & $.07 \%$ \\
\hline Insecta & & & & \\
\hline Ephemeroptera (MAYFLIES) & & & & \\
\hline Baetidae & & & & \\
\hline Pseudocloeon & - & & 2 & $.14 \%$ \\
\hline Ephemerellidae & & & & \\
\hline Ephemerella & 3 & $.66 \%$ & 7 & $.48 \%$ \\
\hline Heptageniidae & & & & \\
\hline Stenonema & 14 & $3.06 \%$ & 46 & $3.14 \%$ \\
\hline Isonychiidae & & & & \\
\hline Isonychia & 21 & $4.60 \%$ & 1 & $.07 \%$ \\
\hline Leptohyphidae & - & & 1 & $.07 \%$ \\
\hline Odonata (DRAGONFLIES AND DAMSELFLIES) & & & & \\
\hline Gomphidae & & & & \\
\hline Lanthus & - & & 1 & $.07 \%$ \\
\hline Plecoptera (STONEFLIES) & & & & \\
\hline Capniidae & & & & \\
\hline Allocapnia & - & & 17 & $1.16 \%$ \\
\hline Chloroperlidae & - & & 2 & $.14 \%$ \\
\hline Perlidae & & & & \\
\hline Acroneuria & - & & 2 & $.14 \%$ \\
\hline Taeniopterygidae & & & & \\
\hline Taeniopteryx & 11 & $2.41 \%$ & 80 & $5.47 \%$ \\
\hline Hemiptera (TRUE FLIES) & & & & \\
\hline Veliidae & & & & \\
\hline Rhagovelia & - & & 3 & $.21 \%$ \\
\hline
\end{tabular}


Table 5. Benthic-macroinvertebrate data-Continued

014721854 (Site 3) - Pickering Creek at Merlin, Pa —Continued

\begin{tabular}{|c|c|c|c|c|}
\hline Date & \multicolumn{2}{|c|}{ 10/12/95 } & \multicolumn{2}{|c|}{ 10/16/97 } \\
\hline Total count & \multicolumn{2}{|c|}{457} & \multicolumn{2}{|c|}{1,463} \\
\hline Total number of taxa & \multicolumn{2}{|c|}{30} & \multicolumn{2}{|c|}{41} \\
\hline Organism & Count & Percent & Count & Percent \\
\hline \multicolumn{5}{|l|}{ Trichoptera (CADDISFLIES) } \\
\hline \multicolumn{5}{|l|}{ Apataniidae } \\
\hline Apatania & 16 & $3.50 \%$ & 53 & $3.62 \%$ \\
\hline \multicolumn{5}{|l|}{ Brachycentridae } \\
\hline Micrasema & 38 & $8.32 \%$ & 84 & $5.74 \%$ \\
\hline \multicolumn{5}{|l|}{ Glossosomatidae } \\
\hline Glossosoma & - & & 3 & $.21 \%$ \\
\hline \multicolumn{5}{|l|}{ Goeridae } \\
\hline Goera & 2 & $.44 \%$ & - & \\
\hline \multicolumn{5}{|l|}{ Hydropsychidae } \\
\hline Ceratopsyche & 96 & $21.01 \%$ & 82 & $5.60 \%$ \\
\hline Cheumatopsyche & 31 & $6.78 \%$ & 52 & $3.55 \%$ \\
\hline Hydropsyche & 7 & $1.53 \%$ & 8 & $.55 \%$ \\
\hline \multicolumn{5}{|l|}{ Hydroptilidae } \\
\hline Hydroptila & 1 & $.22 \%$ & 20 & $1.37 \%$ \\
\hline Leucotrichia & 1 & $.22 \%$ & 1 & $.07 \%$ \\
\hline \multicolumn{5}{|l|}{ Leptoceridae } \\
\hline Mystacides & - & & 3 & $.21 \%$ \\
\hline Oecetis & 1 & $.22 \%$ & 6 & $.41 \%$ \\
\hline \multicolumn{5}{|l|}{ Philopotamidae } \\
\hline Dolophilodes & - & & 1 & $.07 \%$ \\
\hline \multicolumn{5}{|l|}{ Polycentropodidae } \\
\hline Nyctiophylax & 1 & $.22 \%$ & - & \\
\hline Polycentropus & - & & 2 & $.14 \%$ \\
\hline \multicolumn{5}{|l|}{ Psychomyiidae } \\
\hline Psychomyia & 6 & $1.31 \%$ & 8 & $.55 \%$ \\
\hline \multicolumn{5}{|l|}{ Lepidoptera } \\
\hline \multicolumn{5}{|l|}{ Pyralidae (MOTHS) } \\
\hline Petrophila & 1 & $.22 \%$ & 1 & $.07 \%$ \\
\hline \multicolumn{5}{|l|}{ Coleoptera (BEETLES) } \\
\hline Dytiscidae & 1 & $.22 \%$ & - & \\
\hline Elmidae (RIFFLE BEETLES) & & & & \\
\hline Ancyronyx & - & & 1 & $.07 \%$ \\
\hline Dubiraphia & 15 & $3.28 \%$ & 1 & $.07 \%$ \\
\hline Macronychus & 1 & $.22 \%$ & - & \\
\hline Optioservus & 13 & $2.84 \%$ & 17 & $1.16 \%$ \\
\hline Oulimnius & - & & 5 & $.34 \%$ \\
\hline Promoresia & 1 & $.22 \%$ & - & \\
\hline Stenelmis & 1 & $.22 \%$ & 3 & $.21 \%$ \\
\hline Psephenidae (WATER PENNI & & & & \\
\hline Psephenus & 3 & $.66 \%$ & - & \\
\hline Diptera (TRUE FLIES) & & & & \\
\hline Chironomidae (MIDGES) & 68 & $14.88 \%$ & 560 & $38.28 \%$ \\
\hline Empididae (DANCE FLIES) & & & & \\
\hline Hemerodromia & 2 & $.44 \%$ & 12 & $.82 \%$ \\
\hline Simuliidae (BLACK FLIES) & & & & \\
\hline Simulium & 7 & $1.53 \%$ & 7 & $.48 \%$ \\
\hline Tipulidae (CRANE FLIES) & & & & \\
\hline Antocha & 50 & $1.94 \%$ & 160 & $10.94 \%$ \\
\hline
\end{tabular}


Table 5. Benthic-macroinvertebrate data-Continued

014721884 (Site 4) - Pickering Creek at Charlestown Road at Charlestown, Pa.

\begin{tabular}{|c|c|c|c|c|}
\hline Date & \multicolumn{2}{|c|}{$10 / 11 / 95$} & \multicolumn{2}{|c|}{$10 / 02 / 96$} \\
\hline Total count & \multicolumn{2}{|c|}{811} & \multicolumn{2}{|c|}{1,340} \\
\hline Total number of taxa & \multicolumn{2}{|c|}{42} & \multicolumn{2}{|c|}{37} \\
\hline Organism & Count & Percent & Count & Percent \\
\hline \multicolumn{5}{|l|}{ Platyhelminthes } \\
\hline \multicolumn{5}{|l|}{ Turbellaria (FLATWORMS) } \\
\hline \multicolumn{5}{|l|}{ Tricladida } \\
\hline Planariidae & 5 & $0.62 \%$ & 5 & $0.37 \%$ \\
\hline Nematoda (NEMATODES) & 3 & $.37 \%$ & 1 & $.07 \%$ \\
\hline \multicolumn{5}{|l|}{ Nemertea (PROBOSCIS WORMS) } \\
\hline \multicolumn{5}{|l|}{ Enopla } \\
\hline \multicolumn{5}{|l|}{ Hoplonemertea } \\
\hline \multicolumn{5}{|l|}{ Tetrastemmatidae } \\
\hline Prostoma & 1 & $.12 \%$ & 2 & $.15 \%$ \\
\hline \multicolumn{5}{|l|}{ Mollusca } \\
\hline \multicolumn{5}{|l|}{ Gastropoda (SNAILS) } \\
\hline \multicolumn{5}{|l|}{ Basommatophora } \\
\hline \multicolumn{5}{|l|}{ Ancylidae } \\
\hline Ferrissia & 15 & $1.85 \%$ & 3 & $.22 \%$ \\
\hline Planorbidae & & & & \\
\hline Gyraulus & 5 & $.62 \%$ & - & \\
\hline Annelida (SEGMENTED WORMS) & & & & \\
\hline Oligochaeta & 1 & $.12 \%$ & - & \\
\hline Lumbriculida & & & & \\
\hline Lumbriculidae & - & & 1 & $.07 \%$ \\
\hline Tubificida & & & & \\
\hline Naididae & 5 & $.62 \%$ & 2 & $.15 \%$ \\
\hline Arthropoda & & & & \\
\hline Acariformes & & & & \\
\hline Hydrachnidia (WATER MITES) & 74 & $9.12 \%$ & 38 & $2.84 \%$ \\
\hline Crustacea & & & & \\
\hline Cyclopoida & 5 & $.62 \%$ & - & \\
\hline Isopoda (SOW BUGS) & & & & \\
\hline Asellidae & 1 & $.12 \%$ & - & \\
\hline Insecta & & & & \\
\hline Ephemeroptera (MAYFLIES) & & & & \\
\hline Baetidae & & & & \\
\hline Baetis & - & & 44 & $3.28 \%$ \\
\hline Pseudocloeon & - & & 24 & $1.79 \%$ \\
\hline Caenidae & & & & \\
\hline Caenis & 26 & $3.21 \%$ & - & \\
\hline Ephemerellidae & & & & \\
\hline Ephemerella & 21 & $2.59 \%$ & 11 & $.82 \%$ \\
\hline Heptageniidae & & & & \\
\hline Epeorus & - & & 1 & $.07 \%$ \\
\hline Stenonema & 17 & $2.10 \%$ & 22 & $1.64 \%$ \\
\hline Isonychiidae & & & & \\
\hline Isonychia & 4 & $.49 \%$ & 27 & $2.01 \%$ \\
\hline Odonata (DRAGONFLIES AND D & & & & \\
\hline Coenagrionidae & 1 & $.12 \%$ & - & \\
\hline Plecoptera (STONEFLIES) & & & & \\
\hline Nemouridae & - & & 1 & $.07 \%$ \\
\hline Perlidae & & & & \\
\hline Acroneuria & - & & 4 & $.30 \%$ \\
\hline Taeniopterygidae & & & & \\
\hline Taeniopteryx & 2 & $.25 \%$ & 7 & $.52 \%$ \\
\hline Megaloptera & & & & \\
\hline Corydalidae (FISHFLIES AN & & & & \\
\hline Nigronia & - & & 3 & $.22 \%$ \\
\hline
\end{tabular}


Table 5. Benthic-macroinvertebrate data-Continued

014721884 (Site 4) - Pickering Creek at Charlestown Road at Charlestown, Pa.-Continued

\begin{tabular}{|c|c|c|c|c|}
\hline Date & \multicolumn{2}{|c|}{$10 / 11 / 95$} & \multicolumn{2}{|c|}{$10 / 02 / 96$} \\
\hline Total count & \multicolumn{2}{|c|}{811} & \multicolumn{2}{|c|}{1,340} \\
\hline Total number of taxa & \multicolumn{2}{|c|}{42} & \multicolumn{2}{|c|}{37} \\
\hline Organism & Count & Percent & Count & Percent \\
\hline $\begin{array}{l}\text { Trichoptera (CADDISFLIES) } \\
\text { Apataniidae }\end{array}$ & & & & \\
\hline $\begin{array}{c}\text { Apatania } \\
\text { Brachycentridae }\end{array}$ & 31 & $3.82 \%$ & 30 & $2.24 \%$ \\
\hline $\begin{array}{c}\text { Micrasema } \\
\text { Glossosomatidae }\end{array}$ & 5 & $.62 \%$ & 37 & $2.76 \%$ \\
\hline $\begin{array}{l}\text { Glossosoma } \\
\text { Goeridae }\end{array}$ & - & & 2 & $.15 \%$ \\
\hline $\begin{array}{c}\text { Goera } \\
\text { Helicopsychidae }\end{array}$ & 1 & $.12 \%$ & 1 & $.07 \%$ \\
\hline $\begin{array}{l}\text { Helicopsyche } \\
\text { Hydropsychidae }\end{array}$ & 20 & $2.47 \%$ & - & \\
\hline Ceratopsyche & 65 & $8.01 \%$ & 304 & $22.69 \%$ \\
\hline Cheumatopsyche & 44 & $5.43 \%$ & 275 & $20.52 \%$ \\
\hline Hydropsyche & 7 & $.86 \%$ & 71 & $5.30 \%$ \\
\hline Macrostemum & - & & 2 & $.15 \%$ \\
\hline Hydroptilidae & & & & \\
\hline Hydroptila & 8 & $.99 \%$ & - & \\
\hline Leucotrichia & 41 & $5.06 \%$ & 94 & $7.01 \%$ \\
\hline Leptoceridae & & & & \\
\hline Mystacides & 12 & $1.48 \%$ & - & \\
\hline Oecetis & 2 & $.25 \%$ & - & \\
\hline Philopotamidae & & & & \\
\hline Chimarra & 1 & $.12 \%$ & 14 & $1.04 \%$ \\
\hline $\begin{array}{c}\text { Polycentropodidae } \\
\text { Neureclipsis }\end{array}$ & 1 & $.12 \%$ & - & \\
\hline Nyctiophylax & 1 & $.12 \%$ & 2 & $.15 \%$ \\
\hline Polycentropus & 6 & $.74 \%$ & - & \\
\hline Psychomyiidae & & & & \\
\hline Psychomyia & 117 & $14.43 \%$ & 23 & $1.72 \%$ \\
\hline Lepidoptera & & & & \\
\hline Pyralidae (MOTHS) & & & & \\
\hline Petrophila & 6 & $.74 \%$ & 3 & $.22 \%$ \\
\hline Coleoptera (BEETLES) & & & & \\
\hline Elmidae (RIFFLE BEETLES & & & & \\
\hline Ancyronyx & 1 & $.12 \%$ & - & \\
\hline Dubiraphia & 5 & $.62 \%$ & - & \\
\hline Oulimnius & 15 & $1.85 \%$ & 7 & $.52 \%$ \\
\hline Promoresia & 2 & $.25 \%$ & - & \\
\hline Stenelmis & 3 & $.37 \%$ & 5 & $.37 \%$ \\
\hline Psephenidae (WATER PENN & & & & \\
\hline Psephenus & 1 & $.12 \%$ & 6 & $.45 \%$ \\
\hline Diptera (TRUE FLIES) & & & & \\
\hline Chironomidae (MIDGES) & 198 & $24.41 \%$ & 180 & $13.43 \%$ \\
\hline Empididae (DANCE FLIES) & & & & \\
\hline Hemerodromia & 7 & $.86 \%$ & 4 & $.30 \%$ \\
\hline Simuliidae (BLACK FLIES) & & & & \\
\hline Simulium & - & & 55 & $4.10 \%$ \\
\hline Tipulidae (CRANE FLIES) & & & & \\
\hline Antocha & 25 & $3.08 \%$ & 29 & $2.16 \%$ \\
\hline
\end{tabular}


Table 5. Benthic-macroinvertebrate data-Continued

01472190 (Site 5) - Pickering Creek near Phoenixville, Pa.

\begin{tabular}{|c|c|c|c|c|c|c|}
\hline Date & \multicolumn{2}{|c|}{$10 / 11 / 95$} & \multicolumn{2}{|c|}{$10 / 31 / 96$} & \multicolumn{2}{|c|}{$10 / 30 / 97$} \\
\hline Total count & \multicolumn{2}{|c|}{872} & \multicolumn{2}{|c|}{418} & \multicolumn{2}{|c|}{1,530} \\
\hline Total number of taxa & \multicolumn{2}{|c|}{35} & \multicolumn{2}{|c|}{26} & \multicolumn{2}{|c|}{36} \\
\hline Organism & Count & Percent & Count & Percent & Count & Percent \\
\hline \multicolumn{7}{|l|}{ Platyhelminthes } \\
\hline \multicolumn{7}{|l|}{ Turbellaria (FLATWORMS) } \\
\hline \multicolumn{7}{|l|}{ Tricladida } \\
\hline Planariidae & - & & - & & 2 & $0.13 \%$ \\
\hline Nematoda (NEMATODES) & - & & 1 & $0.24 \%$ & - & \\
\hline \multicolumn{7}{|l|}{ Mollusca } \\
\hline \multicolumn{7}{|l|}{ Gastropoda (SNAILS) } \\
\hline \multicolumn{7}{|l|}{ Basommatophora } \\
\hline \multicolumn{7}{|l|}{ Ancylidae } \\
\hline Ferrissia & 3 & $0.34 \%$ & - & & 16 & $1.05 \%$ \\
\hline \multicolumn{7}{|l|}{ Planorbidae } \\
\hline Gyraulus & 1 & $.11 \%$ & - & & - & \\
\hline \multicolumn{7}{|l|}{ Annelida (SEGMENTED WORMS) } \\
\hline \multicolumn{7}{|l|}{ Oligochaeta } \\
\hline \multicolumn{7}{|l|}{ Lumbriculida } \\
\hline Lumbriculidae & - & & 1 & $.24 \%$ & 1 & $.07 \%$ \\
\hline \multicolumn{7}{|l|}{ Tubificida } \\
\hline Naididae & 1 & $.11 \%$ & 12 & $2.87 \%$ & - & \\
\hline Arthropoda & & & & & & \\
\hline Acariformes & & & & & & \\
\hline Hydrachnidia (WATER MITES) & 20 & $2.29 \%$ & 26 & $6.22 \%$ & 50 & $3.27 \%$ \\
\hline Crustacea & & & & & & \\
\hline Cyclopoida & - & & - & & 1 & $.07 \%$ \\
\hline Insecta & & & & & & \\
\hline Ephemeroptera (MAYFLIES) & & & & & & \\
\hline Baetidae & & & & & & \\
\hline Baetis & - & & 1 & $.24 \%$ & 1 & $.07 \%$ \\
\hline Pseudocloeon & 2 & $.23 \%$ & 2 & $.48 \%$ & - & \\
\hline Ephemerellidae & & & & & & \\
\hline Ephemerella & 47 & $5.39 \%$ & 23 & $5.50 \%$ & 75 & $4.90 \%$ \\
\hline Heptageniidae & & & & & & \\
\hline Epeorus & 3 & $.34 \%$ & - & & - & \\
\hline Stenonema & 23 & $2.64 \%$ & 4 & $.96 \%$ & 76 & $4.97 \%$ \\
\hline Isonychiidae & & & & & & \\
\hline Isonychia & 5 & $.57 \%$ & 3 & $.72 \%$ & 6 & $.39 \%$ \\
\hline Plecoptera (STONEFLIES) & & & & & & \\
\hline Capniidae & & & & & & \\
\hline Allocapnia & 5 & $.57 \%$ & 25 & $5.98 \%$ & 2 & $.13 \%$ \\
\hline Chloroperlidae & 2 & $.23 \%$ & 1 & $.24 \%$ & - & \\
\hline Perlidae & & & & & & \\
\hline Acroneuria & 5 & $.57 \%$ & 1 & $.24 \%$ & 12 & $.78 \%$ \\
\hline Agnetina & 1 & $.11 \%$ & - & & 1 & $.07 \%$ \\
\hline Paragnetina & 4 & $.46 \%$ & 1 & $.24 \%$ & 2 & $.13 \%$ \\
\hline Taeniopterygidae & & & & & & \\
\hline Strophopteryx & - & & 17 & $4.07 \%$ & - & \\
\hline Taeniopteryx & 9 & $1.03 \%$ & 10 & $2.39 \%$ & 15 & $.98 \%$ \\
\hline
\end{tabular}


Table 5. Benthic-macroinvertebrate data-Continued

01472190 (Site 5) - Pickering Creek near Phoenixville, Pa.-Continued

\begin{tabular}{|c|c|c|c|c|c|c|}
\hline Date & \multicolumn{2}{|c|}{$10 / 11 / 95$} & \multicolumn{2}{|c|}{$10 / 31 / 96$} & \multicolumn{2}{|c|}{$10 / 30 / 97$} \\
\hline Total count & \multicolumn{2}{|c|}{872} & \multicolumn{2}{|c|}{418} & \multicolumn{2}{|c|}{1,530} \\
\hline Total number of taxa & \multicolumn{2}{|c|}{35} & \multicolumn{2}{|c|}{26} & \multicolumn{2}{|c|}{36} \\
\hline Organism & Count & Percent & Count & Percent & Count & Percent \\
\hline \multicolumn{7}{|l|}{ Trichoptera (CADDISFLIES) } \\
\hline \multicolumn{7}{|l|}{ Apataniidae } \\
\hline Apatania & 4 & $0.46 \%$ & - & & 29 & $1.90 \%$ \\
\hline \multicolumn{7}{|l|}{ Brachycentridae } \\
\hline Micrasema & 12 & $1.38 \%$ & - & & 74 & $4.84 \%$ \\
\hline \multicolumn{7}{|l|}{ Glossosomatidae } \\
\hline Glossosoma & 2 & $.23 \%$ & 4 & $0.96 \%$ & 51 & $3.33 \%$ \\
\hline Protoptila & 5 & $.57 \%$ & - & & 4 & $.26 \%$ \\
\hline \multicolumn{7}{|l|}{ Helicopsychidae } \\
\hline Helicopsyche & - & & - & & 6 & $.39 \%$ \\
\hline \multicolumn{7}{|l|}{ Hydropsychidae } \\
\hline Ceratopsyche & 140 & $16.06 \%$ & 72 & $17.22 \%$ & 283 & $18.50 \%$ \\
\hline Cheumatopsyche & 37 & $4.24 \%$ & 9 & $2.15 \%$ & 63 & $4.12 \%$ \\
\hline Hydropsyche & 2 & $.23 \%$ & - & & 13 & $.85 \%$ \\
\hline Macrostemum & 5 & $.57 \%$ & - & & 8 & $.52 \%$ \\
\hline \multicolumn{7}{|l|}{ Hydroptilidae } \\
\hline Leucotrichia & 211 & $24.20 \%$ & 2 & $.48 \%$ & 372 & $24.31 \%$ \\
\hline \multicolumn{7}{|l|}{ Lepidostomatidae } \\
\hline Lepidostoma & - & & - & & 2 & $.13 \%$ \\
\hline \multicolumn{7}{|l|}{ Philopotamidae } \\
\hline Chimarra & 5 & $.57 \%$ & 1 & $.24 \%$ & 18 & $1.18 \%$ \\
\hline \multicolumn{7}{|l|}{ Polycentropodidae } \\
\hline Nyctiophylax & 1 & $.11 \%$ & - & & - & \\
\hline Polycentropus & 1 & $.11 \%$ & - & & - & \\
\hline \multicolumn{7}{|l|}{ Psychomyiidae } \\
\hline $\begin{array}{l}\text { Psychomyia } \\
\text { Uenoidae }\end{array}$ & 20 & $2.29 \%$ & 1 & $.24 \%$ & 80 & $5.23 \%$ \\
\hline Neophylax & - & & - & & 6 & $.39 \%$ \\
\hline Lepidoptera & & & & & & \\
\hline Pyralidae (MOTHS) & & & & & & \\
\hline Petrophila & - & & 1 & $.24 \%$ & 1 & $.07 \%$ \\
\hline Coleoptera (BEETLES) & & & & & & \\
\hline Elmidae (RIFFLE BEETLES & & & & & & \\
\hline Optioservus & 2 & $.23 \%$ & 3 & $.72 \%$ & 19 & $1.24 \%$ \\
\hline Oulimnius & - & & - & & 2 & $.13 \%$ \\
\hline Promoresia & 1 & $.11 \%$ & - & & - & \\
\hline Stenelmis & 2 & $.23 \%$ & - & & 7 & $.46 \%$ \\
\hline Psephenidae & & & & & & \\
\hline Psephenus & - & & - & & 3 & $.20 \%$ \\
\hline Diptera (TRUE FLIES) & & & & & & \\
\hline Chironomidae (MIDGES) & 236 & $27.06 \%$ & 167 & $39.95 \%$ & 140 & $9.15 \%$ \\
\hline Empididae (DANCE FLIES) & & & & & & \\
\hline Hemerodromia & 1 & $.11 \%$ & - & & 9 & $.59 \%$ \\
\hline Simuliidae (BLACK FLIES) & & & & & & \\
\hline Simulium & 4 & $.46 \%$ & 15 & $3.59 \%$ & - & \\
\hline Tipulidae (CRANE FLIES) & & & & & & \\
\hline Antocha & 50 & $5.73 \%$ & 15 & $3.59 \%$ & 80 & $5.23 \%$ \\
\hline
\end{tabular}


Table 5. Benthic-macroinvertebrate data-Continued

01473167 (Site 49) - Little Valley Creek at Howellville, Pa.

\begin{tabular}{|c|c|c|c|c|}
\hline Date & \multicolumn{2}{|c|}{ 10/30/95 } & \multicolumn{2}{|c|}{$10 / 24 / 96$} \\
\hline Total count & \multicolumn{2}{|c|}{395} & \multicolumn{2}{|c|}{113} \\
\hline Total number of taxa & \multicolumn{2}{|c|}{22} & \multicolumn{2}{|c|}{16} \\
\hline Organism & Count & Percent & Count & Percent \\
\hline \multicolumn{5}{|l|}{ Platyhelminthes } \\
\hline \multicolumn{5}{|l|}{ Turbellaria (FLATWORMS) } \\
\hline \multicolumn{5}{|l|}{ Tricladida } \\
\hline Planariidae & 20 & $5.06 \%$ & 1 & $0.88 \%$ \\
\hline \multicolumn{5}{|l|}{ Annelida (SEGMENTED WORMS) } \\
\hline \multicolumn{5}{|l|}{ Polychaeta } \\
\hline \multicolumn{5}{|l|}{ Sabellida } \\
\hline \multicolumn{5}{|l|}{ Sabellidae } \\
\hline Manayunkia speciosa & 48 & $12.15 \%$ & - & \\
\hline \multicolumn{5}{|l|}{ Oligochaeta } \\
\hline \multicolumn{5}{|l|}{ Tubificida } \\
\hline Naididae & 1 & $.25 \%$ & - & \\
\hline \multicolumn{5}{|l|}{ Arthropoda } \\
\hline \multicolumn{5}{|l|}{ Acariformes } \\
\hline Hydrachnidia (WATER MITES) & 2 & $.51 \%$ & 5 & $4.42 \%$ \\
\hline \multicolumn{5}{|l|}{ Crustacea } \\
\hline \multicolumn{5}{|l|}{ Amphipoda (SCUDS) } \\
\hline \multicolumn{5}{|l|}{ Gammaridae } \\
\hline Gammarus & - & & 2 & $1.77 \%$ \\
\hline \multicolumn{5}{|l|}{ Isopoda (SOW BUGS) } \\
\hline Asellidae & & & & \\
\hline Caecidotea & - & & 1 & $.88 \%$ \\
\hline Insecta & & & & \\
\hline Ephemeroptera (MAYFLIES) & & & & \\
\hline Baetidae & & & & \\
\hline Baetis & 2 & $.51 \%$ & 13 & $11.50 \%$ \\
\hline Pseudocloeon & 6 & $1.52 \%$ & 1 & $.88 \%$ \\
\hline Ephemerellidae & & & & \\
\hline Ephemerella & 71 & $17.97 \%$ & 10 & $8.85 \%$ \\
\hline Heptageniidae & & & & \\
\hline Stenonema & - & & 1 & $.88 \%$ \\
\hline Plecoptera (STONEFLIES) & & & & \\
\hline Capniidae & & & & \\
\hline Allocapnia & 4 & $1.01 \%$ & 5 & $4.42 \%$ \\
\hline Taeniopterygidae & & & & \\
\hline Taeniopteryx & 1 & $.25 \%$ & - & \\
\hline Trichoptera (CADDISFLIES) & & & & \\
\hline Glossosomatidae & & & & \\
\hline Glossosoma & 3 & $.76 \%$ & - & \\
\hline Hydropsychidae & & & & \\
\hline Ceratopsyche & 42 & $10.63 \%$ & 11 & $9.73 \%$ \\
\hline Cheumatopsyche & 5 & $1.27 \%$ & 1 & $.88 \%$ \\
\hline Hydropsyche & 17 & $4.30 \%$ & - & \\
\hline Philopotamidae & & & & \\
\hline Dolophilodes & - & & 1 & $.88 \%$ \\
\hline Psychomyiidae & & & & \\
\hline Psychomyia & 1 & $.25 \%$ & - & \\
\hline
\end{tabular}


Table 5. Benthic-macroinvertebrate data-Continued

01473167 (Site 49) - Little Valley Creek at Howellville, Pa.-Continued

\begin{tabular}{|c|c|c|c|c|}
\hline Date & \multicolumn{2}{|c|}{$10 / 30 / 95$} & \multicolumn{2}{|c|}{$10 / 24 / 96$} \\
\hline Total count & \multicolumn{2}{|c|}{395} & \multicolumn{2}{|c|}{113} \\
\hline Total number of taxa & \multicolumn{2}{|c|}{22} & \multicolumn{2}{|c|}{16} \\
\hline Organism & Count & Percent & Count & Percent \\
\hline \multicolumn{5}{|l|}{ Coleoptera (BEETLES) } \\
\hline \multicolumn{4}{|l|}{ Elmidae (RIFFLE BEETLES) } & \\
\hline Optioservus & 74 & $18.73 \%$ & 28 & $24.78 \%$ \\
\hline Oulimnius & 4 & $1.01 \%$ & - & \\
\hline \multicolumn{5}{|c|}{ Psephenidae (WATER PENNIES) } \\
\hline Ectopria & 1 & $.25 \%$ & - & \\
\hline Diptera (TRUE FLIES) & 1 & $.25 \%$ & - & \\
\hline Chironomidae (MIDGES) & 62 & $15.70 \%$ & 20 & $17.70 \%$ \\
\hline \multicolumn{5}{|l|}{ Empididae (DANCE FLIES) } \\
\hline Hemerodromia & 1 & $.25 \%$ & - & \\
\hline \multicolumn{5}{|l|}{ Simuliidae (BLACK FLIES) } \\
\hline Simulium & - & & 6 & $5.31 \%$ \\
\hline \multicolumn{5}{|l|}{ Tipulidae (CRANE FLIES) } \\
\hline Antocha & 28 & $7.09 \%$ & 7 & $6.19 \%$ \\
\hline
\end{tabular}


Table 5. Benthic-macroinvertebrate data-Continued

01473168 (Site 50) - Valley Creek near Valley Forge, Pa.

\begin{tabular}{|c|c|c|c|c|}
\hline Date & \multicolumn{2}{|c|}{$10 / 30 / 95$} & \multicolumn{2}{|c|}{$10 / 24 / 96$} \\
\hline Total count & \multicolumn{2}{|c|}{1,032} & \multicolumn{2}{|c|}{870} \\
\hline Total number of taxa & \multicolumn{2}{|c|}{18} & \multicolumn{2}{|c|}{23} \\
\hline Organism & Count & Percent & Count & Percent \\
\hline \multicolumn{5}{|l|}{ Platyhelminthes } \\
\hline \multicolumn{5}{|l|}{ Turbellaria (FLATWORMS) } \\
\hline \multicolumn{5}{|l|}{ Tricladida } \\
\hline Planariidae & 60 & $5.81 \%$ & 104 & $11.95 \%$ \\
\hline Nematoda (NEMATODES) & 1 & $.10 \%$ & - & \\
\hline \multicolumn{5}{|l|}{ Annelida (SEGMENTED WORMS) } \\
\hline Oligochaeta & - & & 2 & $.23 \%$ \\
\hline \multicolumn{5}{|l|}{ Tubificida } \\
\hline Naididae & 5 & $.48 \%$ & 5 & $.57 \%$ \\
\hline \multicolumn{5}{|l|}{ Arthropoda } \\
\hline \multicolumn{5}{|l|}{ Acariformes } \\
\hline Hydrachnidia (WATER MITES) & 1 & $.10 \%$ & 9 & $1.03 \%$ \\
\hline \multicolumn{5}{|l|}{ Crustacea } \\
\hline \multicolumn{5}{|l|}{ Amphipoda (SCUDS) } \\
\hline \multicolumn{5}{|l|}{ Gammaridae } \\
\hline Gammarus & 21 & $2.03 \%$ & 9 & $1.03 \%$ \\
\hline \multicolumn{5}{|l|}{ Isopoda (SOW BUGS) } \\
\hline \multicolumn{5}{|l|}{ Asellidae } \\
\hline Lirceus & - & & 1 & $.11 \%$ \\
\hline \multicolumn{5}{|l|}{ Insecta } \\
\hline \multicolumn{5}{|l|}{ Ephemeroptera (MAYFLIES) } \\
\hline \multicolumn{5}{|l|}{ Baetidae } \\
\hline Baetis & - & & 2 & $.23 \%$ \\
\hline Pseudocloeon & - & & 1 & $.11 \%$ \\
\hline Ephemerellidae & & & & \\
\hline Ephemerella & 6 & $.58 \%$ & 13 & $1.49 \%$ \\
\hline Plecoptera (STONEFLIES) & & & & \\
\hline Taeniopterygidae & & & & \\
\hline Taeniopteryx & - & & 1 & $.11 \%$ \\
\hline Trichoptera (CADDISFLIES) & & & & \\
\hline Glossosomatidae & & & & \\
\hline Glossosoma & - & & 2 & $.23 \%$ \\
\hline Hydropsychidae & & & & \\
\hline Ceratopsyche & 184 & $17.83 \%$ & 62 & $7.13 \%$ \\
\hline Cheumatopsyche & 24 & $2.33 \%$ & - & \\
\hline Hydropsyche & 230 & $22.29 \%$ & 44 & $5.06 \%$ \\
\hline Philopotamidae & & & & \\
\hline Chimarra & 30 & $2.91 \%$ & 2 & $.23 \%$ \\
\hline Coleoptera (BEETLES) & & & & \\
\hline Elmidae (RIFFLE BEETLES & & & & \\
\hline Dubiraphia & 1 & $.10 \%$ & - & \\
\hline Optioservus & 162 & $15.70 \%$ & 249 & $28.62 \%$ \\
\hline Oulimnius & - & & 14 & $1.61 \%$ \\
\hline Stenelmis & 12 & $1.16 \%$ & 20 & $2.30 \%$ \\
\hline Psephenidae (WATER PENN & & & & \\
\hline Ectopria & 1 & $.10 \%$ & - & \\
\hline Psephenus & - & & 3 & $.34 \%$ \\
\hline
\end{tabular}


Table 5. Benthic-macroinvertebrate data-Continued

01473168 (Site 50) - Valley Creek near Valley Forge, Pa.-Continued

\begin{tabular}{|c|c|c|c|c|}
\hline Date & \multicolumn{2}{|c|}{$10 / 30 / 95$} & \multicolumn{2}{|c|}{$10 / 24 / 96$} \\
\hline Total count & \multicolumn{2}{|c|}{1,032} & \multicolumn{2}{|c|}{870} \\
\hline Total number of taxa & \multicolumn{2}{|c|}{18} & \multicolumn{2}{|c|}{23} \\
\hline Organism & Count & Percent & Count & Percent \\
\hline Diptera (TRUE FLIES) & & & & \\
\hline $\begin{array}{l}\text { Chironomidae (MIDGES) } \\
\text { Empididae (DANCE FLIES) }\end{array}$ & 217 & $21.03 \%$ & 211 & $24.25 \%$ \\
\hline $\begin{array}{c}\text { Hemerodromia } \\
\text { Simuliidae (BLACK FLIES) }\end{array}$ & 4 & $.39 \%$ & 2 & $.23 \%$ \\
\hline $\begin{array}{c}\text { Simulium } \\
\text { Tipulidae (CRANE FLIES) }\end{array}$ & 11 & $1.07 \%$ & 11 & $1.26 \%$ \\
\hline Antocha & 62 & $6.01 \%$ & 101 & $11.61 \%$ \\
\hline Tipula & - & & 2 & $.23 \%$ \\
\hline
\end{tabular}


Table 5. Benthic-macroinvertebrate data-Continued

01475300 (Site 17) - Darby Creek at Waterloo Mills near Devon, Pa.

\begin{tabular}{|c|c|c|c|c|}
\hline Date & \multicolumn{2}{|c|}{$10 / 19 / 95$} & \multicolumn{2}{|c|}{$10 / 16 / 96$} \\
\hline Total count & \multicolumn{2}{|c|}{463} & \multicolumn{2}{|c|}{256} \\
\hline Total number of taxa & \multicolumn{2}{|c|}{25} & \multicolumn{2}{|c|}{20} \\
\hline Organism & Count & Percent & Count & Percent \\
\hline \multicolumn{5}{|l|}{ Platyhelminthes } \\
\hline \multicolumn{5}{|l|}{ Turbellaria (FLATWORMS) } \\
\hline \multicolumn{5}{|l|}{ Tricladida } \\
\hline Planariidae & 3 & $0.65 \%$ & 15 & $5.86 \%$ \\
\hline \multicolumn{5}{|l|}{ Nemertea (PROBOSCIS WORMS) } \\
\hline \multicolumn{5}{|l|}{ Enopla } \\
\hline \multicolumn{5}{|l|}{ Hoplonemertea } \\
\hline \multicolumn{5}{|l|}{ Tetrastemmatidae } \\
\hline Prostoma & 1 & $.22 \%$ & - & \\
\hline \multicolumn{5}{|l|}{ Mollusca } \\
\hline \multicolumn{5}{|l|}{ Gastropoda (SNAILS) } \\
\hline \multicolumn{5}{|l|}{ Basommatophora } \\
\hline \multicolumn{5}{|l|}{ Ancylidae } \\
\hline Ferrissia & 2 & $.43 \%$ & 1 & $.39 \%$ \\
\hline \multicolumn{5}{|l|}{ Arthropoda } \\
\hline \multicolumn{5}{|l|}{ Acariformes } \\
\hline Hydrachnidia (WATER MITES) & 28 & $6.05 \%$ & 6 & $2.34 \%$ \\
\hline Crustacea & & & & \\
\hline Cladocera & 5 & $1.08 \%$ & - & \\
\hline Cyclopoida & 322 & $69.55 \%$ & - & \\
\hline Amphipoda (SCUDS) & & & & \\
\hline Gammaridae & & & & \\
\hline Gammarus & 8 & $1.73 \%$ & 1 & $.39 \%$ \\
\hline Insecta & & & & \\
\hline Ephemeroptera (MAYFLIES) & & & & \\
\hline Caenidae & & & & \\
\hline Caenis & 2 & $.43 \%$ & - & \\
\hline Ephemerellidae & & & & \\
\hline Ephemerella & 3 & $.65 \%$ & 1 & $.39 \%$ \\
\hline Heptageniidae & & & & \\
\hline Stenonema & 1 & $.22 \%$ & 6 & $2.34 \%$ \\
\hline Odonata (DRAGONFLIES AND & & & & \\
\hline Coenagrionidae & & & & \\
\hline Argia & 1 & $.22 \%$ & - & \\
\hline Plecoptera (STONEFLIES) & & & & \\
\hline Capniidae & & & & \\
\hline Allocapnia & - & & 1 & $.39 \%$ \\
\hline Trichoptera (CADDISFLIES) & & & & \\
\hline Glossosomatidae & & & & \\
\hline Glossosoma & - & & 2 & $.78 \%$ \\
\hline Hydropsychidae & & & & \\
\hline Ceratopsyche & 7 & $1.51 \%$ & 11 & $4.30 \%$ \\
\hline Cheumatopsyche & 6 & $1.30 \%$ & 6 & $2.34 \%$ \\
\hline Hydropsyche & 4 & $.86 \%$ & 13 & $5.08 \%$ \\
\hline Hydroptilidae & & & & \\
\hline Hydroptila & 1 & $.22 \%$ & 4 & $1.56 \%$ \\
\hline Leptoceridae & & & & \\
\hline Oecetis & - & & 1 & $.39 \%$ \\
\hline Philopotamidae & & & & \\
\hline Chimarra & 1 & $.22 \%$ & 10 & $3.91 \%$ \\
\hline Psychomyiidae & & & & \\
\hline Psychomyia & 1 & $.22 \%$ & - & \\
\hline
\end{tabular}


Table 5. Benthic-macroinvertebrate data-Continued

01475300 (Site 17) - Darby Creek at Waterloo Mills near Devon, Pa.-Continued

\begin{tabular}{|c|c|c|c|c|}
\hline Date & \multicolumn{2}{|c|}{$10 / 19 / 95$} & \multicolumn{2}{|c|}{$10 / 16 / 96$} \\
\hline Total count & \multicolumn{2}{|c|}{463} & \multicolumn{2}{|c|}{256} \\
\hline Total number of taxa & \multicolumn{2}{|c|}{25} & \multicolumn{2}{|c|}{20} \\
\hline Organism & Count & Percent & Count & Percent \\
\hline \multicolumn{5}{|l|}{ Coleoptera (BEETLES) } \\
\hline \multicolumn{5}{|l|}{ Elmidae (RIFFLE BEETLES) } \\
\hline Optioservus & 5 & $1.08 \%$ & 9 & $3.52 \%$ \\
\hline Oulimnius & 1 & $.22 \%$ & - & \\
\hline Stenelmis & 5 & $1.08 \%$ & 11 & $4.30 \%$ \\
\hline \multicolumn{5}{|c|}{ Psephenidae (WATER PENNIES) } \\
\hline Ectopria & 1 & $.22 \%$ & - & \\
\hline Psephenus & 16 & $3.46 \%$ & 10 & $3.91 \%$ \\
\hline \multicolumn{5}{|l|}{ Diptera (TRUE FLIES) } \\
\hline Chironomidae (MIDGES) & 30 & $6.48 \%$ & 80 & $31.25 \%$ \\
\hline \multicolumn{5}{|l|}{ Empididae (DANCE FLIES) } \\
\hline Hemerodromia & 1 & $.22 \%$ & 4 & $1.56 \%$ \\
\hline \multicolumn{5}{|l|}{ Tipulidae (CRANE FLIES) } \\
\hline Antocha & 8 & $1.73 \%$ & 64 & $25.00 \%$ \\
\hline
\end{tabular}


Table 5. Benthic-macroinvertebrate data-Continued

01475840 (Site 19) - Crum Creek at Whitehorse, Pa.

\begin{tabular}{|c|c|c|c|c|}
\hline Date & \multicolumn{2}{|c|}{ 10/19/95 } & \multicolumn{2}{|c|}{ 10/16/96 } \\
\hline Total count & \multicolumn{2}{|c|}{603} & \multicolumn{2}{|c|}{886} \\
\hline Total number of taxa & \multicolumn{2}{|c|}{33} & \multicolumn{2}{|c|}{29} \\
\hline Organism & Count & Percent & Count & Percent \\
\hline \multicolumn{5}{|l|}{ Platyhelminthes } \\
\hline \multicolumn{5}{|l|}{ Turbellaria (FLATWORMS) } \\
\hline \multicolumn{5}{|l|}{ Tricladida } \\
\hline Planariidae & 12 & $1.99 \%$ & 12 & $1.35 \%$ \\
\hline Nematoda (NEMATODES) & 3 & $.50 \%$ & - & \\
\hline \multicolumn{5}{|l|}{ Nemertea (PROBOSCIS WORMS) } \\
\hline \multicolumn{5}{|l|}{ Enopla } \\
\hline \multicolumn{5}{|l|}{ Hoplonemertea } \\
\hline \multicolumn{5}{|l|}{ Tetrastemmatidae } \\
\hline Prostoma & - & & 4 & $.45 \%$ \\
\hline \multicolumn{5}{|l|}{ Mollusca } \\
\hline \multicolumn{5}{|l|}{ Gastropoda (SNAILS) } \\
\hline \multicolumn{5}{|l|}{ Basommatophora } \\
\hline \multicolumn{5}{|l|}{ Ancylidae } \\
\hline Ferrissia & 1 & $.17 \%$ & 1 & $.11 \%$ \\
\hline \multicolumn{5}{|l|}{ Annelida (SEGMENTED WORMS) } \\
\hline \multicolumn{5}{|l|}{ Oligochaeta } \\
\hline \multicolumn{5}{|l|}{ Tubificida } \\
\hline Naididae & 50 & $8.29 \%$ & 8 & $.90 \%$ \\
\hline \multicolumn{5}{|l|}{ Arthropoda } \\
\hline Acariformes & & & & \\
\hline Hydrachnidia (WATER MITES) & 43 & $7.13 \%$ & 42 & $4.74 \%$ \\
\hline Crustacea & & & & \\
\hline Cyclopoida & 10 & $1.66 \%$ & - & \\
\hline Insecta & & & & \\
\hline Ephemeroptera (MAYFLIES) & & & & \\
\hline Baetidae & & & & \\
\hline Baetis & - & & 6 & $.68 \%$ \\
\hline Pseudocloeon & 1 & $.17 \%$ & 4 & $.45 \%$ \\
\hline Caenidae & & & & \\
\hline Caenis & 2 & $.33 \%$ & - & \\
\hline Ephemerellidae & & & & \\
\hline Ephemerella & 14 & $2.32 \%$ & 4 & $.45 \%$ \\
\hline Heptageniidae & & & & \\
\hline Stenonema & 5 & $.83 \%$ & 18 & $2.03 \%$ \\
\hline Isonychiidae & & & & \\
\hline Isonychia & 1 & $.17 \%$ & 8 & $.90 \%$ \\
\hline Plecoptera (STONEFLIES) & & & & \\
\hline Capniidae & & & & \\
\hline Allocapnia & 2 & $.33 \%$ & 1 & $.11 \%$ \\
\hline Perlidae & & & & \\
\hline Acroneuria & 1 & $.17 \%$ & - & \\
\hline Taeniopterygidae & & & & \\
\hline Taeniopteryx & 9 & $1.49 \%$ & 11 & $1.24 \%$ \\
\hline Megaloptera & & & & \\
\hline Corydalidae (FISHFLIES AN & & & & \\
\hline Corydalus & 1 & $.17 \%$ & 1 & $.11 \%$ \\
\hline
\end{tabular}


Table 5. Benthic-macroinvertebrate data-Continued

01475840 (Site 19) - Crum Creek at Whitehorse, Pa.-Continued

\begin{tabular}{|c|c|c|c|c|}
\hline Date & \multicolumn{2}{|c|}{ 10/19/95 } & \multicolumn{2}{|c|}{$10 / 16 / 96$} \\
\hline Total count & \multicolumn{2}{|c|}{603} & \multicolumn{2}{|c|}{886} \\
\hline Total number of taxa & \multicolumn{2}{|c|}{33} & \multicolumn{2}{|c|}{29} \\
\hline Organism & Count & Percent & Count & Percent \\
\hline \multicolumn{5}{|l|}{ Trichoptera (CADDISFLIES) } \\
\hline \multicolumn{5}{|l|}{ Glossosomatidae } \\
\hline Glossosoma & 1 & $0.17 \%$ & 1 & $0.11 \%$ \\
\hline \multicolumn{5}{|l|}{ Hydropsychidae } \\
\hline Ceratopsyche & 60 & $9.95 \%$ & 54 & $6.09 \%$ \\
\hline Cheumatopsyche & 14 & $2.32 \%$ & 66 & $7.45 \%$ \\
\hline Hydropsyche & 5 & $.83 \%$ & 5 & $.56 \%$ \\
\hline \multicolumn{5}{|l|}{ Hydroptilidae } \\
\hline Hydroptila & 3 & $.50 \%$ & 7 & $.79 \%$ \\
\hline Leucotrichia & 4 & $.66 \%$ & 1 & $.11 \%$ \\
\hline \multicolumn{5}{|l|}{ Philopotamidae } \\
\hline Wormaldia & - & & 1 & $.11 \%$ \\
\hline \multicolumn{5}{|l|}{ Psychomyiidae } \\
\hline Psychomyia & 1 & $.17 \%$ & - & \\
\hline \multicolumn{5}{|l|}{ Lepidoptera } \\
\hline \multicolumn{5}{|l|}{ Pyralidae (MOTHS) } \\
\hline Petrophila & 14 & $2.32 \%$ & 2 & $.23 \%$ \\
\hline \multicolumn{5}{|l|}{ Coleoptera (BEETLES) } \\
\hline Curculionidae & 1 & $.17 \%$ & - & \\
\hline \multicolumn{5}{|l|}{ Elmidae (RIFFLE BEETLES) } \\
\hline Optioservus & 12 & $1.99 \%$ & 5 & $.56 \%$ \\
\hline Oulimnius & 4 & $.66 \%$ & - & \\
\hline Stenelmis & 3 & $.50 \%$ & 3 & $.34 \%$ \\
\hline \multicolumn{5}{|l|}{ Hydrophilidae } \\
\hline Berosus & - & & 1 & $.11 \%$ \\
\hline \multicolumn{5}{|l|}{ Psephenidae (WATER PENNIES) } \\
\hline Ectopria & 1 & $.17 \%$ & - & \\
\hline Psephenus & 4 & $.66 \%$ & 3 & $.34 \%$ \\
\hline \multicolumn{5}{|l|}{ Diptera (TRUE FLIES) } \\
\hline Chironomidae (MIDGES) & 300 & $49.75 \%$ & 430 & $48.53 \%$ \\
\hline \multicolumn{5}{|l|}{ Empididae (DANCE FLIES) } \\
\hline Hemerodromia & 9 & $1.49 \%$ & 19 & $2.14 \%$ \\
\hline \multicolumn{5}{|l|}{ Simuliidae (BLACK FLIES) } \\
\hline Simulium & 6 & $1.00 \%$ & 58 & $6.55 \%$ \\
\hline \multicolumn{5}{|l|}{ Tipulidae (CRANE FLIES) } \\
\hline Antocha & 6 & $1.00 \%$ & 110 & $12.42 \%$ \\
\hline
\end{tabular}


Table 5. Benthic-macroinvertebrate data-Continued

01476430 (Site 20) - Ridley Creek at Goshenville, Pa.

\begin{tabular}{|c|c|c|c|c|}
\hline Date & \multicolumn{2}{|c|}{ 10/25/95 } & \multicolumn{2}{|c|}{ 10/28/96 } \\
\hline Total count & \multicolumn{2}{|c|}{412} & \multicolumn{2}{|c|}{201} \\
\hline Total number of taxa & \multicolumn{2}{|c|}{25} & \multicolumn{2}{|c|}{19} \\
\hline Organism & Count & Percent & Count & Percent \\
\hline \multicolumn{5}{|l|}{ Platyhelminthes } \\
\hline \multicolumn{5}{|l|}{ Turbellaria (FLATWORMS) } \\
\hline \multicolumn{5}{|l|}{ Tricladida } \\
\hline Planariidae & 15 & $3.64 \%$ & 2 & $1.00 \%$ \\
\hline \multicolumn{5}{|l|}{ Mollusca } \\
\hline \multicolumn{5}{|l|}{ Gastropoda (SNAILS) } \\
\hline \multicolumn{5}{|l|}{ Basommatophora } \\
\hline \multicolumn{5}{|l|}{ Ancylidae } \\
\hline Ferrissia & 6 & $1.46 \%$ & 3 & $1.49 \%$ \\
\hline \multicolumn{5}{|l|}{ Planorbidae } \\
\hline Gyraulus & 2 & $.49 \%$ & - & \\
\hline \multicolumn{5}{|l|}{ Bivalvia (CLAMS) } \\
\hline \multicolumn{5}{|l|}{ Veneroida } \\
\hline Sphaeriidae & - & & 1 & $.50 \%$ \\
\hline \multicolumn{5}{|l|}{ Annelida (SEGMENTED WORMS) } \\
\hline \multicolumn{5}{|l|}{ Oligochaeta } \\
\hline \multicolumn{5}{|l|}{ Tubificida } \\
\hline Naididae & 2 & $.49 \%$ & 3 & $1.49 \%$ \\
\hline Arthropoda (ARTHROPODS) & & & & \\
\hline Acariformes & & & & \\
\hline Hydrachnidia (WATER MITES) & 39 & $9.47 \%$ & 4 & $1.99 \%$ \\
\hline Crustacea & & & & \\
\hline Isopoda (SOW BUGS) & & & & \\
\hline Asellidae & - & & 1 & $.50 \%$ \\
\hline Insecta & & & & \\
\hline Ephemeroptera (MAYFLIES) & & & & \\
\hline Caenidae & & & & \\
\hline Caenis & 1 & $.24 \%$ & - & \\
\hline Ephemerellidae & & & & \\
\hline Ephemerella & 30 & $7.28 \%$ & 9 & $4.48 \%$ \\
\hline Heptageniidae & & & & \\
\hline Stenonema & 35 & $8.50 \%$ & 5 & $2.49 \%$ \\
\hline Isonychiidae & & & & \\
\hline Isonychia & 1 & $.24 \%$ & - & \\
\hline Leptohyphidae & & & & \\
\hline Tricorythodes & 1 & $.24 \%$ & - & \\
\hline Plecoptera (STONEFLIES) & & & & \\
\hline Capniidae & & & & \\
\hline Allocapnia & - & & 1 & $.50 \%$ \\
\hline Trichoptera (CADDISFLIES) & & & & \\
\hline Hydropsychidae & & & & \\
\hline Ceratopsyche & 4 & $.97 \%$ & 23 & $11.44 \%$ \\
\hline Cheumatopsyche & 17 & $4.13 \%$ & 8 & $3.98 \%$ \\
\hline Hydropsyche & 20 & $4.85 \%$ & 13 & $6.47 \%$ \\
\hline Hydroptilidae & & & & \\
\hline Hydroptila & 6 & $1.46 \%$ & 2 & $1.00 \%$ \\
\hline Leucotrichia & 1 & $.24 \%$ & - & \\
\hline Leptoceridae & & & & \\
\hline Mystacides & 4 & $.97 \%$ & - & \\
\hline Philopotamidae & & & & \\
\hline Chimarra & 11 & $2.67 \%$ & - & \\
\hline
\end{tabular}


Table 5. Benthic-macroinvertebrate data-Continued

01476430 (Site 20) - Ridley Creek at Goshenville, Pa.-Continued

\begin{tabular}{|c|c|c|c|c|}
\hline Date & \multicolumn{2}{|c|}{$10 / 25 / 95$} & \multicolumn{2}{|c|}{$10 / 28 / 96$} \\
\hline Total count & \multicolumn{2}{|c|}{412} & \multicolumn{2}{|c|}{201} \\
\hline Total number of taxa & \multicolumn{2}{|c|}{25} & \multicolumn{2}{|c|}{19} \\
\hline Organism & Count & Percent & Count & Percent \\
\hline \multicolumn{5}{|l|}{ Coleoptera (BEETLES) } \\
\hline \multicolumn{5}{|l|}{ Elmidae (RIFFLE BEETLES) } \\
\hline Ancyronyx & 2 & $0.49 \%$ & - & \\
\hline Optioservus & 53 & $12.86 \%$ & 13 & $6.47 \%$ \\
\hline Oulimnius & 3 & $.73 \%$ & - & \\
\hline Stenelmis & 30 & $7.28 \%$ & 2 & $1.00 \%$ \\
\hline \multicolumn{5}{|c|}{ Psephenidae (WATER PENNIES) } \\
\hline Psephenus & 4 & $.97 \%$ & 7 & $3.48 \%$ \\
\hline \multicolumn{5}{|l|}{ Diptera (TRUE FLIES) } \\
\hline Chironomidae (MIDGES) & 43 & $10.44 \%$ & 29 & $14.43 \%$ \\
\hline \multicolumn{5}{|l|}{ Empididae (DANCE FLIES) } \\
\hline Hemerodromia & 1 & $.24 \%$ & - & \\
\hline \multicolumn{5}{|l|}{ Simuliidae (BLACK FLIES) } \\
\hline Simulium & - & & 58 & $28.86 \%$ \\
\hline \multicolumn{5}{|l|}{ Tipulidae (CRANE FLIES) } \\
\hline Antocha & 81 & $19.66 \%$ & 17 & $8.46 \%$ \\
\hline
\end{tabular}


Table 5. Benthic-macroinvertebrate data-Continued

01476435 (Site 21) - Ridley Creek at Dutton Mill near West Chester, Pa.

\begin{tabular}{|c|c|c|c|c|}
\hline Date & \multicolumn{2}{|c|}{$10 / 23 / 95$} & \multicolumn{2}{|c|}{$10 / 23 / 96$} \\
\hline Total count & \multicolumn{2}{|c|}{915} & \multicolumn{2}{|c|}{633} \\
\hline Total number of taxa & \multicolumn{2}{|c|}{36} & \multicolumn{2}{|c|}{34} \\
\hline Organism & Count & Percent & Count & Percent \\
\hline \multicolumn{5}{|l|}{ Platyhelminthes } \\
\hline \multicolumn{5}{|l|}{ Turbellaria (FLATWORMS) } \\
\hline \multicolumn{5}{|l|}{ Tricladida } \\
\hline Planariidae & 6 & $0.66 \%$ & 4 & $0.63 \%$ \\
\hline \multicolumn{5}{|l|}{ Nemertea (PROBOSCIS WORMS) } \\
\hline \multicolumn{5}{|l|}{ Enopla } \\
\hline \multicolumn{5}{|l|}{ Hoplonemertea } \\
\hline \multicolumn{5}{|l|}{ Tetrastemmatidae } \\
\hline Prostoma & - & & 4 & $.63 \%$ \\
\hline \multicolumn{5}{|l|}{ Mollusca } \\
\hline \multicolumn{5}{|l|}{ Gastropoda (SNAILS) } \\
\hline \multicolumn{5}{|l|}{ Basommatophora } \\
\hline \multicolumn{5}{|l|}{ Ancylidae } \\
\hline Ferrissia & - & & 5 & $.79 \%$ \\
\hline \multicolumn{5}{|l|}{ Bivalvia (CLAMS) } \\
\hline Veneroida & & & & \\
\hline Sphaeriidae & 2 & $.22 \%$ & - & \\
\hline Annelida (SEGMENTED WORMS) & & & & \\
\hline Oligochaeta & & & & \\
\hline Tubificida & & & & \\
\hline Naididae & 1 & $.11 \%$ & - & \\
\hline Tubificidae & 1 & $.11 \%$ & - & \\
\hline Arthropoda & & & & \\
\hline Acariformes & & & & \\
\hline Hydrachnidia (WATER MITES) & 25 & $2.73 \%$ & 43 & $6.79 \%$ \\
\hline Crustacea & & & & \\
\hline Isopoda (SOW BUGS) & & & & \\
\hline Asellidae & & & & \\
\hline Caecidotea & 1 & $.11 \%$ & - & \\
\hline Decapoda & & & & \\
\hline Cambaridae (CRAYFISH) & 1 & $.11 \%$ & 1 & $.16 \%$ \\
\hline Insecta & & & & \\
\hline Ephemeroptera (MAYFLIES) & & & & \\
\hline Baetidae & & & & \\
\hline Baetis & 3 & $.33 \%$ & 1 & $.16 \%$ \\
\hline Caenidae & & & & \\
\hline Caenis & 1 & $.11 \%$ & - & \\
\hline Ephemerellidae & & & & \\
\hline Ephemerella & 30 & $3.28 \%$ & 21 & $3.32 \%$ \\
\hline Heptageniidae & & & & \\
\hline Stenonema & 61 & $6.67 \%$ & 24 & $3.79 \%$ \\
\hline Isonychiidae & & & & \\
\hline Isonychia & 11 & $1.20 \%$ & 1 & $.16 \%$ \\
\hline Leptohyphidae & & & & \\
\hline Tricorythodes & 2 & $.22 \%$ & 1 & $.16 \%$ \\
\hline Plecoptera (STONEFLIES) & & & & \\
\hline Capniidae & & & & \\
\hline Allocapnia & 4 & $.44 \%$ & 3 & $.47 \%$ \\
\hline Taeniopterygidae & & & & \\
\hline Taeniopteryx & 13 & $1.42 \%$ & 10 & $1.58 \%$ \\
\hline Megaloptera & & & & \\
\hline Corydalidae (FISHFLIES AND DOBSONFLIES) & & & & \\
\hline Corydalus & 1 & $.11 \%$ & 1 & $.16 \%$ \\
\hline Trichoptera (CADDISFLIES) & & & & \\
\hline
\end{tabular}


Table 5. Benthic-macroinvertebrate data-Continued

01476435 (Site 21) - Ridley Creek at Dutton Mill near West Chester, Pa.-Continued

\begin{tabular}{|c|c|c|c|c|}
\hline Date & \multicolumn{2}{|c|}{$10 / 23 / 95$} & \multicolumn{2}{|c|}{$10 / 23 / 96$} \\
\hline Total count & \multicolumn{2}{|c|}{915} & \multicolumn{2}{|c|}{633} \\
\hline Total number of taxa & \multicolumn{2}{|c|}{36} & \multicolumn{2}{|c|}{34} \\
\hline Organism & Count & Percent & Count & Percent \\
\hline \multicolumn{5}{|l|}{ Brachycentridae } \\
\hline Micrasema & 3 & $0.33 \%$ & - & \\
\hline \multicolumn{5}{|l|}{ Glossosomatidae } \\
\hline Glossosoma & - & & 1 & $0.16 \%$ \\
\hline \multicolumn{5}{|l|}{ Hydropsychidae } \\
\hline Ceratopsyche & 135 & $14.75 \%$ & 108 & $17.06 \%$ \\
\hline Cheumatopsyche & 163 & $17.81 \%$ & 89 & $14.06 \%$ \\
\hline Hydropsyche & 5 & $.55 \%$ & 10 & $1.58 \%$ \\
\hline \multicolumn{5}{|l|}{ Hydroptilidae } \\
\hline Hydroptila & 26 & $2.84 \%$ & 7 & $1.11 \%$ \\
\hline Leucotrichia & 40 & $4.37 \%$ & 45 & $7.11 \%$ \\
\hline \multicolumn{5}{|l|}{ Leptoceridae } \\
\hline Mystacides & - & & 1 & $.16 \%$ \\
\hline \multicolumn{5}{|l|}{ Philopotamidae } \\
\hline Chimarra & 26 & $2.84 \%$ & - & \\
\hline Dolophilodes & 4 & $.44 \%$ & 1 & $.16 \%$ \\
\hline \multicolumn{5}{|l|}{ Uenoidae } \\
\hline Neophylax & - & & 1 & $.16 \%$ \\
\hline \multicolumn{5}{|l|}{ Lepidoptera } \\
\hline \multicolumn{5}{|l|}{ Pyralidae (MOTHS) } \\
\hline Petrophila & 7 & $.77 \%$ & 2 & $.32 \%$ \\
\hline \multicolumn{5}{|l|}{ Coleoptera (BEETLES) } \\
\hline \multicolumn{5}{|l|}{ Elmidae (RIFFLE BEETLES) } \\
\hline Ancyronyx & 2 & $.22 \%$ & - & \\
\hline Dubiraphia & 1 & $.11 \%$ & - & \\
\hline Macronychus & 2 & $.22 \%$ & 2 & $.32 \%$ \\
\hline Optioservus & 21 & $2.30 \%$ & 7 & $1.11 \%$ \\
\hline Oulimnius & 1 & $.11 \%$ & 1 & $.16 \%$ \\
\hline Stenelmis & 5 & $.55 \%$ & 1 & $.16 \%$ \\
\hline \multicolumn{5}{|c|}{ Psephenidae (WATER PENNIES) } \\
\hline Ectopria & - & & 1 & $.16 \%$ \\
\hline Psephenus & 16 & $1.75 \%$ & 10 & $1.58 \%$ \\
\hline \multicolumn{5}{|l|}{ Diptera (TRUE FLIES) } \\
\hline Chironomidae (MIDGES) & 220 & $24.04 \%$ & 134 & $21.17 \%$ \\
\hline \multicolumn{5}{|l|}{ Empididae (DANCE FLIES) } \\
\hline Chelifera & - & & 1 & $.16 \%$ \\
\hline Hemerodromia & 1 & $.11 \%$ & 2 & $.32 \%$ \\
\hline \multicolumn{5}{|l|}{ Simuliidae (BLACK FLIES) } \\
\hline Simulium & 10 & $1.09 \%$ & 15 & $2.37 \%$ \\
\hline \multicolumn{5}{|l|}{ Tipulidae (CRANE FLIES) } \\
\hline Antocha & 64 & $6.99 \%$ & 75 & $11.85 \%$ \\
\hline
\end{tabular}


Table 5. Benthic-macroinvertebrate data-Continued

01476790 (Site 22) - East Branch Chester Creek at Green Hill, Pa.

\begin{tabular}{|c|c|c|c|c|}
\hline Date & \multicolumn{2}{|c|}{ 10/23/95 } & \multicolumn{2}{|c|}{ 10/03/97 } \\
\hline Total count & \multicolumn{2}{|c|}{331} & \multicolumn{2}{|c|}{250} \\
\hline Total number of taxa & \multicolumn{2}{|c|}{22} & \multicolumn{2}{|c|}{17} \\
\hline Organism & Count & Percent & Count & Percent \\
\hline \multicolumn{5}{|l|}{ Platyhelminthes } \\
\hline \multicolumn{5}{|l|}{ Turbellaria (FLATWORMS) } \\
\hline \multicolumn{5}{|l|}{ Tricladida } \\
\hline Planariidae & 1 & $0.30 \%$ & 1 & $0.40 \%$ \\
\hline \multicolumn{5}{|l|}{ Mollusca } \\
\hline \multicolumn{5}{|l|}{ Gastropoda (SNAILS) } \\
\hline \multicolumn{5}{|l|}{ Basommatophora } \\
\hline \multicolumn{5}{|l|}{ Ancylidae } \\
\hline Ferrissia & 1 & $.30 \%$ & - & \\
\hline \multicolumn{5}{|l|}{ Physidae } \\
\hline Physa & 6 & $1.81 \%$ & - & \\
\hline \multicolumn{5}{|l|}{ Annelida (SEGMENTED WORMS) } \\
\hline \multicolumn{5}{|l|}{ Oligochaeta } \\
\hline \multicolumn{5}{|l|}{ Tubificida } \\
\hline Naididae & 3 & $.91 \%$ & - & \\
\hline \multicolumn{5}{|l|}{ Arthropoda } \\
\hline \multicolumn{5}{|l|}{ Acariformes } \\
\hline Hydrachnidia (WATER MITES) & 8 & $2.42 \%$ & 17 & $6.80 \%$ \\
\hline \multicolumn{5}{|l|}{ Crustacea } \\
\hline \multicolumn{5}{|l|}{ Isopoda (SOW BUGS) } \\
\hline \multicolumn{5}{|l|}{ Asellidae } \\
\hline Caecidotea & - & & 1 & $.40 \%$ \\
\hline \multicolumn{5}{|l|}{ Insecta } \\
\hline \multicolumn{5}{|l|}{ Ephemeroptera (MAYFLIES) } \\
\hline \multicolumn{5}{|l|}{ Baetidae } \\
\hline Baetis & - & & 14 & $5.60 \%$ \\
\hline Pseudocloeon & 2 & $.60 \%$ & 20 & $8.00 \%$ \\
\hline Ephemerellidae & & & & \\
\hline Ephemerella & 2 & $.60 \%$ & 2 & $.80 \%$ \\
\hline Heptageniidae & & & & \\
\hline Stenonema & 6 & $1.81 \%$ & 9 & $3.60 \%$ \\
\hline Odonata (DRAGONFLIES AND & & & & \\
\hline Coenagrionidae & & & & \\
\hline Argia & - & & 1 & $.40 \%$ \\
\hline Plecoptera (STONEFLIES) & & & & \\
\hline Capniidae & & & & \\
\hline Allocapnia & 1 & $.30 \%$ & - & \\
\hline Hemiptera (TRUE BUGS) & & & & \\
\hline Veliidae & & & & \\
\hline Rhagovelia & - & & 1 & $.40 \%$ \\
\hline
\end{tabular}


Table 5. Benthic-macroinvertebrate data-Continued

01476790 (Site 22) - East Branch Chester Creek at Green Hill, Pa.-Continued

\begin{tabular}{|c|c|c|c|c|}
\hline Date & \multicolumn{2}{|c|}{$10 / 23 / 95$} & \multicolumn{2}{|c|}{$10 / 03 / 97$} \\
\hline Total count & \multicolumn{2}{|c|}{331} & \multicolumn{2}{|c|}{250} \\
\hline Total number of taxa & \multicolumn{2}{|c|}{22} & \multicolumn{2}{|c|}{17} \\
\hline Organism & Count & Percent & Count & Percent \\
\hline \multicolumn{5}{|l|}{ Trichoptera (CADDISFLIES) } \\
\hline \multicolumn{5}{|l|}{ Glossosomatidae } \\
\hline Glossosoma & 36 & $10.88 \%$ & 19 & $7.60 \%$ \\
\hline \multicolumn{5}{|l|}{ Hydropsychidae } \\
\hline Ceratopsyche & 88 & $26.59 \%$ & 59 & $23.60 \%$ \\
\hline Cheumatopsyche & 19 & $5.74 \%$ & 15 & $6.00 \%$ \\
\hline Hydropsyche & 97 & $29.31 \%$ & 34 & $13.60 \%$ \\
\hline \multicolumn{5}{|l|}{ Leptoceridae } \\
\hline Mystacides & 2 & $.60 \%$ & - & \\
\hline \multicolumn{5}{|l|}{ Philopotamidae } \\
\hline Wormaldia & 1 & $.30 \%$ & 8 & $3.20 \%$ \\
\hline \multicolumn{5}{|l|}{ Psychomyiidae } \\
\hline Psychomyia & 1 & $.30 \%$ & - & \\
\hline \multicolumn{5}{|l|}{ Coleoptera (BEETLES) } \\
\hline \multicolumn{5}{|l|}{ Elmidae (RIFFLE BEETLES) } \\
\hline Optioservus & 2 & $.60 \%$ & 2 & $.80 \%$ \\
\hline \multicolumn{5}{|l|}{ Diptera (TRUE FLIES) } \\
\hline Chironomidae (MIDGES) & 44 & $13.29 \%$ & 40 & $16.00 \%$ \\
\hline \multicolumn{5}{|l|}{ Empididae (DANCE FLIES) } \\
\hline Hemerodromia & 2 & $.60 \%$ & - & \\
\hline \multicolumn{5}{|l|}{ Simuliidae (BLACK FLIES) } \\
\hline Simulium & 1 & $.30 \%$ & - & \\
\hline \multicolumn{5}{|l|}{ Tipulidae (CRANE FLIES) } \\
\hline Antocha & 7 & $2.11 \%$ & 7 & $2.80 \%$ \\
\hline Dicranota & 1 & $.30 \%$ & - & \\
\hline
\end{tabular}


Table 5. Benthic-macroinvertebrate data-Continued

01476830 (Site 23) - East Branch Chester Creek at Milltown, Pa.

\begin{tabular}{|c|c|c|c|c|}
\hline Date & \multicolumn{2}{|c|}{$10 / 24 / 95$} & \multicolumn{2}{|c|}{ 10/07/96 } \\
\hline Total count & \multicolumn{2}{|c|}{281} & \multicolumn{2}{|c|}{1,594} \\
\hline Total number of taxa & \multicolumn{2}{|c|}{25} & \multicolumn{2}{|c|}{29} \\
\hline Organism & Count & Percent & Count & Percent \\
\hline \multicolumn{5}{|l|}{ Platyhelminthes } \\
\hline \multicolumn{5}{|l|}{ Turbellaria (FLATWORMS) } \\
\hline \multicolumn{5}{|l|}{ Tricladida } \\
\hline Planariidae & 2 & $0.71 \%$ & 26 & $1.63 \%$ \\
\hline Nematoda (NEMATODES) & - & & 13 & $.82 \%$ \\
\hline \multicolumn{5}{|l|}{ Mollusca } \\
\hline \multicolumn{5}{|l|}{ Gastropoda (SNAILS) } \\
\hline \multicolumn{5}{|l|}{ Basommatophora } \\
\hline \multicolumn{5}{|l|}{ Ancylidae } \\
\hline Ferrissia & 1 & $.36 \%$ & 9 & $.56 \%$ \\
\hline \multicolumn{5}{|l|}{ Annelida (SEGMENTED WORMS) } \\
\hline \multicolumn{5}{|l|}{ Oligochaeta } \\
\hline \multicolumn{5}{|l|}{ Tubificida } \\
\hline Naididae & 7 & $2.49 \%$ & 200 & $12.55 \%$ \\
\hline \multicolumn{5}{|l|}{ Arthropoda } \\
\hline \multicolumn{5}{|l|}{ Acariformes } \\
\hline Hydrachnidia (WATER MITES) & 26 & $9.25 \%$ & 34 & $2.13 \%$ \\
\hline \multicolumn{5}{|l|}{ Crustacea } \\
\hline Cladocera & 5 & $1.78 \%$ & - & \\
\hline Cyclopoida & 19 & $6.76 \%$ & - & \\
\hline Podocopa (SEED SHRIMP) & - & & 1 & $.06 \%$ \\
\hline \multicolumn{5}{|l|}{ Insecta } \\
\hline \multicolumn{5}{|l|}{ Ephemeroptera (MAYFLIES) } \\
\hline \multicolumn{5}{|l|}{ Baetidae } \\
\hline Baetis & - & & 2 & $.13 \%$ \\
\hline \multicolumn{5}{|l|}{ Caenidae } \\
\hline Caenis & 1 & $.36 \%$ & - & \\
\hline \multicolumn{5}{|l|}{ Ephemerellidae } \\
\hline Ephemerella & 1 & $.36 \%$ & 4 & $.25 \%$ \\
\hline \multicolumn{5}{|l|}{ Heptageniidae } \\
\hline Stenonema & 1 & $.36 \%$ & 110 & $6.90 \%$ \\
\hline \multicolumn{5}{|l|}{ Leptohyphidae } \\
\hline Tricorythodes & 1 & $.36 \%$ & 3 & $.19 \%$ \\
\hline Odonata (DRAGONFLIES AND I & & & & \\
\hline Coenagrionidae & & & & \\
\hline Argia & - & & 1 & $.06 \%$ \\
\hline Hemiptera (TRUE BUGS) & & & & \\
\hline Corixidae & 1 & $.36 \%$ & - & \\
\hline
\end{tabular}


Table 5. Benthic-macroinvertebrate data-Continued

01476830 (Site 23) - East Branch Chester Creek at Milltown, Pa.-Continued

\begin{tabular}{|c|c|c|c|c|}
\hline Date & \multicolumn{2}{|c|}{$10 / 24 / 95$} & \multicolumn{2}{|c|}{$10 / 07 / 96$} \\
\hline Total count & \multicolumn{2}{|c|}{281} & \multicolumn{2}{|c|}{1,594} \\
\hline Total number of taxa & \multicolumn{2}{|c|}{25} & \multicolumn{2}{|c|}{29} \\
\hline Organism & Count & Percent & Count & Percent \\
\hline \multicolumn{5}{|l|}{ Trichoptera (CADDISFLIES) } \\
\hline \multicolumn{5}{|l|}{ Hydropsychidae } \\
\hline Ceratopsyche & - & & 43 & $2.70 \%$ \\
\hline Cheumatopsyche & 2 & $0.71 \%$ & 59 & $3.70 \%$ \\
\hline Hydropsyche & 1 & $.36 \%$ & 83 & $5.21 \%$ \\
\hline \multicolumn{5}{|l|}{ Hydroptilidae } \\
\hline Hydroptila & 75 & $26.69 \%$ & 135 & $8.47 \%$ \\
\hline \multicolumn{5}{|l|}{ Leptoceridae } \\
\hline Oecetis & 2 & $.71 \%$ & 42 & $2.63 \%$ \\
\hline \multicolumn{5}{|l|}{ Philopotamidae } \\
\hline Chimarra & 1 & $.36 \%$ & 1 & $.06 \%$ \\
\hline \multicolumn{5}{|l|}{ Rhyacophilidae } \\
\hline Rhyacophila & 1 & $.36 \%$ & - & \\
\hline \multicolumn{5}{|l|}{ Coleoptera (BEETLES) } \\
\hline \multicolumn{5}{|l|}{ Elmidae (RIFFLE BEETLES) } \\
\hline Ancyronyx & - & & 1 & $.06 \%$ \\
\hline Dubiraphia & - & & 3 & $.19 \%$ \\
\hline Microcylloepus & - & & 11 & $.69 \%$ \\
\hline Optioservus & 8 & $2.85 \%$ & 83 & $5.21 \%$ \\
\hline Oulimnius & 2 & $.71 \%$ & 2 & $.13 \%$ \\
\hline Stenelmis & 30 & $1.68 \%$ & 159 & $9.97 \%$ \\
\hline \multicolumn{5}{|c|}{ Psephenidae (WATER PENNIES) } \\
\hline Ectopria & - & & 1 & $.06 \%$ \\
\hline Psephenus & 26 & $9.25 \%$ & 44 & $2.76 \%$ \\
\hline \multicolumn{5}{|l|}{ Diptera (TRUE FLIES) } \\
\hline Chironomidae (MIDGES) & 56 & $19.93 \%$ & 375 & $23.53 \%$ \\
\hline \multicolumn{5}{|l|}{ Empididae (DANCE FLIES) } \\
\hline Hemerodromia & 4 & $1.42 \%$ & 110 & $6.90 \%$ \\
\hline \multicolumn{5}{|l|}{ Simuliidae (BLACK FLIES) } \\
\hline Simulium & 7 & $2.49 \%$ & 35 & $2.20 \%$ \\
\hline \multicolumn{5}{|l|}{ Tipulidae (CRANE FLIES) } \\
\hline Antocha & 1 & $.36 \%$ & 4 & $.25 \%$ \\
\hline
\end{tabular}


Table 5. Benthic-macroinvertebrate data-Continued

01476835 (Site 24) - East Branch Chester Creek at Westtown, Pa.

\begin{tabular}{|c|c|c|c|c|c|c|}
\hline Date & \multicolumn{2}{|c|}{$10 / 24 / 95$} & \multicolumn{2}{|c|}{$10 / 07 / 96$} & \multicolumn{2}{|c|}{$10 / 06 / 97$} \\
\hline Total count & \multicolumn{2}{|c|}{464} & \multicolumn{2}{|c|}{1,386} & \multicolumn{2}{|c|}{1,291} \\
\hline Total number of taxa & \multicolumn{2}{|c|}{26} & \multicolumn{2}{|c|}{30} & \multicolumn{2}{|c|}{26} \\
\hline Organism & Count & Percent & Count & Percent & Count & Percent \\
\hline \multicolumn{7}{|l|}{ Platyhelminthes } \\
\hline \multicolumn{7}{|l|}{ Turbellaria (FLATWORMS) } \\
\hline \multicolumn{7}{|l|}{ Tricladida } \\
\hline Planariidae & 14 & $3.02 \%$ & 15 & $1.08 \%$ & 26 & $2.01 \%$ \\
\hline \multicolumn{7}{|l|}{ Nemertea (PROBOSCIS WORMS) } \\
\hline \multicolumn{7}{|l|}{ Enopla } \\
\hline \multicolumn{7}{|l|}{ Hoplonemertea } \\
\hline \multicolumn{7}{|l|}{ Tetrastemmatidae } \\
\hline Prostoma & - & & 3 & $.22 \%$ & - & \\
\hline \multicolumn{7}{|l|}{ Mollusca } \\
\hline \multicolumn{7}{|l|}{ Gastropoda (SNAILS) } \\
\hline \multicolumn{7}{|l|}{ Basommatophora } \\
\hline \multicolumn{7}{|l|}{ Ancylidae } \\
\hline Ferrissia & - & & 2 & $.14 \%$ & - & \\
\hline \multicolumn{7}{|l|}{ Annelida (SEGMENTED WORMS) } \\
\hline \multicolumn{7}{|l|}{ Oligochaeta } \\
\hline Tubificida & & & & & & \\
\hline Naididae & 18 & $3.88 \%$ & 44 & $3.17 \%$ & - & \\
\hline Tubificidae & - & & - & & 2 & $.15 \%$ \\
\hline Arthropoda & & & & & & \\
\hline Acariformes & & & & & & \\
\hline Hydrachnidia (WATER MITES) & 12 & $2.59 \%$ & 31 & $2.24 \%$ & 20 & $1.55 \%$ \\
\hline Crustacea & & & & & & \\
\hline Cyclopoida & 11 & $2.37 \%$ & - & & - & \\
\hline Amphipoda (SCUDS) & & & & & & \\
\hline Crangonyctidae & & & & & & \\
\hline Crangonyx & - & & - & & 3 & $.23 \%$ \\
\hline Gammaridae & & & & & & \\
\hline Gammarus & - & & 6 & $.43 \%$ & - & \\
\hline Insecta & & & & & & \\
\hline Ephemeroptera (MAYFLIES) & & & & & & \\
\hline Baetidae & & & & & & \\
\hline Baetis & - & & 35 & $2.53 \%$ & 27 & $2.09 \%$ \\
\hline Pseudocloeon & - & & - & & 25 & $1.94 \%$ \\
\hline Ephemerellidae & & & & & & \\
\hline Ephemerella & 2 & $.43 \%$ & 7 & $.51 \%$ & - & \\
\hline Heptageniidae & & & & & & \\
\hline Stenonema & 4 & $.86 \%$ & 76 & $5.48 \%$ & 14 & $1.08 \%$ \\
\hline Odonata (DRAGONFLIES AND & & & & & & \\
\hline Coenagrionidae & & & & & & \\
\hline Argia & 1 & $.22 \%$ & - & & - & \\
\hline Plecoptera (STONEFLIES) & & & & & & \\
\hline Taeniopterygidae & & & & & & \\
\hline Taeniopteryx & - & & 1 & $.07 \%$ & - & \\
\hline
\end{tabular}


Table 5. Benthic-macroinvertebrate data-Continued

01476835 (Site 24) - East Branch Chester Creek at Westtown, Pa.-Continued

\begin{tabular}{|c|c|c|c|c|c|c|}
\hline Date & \multicolumn{2}{|c|}{ 10/24/95 } & \multicolumn{2}{|c|}{$10 / 07 / 96$} & \multicolumn{2}{|c|}{$10 / 06 / 97$} \\
\hline Total count & \multicolumn{2}{|c|}{464} & \multicolumn{2}{|c|}{1,386} & \multicolumn{2}{|c|}{1,291} \\
\hline Total number of taxa & \multicolumn{2}{|c|}{26} & \multicolumn{2}{|c|}{30} & \multicolumn{2}{|c|}{26} \\
\hline Organism & Count & Percent & Count & Percent & Count & Percent \\
\hline \multicolumn{7}{|l|}{ Trichoptera (CADDISFLIES) } \\
\hline \multicolumn{7}{|l|}{ Glossosomatidae } \\
\hline Glossosoma & - & & 2 & $0.14 \%$ & 3 & $0.23 \%$ \\
\hline \multicolumn{7}{|l|}{ Hydropsychidae } \\
\hline Ceratopsyche & 3 & $0.65 \%$ & 77 & $5.56 \%$ & 178 & $13.79 \%$ \\
\hline Cheumatopsyche & 27 & $5.82 \%$ & 169 & $12.19 \%$ & 53 & $4.11 \%$ \\
\hline Hydropsyche & 32 & $6.90 \%$ & 369 & $26.62 \%$ & 280 & $21.69 \%$ \\
\hline Parapsyche & 1 & $.22 \%$ & - & & - & \\
\hline \multicolumn{7}{|l|}{ Hydroptilidae } \\
\hline Hydroptila & 35 & $7.54 \%$ & 2 & $.14 \%$ & 6 & $.46 \%$ \\
\hline Leucotrichia & 2 & $.43 \%$ & 9 & $.65 \%$ & 8 & $.62 \%$ \\
\hline \multicolumn{7}{|l|}{ Leptoceridae } \\
\hline Oecetis & 2 & $.43 \%$ & - & & 1 & $.08 \%$ \\
\hline Mystacides & - & & 2 & $.14 \%$ & - & \\
\hline \multicolumn{7}{|l|}{ Philopotamidae } \\
\hline Chimarra & 2 & $.43 \%$ & 5 & $.36 \%$ & 115 & $8.91 \%$ \\
\hline \multicolumn{7}{|l|}{ Psychomyiidae } \\
\hline Psychomyia & - & & 1 & $.07 \%$ & 1 & $.08 \%$ \\
\hline \multicolumn{7}{|l|}{ Coleoptera (BEETLES) } \\
\hline \multicolumn{7}{|l|}{ Elmidae (RIFFLE BEETLES) } \\
\hline Ancyronyx & 2 & $.43 \%$ & 3 & $.22 \%$ & - & \\
\hline Dubiraphia & - & & 1 & $.07 \%$ & - & \\
\hline Optioservus & 34 & $7.33 \%$ & 30 & $2.16 \%$ & 16 & $1.24 \%$ \\
\hline Oulimnius & 4 & $.86 \%$ & 11 & $.79 \%$ & - & \\
\hline Stenelmis & 75 & $16.16 \%$ & 164 & $11.83 \%$ & 36 & $2.79 \%$ \\
\hline \multicolumn{7}{|l|}{ Hydrophilidae } \\
\hline Berosus & 1 & $.22 \%$ & - & & - & \\
\hline \multicolumn{7}{|c|}{ Psephenidae (WATER PENNIES) } \\
\hline Psephenus & 17 & $3.66 \%$ & 3 & $.22 \%$ & 18 & $1.39 \%$ \\
\hline Diptera (TRUE FLIES) & - & & - & & 1 & $.08 \%$ \\
\hline Chironomidae (MIDGES) & 130 & $28.02 \%$ & 121 & $8.73 \%$ & 230 & $17.82 \%$ \\
\hline \multicolumn{7}{|l|}{ Empididae (DANCE FLIES) } \\
\hline Hemerodromia & 1 & $.22 \%$ & 12 & $.87 \%$ & 2 & $.15 \%$ \\
\hline \multicolumn{7}{|l|}{ Simuliidae (BLACK FLIES) } \\
\hline Prosimulium & - & & - & & 10 & $.77 \%$ \\
\hline Simulium & 19 & $4.09 \%$ & 138 & $9.96 \%$ & 172 & $13.32 \%$ \\
\hline \multicolumn{7}{|l|}{ Tipulidae (CRANE FLIES) } \\
\hline Antocha & 14 & $3.02 \%$ & 46 & $3.32 \%$ & 43 & $3.33 \%$ \\
\hline Dicranota & 1 & $.22 \%$ & 1 & $.07 \%$ & - & \\
\hline Limnophila & - & & - & & 1 & $.08 \%$ \\
\hline
\end{tabular}


Table 5. Benthic-macroinvertebrate data-Continued

01476840 (Site 25) - Goose Creek tributary to East Branch Chester Creek near West Chester, Pa.

\begin{tabular}{|c|c|c|c|c|c|c|}
\hline Date & \multicolumn{2}{|c|}{$10 / 25 / 95$} & \multicolumn{2}{|c|}{$10 / 04 / 96$} & \multicolumn{2}{|c|}{$10 / 03 / 97$} \\
\hline Total count & \multicolumn{2}{|c|}{1,868} & \multicolumn{2}{|c|}{1,298} & \multicolumn{2}{|c|}{1,320} \\
\hline Total number of taxa & \multicolumn{2}{|c|}{18} & \multicolumn{2}{|c|}{19} & \multicolumn{2}{|c|}{12} \\
\hline Organism & Count & Percent & Count & Percent & Count & Percent \\
\hline \multicolumn{7}{|l|}{ Platyhelminthes } \\
\hline \multicolumn{7}{|l|}{ Turbellaria (FLATWORMS) } \\
\hline \multicolumn{7}{|l|}{ Tricladida } \\
\hline Planariidae & 48 & $2.57 \%$ & 25 & $1.93 \%$ & 58 & $4.39 \%$ \\
\hline Nematoda (NEMATODES) & 3 & $.16 \%$ & - & & - & \\
\hline \multicolumn{7}{|l|}{ Nemertea (PROBOSCIS WORMS) } \\
\hline \multicolumn{7}{|l|}{ Enopla } \\
\hline \multicolumn{7}{|l|}{ Hoplonemertea } \\
\hline \multicolumn{7}{|l|}{ Tetrastemmatidae } \\
\hline Prostoma & 1 & $.05 \%$ & 3 & $.23 \%$ & - & \\
\hline \multicolumn{7}{|l|}{ Mollusca } \\
\hline \multicolumn{7}{|l|}{ Gastropoda (SNAILS) } \\
\hline \multicolumn{7}{|l|}{ Basommatophora } \\
\hline \multicolumn{7}{|l|}{ Ancylidae } \\
\hline Ferrissia & 5 & $.27 \%$ & 1 & $.08 \%$ & 1 & $.08 \%$ \\
\hline \multicolumn{7}{|l|}{ Annelida (SEGMENTED WORMS) } \\
\hline Oligochaeta & 6 & $.32 \%$ & - & & - & \\
\hline Tubificida & & & & & & \\
\hline Naididae & 52 & $2.78 \%$ & 15 & $1.16 \%$ & - & \\
\hline Arthropoda & & & & & & \\
\hline Acariformes & & & & & & \\
\hline Hydrachnidia (WATER MITES) & 13 & $.70 \%$ & 23 & $1.77 \%$ & 4 & $.30 \%$ \\
\hline Crustacea & & & & & & \\
\hline Cyclopoida & - & & 1 & $.08 \%$ & - & \\
\hline Isopoda (SOW BUGS) & & & & & & \\
\hline Asellidae & & & & & & \\
\hline Caecidotea & 3 & $.16 \%$ & 1 & $.08 \%$ & - & \\
\hline Insecta & & & & & & \\
\hline Ephemeroptera (MAYFLIES) & & & & & & \\
\hline Baetidae & & & & & & \\
\hline Baetis & - & & 6 & $.46 \%$ & 17 & $1.29 \%$ \\
\hline Ephemerellidae & & & & & & \\
\hline Ephemerella & - & & 1 & $.08 \%$ & - & \\
\hline Heptageniidae & & & & & & \\
\hline Stenonema & 1 & $.05 \%$ & - & & 1 & $.08 \%$ \\
\hline Trichoptera (CADDISFLIES) & & & & & & \\
\hline Hydropsychidae & & & & & & \\
\hline Ceratopsyche & 41 & $2.19 \%$ & 30 & $2.31 \%$ & 201 & $15.23 \%$ \\
\hline Cheumatopsyche & 132 & $7.07 \%$ & 400 & $3.82 \%$ & 311 & $23.56 \%$ \\
\hline Hydropsyche & 210 & $11.24 \%$ & 315 & $24.27 \%$ & 380 & $28.79 \%$ \\
\hline Hydroptilidae & & & & & & \\
\hline Hydroptila & 4 & $.21 \%$ & - & & - & \\
\hline Coleoptera (BEETLES) & & & & & & \\
\hline Elmidae (RIFFLE BEETLES & & & & & & \\
\hline Optioservus & & - & 1 & $.08 \%$ & - & \\
\hline Stenelmis & 11 & $.59 \%$ & 3 & $.23 \%$ & 1 & $.08 \%$ \\
\hline
\end{tabular}


Table 5. Benthic-macroinvertebrate data-Continued

01476840 (Site 25) - Goose Creek tributary to East Branch Chester Creek near West Chester, Pa.-Continued

\begin{tabular}{|c|c|c|c|c|c|c|}
\hline Date & \multicolumn{2}{|c|}{$10 / 25 / 95$} & \multicolumn{2}{|c|}{$10 / 04 / 96$} & \multicolumn{2}{|c|}{$10 / 03 / 97$} \\
\hline Total count & \multicolumn{2}{|c|}{1,868} & \multicolumn{2}{|c|}{1,298} & \multicolumn{2}{|c|}{1,320} \\
\hline Total number of taxa & \multicolumn{2}{|c|}{18} & \multicolumn{2}{|c|}{19} & \multicolumn{2}{|c|}{12} \\
\hline Organism & Count & Percent & Count & Percent & Count & Percent \\
\hline \multicolumn{7}{|l|}{ Diptera (TRUE FLIES) } \\
\hline \multicolumn{7}{|l|}{ Empididae (DANCE FLIES) } \\
\hline $\begin{array}{c}\text { Hemerodromia } \\
\text { Simuliidae (BLACK FLIES) }\end{array}$ & \multicolumn{6}{|c|}{ Simuliidae (BLACK FLIES) } \\
\hline $\begin{array}{c}\text { Simulium } \\
\text { Tipulidae (CRANE FLIES) }\end{array}$ & 250 & $13.38 \%$ & 71 & $5.47 \%$ & 13 & $.98 \%$ \\
\hline Antocha & 14 & $.75 \%$ & 64 & $4.93 \%$ & 23 & $1.74 \%$ \\
\hline Tipula & - & & 4 & $.31 \%$ & - & \\
\hline
\end{tabular}


Table 5. Benthic-macroinvertebrate data-Continued

01476848 (Site 51) - East Branch Chester Creek below Goose Creek near West Chester, Pa.

\begin{tabular}{|c|c|c|c|c|}
\hline Date & \multicolumn{2}{|c|}{$10 / 24 / 95$} & \multicolumn{2}{|c|}{$10 / 06 / 97$} \\
\hline Total count & \multicolumn{2}{|c|}{589} & \multicolumn{2}{|c|}{2,219} \\
\hline Total number of taxa & \multicolumn{2}{|c|}{22} & \multicolumn{2}{|c|}{18} \\
\hline Organism & Count & Percent & Count & Percent \\
\hline \multicolumn{5}{|l|}{ Platyhelminthes } \\
\hline \multicolumn{5}{|l|}{ Turbellaria (FLATWORMS) } \\
\hline \multicolumn{5}{|l|}{ Tricladida } \\
\hline Planariidae & 24 & $4.07 \%$ & 80 & $3.61 \%$ \\
\hline \multicolumn{5}{|l|}{ Nemertea (PROBOSCIS WORMS) } \\
\hline \multicolumn{5}{|l|}{ Enopla } \\
\hline \multicolumn{5}{|l|}{ Hoplonemertea } \\
\hline \multicolumn{5}{|l|}{ Tetrastemmatidae } \\
\hline Prostoma & 1 & $.17 \%$ & - & \\
\hline \multicolumn{5}{|l|}{ Mollusca } \\
\hline \multicolumn{5}{|l|}{ Gastropoda (SNAILS) } \\
\hline \multicolumn{5}{|l|}{ Basommatophora } \\
\hline \multicolumn{5}{|l|}{ Ancylidae } \\
\hline Ferrissia & 2 & $.34 \%$ & - & \\
\hline \multicolumn{5}{|l|}{ Planorbidae } \\
\hline Gyraulus & - & & 1 & $.05 \%$ \\
\hline \multicolumn{5}{|l|}{ Annelida (SEGMENTED WORMS) } \\
\hline \multicolumn{5}{|l|}{ Hirudinea (LEECHES) } \\
\hline \multicolumn{5}{|l|}{ Rhynchobdellida } \\
\hline \multicolumn{5}{|l|}{ Glossiphoniidae } \\
\hline Helobdella & 1 & $.17 \%$ & 一 & \\
\hline \multicolumn{5}{|l|}{ Arthropoda } \\
\hline Acariformes & & & & \\
\hline Hydrachnidia (WATER MITES) & - & & 7 & $.32 \%$ \\
\hline Insecta & & & & \\
\hline Ephemeroptera (MAYFLIES) & & & & \\
\hline Baetidae & & & & \\
\hline Baetis & - & & 28 & $1.26 \%$ \\
\hline Pseudocloeon & - & & 1 & $.05 \%$ \\
\hline Heptageniidae & & & & \\
\hline Stenonema & 1 & $.17 \%$ & - & \\
\hline Leptohyphidae & & & & \\
\hline Tricorythodes & 1 & $.17 \%$ & - & \\
\hline Trichoptera (CADDISFLIES) & & & & \\
\hline Helicopsychidae & & & & \\
\hline Helicopsyche & 3 & $.51 \%$ & 1 & $.05 \%$ \\
\hline Hydropsychidae & & & & \\
\hline Ceratopsyche & 80 & $13.58 \%$ & 670 & $30.19 \%$ \\
\hline Cheumatopsyche & 18 & $3.06 \%$ & 172 & $7.75 \%$ \\
\hline Hydropsyche & 71 & $12.05 \%$ & 634 & $28.57 \%$ \\
\hline Hydroptilidae & & & & \\
\hline Hydroptila & 19 & $3.23 \%$ & 5 & $.23 \%$ \\
\hline Leucotrichia & 6 & $1.02 \%$ & - & \\
\hline
\end{tabular}


Table 5. Benthic-macroinvertebrate data-Continued

01476848 (Site 51) - East Branch Chester Creek below Goose Creek near West Chester, $\mathrm{Pa}$.-Continued

\begin{tabular}{|c|c|c|c|c|}
\hline Date & \multicolumn{2}{|c|}{$10 / 24 / 95$} & \multicolumn{2}{|c|}{$10 / 06 / 97$} \\
\hline Total count & \multicolumn{2}{|c|}{589} & \multicolumn{2}{|c|}{2,219} \\
\hline Total number of taxa & \multicolumn{2}{|c|}{22} & \multicolumn{2}{|c|}{18} \\
\hline Organism & Count & Percent & Count & Percent \\
\hline \multicolumn{5}{|l|}{ Coleoptera (BEETLES) } \\
\hline \multicolumn{5}{|l|}{ Elmidae (RIFFLE BEETLES) } \\
\hline Ancyronyx & - & & 5 & $0.23 \%$ \\
\hline Dubiraphia & 2 & $0.34 \%$ & - & \\
\hline Optioservus & 9 & $1.53 \%$ & 5 & $.23 \%$ \\
\hline Oulimnius & 1 & $.17 \%$ & 4 & $.18 \%$ \\
\hline Stenelmis & 156 & $26.49 \%$ & 164 & $7.39 \%$ \\
\hline \multicolumn{5}{|l|}{ Hydrophilidae } \\
\hline Berosus & 1 & $.17 \%$ & - & \\
\hline \multicolumn{5}{|c|}{ Psephenidae (WATER PENNIES) } \\
\hline Psephenus & 3 & $.51 \%$ & - & \\
\hline \multicolumn{5}{|l|}{ Diptera (TRUE FLIES) } \\
\hline Chironomidae (MIDGES) & 75 & $12.73 \%$ & 351 & $15.82 \%$ \\
\hline \multicolumn{5}{|l|}{ Empididae (DANCE FLIES) } \\
\hline Hemerodromia & - & & 4 & $.18 \%$ \\
\hline \multicolumn{5}{|l|}{ Simuliidae (BLACK FLIES) } \\
\hline Simulium & 16 & $2.72 \%$ & 52 & $2.34 \%$ \\
\hline \multicolumn{5}{|l|}{ Tipulidae (CRANE FLIES) } \\
\hline Antocha & 97 & $16.47 \%$ & 35 & $1.58 \%$ \\
\hline Tipula & 2 & $.34 \%$ & - & \\
\hline
\end{tabular}


Table 5. Benthic-macroinvertebrate data-Continued

01478120 (Site 28) - East Branch White Clay Creek at Avondale, Pa.

\begin{tabular}{|c|c|c|c|c|}
\hline Date & \multicolumn{2}{|c|}{$11 / 09 / 95$} & \multicolumn{2}{|c|}{$11 / 12 / 97$} \\
\hline Total count & \multicolumn{2}{|c|}{1,543} & \multicolumn{2}{|c|}{1,717} \\
\hline Total number of taxa & \multicolumn{2}{|c|}{28} & \multicolumn{2}{|c|}{24} \\
\hline Organism & Count & Percent & Count & Percent \\
\hline \multicolumn{5}{|l|}{ Platyhelminthes } \\
\hline \multicolumn{5}{|l|}{ Turbellaria (FLATWORMS) } \\
\hline \multicolumn{5}{|l|}{ Tricladida } \\
\hline Planariidae & 16 & $1.04 \%$ & 62 & $3.61 \%$ \\
\hline \multicolumn{5}{|l|}{ Nemertea (PROBOSCIS WORMS) } \\
\hline \multicolumn{5}{|l|}{ Enopla } \\
\hline \multicolumn{5}{|l|}{ Hoplonemertea } \\
\hline \multicolumn{5}{|l|}{ Tetrastemmatidae } \\
\hline Prostoma & 2 & $.13 \%$ & - & \\
\hline \multicolumn{5}{|l|}{ Mollusca } \\
\hline \multicolumn{5}{|l|}{ Gastropoda (SNAILS) } \\
\hline \multicolumn{5}{|l|}{ Basommatophora } \\
\hline \multicolumn{5}{|l|}{ Ancylidae } \\
\hline Ferrissia & 1 & $.06 \%$ & - & \\
\hline \multicolumn{5}{|l|}{ Planorbidae } \\
\hline Gyraulus & - & & 1 & $.06 \%$ \\
\hline \multicolumn{5}{|l|}{ Annelida (SEGMENTED WORMS) } \\
\hline Oligochaeta & & & & \\
\hline Lumbriculida & & & & \\
\hline Lumbriculidae & 1 & $.06 \%$ & - & \\
\hline Tubificida & & & & \\
\hline Naididae & 120 & $7.78 \%$ & 2 & $.12 \%$ \\
\hline Arthropoda & & & & \\
\hline Acariformes & & & & \\
\hline Hydrachnidia (WATER MITES) & 18 & $1.17 \%$ & 21 & $1.22 \%$ \\
\hline Crustacea & & & & \\
\hline Cyclopoida & 1 & $.06 \%$ & - & \\
\hline Amphipoda (SCUDS) & & & & \\
\hline Crangonyctidae & & & & \\
\hline Crangonyx & - & & 1 & $.06 \%$ \\
\hline Insecta & & & & \\
\hline Ephemeroptera (MAYFLIES) & & & & \\
\hline Baetidae & & & & \\
\hline Baetis & - & & 1 & $.06 \%$ \\
\hline Ephemerellidae & & & & \\
\hline Ephemerella & 15 & $.97 \%$ & 23 & $1.34 \%$ \\
\hline Heptageniidae & & & & \\
\hline Stenonema & 39 & $2.53 \%$ & 26 & $1.51 \%$ \\
\hline Isonychiidae & & & & \\
\hline Isonychia & 1 & $.06 \%$ & 1 & $.06 \%$ \\
\hline Leptohyphidae & & & & \\
\hline Tricorythodes & 3 & $.19 \%$ & 1 & $.06 \%$ \\
\hline Plecoptera (STONEFLIES) & & & & \\
\hline Capniidae & & & & \\
\hline Allocapnia & 1 & $.06 \%$ & - & \\
\hline Hemiptera (TRUE BUGS) & & & & \\
\hline Veliidae & & & & \\
\hline Rhagovelia & - & & 1 & $.06 \%$ \\
\hline Megaloptera & & & & \\
\hline Sialidae (ALDERFLIES) & & & & \\
\hline Sialis & 1 & $.06 \%$ & - & \\
\hline
\end{tabular}


Table 5. Benthic-macroinvertebrate data-Continued

01478120 (Site 28) - East Branch White Clay Creek at Avondale, Pa.-Continued

\begin{tabular}{|c|c|c|c|c|}
\hline Date & \multicolumn{2}{|c|}{$11 / 09 / 95$} & \multicolumn{2}{|c|}{$11 / 12 / 97$} \\
\hline Total count & \multicolumn{2}{|c|}{1,543} & \multicolumn{2}{|c|}{1,717} \\
\hline Total number of taxa & \multicolumn{2}{|c|}{28} & \multicolumn{2}{|c|}{24} \\
\hline Organism & Count & Percent & Count & Percent \\
\hline \multicolumn{5}{|l|}{ Trichoptera (CADDISFLIES) } \\
\hline \multicolumn{5}{|l|}{ Hydropsychidae } \\
\hline Ceratopsyche & 99 & $6.42 \%$ & 229 & $13.34 \%$ \\
\hline Cheumatopsyche & 63 & $4.08 \%$ & 45 & $2.62 \%$ \\
\hline Hydropsyche & 53 & $3.43 \%$ & 202 & $11.76 \%$ \\
\hline \multicolumn{5}{|l|}{ Hydroptilidae } \\
\hline Hydroptila & 45 & $2.92 \%$ & 87 & $5.07 \%$ \\
\hline Leucotrichia & 2 & $.13 \%$ & - & \\
\hline \multicolumn{5}{|l|}{ Philopotamidae } \\
\hline Chimarra & - & & 1 & $.06 \%$ \\
\hline \multicolumn{5}{|l|}{ Psychomyiidae } \\
\hline Psychomyia & 2 & $.13 \%$ & - & \\
\hline \multicolumn{5}{|l|}{ Coleoptera (BEETLES) } \\
\hline \multicolumn{5}{|l|}{ Elmidae (RIFFLE BEETLES) } \\
\hline Microcylloepus & 1 & $.06 \%$ & - & \\
\hline Optioservus & 31 & $2.01 \%$ & 46 & $2.68 \%$ \\
\hline Oulimnius & - & & 1 & $.06 \%$ \\
\hline Stenelmis & 14 & $.91 \%$ & 15 & $.87 \%$ \\
\hline \multicolumn{5}{|c|}{ Psephenidae (WATER PENNIES) } \\
\hline Psephenus & 4 & $.26 \%$ & 6 & $.35 \%$ \\
\hline \multicolumn{5}{|l|}{ Diptera (TRUE FLIES) } \\
\hline Chironomidae (MIDGES) & 633 & $41.02 \%$ & 460 & $26.79 \%$ \\
\hline \multicolumn{5}{|l|}{ Empididae (DANCE FLIES) } \\
\hline Hemerodromia & 6 & $.39 \%$ & 8 & $.47 \%$ \\
\hline \multicolumn{5}{|l|}{ Simuliidae (BLACK FLIES) } \\
\hline Simulium & 2 & $.13 \%$ & 6 & $.35 \%$ \\
\hline \multicolumn{5}{|l|}{ Tipulidae (CRANE FLIES) } \\
\hline Antocha & 368 & $23.85 \%$ & 471 & $27.43 \%$ \\
\hline Dicranota & 1 & $.06 \%$ & - & \\
\hline
\end{tabular}


Table 5. Benthic-macroinvertebrate data-Continued

01478190 (Site 29) - Middle Branch White Clay Creek at Wickerton, Pa.

\begin{tabular}{|c|c|c|c|c|}
\hline Date & \multicolumn{2}{|c|}{$11 / 09 / 95$} & \multicolumn{2}{|c|}{ 11/12/97 } \\
\hline Total count & \multicolumn{2}{|c|}{1,841} & \multicolumn{2}{|c|}{1,371} \\
\hline Total number of taxa & \multicolumn{2}{|c|}{28} & \multicolumn{2}{|c|}{29} \\
\hline Organism & Count & Percent & Count & Percent \\
\hline \multicolumn{5}{|l|}{ Platyhelminthes } \\
\hline \multicolumn{5}{|l|}{ Turbellaria (FLATWORMS) } \\
\hline \multicolumn{5}{|l|}{ Tricladida } \\
\hline Planariidae & 154 & $8.37 \%$ & 70 & $5.11 \%$ \\
\hline \multicolumn{5}{|l|}{ Annelida (SEGMENTED WORMS) } \\
\hline Oligochaeta & 3 & $.16 \%$ & - & \\
\hline \multicolumn{5}{|l|}{ Tubificida } \\
\hline Naididae & 11 & $.60 \%$ & 1 & $.07 \%$ \\
\hline \multicolumn{5}{|l|}{ Arthropoda } \\
\hline \multicolumn{5}{|l|}{ Acariformes } \\
\hline Hydrachnidia (WATER MITES) & 29 & $1.58 \%$ & 46 & $3.36 \%$ \\
\hline \multicolumn{5}{|l|}{ Crustacea } \\
\hline \multicolumn{5}{|l|}{ Amphipoda (SCUDS) } \\
\hline \multicolumn{5}{|l|}{ Gammaridae } \\
\hline Gammarus & 1 & $.05 \%$ & - & \\
\hline \multicolumn{5}{|l|}{ Isopoda (SOW BUGS) } \\
\hline \multicolumn{5}{|l|}{ Asellidae } \\
\hline Caecidotea & - & & 2 & $.15 \%$ \\
\hline \multicolumn{5}{|l|}{ Insecta } \\
\hline \multicolumn{5}{|l|}{ Ephemeroptera (MAYFLIES) } \\
\hline Baetidae & & & & \\
\hline Baetis & 1 & $.05 \%$ & 17 & $1.24 \%$ \\
\hline Pseudocloeon & - & & 6 & $.44 \%$ \\
\hline Ephemerellidae & & & & \\
\hline Ephemerella & 28 & $1.52 \%$ & 10 & $.73 \%$ \\
\hline Heptageniidae & & & & \\
\hline Stenonema & 5 & $.27 \%$ & 6 & $.44 \%$ \\
\hline Isonychiidae & & & & \\
\hline Isonychia & 2 & $.11 \%$ & 1 & $.07 \%$ \\
\hline Leptophlebiidae & & & & \\
\hline Paraleptophlebia & 3 & $.16 \%$ & - & \\
\hline Plecoptera (STONEFLIES) & & & & \\
\hline Capniidae & & & & \\
\hline Allocapnia & 2 & $.11 \%$ & 1 & $.07 \%$ \\
\hline Perlidae & & & & \\
\hline Acroneuria & - & & 1 & $.07 \%$ \\
\hline Taeniopterygidae & & & & \\
\hline Taeniopteryx & 1 & $.05 \%$ & 1 & $.07 \%$ \\
\hline Megaloptera & & & & \\
\hline Corydalidae (FISHFLIES AI & & & & \\
\hline Nigronia & - & & 2 & $.15 \%$ \\
\hline Trichoptera (CADDISFLIES) & & & & \\
\hline Hydropsychidae & & & & \\
\hline Ceratopsyche & 106 & $5.76 \%$ & 402 & $29.32 \%$ \\
\hline Cheumatopsyche & 65 & $3.53 \%$ & 21 & $1.53 \%$ \\
\hline Hydropsyche & 89 & $4.83 \%$ & 72 & $5.25 \%$ \\
\hline Hydroptilidae & & & & \\
\hline Hydroptila & 25 & $1.36 \%$ & 75 & $5.47 \%$ \\
\hline Leucotrichia & 187 & $10.16 \%$ & 125 & $9.12 \%$ \\
\hline
\end{tabular}


Table 5. Benthic-macroinvertebrate data-Continued

01478190 (Site 29) - Middle Branch White Clay Creek at Wickerton, Pa.-Continued

\begin{tabular}{|c|c|c|c|c|}
\hline Date & \multicolumn{2}{|c|}{$11 / 09 / 95$} & \multicolumn{2}{|c|}{$11 / 12 / 97$} \\
\hline Total count & \multicolumn{2}{|c|}{1,841} & \multicolumn{2}{|c|}{1,371} \\
\hline Total number of taxa & \multicolumn{2}{|c|}{28} & \multicolumn{2}{|c|}{29} \\
\hline Organism & Count & Percent & Count & Percent \\
\hline \multicolumn{5}{|l|}{ Trichoptera (CADDISFLIES) } \\
\hline \multicolumn{5}{|l|}{ Limnephilidae } \\
\hline Hydatophlax & - & & 2 & $0.15 \%$ \\
\hline \multicolumn{5}{|l|}{ Philopotamidae } \\
\hline Chimarra & 188 & $10.21 \%$ & 4 & $.29 \%$ \\
\hline \multicolumn{5}{|l|}{ Polycentropodidae } \\
\hline Cyrnellus & - & & 1 & $.07 \%$ \\
\hline Polycentropus & - & & 1 & $.07 \%$ \\
\hline \multicolumn{5}{|l|}{ Psychomyiidae } \\
\hline Psychomyia & 4 & $.22 \%$ & - & \\
\hline \multicolumn{5}{|l|}{ Coleoptera (BEETLES) } \\
\hline \multicolumn{5}{|l|}{ Elmidae (RIFFLE BEETLES) } \\
\hline Ancyronyx & 1 & $.05 \%$ & - & \\
\hline Optioservus & 59 & $3.20 \%$ & 15 & $1.09 \%$ \\
\hline Stenelmis & 18 & $.98 \%$ & 5 & $.36 \%$ \\
\hline \multicolumn{5}{|c|}{ Psephenidae (WATER PENNIES) } \\
\hline Psephenus & 4 & $.22 \%$ & 2 & $.15 \%$ \\
\hline \multicolumn{5}{|l|}{ Diptera (TRUE FLIES) } \\
\hline Chironomidae (MIDGES) & 620 & $33.68 \%$ & 300 & $21.88 \%$ \\
\hline \multicolumn{5}{|l|}{ Empididae (DANCE FLIES) } \\
\hline Hemerodromia & 13 & $.71 \%$ & 9 & $.66 \%$ \\
\hline \multicolumn{5}{|l|}{ Simuliidae (BLACK FLIES) } \\
\hline Simulium & 1 & $.05 \%$ & 15 & $1.09 \%$ \\
\hline \multicolumn{5}{|l|}{ Tipulidae (CRANE FLIES) } \\
\hline Antocha & 220 & $11.95 \%$ & 158 & $11.52 \%$ \\
\hline Tipula & 1 & $.05 \%$ & - & \\
\hline
\end{tabular}


Table 5. Benthic-macroinvertebrate data-Continued

01478220 (Site 30) - West Branch White Clay Creek near Chesterville, Pa.

\begin{tabular}{|c|c|c|c|c|}
\hline Date & \multicolumn{2}{|c|}{$11 / 09 / 95$} & \multicolumn{2}{|c|}{$11 / 12 / 97$} \\
\hline Total count & \multicolumn{2}{|c|}{637} & \multicolumn{2}{|c|}{1,470} \\
\hline Total number of taxa & \multicolumn{2}{|c|}{30} & \multicolumn{2}{|c|}{27} \\
\hline Organism & Count & Percent & Count & Percent \\
\hline \multicolumn{5}{|l|}{ Platyhelminthes } \\
\hline \multicolumn{5}{|l|}{ Turbellaria (FLATWORMS) } \\
\hline \multicolumn{5}{|l|}{ Tricladida } \\
\hline Planariidae & 17 & $2.67 \%$ & 4 & $0.27 \%$ \\
\hline Nematoda (NEMATODES) & - & & 1 & $.07 \%$ \\
\hline \multicolumn{5}{|l|}{ Nemertea (PROBOSCIS WORMS) } \\
\hline \multicolumn{5}{|l|}{ Enopla } \\
\hline \multicolumn{5}{|l|}{ Hoplonemertea } \\
\hline \multicolumn{5}{|l|}{ Tetrastemmatidae } \\
\hline Prostoma & 1 & $.16 \%$ & - & \\
\hline \multicolumn{5}{|l|}{ Mollusca } \\
\hline \multicolumn{5}{|l|}{ Gastropoda (SNAILS) } \\
\hline \multicolumn{5}{|l|}{ Basommatophora } \\
\hline \multicolumn{5}{|l|}{ Ancylidae } \\
\hline Ferrissia & - & & 1 & $.07 \%$ \\
\hline \multicolumn{5}{|l|}{ Annelida (SEGMENTED WORMS) } \\
\hline \multicolumn{5}{|l|}{ Oligochaeta } \\
\hline \multicolumn{5}{|l|}{ Lumbriculida } \\
\hline Lumbriculidae & 1 & $.16 \%$ & - & \\
\hline Tubificida & & & & \\
\hline Naididae & 8 & $1.26 \%$ & - & \\
\hline Arthropoda & & & & \\
\hline Acariformes & & & & \\
\hline Hydrachnidia (WATER MITES) & 31 & $4.87 \%$ & 79 & $5.37 \%$ \\
\hline Crustacea & & & & \\
\hline Cyclopoida & 1 & $.16 \%$ & - & \\
\hline Insecta & & & & \\
\hline Ephemeroptera (MAYFLIES) & & & & \\
\hline Baetidae & & & & \\
\hline Pseudocloeon & 2 & $.31 \%$ & 2 & $.14 \%$ \\
\hline Ephemerellidae & & & & \\
\hline Ephemerella & 21 & $3.30 \%$ & - & \\
\hline Heptageniidae & & & & \\
\hline Epeorus & 1 & $.16 \%$ & - & \\
\hline Stenonema & 32 & $5.02 \%$ & 28 & $1.90 \%$ \\
\hline Isonychiidae & & & & \\
\hline Isonychia & 12 & $1.88 \%$ & 26 & $1.77 \%$ \\
\hline Plecoptera (STONEFLIES) & & & & \\
\hline Capniidae & & & & \\
\hline Allocapnia & 7 & $1.10 \%$ & - & \\
\hline Leuctridae & & & & \\
\hline Paraleuctra & - & & 2 & $.14 \%$ \\
\hline Perlidae & & & & \\
\hline Acroneuria & 1 & $.16 \%$ & 3 & $.20 \%$ \\
\hline Taeniopterygidae & & & & \\
\hline Strophopteryx & - & & 3 & $.20 \%$ \\
\hline Taeniopteryx & 12 & $1.88 \%$ & - & \\
\hline Megaloptera & & & & \\
\hline Corydalidae (FISHFLIES ANL & & & & \\
\hline Corydalus & - & & 1 & $.07 \%$ \\
\hline
\end{tabular}


Table 5. Benthic-macroinvertebrate data-Continued

01478220 (Site 30) - West Branch White Clay Creek near Chesterville, Pa.-Continued

\begin{tabular}{|c|c|c|c|c|}
\hline Date & \multicolumn{2}{|c|}{$11 / 09 / 95$} & \multicolumn{2}{|c|}{$11 / 12 / 97$} \\
\hline Total count & \multicolumn{2}{|c|}{637} & \multicolumn{2}{|c|}{1,470} \\
\hline Total number of taxa & \multicolumn{2}{|c|}{30} & \multicolumn{2}{|c|}{27} \\
\hline Organism & Count & Percent & Count & Percent \\
\hline \multicolumn{5}{|l|}{ Trichoptera (CADDISFLIES) } \\
\hline \multicolumn{5}{|l|}{ Glossosomatidae } \\
\hline Glossosoma & 2 & $0.31 \%$ & 8 & $0.54 \%$ \\
\hline \multicolumn{5}{|l|}{ Hydropsychidae } \\
\hline Ceratopsyche & 47 & $7.38 \%$ & 426 & $28.98 \%$ \\
\hline Cheumatopsyche & 71 & $11.15 \%$ & 54 & $3.67 \%$ \\
\hline Hydropsyche & 5 & $.78 \%$ & 170 & $11.56 \%$ \\
\hline \multicolumn{5}{|l|}{ Hydroptilidae } \\
\hline Hydroptila & 9 & $1.41 \%$ & 55 & $3.74 \%$ \\
\hline Leucotrichia & 2 & $.31 \%$ & 4 & $.27 \%$ \\
\hline \multicolumn{5}{|l|}{ Philopotamidae } \\
\hline Chimarra & 17 & $2.67 \%$ & 1 & $.07 \%$ \\
\hline Dolophilodes & - & & 1 & $.07 \%$ \\
\hline \multicolumn{5}{|l|}{ Polycentropodidae } \\
\hline Neureclipsis & 1 & $.16 \%$ & 1 & $.07 \%$ \\
\hline \multicolumn{5}{|l|}{ Psychomyiidae } \\
\hline Psychomyia & 3 & $.47 \%$ & - & \\
\hline \multicolumn{5}{|l|}{ Uenoidae } \\
\hline Neophylax & 2 & $.31 \%$ & - & \\
\hline \multicolumn{5}{|l|}{ Coleoptera (BEETLES) } \\
\hline \multicolumn{5}{|l|}{ Elmidae (RIFFLE BEETLES) } \\
\hline Optioservus & 6 & $.94 \%$ & 7 & $.48 \%$ \\
\hline Oulimnius & - & & 1 & $.07 \%$ \\
\hline Stenelmis & 2 & $.31 \%$ & - & \\
\hline \multicolumn{5}{|l|}{ Psephenidae (WATER PENNIES) } \\
\hline Psephenus & - & & 2 & $.14 \%$ \\
\hline \multicolumn{5}{|l|}{ Diptera (TRUE FLIES) } \\
\hline Chironomidae (MIDGES) & 230 & $36.11 \%$ & 303 & $20.61 \%$ \\
\hline \multicolumn{5}{|l|}{ Empididae (DANCE FLIES) } \\
\hline Hemerodromia & 15 & $2.35 \%$ & 27 & $1.84 \%$ \\
\hline \multicolumn{5}{|l|}{ Simuliidae (BLACK FLIES) } \\
\hline Simulium & 2 & $.31 \%$ & 3 & $.20 \%$ \\
\hline \multicolumn{5}{|l|}{ Tipulidae (CRANE FLIES) } \\
\hline Antocha & 76 & $11.93 \%$ & 257 & $17.48 \%$ \\
\hline
\end{tabular}


Table 5. Benthic-macroinvertebrate data-Continued

01479680 (Site 27) - West Branch Red Clay Creek at Kennett Square, Pa.

\begin{tabular}{|c|c|c|c|c|c|c|}
\hline Date & \multicolumn{2}{|c|}{ 10/18/95 } & \multicolumn{2}{|c|}{$11 / 05 / 96$} & \multicolumn{2}{|c|}{$10 / 14 / 97$} \\
\hline Total count & \multicolumn{2}{|c|}{323} & \multicolumn{2}{|c|}{178} & \multicolumn{2}{|c|}{790} \\
\hline Total number of taxa & \multicolumn{2}{|c|}{22} & \multicolumn{2}{|c|}{15} & \multicolumn{2}{|c|}{26} \\
\hline Organism & Count & Percent & Count & Percent & Count & Percent \\
\hline \multicolumn{7}{|l|}{ Platyhelminthes } \\
\hline \multicolumn{7}{|l|}{ Turbellaria (FLATWORMS) } \\
\hline \multicolumn{7}{|l|}{ Tricladida } \\
\hline Planariidae & 9 & $2.79 \%$ & - & & 2 & $0.25 \%$ \\
\hline \multicolumn{7}{|l|}{ Nemertea (PROBOSCIS WORMS) } \\
\hline \multicolumn{7}{|l|}{ Enopla } \\
\hline \multicolumn{7}{|l|}{ Hoplonemertea } \\
\hline \multicolumn{7}{|l|}{ Tetrastemmatidae } \\
\hline Prostoma & 3 & $.93 \%$ & - & & 5 & $.63 \%$ \\
\hline \multicolumn{7}{|l|}{ Mollusca } \\
\hline \multicolumn{7}{|l|}{ Gastropoda (SNAILS) } \\
\hline \multicolumn{7}{|l|}{ Basommatophora } \\
\hline Ancylidae & & & & & & \\
\hline Ferrissia & 6 & $1.86 \%$ & 1 & $0.56 \%$ & 5 & $.63 \%$ \\
\hline Planorbidae & & & & & & \\
\hline Gyraulus & 2 & $.62 \%$ & - & & 3 & $.38 \%$ \\
\hline Annelida (SEGMENTED WORMS) & & & & & & \\
\hline Oligochaeta & - & & - & & 2 & $.25 \%$ \\
\hline Tubificida & & & & & & \\
\hline Naididae & 15 & $4.64 \%$ & 25 & $14.04 \%$ & 150 & $18.99 \%$ \\
\hline Arthropoda & & & & & & \\
\hline Acariformes & & & & & & \\
\hline Hydrachnidia (WATER MITES) & 52 & $16.10 \%$ & 6 & $3.37 \%$ & 105 & $13.29 \%$ \\
\hline Crustacea & & & & & & \\
\hline Cyclopoida & - & & 1 & $.56 \%$ & 1 & $.13 \%$ \\
\hline Amphipoda (SCUDS) & & & & & & \\
\hline Crangonyctidae & & & & & & \\
\hline Crangonyx & - & & - & & 1 & $.13 \%$ \\
\hline Gammaridae & & & & & & \\
\hline Gammarus & 1 & $.31 \%$ & - & & - & \\
\hline Isopoda (SOW BUGS) & & & & & & \\
\hline Asellidae & & & & & & \\
\hline Caecidotea & 2 & $.62 \%$ & - & & - & \\
\hline Lirceus & - & & - & & 2 & $.25 \%$ \\
\hline Insecta & & & & & & \\
\hline Ephemeroptera (MAYFLIES) & & & & & & \\
\hline Baetidae & & & & & & \\
\hline Baetis & - & & - & & 1 & $.13 \%$ \\
\hline Pseudocloeon & - & & - & & 1 & $.13 \%$ \\
\hline Ephemerellidae & & & & & & \\
\hline Ephemerella & 3 & $.93 \%$ & 1 & $.56 \%$ & 1 & $.13 \%$ \\
\hline Heptageniidae & - & & 1 & $.56 \%$ & - & \\
\hline Stenacron & 1 & $.31 \%$ & - & & - & \\
\hline Stenonema & - & & - & & 2 & $.25 \%$ \\
\hline Leptohyphidae & & & & & & \\
\hline Tricorythodes & - & & 1 & $.56 \%$ & - & \\
\hline Plecoptera (STONEFLIES) & & & & & & \\
\hline Taeniopterygidae & & & & & & \\
\hline Taeniopteryx & - & & - & & 1 & $.13 \%$ \\
\hline
\end{tabular}


Table 5. Benthic-macroinvertebrate data-Continued

01479680 (Site 27) - West Branch Red Clay Creek at Kennett Square, Pa.-Continued

\begin{tabular}{|c|c|c|c|c|c|c|}
\hline Date & \multicolumn{2}{|c|}{$10 / 18 / 95$} & \multicolumn{2}{|c|}{$11 / 05 / 96$} & \multicolumn{2}{|c|}{$10 / 14 / 97$} \\
\hline Total count & \multicolumn{2}{|c|}{323} & \multicolumn{2}{|c|}{178} & \multicolumn{2}{|c|}{790} \\
\hline Total number of taxa & \multicolumn{2}{|c|}{22} & \multicolumn{2}{|c|}{15} & \multicolumn{2}{|c|}{26} \\
\hline Organism & Count & Percent & Count & Percent & Count & Percent \\
\hline \multicolumn{7}{|l|}{ Megaloptera } \\
\hline \multicolumn{7}{|c|}{ Corydalidae (FISHFLIES AND DOBSONFLIES) } \\
\hline Nigronia & - & & - & & 3 & $0.38 \%$ \\
\hline \multicolumn{7}{|l|}{ Trichoptera (CADDISFLIES) } \\
\hline \multicolumn{7}{|l|}{ Hydropsychidae } \\
\hline Ceratopsyche & 8 & $2.48 \%$ & 15 & $8.43 \%$ & 43 & $5.44 \%$ \\
\hline Cheumatopsyche & 18 & $5.57 \%$ & 8 & $4.49 \%$ & 48 & $6.08 \%$ \\
\hline Hydropsyche & 15 & $4.64 \%$ & 1 & $.56 \%$ & 19 & $2.41 \%$ \\
\hline \multicolumn{7}{|l|}{ Hydroptilidae } \\
\hline Leucotrichia & - & & - & & 1 & $.13 \%$ \\
\hline \multicolumn{7}{|l|}{ Leptoceridae } \\
\hline Mystacides & 1 & $.31 \%$ & - & & - & \\
\hline \multicolumn{7}{|l|}{ Coleoptera (BEETLES) } \\
\hline \multicolumn{7}{|l|}{ Elmidae (RIFFLE BEETLES) } \\
\hline Ancyronyx & 1 & $.31 \%$ & - & & - & \\
\hline Dubiraphia & - & & 1 & $.56 \%$ & - & \\
\hline Optioservus & 11 & $3.41 \%$ & 1 & $.56 \%$ & - & \\
\hline Oulimnius & 1 & $.31 \%$ & - & & 5 & $.63 \%$ \\
\hline Stenelmis & 9 & $2.79 \%$ & - & & 11 & $1.39 \%$ \\
\hline \multicolumn{7}{|c|}{ Psephenidae (WATER PENNIES) } \\
\hline Psephenus & 2 & $.62 \%$ & - & & - & \\
\hline \multicolumn{7}{|l|}{ Diptera (TRUE FLIES) } \\
\hline Chironomidae (MIDGES) & 103 & $31.89 \%$ & 91 & $51.12 \%$ & 260 & $32.91 \%$ \\
\hline \multicolumn{7}{|l|}{ Empididae (DANCE FLIES) } \\
\hline Hemerodromia & 16 & $4.95 \%$ & 1 & $.56 \%$ & 18 & $2.28 \%$ \\
\hline \multicolumn{7}{|l|}{ Simuliidae (BLACK FLIES) } \\
\hline Simulium & - & & - & & 1 & $.13 \%$ \\
\hline \multicolumn{7}{|l|}{ Tipulidae (CRANE FLIES) } \\
\hline Antocha & 44 & $13.62 \%$ & 24 & $13.48 \%$ & 99 & $12.53 \%$ \\
\hline
\end{tabular}


Table 5. Benthic-macroinvertebrate data-Continued

01479800 (Site 26) - East Branch Red Clay Creek near Five Point, Pa.

\begin{tabular}{|c|c|c|c|c|c|c|}
\hline Date & \multicolumn{2}{|c|}{$10 / 18 / 95$} & \multicolumn{2}{|c|}{$11 / 05 / 96$} & \multicolumn{2}{|c|}{ 10/14/97 } \\
\hline Total count & \multicolumn{2}{|c|}{514} & \multicolumn{2}{|c|}{1,240} & \multicolumn{2}{|c|}{1,213} \\
\hline Total number of taxa & \multicolumn{2}{|c|}{22} & \multicolumn{2}{|c|}{22} & \multicolumn{2}{|c|}{17} \\
\hline Organism & Count & Percent & Count & Percent & Count & Percent \\
\hline \multicolumn{7}{|l|}{ Platyhelminthes } \\
\hline \multicolumn{7}{|l|}{ Turbellaria (FLATWORMS) } \\
\hline \multicolumn{7}{|l|}{ Tricladida } \\
\hline Planariidae & 31 & $6.03 \%$ & 35 & $2.82 \%$ & 28 & $2.31 \%$ \\
\hline \multicolumn{7}{|l|}{ Nemertea (PROBOSCIS WORMS) } \\
\hline \multicolumn{7}{|l|}{ Enopla } \\
\hline \multicolumn{7}{|l|}{ Hoplonemertea } \\
\hline \multicolumn{7}{|l|}{ Tetrastemmatidae } \\
\hline Prostoma & 1 & $.19 \%$ & 2 & $.16 \%$ & - & \\
\hline \multicolumn{7}{|l|}{ Mollusca } \\
\hline \multicolumn{7}{|l|}{ Gastropoda (SNAILS) } \\
\hline \multicolumn{7}{|l|}{ Basommatophora } \\
\hline Ancylidae & & & & & & \\
\hline Ferrissia & 3 & $.58 \%$ & 8 & $.65 \%$ & - & \\
\hline Planorbidae & & & & & & \\
\hline Gyraulus & 1 & $.19 \%$ & - & & - & \\
\hline Annelida (SEGMENTED WORMS) & & & & & & \\
\hline Oligochaeta & & & & & & \\
\hline Tubificida & & & & & & \\
\hline Naididae & 2 & $.39 \%$ & 18 & $1.45 \%$ & - & \\
\hline Arthropoda & & & & & & \\
\hline Acariformes & & & & & & \\
\hline Hydrachnidia (WATER MITES) & 8 & $1.56 \%$ & 22 & $1.77 \%$ & 14 & $1.15 \%$ \\
\hline Insecta & & & & & & \\
\hline Ephemeroptera (MAYFLIES) & & & & & & \\
\hline Baetidae & & & & & & \\
\hline Baetis & - & & - & & 7 & $.58 \%$ \\
\hline Ephemerellidae & & & & & & \\
\hline Ephemerella & 12 & $2.33 \%$ & - & & 2 & $.16 \%$ \\
\hline Heptageniidae & & & & & & \\
\hline Epeorus & - & & - & & 1 & $.08 \%$ \\
\hline Stenonema & 11 & $2.14 \%$ & 1 & $.08 \%$ & - & \\
\hline Plecoptera (STONEFLIES) & & & & & & \\
\hline Capniidae & & & & & & \\
\hline Allocapnia & - & & 1 & $.08 \%$ & - & \\
\hline Taeniopterygidae & & & & & & \\
\hline Taeniopteryx & - & & 1 & $.08 \%$ & - & \\
\hline Megaloptera & & & & & & \\
\hline Corydalidae (FISHFLIES AI & & & & & & \\
\hline Corydalus & - & & 1 & $.08 \%$ & 1 & $.08 \%$ \\
\hline Trichoptera (CADDISFLIES) & & & & & & \\
\hline Hydropsychidae & & & & & & \\
\hline Ceratopsyche & 74 & $14.40 \%$ & 316 & $25.48 \%$ & 466 & $38.42 \%$ \\
\hline Cheumatopsyche & 74 & $14.40 \%$ & 33 & $2.66 \%$ & 2 & $.16 \%$ \\
\hline Hydropsyche & 75 & $14.59 \%$ & 8 & $.65 \%$ & 2 & $.16 \%$ \\
\hline Hydroptilidae & & & & & & \\
\hline Hydroptila & 1 & $.19 \%$ & - & & - & \\
\hline Leucotrichia & 2 & $.39 \%$ & - & & 1 & $.08 \%$ \\
\hline Philopotamidae & & & & & & \\
\hline Chimarra & 1 & $.19 \%$ & 1 & $.08 \%$ & - & \\
\hline
\end{tabular}


Table 5. Benthic-macroinvertebrate data-Continued

01479800 (Site 26) - East Branch Red Clay Creek near Five Point, Pa.-Continued

\begin{tabular}{|c|c|c|c|c|c|c|}
\hline Date & \multicolumn{2}{|c|}{$10 / 18 / 95$} & \multicolumn{2}{|c|}{$11 / 05 / 96$} & \multicolumn{2}{|c|}{$10 / 14 / 97$} \\
\hline Total count & \multicolumn{2}{|c|}{514} & \multicolumn{2}{|c|}{1,240} & \multicolumn{2}{|c|}{1,213} \\
\hline Total number of taxa & \multicolumn{2}{|c|}{22} & \multicolumn{2}{|c|}{22} & \multicolumn{2}{|c|}{17} \\
\hline Organism & Count & Percent & Count & Percent & Count & Percent \\
\hline \multicolumn{7}{|l|}{ Coleoptera (BEETLES) } \\
\hline \multicolumn{7}{|l|}{ Elmidae (RIFFLE BEETLES) } \\
\hline Ancyronyx & - & & 2 & $0.16 \%$ & - & \\
\hline Optioservus & 50 & $9.73 \%$ & 55 & $4.44 \%$ & 120 & $9.89 \%$ \\
\hline Oulimnius & 1 & $.19 \%$ & 2 & $.16 \%$ & 7 & $.58 \%$ \\
\hline Stenelmis & 13 & $2.53 \%$ & 12 & $.97 \%$ & 25 & $2.06 \%$ \\
\hline \multicolumn{7}{|c|}{ Psephenidae (WATER PENNIES) } \\
\hline Psephenus & 3 & $.58 \%$ & - & & - & \\
\hline \multicolumn{7}{|l|}{ Diptera (TRUE FLIES) } \\
\hline Chironomidae (MIDGES) & 84 & $16.34 \%$ & 412 & $33.23 \%$ & 357 & $29.43 \%$ \\
\hline Empididae (DANCE FLIES) & - & & 4 & $.32 \%$ & - & \\
\hline Hemerodromia & 10 & $1.95 \%$ & 20 & $1.61 \%$ & 7 & $.58 \%$ \\
\hline \multicolumn{7}{|l|}{ Simuliidae (BLACK FLIES) } \\
\hline Simulium & 3 & $.58 \%$ & 176 & $14.19 \%$ & 21 & $1.73 \%$ \\
\hline \multicolumn{7}{|l|}{ Tipulidae (CRANE FLIES) } \\
\hline Antocha & 54 & $10.51 \%$ & 110 & $8.87 \%$ & 152 & $12.53 \%$ \\
\hline
\end{tabular}


Table 5. Benthic-macroinvertebrate data-Continued

01480434 (Site 37) - West Branch Brandywine Creek at Rock Run, Pa.

\begin{tabular}{|c|c|c|c|c|}
\hline Date & \multicolumn{2}{|c|}{$10 / 17 / 95$} & \multicolumn{2}{|c|}{$10 / 29 / 96$} \\
\hline Total count & \multicolumn{2}{|c|}{440} & \multicolumn{2}{|c|}{511} \\
\hline Total number of taxa & \multicolumn{2}{|c|}{36} & \multicolumn{2}{|c|}{27} \\
\hline Organism & Count & Percent & Count & Percent \\
\hline \multicolumn{5}{|l|}{ Platyhelminthes } \\
\hline \multicolumn{5}{|l|}{ Turbellaria (FLATWORMS) } \\
\hline \multicolumn{5}{|l|}{ Tricladida } \\
\hline Planariidae & 4 & $0.91 \%$ & 5 & $0.98 \%$ \\
\hline \multicolumn{5}{|l|}{ Nemertea (PROBOSCIS WORMS) } \\
\hline \multicolumn{5}{|l|}{ Enopla } \\
\hline \multicolumn{5}{|l|}{ Hoplonemertea } \\
\hline \multicolumn{5}{|l|}{ Tetrastemmatidae } \\
\hline Prostoma & 2 & $.45 \%$ & - & \\
\hline \multicolumn{5}{|l|}{ Mollusca } \\
\hline \multicolumn{5}{|l|}{ Gastropoda (SNAILS) } \\
\hline \multicolumn{5}{|l|}{ Basommatophora } \\
\hline \multicolumn{5}{|l|}{ Ancylidae } \\
\hline Ferrissia & 12 & $2.73 \%$ & - & \\
\hline \multicolumn{5}{|l|}{ Bivalvia (CLAMS) } \\
\hline \multicolumn{5}{|l|}{ Veneroida } \\
\hline Sphaeriidae & 4 & $.91 \%$ & - & \\
\hline \multicolumn{5}{|l|}{ Annelida (SEGMENTED WORMS) } \\
\hline \multicolumn{5}{|l|}{ Oligochaeta } \\
\hline Tubificida & & & & \\
\hline Naididae & 5 & $1.14 \%$ & 4 & $.78 \%$ \\
\hline Arthropoda & & & & \\
\hline Acariformes & & & & \\
\hline Hydrachnidia (WATER MITES) & 17 & $3.86 \%$ & 16 & $3.13 \%$ \\
\hline Crustacea & & & & \\
\hline Podocopa (SEED SHRIMP) & 2 & $.45 \%$ & - & \\
\hline Insecta & & & & \\
\hline Ephemeroptera (MAYFLIES) & & & & \\
\hline Baetidae & & & & \\
\hline Baetis & 1 & $.23 \%$ & - & \\
\hline Pseudocloeon & - & & 4 & $.78 \%$ \\
\hline Ephemerellidae & & & & \\
\hline Ephemerella & 34 & $7.73 \%$ & 23 & $4.50 \%$ \\
\hline Heptageniidae & & & & \\
\hline Epeorus & - & & 4 & $.78 \%$ \\
\hline Stenonema & 35 & $7.95 \%$ & 11 & $2.15 \%$ \\
\hline Isonychiidae & & & & \\
\hline Isonychia & 7 & $1.59 \%$ & 9 & $1.76 \%$ \\
\hline Plecoptera (STONEFLIES) & & & & \\
\hline Capniidae & & & & \\
\hline Allocapnia & 3 & $.68 \%$ & 130 & $25.44 \%$ \\
\hline Chloroperlidae & 1 & $.23 \%$ & - & \\
\hline Perlidae & & & & \\
\hline Acroneuria & 1 & $.23 \%$ & - & \\
\hline Agnetina & 1 & $.23 \%$ & - & \\
\hline Taeniopterygidae & & & & \\
\hline Taeniopteryx & 4 & $.91 \%$ & 10 & $1.96 \%$ \\
\hline
\end{tabular}


Table 5. Benthic-macroinvertebrate data-Continued

01480434 (Site 37) - West Branch Brandywine Creek at Rock Run, Pa. —Continued

\begin{tabular}{|c|c|c|c|c|}
\hline Date & \multicolumn{2}{|c|}{ 10/17/95 } & \multicolumn{2}{|c|}{ 10/29/96 } \\
\hline Total count & \multicolumn{2}{|c|}{440} & \multicolumn{2}{|c|}{511} \\
\hline Total number of taxa & \multicolumn{2}{|c|}{36} & \multicolumn{2}{|c|}{27} \\
\hline Organism & Count & Percent & Count & Percent \\
\hline \multicolumn{5}{|c|}{ Megaloptera } \\
\hline \multicolumn{5}{|c|}{ Corydalidae (FISHFLIES AND DOBSONFLIES) } \\
\hline Corydalus & 2 & $0.45 \%$ & - & \\
\hline \multicolumn{5}{|l|}{ Trichoptera (CADDISFLIES) } \\
\hline \multicolumn{5}{|l|}{ Apataniidae } \\
\hline Apatania & 3 & $.68 \%$ & 1 & $0.20 \%$ \\
\hline \multicolumn{5}{|l|}{ Brachycentridae } \\
\hline Micrasema & 1 & $.23 \%$ & 4 & $.78 \%$ \\
\hline \multicolumn{5}{|l|}{ Glossosomatidae } \\
\hline Glossosoma & - & & 1 & $.20 \%$ \\
\hline \multicolumn{5}{|l|}{ Hydropsychidae } \\
\hline Ceratopsyche & 20 & $4.55 \%$ & 77 & $15.07 \%$ \\
\hline Cheumatopsyche & 48 & $10.91 \%$ & 62 & $12.13 \%$ \\
\hline Hydropsyche & 3 & $.68 \%$ & 13 & $2.54 \%$ \\
\hline \multicolumn{5}{|l|}{ Hydroptilidae } \\
\hline Hydroptila & 1 & $.23 \%$ & - & \\
\hline Leucotrichia & 11 & $2.50 \%$ & 5 & $.98 \%$ \\
\hline \multicolumn{5}{|l|}{ Limnephilidae } \\
\hline Hydatophylax & - & & 1 & $.20 \%$ \\
\hline \multicolumn{5}{|l|}{ Philopotamidae } \\
\hline Chimarra & 20 & $4.55 \%$ & 10 & $1.96 \%$ \\
\hline \multicolumn{5}{|l|}{ Polycentropodidae } \\
\hline Cyrnellus & 10 & $2.27 \%$ & 12 & $2.35 \%$ \\
\hline \multicolumn{5}{|l|}{ Lepidoptera } \\
\hline \multicolumn{5}{|l|}{ Pyralidae (MOTHS) } \\
\hline Petrophila & 1 & $.23 \%$ & 1 & $.20 \%$ \\
\hline \multicolumn{5}{|l|}{ Coleoptera (BEETLES) } \\
\hline \multicolumn{5}{|l|}{ Elmidae (RIFFLE BEETLES) } \\
\hline Ancyronyx & 1 & $.23 \%$ & - & \\
\hline Optioservus & 23 & $5.23 \%$ & 6 & $1.17 \%$ \\
\hline Promoresia & 2 & $.45 \%$ & - & \\
\hline Stenelmis & 6 & $1.36 \%$ & 1 & $.20 \%$ \\
\hline \multicolumn{5}{|c|}{ Psephenidae (WATER PENNIES) } \\
\hline Psephenus & 13 & $2.95 \%$ & - & \\
\hline \multicolumn{5}{|l|}{ Diptera (TRUE FLIES) } \\
\hline Chironomidae (MIDGES) & 125 & $28.41 \%$ & 73 & $14.29 \%$ \\
\hline \multicolumn{5}{|l|}{ Empididae (DANCE FLIES) } \\
\hline Hemerodromia & 6 & $1.36 \%$ & 3 & $.59 \%$ \\
\hline Simuliidae (BLACK FLIES) & & & & \\
\hline Simulium & 2 & $.45 \%$ & 9 & $1.76 \%$ \\
\hline Tipulidae (CRANE FLIES) & & & & \\
\hline Antocha & 9 & $2.05 \%$ & 16 & $3.13 \%$ \\
\hline
\end{tabular}


Table 5. Benthic-macroinvertebrate data-Continued

01480629 (Site 46) - Buck Run at Doe Run, Pa.

\begin{tabular}{|c|c|c|c|c|}
\hline Date & \multicolumn{2}{|c|}{ 11/28/95 } & \multicolumn{2}{|c|}{$11 / 06 / 97$} \\
\hline Total count & \multicolumn{2}{|c|}{674} & \multicolumn{2}{|c|}{1,405} \\
\hline Total number of taxa & \multicolumn{2}{|c|}{27} & \multicolumn{2}{|c|}{31} \\
\hline Organism & Count & Percent & Count & Percent \\
\hline \multicolumn{5}{|l|}{ Platyhelminthes } \\
\hline \multicolumn{5}{|l|}{ Turbellaria (FLATWORMS) } \\
\hline \multicolumn{5}{|l|}{ Tricladida } \\
\hline Planariidae & 54 & $8.01 \%$ & 83 & $5.91 \%$ \\
\hline Nematoda (NEMATODES) & 1 & $.15 \%$ & - & \\
\hline \multicolumn{5}{|l|}{ Nemertea (PROBOSCIS WORMS) } \\
\hline \multicolumn{5}{|l|}{ Enopla } \\
\hline \multicolumn{5}{|l|}{ Hoplonemertea } \\
\hline \multicolumn{5}{|l|}{ Tetrastemmatidae } \\
\hline Prostoma & 1 & $.15 \%$ & 2 & $.14 \%$ \\
\hline \multicolumn{5}{|l|}{ Mollusca } \\
\hline \multicolumn{5}{|l|}{ Gastropoda (SNAILS) } \\
\hline \multicolumn{5}{|l|}{ Basommatophora } \\
\hline \multicolumn{5}{|l|}{ Ancylidae } \\
\hline Ferrissia & 2 & $.30 \%$ & 4 & $.28 \%$ \\
\hline \multicolumn{5}{|l|}{ Physidae } \\
\hline Physa & 1 & $.15 \%$ & - & \\
\hline \multicolumn{5}{|l|}{ Planorbidae } \\
\hline Gyraulus & - & & 2 & $.14 \%$ \\
\hline \multicolumn{5}{|l|}{ Bivalvia (CLAMS) } \\
\hline Veneroida & & & & \\
\hline Sphaeriidae & 2 & $.30 \%$ & 1 & $.07 \%$ \\
\hline Annelida (SEGMENTED WORMS) & & & & \\
\hline Oligochaeta & & & & \\
\hline Tubificida & & & & \\
\hline Naididae & 1 & $.15 \%$ & 5 & $.36 \%$ \\
\hline Arthropoda & & & & \\
\hline Acariformes & & & & \\
\hline Hydrachnidia (WATER MITES) & 6 & $.89 \%$ & 38 & $2.70 \%$ \\
\hline Crustacea & & & & \\
\hline Isopoda (SOW BUGS) & & & & \\
\hline Asellidae & & & & \\
\hline Lirceus & - & & 2 & $.14 \%$ \\
\hline Insecta & & & & \\
\hline Ephemeroptera (MAYFLIES) & & & & \\
\hline Baetidae & & & & \\
\hline Pseudocloeon & - & & 1 & $.07 \%$ \\
\hline Caenidae & & & & \\
\hline Caenis & 1 & $.15 \%$ & - & \\
\hline Ephemerellidae & & & & \\
\hline Ephemerella & 62 & $9.20 \%$ & 100 & $7.12 \%$ \\
\hline Heptageniidae & & & & \\
\hline Epeorus & - & & 5 & $.36 \%$ \\
\hline Stenonema & 12 & $1.78 \%$ & 35 & $2.49 \%$ \\
\hline Isonychiidae & & & & \\
\hline Isonychia & 26 & $3.86 \%$ & 9 & $.64 \%$ \\
\hline Megaloptera & & & & \\
\hline Corydalidae (FISHFLIES AN & & & & \\
\hline Corydalus & 1 & $.15 \%$ & - & \\
\hline
\end{tabular}


Table 5. Benthic-macroinvertebrate data-Continued

01480629 (Site 46) - Buck Run at Doe Run, Pa.-Continued

\begin{tabular}{|c|c|c|c|c|}
\hline Date & \multicolumn{2}{|c|}{$11 / 28 / 95$} & \multicolumn{2}{|c|}{$11 / 06 / 97$} \\
\hline Total count & \multicolumn{2}{|c|}{674} & \multicolumn{2}{|c|}{1,405} \\
\hline Total number of taxa & \multicolumn{2}{|c|}{27} & \multicolumn{2}{|c|}{31} \\
\hline Organism & Count & Percent & Count & Percent \\
\hline \multicolumn{5}{|l|}{ Trichoptera (CADDISFLIES) } \\
\hline \multicolumn{5}{|l|}{ Glossosomatidae } \\
\hline Glossosoma & - & & 6 & $0.43 \%$ \\
\hline \multicolumn{5}{|l|}{ Hydropsychidae } \\
\hline Ceratopsyche & 64 & $9.50 \%$ & 122 & $8.68 \%$ \\
\hline Cheumatopsyche & 3 & $.45 \%$ & 9 & $.64 \%$ \\
\hline Hydropsyche & 83 & $12.31 \%$ & 31 & $2.21 \%$ \\
\hline \multicolumn{5}{|l|}{ Hydroptilidae } \\
\hline Hydroptila & 4 & $.59 \%$ & 18 & $1.28 \%$ \\
\hline Leucotrichia & 24 & $3.56 \%$ & 255 & $18.15 \%$ \\
\hline \multicolumn{5}{|l|}{ Leptoceridae } \\
\hline Oecetis & - & & 2 & $.14 \%$ \\
\hline \multicolumn{5}{|l|}{ Philopotamidae } \\
\hline Chimarra & 23 & $3.41 \%$ & 140 & $9.96 \%$ \\
\hline \multicolumn{5}{|l|}{ Polycentropodidae } \\
\hline Neureclipsis & - & & 7 & $.50 \%$ \\
\hline Polycentropus & - & & 1 & $.07 \%$ \\
\hline \multicolumn{5}{|l|}{ Coleoptera (BEETLES) } \\
\hline \multicolumn{5}{|l|}{ Elmidae (RIFFLE BEETLES) } \\
\hline Optioservus & 13 & $1.93 \%$ & 6 & $.43 \%$ \\
\hline Stenelmis & 3 & $.45 \%$ & 3 & $.21 \%$ \\
\hline \multicolumn{5}{|l|}{ Hydrophilidae } \\
\hline Helophorus & 2 & $.30 \%$ & - & \\
\hline \multicolumn{5}{|c|}{ Psephenidae (WATER PENNIES) } \\
\hline Psephenus & 1 & $.15 \%$ & - & \\
\hline \multicolumn{5}{|l|}{ Diptera (TRUE FLIES) } \\
\hline Chironomidae (MIDGES) & 162 & $24.04 \%$ & 290 & $20.64 \%$ \\
\hline \multicolumn{5}{|l|}{ Empididae (DANCE FLIES) } \\
\hline Clinocera & - & & 2 & $.14 \%$ \\
\hline Hemerodromia & - & & 3 & $.21 \%$ \\
\hline \multicolumn{5}{|l|}{ Simuliidae (BLACK FLIES) } \\
\hline Simulium & 31 & $4.60 \%$ & 107 & $7.62 \%$ \\
\hline \multicolumn{5}{|l|}{ Tipulidae (CRANE FLIES) } \\
\hline Antocha & 90 & $13.35 \%$ & 115 & $8.19 \%$ \\
\hline Tipula & 1 & $.15 \%$ & 1 & $.07 \%$ \\
\hline
\end{tabular}


Table 5. Benthic-macroinvertebrate data-Continued

01480632 (Site 45) - Doe Run at Springdell, Pa.

\begin{tabular}{|c|c|c|c|c|}
\hline Date & \multicolumn{2}{|c|}{$11 / 28 / 95$} & \multicolumn{2}{|c|}{$11 / 06 / 97$} \\
\hline Total count & \multicolumn{2}{|c|}{820} & \multicolumn{2}{|c|}{1,078} \\
\hline Total number of taxa & \multicolumn{2}{|c|}{32} & \multicolumn{2}{|c|}{26} \\
\hline Organism & Count & Percent & Count & Percent \\
\hline \multicolumn{5}{|l|}{ Platyhelminthes } \\
\hline \multicolumn{5}{|l|}{ Turbellaria (FLATWORMS) } \\
\hline \multicolumn{5}{|l|}{ Tricladida } \\
\hline Planariidae & 12 & $1.46 \%$ & 10 & $0.93 \%$ \\
\hline Nematoda (NEMATODES) & 1 & $.12 \%$ & - & \\
\hline \multicolumn{5}{|l|}{ Mollusca } \\
\hline \multicolumn{5}{|l|}{ Gastropoda (SNAILS) } \\
\hline \multicolumn{5}{|l|}{ Basommatophora } \\
\hline \multicolumn{5}{|l|}{ Ancylidae } \\
\hline Ferrissia & 18 & $2.20 \%$ & 183 & $16.98 \%$ \\
\hline \multicolumn{5}{|l|}{ Annelida (SEGMENTED WORMS) } \\
\hline \multicolumn{5}{|l|}{ Oligochaeta } \\
\hline \multicolumn{5}{|l|}{ Tubificida } \\
\hline Naididae & 4 & $.49 \%$ & - & \\
\hline \multicolumn{5}{|l|}{ Arthropoda } \\
\hline \multicolumn{5}{|l|}{ Acariformes } \\
\hline Hydrachnidia (WATER MITES) & 6 & $.73 \%$ & 19 & $1.76 \%$ \\
\hline \multicolumn{5}{|l|}{ Insecta } \\
\hline \multicolumn{5}{|l|}{ Ephemeroptera (MAYFLIES) } \\
\hline Baetidae & & & & \\
\hline Baetis & 1 & $.12 \%$ & - & \\
\hline Pseudocloeon & 1 & $.12 \%$ & - & \\
\hline Ephemerellidae & & & & \\
\hline Ephemerella & 52 & $6.34 \%$ & 10 & $.93 \%$ \\
\hline Heptageniidae & & & & \\
\hline Epeorus & 41 & $5.00 \%$ & 44 & $4.08 \%$ \\
\hline Stenonema & 58 & $7.07 \%$ & 57 & $5.29 \%$ \\
\hline Isonychiidae & & & & \\
\hline Isonychia & 27 & $3.29 \%$ & 40 & $3.71 \%$ \\
\hline Plecoptera (STONEFLIES) & & & & \\
\hline Capniidae & & & & \\
\hline Allocapnia & 1 & $.12 \%$ & 1 & $.09 \%$ \\
\hline Chloroperlidae & 1 & $.12 \%$ & - & \\
\hline Taeniopterygidae & & & & \\
\hline Taeniopteryx & 9 & $1.10 \%$ & - & \\
\hline Trichoptera (CADDISFLIES) & & & & \\
\hline Brachycentridae & & & & \\
\hline Micrasema & 1 & $.12 \%$ & - & \\
\hline Glossosomatidae & & & & \\
\hline Glossosoma & 2 & $.24 \%$ & 25 & $2.32 \%$ \\
\hline Hydropsychidae & & & & \\
\hline Ceratopsyche & 76 & $9.27 \%$ & 152 & $14.10 \%$ \\
\hline Cheumatopsyche & 6 & $.73 \%$ & 17 & $1.58 \%$ \\
\hline Hydropsyche & 15 & $1.83 \%$ & 58 & $5.38 \%$ \\
\hline Hydroptilidae & & & & \\
\hline Hydroptila & 1 & $.12 \%$ & 19 & $1.76 \%$ \\
\hline Leucotrichia & 76 & $9.27 \%$ & 63 & $5.84 \%$ \\
\hline Philopotamidae & & & & \\
\hline Chimarra & 12 & $1.46 \%$ & 5 & $.46 \%$ \\
\hline
\end{tabular}


Table 5. Benthic-macroinvertebrate data-Continued

01480632 (Site 45) - Doe Run at Springdell, Pa.-Continued

\begin{tabular}{|c|c|c|c|c|}
\hline Date & \multicolumn{2}{|c|}{$11 / 28 / 95$} & \multicolumn{2}{|c|}{$11 / 06 / 97$} \\
\hline Total count & \multicolumn{2}{|c|}{820} & \multicolumn{2}{|c|}{1,078} \\
\hline Total number of taxa & \multicolumn{2}{|c|}{32} & \multicolumn{2}{|c|}{26} \\
\hline Organism & Count & Percent & Count & Percent \\
\hline \multicolumn{5}{|l|}{ Trichoptera (CADDISFLIES) } \\
\hline \multicolumn{5}{|l|}{ Polycentropodidae } \\
\hline Neureclipsis & 1 & $0.12 \%$ & 4 & $0.37 \%$ \\
\hline Polycentropus & - & & 2 & $.19 \%$ \\
\hline \multicolumn{5}{|l|}{ Psychomyiidae } \\
\hline Psychomyia & 10 & $1.22 \%$ & 63 & $5.84 \%$ \\
\hline \multicolumn{5}{|l|}{ Uenoidae } \\
\hline Neophylax & 10 & $1.22 \%$ & 1 & $.09 \%$ \\
\hline \multicolumn{5}{|l|}{ Lepidoptera } \\
\hline \multicolumn{5}{|l|}{ Pyralidae (MOTHS) } \\
\hline Petrophila & 3 & $.37 \%$ & 24 & $2.23 \%$ \\
\hline \multicolumn{5}{|l|}{ Coleoptera (BEETLES) } \\
\hline \multicolumn{5}{|l|}{ Elmidae (RIFFLE BEETLES) } \\
\hline Optioservus & 10 & $1.22 \%$ & 6 & $.56 \%$ \\
\hline Oulimnius & - & & 3 & $.28 \%$ \\
\hline Stenelmis & 1 & $.12 \%$ & - & \\
\hline \multicolumn{5}{|l|}{ Psephenidae (WATER PENNIES) } \\
\hline Psephenus & - & & 1 & $.09 \%$ \\
\hline \multicolumn{5}{|l|}{ Diptera (TRUE FLIES) } \\
\hline Chironomidae (MIDGES) & 207 & $25.24 \%$ & 125 & $11.60 \%$ \\
\hline \multicolumn{5}{|l|}{ Empididae (DANCE FLIES) } \\
\hline Hemerodromia & 8 & $.98 \%$ & - & \\
\hline \multicolumn{5}{|l|}{ Simuliidae (BLACK FLIES) } \\
\hline Simulium & 39 & $4.76 \%$ & 8 & $.74 \%$ \\
\hline \multicolumn{5}{|l|}{ Tipulidae (CRANE FLIES) } \\
\hline Antocha & 110 & $13.41 \%$ & 138 & $12.80 \%$ \\
\hline
\end{tabular}


Table 5. Benthic-macroinvertebrate data-Continued

01480640 (Site 38) - West Branch Brandywine Creek at Wawaset, Pa.

\begin{tabular}{|c|c|c|c|c|c|c|}
\hline Date & \multicolumn{2}{|c|}{$11 / 20 / 95$} & \multicolumn{2}{|c|}{$10 / 15 / 96$} & \multicolumn{2}{|c|}{$10 / 10 / 97$} \\
\hline Total count & \multicolumn{2}{|c|}{547} & \multicolumn{2}{|c|}{861} & \multicolumn{2}{|c|}{1,476} \\
\hline Total number of taxa & \multicolumn{2}{|c|}{43} & \multicolumn{2}{|c|}{30} & \multicolumn{2}{|c|}{38} \\
\hline Organism & Count & Percent & Count & Percent & Count & Percent \\
\hline \multicolumn{7}{|l|}{ Platyhelminthes } \\
\hline Planariidae & 28 & $5.12 \%$ & 14 & $1.63 \%$ & 65 & $4.40 \%$ \\
\hline Nematoda (NEMATODES) & - & & 1 & $.12 \%$ & 5 & $.34 \%$ \\
\hline \multicolumn{7}{|l|}{ Nemertea (PROBOSCIS WORMS) } \\
\hline \multicolumn{7}{|l|}{ Enopla } \\
\hline \multicolumn{7}{|l|}{ Hoplonemertea } \\
\hline \multicolumn{7}{|l|}{ Tetrastemmatidae } \\
\hline Prostoma & 2 & $.37 \%$ & 1 & $.12 \%$ & 1 & $.07 \%$ \\
\hline \multicolumn{7}{|l|}{ Mollusca } \\
\hline \multicolumn{7}{|l|}{ Gastropoda (SNAILS) } \\
\hline \multicolumn{7}{|l|}{ Basommatophora } \\
\hline \multicolumn{7}{|l|}{ Ancylidae } \\
\hline Ferrissia & 5 & $.91 \%$ & 26 & $3.02 \%$ & 30 & $2.03 \%$ \\
\hline Physidae & & & & & & \\
\hline Physa & 2 & $.37 \%$ & - & & - & \\
\hline Bivalvia (CLAMS) & & & & & & \\
\hline Veneroida & & & & & & \\
\hline Sphaeriidae & 8 & $1.46 \%$ & 3 & $.35 \%$ & 1 & $.07 \%$ \\
\hline Annelida (SEGMENTED WORMS) & & & & & & \\
\hline Oligochaeta & & & & & & \\
\hline Tubificida & & & & & & \\
\hline Naididae & 2 & $.37 \%$ & 1 & $.12 \%$ & - & \\
\hline Arthropoda & & & & & & \\
\hline Acariformes & & & & & & \\
\hline Hydrachnidia (WATER MITES) & 27 & $4.94 \%$ & 15 & $1.74 \%$ & 28 & $1.90 \%$ \\
\hline Crustacea & & & & & & \\
\hline Cyclopoida & & & & & & \\
\hline Cyclopidae & 1 & $.18 \%$ & - & & - & \\
\hline Amphipoda (SCUDS) & & & & & & \\
\hline Gammaridae & & & & & & \\
\hline Gammarus & 5 & $.91 \%$ & - & & 3 & $.20 \%$ \\
\hline Isopoda (SOW BUGS) & & & & & & \\
\hline Asellidae & 1 & $.18 \%$ & - & & - & \\
\hline Insecta & & & & & & \\
\hline Ephemeroptera (MAYFLIES) & & & & & & \\
\hline Baetidae & & & & & & \\
\hline Baetis & 1 & $.18 \%$ & - & & 45 & $3.05 \%$ \\
\hline Pseudocloeon & - & & 7 & $.81 \%$ & 23 & $1.56 \%$ \\
\hline Caenidae & & & & & & \\
\hline $\begin{array}{c}\text { Caenis } \\
\text { Ephemerellidae }\end{array}$ & 21 & $3.84 \%$ & 2 & $.23 \%$ & 1 & $.07 \%$ \\
\hline $\begin{array}{l}\text { Ephemerella } \\
\text { Heptageniidae }\end{array}$ & 43 & $7.86 \%$ & 55 & $6.39 \%$ & 109 & $7.38 \%$ \\
\hline Epeorus & - & & 2 & $.23 \%$ & 1 & $.07 \%$ \\
\hline Stenonema & 41 & $7.50 \%$ & 43 & $4.99 \%$ & 58 & $3.93 \%$ \\
\hline Isonychiidae & & & & & & \\
\hline $\begin{array}{r}\text { Isonychia } \\
\text { Leptohyphidae }\end{array}$ & 4 & $.73 \%$ & 8 & $.93 \%$ & 4 & $.27 \%$ \\
\hline Paraleptophlebia & 2 & $.37 \%$ & - & & - & \\
\hline Tricorythodes & 8 & $1.46 \%$ & - & & 4 & $.27 \%$ \\
\hline
\end{tabular}


Table 5. Benthic-macroinvertebrate data-Continued

01480640 (Site 38) - West Branch Brandywine Creek at Wawaset, Pa.-Continued

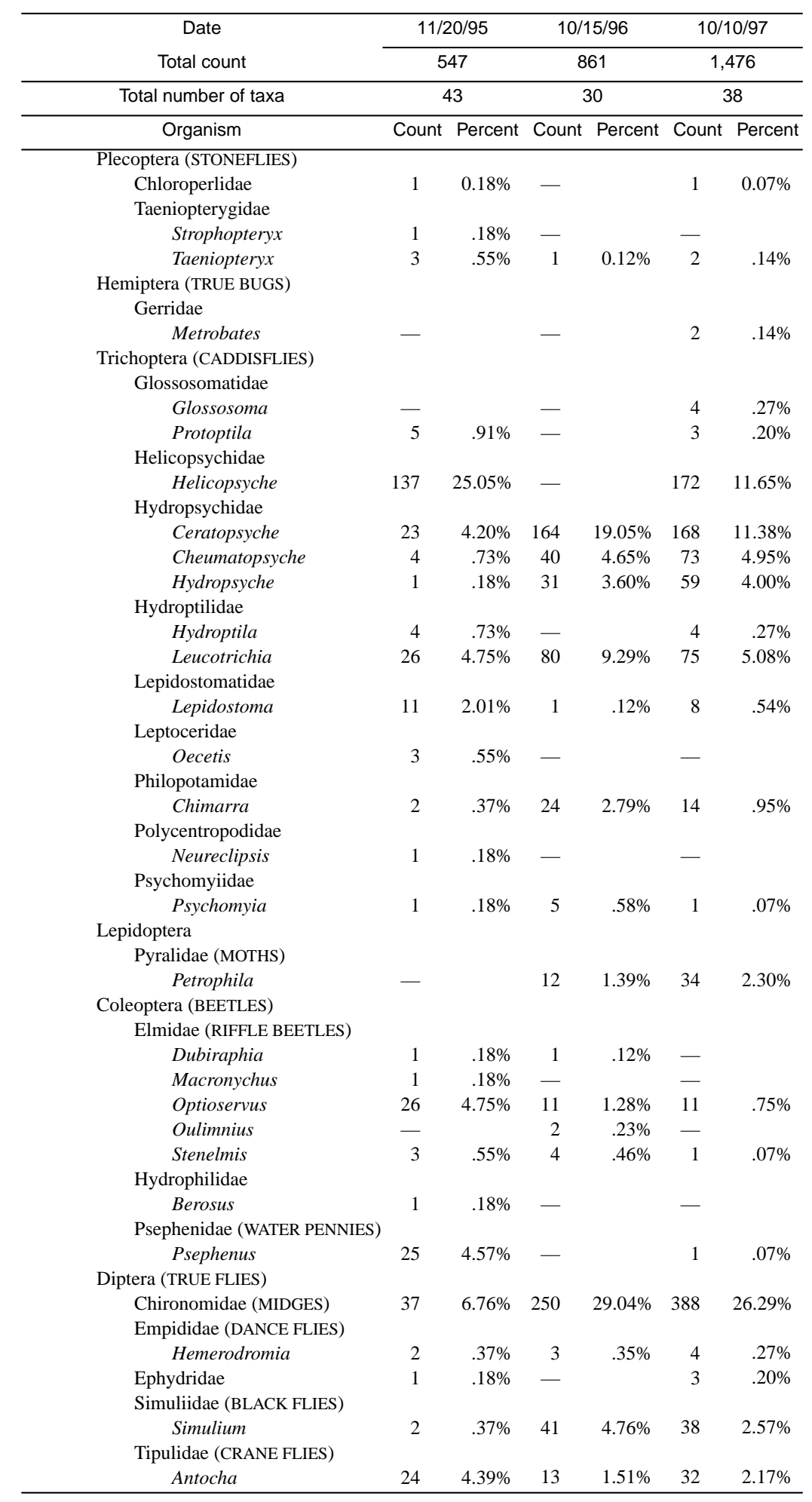


Table 5. Benthic-macroinvertebrate data-Continued

01480648 (Site 48) - East Branch Brandywine Creek near Cupola, Pa.

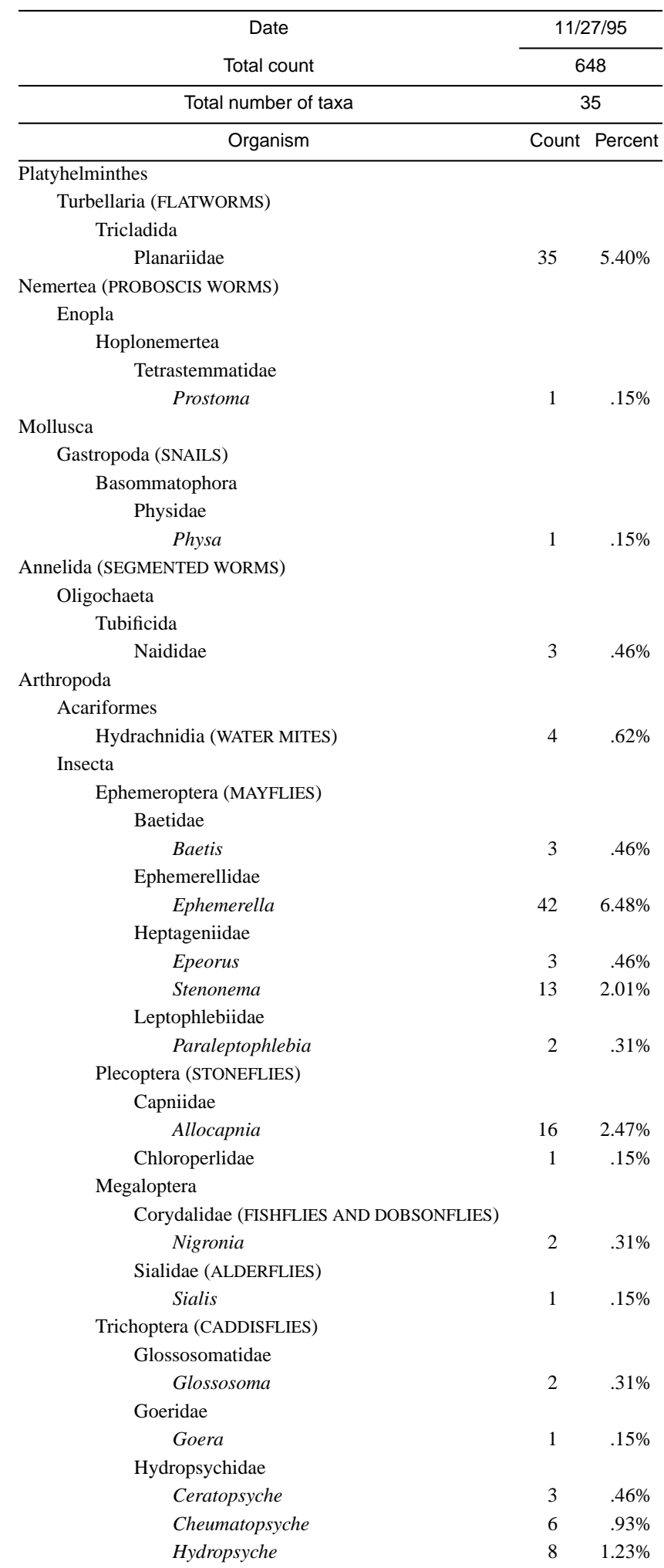


Table 5. Benthic-macroinvertebrate data-Continued

01480648 (Site 48) - East Branch Brandywine Creek near Cupola, Pa.-Continued

\begin{tabular}{|c|c|c|}
\hline Date & \multirow{2}{*}{\multicolumn{2}{|c|}{$\frac{11 / 27 / 95}{648}$}} \\
\hline Total count & & \\
\hline Total number of taxa & \multicolumn{2}{|c|}{35} \\
\hline Organism & Count & Percent \\
\hline \multicolumn{3}{|l|}{ Trichoptera (CADDISFLIES) } \\
\hline \multicolumn{3}{|l|}{ Hydroptilidae } \\
\hline Hydroptila & 1 & $0.15 \%$ \\
\hline \multicolumn{3}{|l|}{ Lepidostomatidae } \\
\hline Lepidostoma & 1 & $.15 \%$ \\
\hline \multicolumn{3}{|l|}{ Leptoceridae } \\
\hline Mystacides & 1 & $.15 \%$ \\
\hline Oecetis & 1 & $.15 \%$ \\
\hline \multicolumn{3}{|l|}{ Odontoceridae } \\
\hline Psilotreta & 3 & $.46 \%$ \\
\hline \multicolumn{3}{|l|}{ Philopotamidae } \\
\hline Chimarra & 64 & $9.88 \%$ \\
\hline \multicolumn{3}{|l|}{ Polycentropodidae } \\
\hline Polycentropus & 1 & $.15 \%$ \\
\hline \multicolumn{3}{|l|}{ Uenoidae } \\
\hline Neophylax & 15 & $2.31 \%$ \\
\hline \multicolumn{3}{|l|}{ Coleoptera (BEETLES) } \\
\hline \multicolumn{3}{|l|}{ Elmidae (RIFFLE BEETLES) } \\
\hline Dubiraphia & 1 & $.15 \%$ \\
\hline Optioservus & 96 & $14.81 \%$ \\
\hline Oulimnius & 7 & $1.08 \%$ \\
\hline Stenelmis & 67 & $10.34 \%$ \\
\hline \multicolumn{3}{|c|}{ Psephenidae (WATER PENNIES) } \\
\hline Psephenus & 49 & $7.56 \%$ \\
\hline \multicolumn{3}{|l|}{ Diptera (TRUE FLIES) } \\
\hline Chironomidae (MIDGES) & 63 & $9.72 \%$ \\
\hline \multicolumn{3}{|l|}{ Simuliidae (BLACK FLIES) } \\
\hline Simulium & 114 & $17.59 \%$ \\
\hline \multicolumn{3}{|l|}{ Tipulidae (CRANE FLIES) } \\
\hline Antocha & 17 & $2.62 \%$ \\
\hline
\end{tabular}


Table 5. Benthic-macroinvertebrate data-Continued

01480653 (Site 42) - East Branch Brandywine Creek at Glenmoore, Pa.

\begin{tabular}{|c|c|c|}
\hline Date & \multicolumn{2}{|c|}{$12 / 01 / 95$} \\
\hline Total count & \multicolumn{2}{|c|}{625} \\
\hline Total number of taxa & \multicolumn{2}{|c|}{34} \\
\hline Organism & Count & Percent \\
\hline \multicolumn{3}{|l|}{ Platyhelminthes } \\
\hline \multicolumn{3}{|l|}{ Turbellaria (FLATWORMS) } \\
\hline \multicolumn{3}{|l|}{ Tricladida } \\
\hline Planariidae & 7 & $1.12 \%$ \\
\hline \multicolumn{3}{|l|}{ Nemertea (PROBOSCIS WORMS) } \\
\hline \multicolumn{3}{|l|}{ Enopla } \\
\hline \multicolumn{3}{|l|}{ Hoplonemertea } \\
\hline \multicolumn{3}{|l|}{ Tetrastemmatidae } \\
\hline Prostoma & 2 & $.32 \%$ \\
\hline \multicolumn{3}{|l|}{ Mollusca } \\
\hline \multicolumn{3}{|l|}{ Gastropoda (SNAILS) } \\
\hline \multicolumn{3}{|l|}{ Basommatophora } \\
\hline \multicolumn{3}{|l|}{ Ancylidae } \\
\hline Ferrissia & 3 & $.48 \%$ \\
\hline \multicolumn{3}{|l|}{ Arthropoda } \\
\hline \multicolumn{3}{|l|}{ Acariformes } \\
\hline Hydrachnidia (WATER MITES) & 127 & $2.32 \%$ \\
\hline \multicolumn{3}{|l|}{ Insecta } \\
\hline \multicolumn{3}{|l|}{ Ephemeroptera (MAYFLIES) } \\
\hline \multicolumn{3}{|l|}{ Baetidae } \\
\hline Pseudocloeon & 1 & $.16 \%$ \\
\hline \multicolumn{3}{|l|}{ Ephemerellidae } \\
\hline Ephemerella & 44 & $7.04 \%$ \\
\hline Heptageniidae & & \\
\hline Epeorus & 3 & $.48 \%$ \\
\hline Stenonema & 8 & $1.28 \%$ \\
\hline Isonychiidae & & \\
\hline Isonychia & 1 & $.16 \%$ \\
\hline Plecoptera (STONEFLIES) & & \\
\hline Capniidae & & \\
\hline Allocapnia & 2 & $.32 \%$ \\
\hline Taeniopterygidae & & \\
\hline Strophopteryx & 34 & $5.44 \%$ \\
\hline Taeniopteryx & 1 & $.16 \%$ \\
\hline Trichoptera (CADDISFLIES) & & \\
\hline Apataniidae & & \\
\hline Apatania & 5 & $.80 \%$ \\
\hline Brachycentridae & & \\
\hline Micrasema & 21 & $3.36 \%$ \\
\hline Glossosomatidae & & \\
\hline Glossosoma & 3 & $.48 \%$ \\
\hline Goeridae & & \\
\hline Goera & 3 & $.48 \%$ \\
\hline Hydropsychidae & & \\
\hline Ceratopsyche & 11 & $1.76 \%$ \\
\hline Cheumatopsyche & 29 & $4.64 \%$ \\
\hline Hydropsyche & 12 & $1.92 \%$ \\
\hline Hydroptilidae & & \\
\hline Hydroptila & 1 & $.16 \%$ \\
\hline Leptoceridae & & \\
\hline Oecetis & 2 & $.32 \%$ \\
\hline
\end{tabular}


Table 5. Benthic-macroinvertebrate data-Continued

01480653 (Site 42) - East Branch Brandywine Creek at Glenmoore, Pa.-Continued

\begin{tabular}{|c|c|c|}
\hline Date & \multicolumn{2}{|c|}{$12 / 01 / 95$} \\
\hline Total count & \multicolumn{2}{|c|}{625} \\
\hline Total number of taxa & \multicolumn{2}{|c|}{34} \\
\hline Organism & Count & Percent \\
\hline \multicolumn{3}{|l|}{ Trichoptera (CADDISFLIES) } \\
\hline \multicolumn{3}{|l|}{ Philopotamidae } \\
\hline Chimarra & 9 & $1.44 \%$ \\
\hline \multicolumn{3}{|l|}{ Coleoptera (BEETLES) } \\
\hline \multicolumn{3}{|l|}{ Elmidae (RIFFLE BEETLES) } \\
\hline Ancyronyx & 2 & $.32 \%$ \\
\hline Dubiraphia & 2 & $.32 \%$ \\
\hline Optioservus & 18 & $2.88 \%$ \\
\hline Promoresia & 3 & $.48 \%$ \\
\hline Stenelmis & 9 & $1.44 \%$ \\
\hline \multicolumn{3}{|c|}{ Psephenidae (WATER PENNIES) } \\
\hline Ectopria & 1 & $.16 \%$ \\
\hline Psephenus & 6 & $.96 \%$ \\
\hline \multicolumn{3}{|l|}{ Diptera (TRUE FLIES) } \\
\hline Chironomidae (MIDGES) & 140 & $22.40 \%$ \\
\hline \multicolumn{3}{|l|}{ Empididae (DANCE FLIES) } \\
\hline Chelifera & 3 & $.48 \%$ \\
\hline Hemerodromia & 1 & $.16 \%$ \\
\hline \multicolumn{3}{|l|}{ Simuliidae (BLACK FLIES) } \\
\hline Simulium & 97 & $15.52 \%$ \\
\hline \multicolumn{3}{|l|}{ Tipulidae (CRANE FLIES) } \\
\hline Antocha & 14 & $2.24 \%$ \\
\hline
\end{tabular}


Table 5. Benthic-macroinvertebrate data-Continued

01480656 (Site 47) - Indian Run near Springton, Pa.

\begin{tabular}{|c|c|c|}
\hline Date & \multirow{2}{*}{\multicolumn{2}{|c|}{$\frac{11 / 27 / 95}{647}$}} \\
\hline Total count & & \\
\hline Total number of taxa & \multicolumn{2}{|c|}{33} \\
\hline Organism & Count & Percent \\
\hline \multicolumn{3}{|l|}{ Mollusca } \\
\hline \multicolumn{3}{|l|}{ Gastropoda (SNAILS) } \\
\hline \multicolumn{3}{|l|}{ Basommatophora } \\
\hline \multicolumn{3}{|l|}{ Ancylidae } \\
\hline Ferrissia & 6 & $0.93 \%$ \\
\hline \multicolumn{3}{|l|}{ Annelida (SEGMENTED WORMS) } \\
\hline \multicolumn{3}{|l|}{ Oligochaeta } \\
\hline \multicolumn{3}{|l|}{ Lumbriculida } \\
\hline Lumbriculidae & 1 & $.15 \%$ \\
\hline \multicolumn{3}{|l|}{ Tubificida } \\
\hline Naididae & 11 & $1.70 \%$ \\
\hline \multicolumn{3}{|l|}{ Arthropoda } \\
\hline \multicolumn{3}{|l|}{ Acariformes } \\
\hline Hydrachnidia (WATER MITES) & 14 & $2.16 \%$ \\
\hline \multicolumn{3}{|l|}{ Insecta } \\
\hline \multicolumn{3}{|l|}{ Ephemeroptera (MAYFLIES) } \\
\hline \multicolumn{3}{|l|}{ Ephemerellidae } \\
\hline Ephemerella & 71 & $10.97 \%$ \\
\hline \multicolumn{3}{|l|}{ Heptageniidae } \\
\hline Epeorus & 1 & $.15 \%$ \\
\hline Stenonema & 2 & $.31 \%$ \\
\hline \multicolumn{3}{|c|}{ Odonata (DRAGONFLIES AND DAMSELFLIES) } \\
\hline Gomphidae & 1 & $.15 \%$ \\
\hline \multicolumn{3}{|l|}{ Plecoptera (STONEFLIES) } \\
\hline \multicolumn{3}{|l|}{ Capniidae } \\
\hline Allocapnia & 5 & $.77 \%$ \\
\hline Chloroperlidae & 1 & $.15 \%$ \\
\hline Taeniopterygidae & & \\
\hline Strophopteryx & 87 & $13.45 \%$ \\
\hline Taeniopteryx & 1 & $.15 \%$ \\
\hline Megaloptera & & \\
\hline Corydalidae (FISHFLIES ANI & & \\
\hline Nigronia & 1 & $.15 \%$ \\
\hline Trichoptera (CADDISFLIES) & & \\
\hline Apataniidae & & \\
\hline Apatania & 20 & $3.09 \%$ \\
\hline Glossosomatidae & & \\
\hline Glossosoma & 19 & $2.94 \%$ \\
\hline Goeridae & & \\
\hline Goera & 2 & $.31 \%$ \\
\hline Hydropsychidae & & \\
\hline Ceratopsyche & 29 & $4.48 \%$ \\
\hline Cheumatopsyche & 19 & $2.94 \%$ \\
\hline Diplectrona & 5 & $.77 \%$ \\
\hline Hydropsyche & 4 & $.62 \%$ \\
\hline Leptoceridae & & \\
\hline Mystacides & 2 & $.31 \%$ \\
\hline Philopotamidae & & \\
\hline Chimarra & 2 & $.31 \%$ \\
\hline Psychomyiidae & & \\
\hline Psychomyia & 5 & $.77 \%$ \\
\hline
\end{tabular}

Trichoptera (CADDISFLIES) 
Table 5. Benthic-macroinvertebrate data-Continued

01480656 (Site 47) - Indian Run near Springton, Pa.-Continued

\begin{tabular}{|c|c|c|}
\hline Date & \multicolumn{2}{|c|}{$11 / 27 / 95$} \\
\hline Total count & \multicolumn{2}{|c|}{647} \\
\hline Total number of taxa & \multicolumn{2}{|c|}{33} \\
\hline Organism & Count & Percent \\
\hline \multicolumn{3}{|l|}{ Rhyacophilidae } \\
\hline Rhyacophila & 5 & $0.77 \%$ \\
\hline \multicolumn{3}{|l|}{ Uenoidae } \\
\hline Neophylax & 10 & $1.55 \%$ \\
\hline \multicolumn{3}{|l|}{ Coleoptera (BEETLES) } \\
\hline \multicolumn{3}{|l|}{ Elmidae (RIFFLE BEETLES) } \\
\hline Optioservus & 3 & $.46 \%$ \\
\hline Promoresia & 1 & $.15 \%$ \\
\hline Stenelmis & 1 & $.15 \%$ \\
\hline \multicolumn{3}{|l|}{ Psephenidae (WATER PENNIES) } \\
\hline Ectopria & 5 & $.77 \%$ \\
\hline Psephenus & 2 & $.31 \%$ \\
\hline \multicolumn{3}{|l|}{ Diptera (TRUE FLIES) } \\
\hline Chironomidae (MIDGES) & 98 & $15.15 \%$ \\
\hline \multicolumn{3}{|l|}{ Simuliidae (BLACK FLIES) } \\
\hline Simulium & 185 & $28.59 \%$ \\
\hline \multicolumn{3}{|l|}{ Tipulidae (CRANE FLIES) } \\
\hline Antocha & 28 & $4.33 \%$ \\
\hline
\end{tabular}


Table 5. Benthic-macroinvertebrate data-Continued

01480700 (Site 36) - East Branch Brandywine Creek near Downingtown, Pa.

\begin{tabular}{|c|c|c|c|c|}
\hline Date & \multicolumn{2}{|c|}{$10 / 17 / 95$} & \multicolumn{2}{|c|}{$10 / 29 / 96$} \\
\hline Total count & \multicolumn{2}{|c|}{1,508} & \multicolumn{2}{|c|}{1,347} \\
\hline Total number of taxa & \multicolumn{2}{|c|}{41} & \multicolumn{2}{|c|}{49} \\
\hline Organism & Count & Percent & Count & Percent \\
\hline \multicolumn{5}{|l|}{ Platyhelminthes } \\
\hline \multicolumn{5}{|l|}{ Turbellaria (FLATWORMS) } \\
\hline \multicolumn{5}{|l|}{ Tricladida } \\
\hline Planariidae & 5 & $0.33 \%$ & 18 & $1.34 \%$ \\
\hline Nematoda (NEMATODES) & - & & 1 & $.07 \%$ \\
\hline \multicolumn{5}{|l|}{ Nemertea (PROBOSCIS WORMS) } \\
\hline \multicolumn{5}{|l|}{ Enopla } \\
\hline \multicolumn{5}{|l|}{ Hoplonemertea } \\
\hline \multicolumn{5}{|l|}{ Tetrastemmatidae } \\
\hline Prostoma & - & & 1 & $.07 \%$ \\
\hline \multicolumn{5}{|l|}{ Mollusca } \\
\hline \multicolumn{5}{|l|}{ Gastropoda (SNAILS) } \\
\hline \multicolumn{5}{|l|}{ Basommatophora } \\
\hline \multicolumn{5}{|l|}{ Ancylidae } \\
\hline Ferrissia & 6 & $.40 \%$ & 3 & $.22 \%$ \\
\hline \multicolumn{5}{|l|}{ Annelida (SEGMENTED WORMS) } \\
\hline Oligochaeta & & & & \\
\hline Tubificida & & & & \\
\hline Naididae & 16 & $1.06 \%$ & 9 & $.67 \%$ \\
\hline Arthropoda & & & & \\
\hline Acariformes & & & & \\
\hline Hydrachnidia (WATER MITES) & 66 & $4.38 \%$ & 104 & $7.72 \%$ \\
\hline Crustacea & & & & \\
\hline Cladocera & - & & 121 & $8.98 \%$ \\
\hline Cyclopoida & - & & 5 & $.37 \%$ \\
\hline Insecta & & & & \\
\hline Ephemeroptera (MAYFLIES) & & & & \\
\hline Baetidae & & & & \\
\hline Pseudocloeon & 9 & $.60 \%$ & 1 & $.07 \%$ \\
\hline Caenidae & & & & \\
\hline Caenis & 2 & $.13 \%$ & 1 & $.07 \%$ \\
\hline Ephemerellidae & & & & \\
\hline Ephemerella & 43 & $2.85 \%$ & 60 & $4.45 \%$ \\
\hline Heptageniidae & & & & \\
\hline Epeorus & 3 & $.20 \%$ & 8 & $.59 \%$ \\
\hline Stenonema & 34 & $2.25 \%$ & 6 & $.45 \%$ \\
\hline Isonychiidae & & & & \\
\hline Isonychia & 12 & $.80 \%$ & 5 & $.37 \%$ \\
\hline Odonata (DRAGONFLIES AND D & & & & \\
\hline Coenagrionidae & & & & \\
\hline Argia & - & & 1 & $.07 \%$ \\
\hline Plecoptera (STONEFLIES) & & & & \\
\hline Capniidae & & & & \\
\hline Allocapnia & - & & 13 & $.97 \%$ \\
\hline Chloroperlidae & 2 & $.13 \%$ & 11 & $.82 \%$ \\
\hline Perlidae & & & & \\
\hline Acroneuria & 2 & $.13 \%$ & 1 & $.07 \%$ \\
\hline Agnetina & 3 & $.20 \%$ & 5 & $.37 \%$ \\
\hline Taeniopterygidae & & & & \\
\hline Taeniopteryx & 3 & $.20 \%$ & 45 & $3.34 \%$ \\
\hline Megaloptera & & & & \\
\hline Corydalidae (FISHFLIES AN & & & & \\
\hline Corydalus & 1 & $.07 \%$ & 1 & $.07 \%$ \\
\hline
\end{tabular}


Table 5. Benthic-macroinvertebrate data-Continued

01480700 (Site 36) - East Branch Brandywine Creek near Downingtown, Pa.-Continued

\begin{tabular}{|c|c|c|c|c|}
\hline Date & \multicolumn{2}{|c|}{$10 / 17 / 95$} & \multicolumn{2}{|c|}{$10 / 29 / 96$} \\
\hline Total count & \multicolumn{2}{|c|}{1,508} & \multicolumn{2}{|c|}{1,347} \\
\hline Total number of taxa & \multicolumn{2}{|c|}{41} & \multicolumn{2}{|c|}{49} \\
\hline Organism & Count & Percent & Count & Percent \\
\hline \multicolumn{5}{|l|}{ Trichoptera (CADDISFLIES) } \\
\hline \multicolumn{5}{|l|}{ Apataniidae } \\
\hline Apatania & 10 & $0.66 \%$ & 23 & $1.71 \%$ \\
\hline \multicolumn{5}{|l|}{ Brachycentridae } \\
\hline Micrasema & 84 & $5.57 \%$ & 80 & $5.94 \%$ \\
\hline \multicolumn{5}{|l|}{ Glossosomatidae } \\
\hline Glossosoma & 5 & $.33 \%$ & 14 & $1.04 \%$ \\
\hline \multicolumn{5}{|l|}{ Goeridae } \\
\hline \multirow{2}{*}{\multicolumn{5}{|c|}{ Helicopsychidae }} \\
\hline & & & & \\
\hline Helicopsyche & 9 & $.60 \%$ & 3 & $.22 \%$ \\
\hline \multicolumn{5}{|l|}{ Hydropsychidae } \\
\hline Ceratopsyche & 476 & $31.56 \%$ & 261 & $19.38 \%$ \\
\hline Cheumatopsyche & 79 & $5.24 \%$ & 49 & $3.64 \%$ \\
\hline Hydropsyche & 76 & $5.04 \%$ & 25 & $1.86 \%$ \\
\hline \multicolumn{5}{|l|}{ Hydroptilidae } \\
\hline Hydroptila & 1 & $.07 \%$ & - & \\
\hline Leucotrichia & 101 & $6.70 \%$ & 3 & $.22 \%$ \\
\hline \multicolumn{5}{|l|}{ Lepidostomatidae } \\
\hline Lepidostoma & 1 & $.07 \%$ & 11 & $.82 \%$ \\
\hline \multicolumn{5}{|l|}{ Leptoceridae } \\
\hline Oecetis & 2 & $.13 \%$ & 2 & $.15 \%$ \\
\hline \multicolumn{5}{|l|}{ Philopotamidae } \\
\hline Chimarra & 7 & $.46 \%$ & 1 & $.07 \%$ \\
\hline Dolophilodes & - & & 1 & $.07 \%$ \\
\hline Polycentropodidae & & & & \\
\hline Neureclipsis & 2 & $.13 \%$ & - & \\
\hline Nyctiophylax & 3 & $.20 \%$ & - & \\
\hline Polycentropus & - & & 1 & $.07 \%$ \\
\hline Psychomyiidae & & & & \\
\hline Psychomyia & 20 & $1.33 \%$ & 22 & $1.63 \%$ \\
\hline Rhyacophilidae & & & & \\
\hline Rhyacophila & 3 & $.20 \%$ & 7 & $.52 \%$ \\
\hline Lepidoptera & & & & \\
\hline Pyralidae (MOTHS) & & & & \\
\hline Petrophila & 12 & $.80 \%$ & 8 & $.59 \%$ \\
\hline Coleoptera (BEETLES) & & & & \\
\hline Elmidae (RIFFLE BEETLES) & & & & \\
\hline Dubiraphia & - & & 5 & $.37 \%$ \\
\hline Optioservus & 21 & $1.39 \%$ & 63 & $4.68 \%$ \\
\hline Oulimnius & 1 & $.07 \%$ & - & \\
\hline Promoresia & 65 & $4.31 \%$ & 41 & $3.04 \%$ \\
\hline Stenelmis & 5 & $.33 \%$ & 3 & $.22 \%$ \\
\hline Psephenidae (WATER PENN & & & & \\
\hline Psephenus & 6 & $.40 \%$ & 4 & $.30 \%$ \\
\hline Diptera (TRUE FLIES) & & & & \\
\hline Ceratopogonidae & - & & 1 & $.07 \%$ \\
\hline Chironomidae (MIDGES) & 257 & $17.04 \%$ & 230 & $17.07 \%$ \\
\hline Empididae (DANCE FLIES) & & & & \\
\hline Hemerodromia & 7 & $.46 \%$ & 17 & $1.26 \%$ \\
\hline Ephydridae & - & & 1 & $.07 \%$ \\
\hline Simuliidae (BLACK FLIES) & & & & \\
\hline Simulium & 2 & $.13 \%$ & 24 & $1.78 \%$ \\
\hline Tipulidae (CRANE FLIES) & & & & \\
\hline Antocha & 46 & $3.05 \%$ & 25 & $1.86 \%$ \\
\hline
\end{tabular}


Table 5. Benthic-macroinvertebrate data-Continued

01480903 (Site 44) - Valley Creek at Mullsteins Meadows near Downingtown, Pa.

\begin{tabular}{|c|c|c|c|c|}
\hline Date & \multicolumn{2}{|c|}{$11 / 21 / 95$} & \multicolumn{2}{|c|}{$11 / 04 / 97$} \\
\hline Total count & \multicolumn{2}{|c|}{1,327} & \multicolumn{2}{|c|}{3,137} \\
\hline Total number of taxa & \multicolumn{2}{|c|}{34} & \multicolumn{2}{|c|}{36} \\
\hline Organism & Count & Percent & Count & Percent \\
\hline \multicolumn{5}{|l|}{ Platyhelminthes } \\
\hline \multicolumn{5}{|l|}{ Turbellaria (FLATWORMS) } \\
\hline \multicolumn{5}{|l|}{ Tricladida } \\
\hline Planariidae & 63 & $4.75 \%$ & 146 & $4.65 \%$ \\
\hline Nematoda (NEMATODES) & - & & 1 & $.03 \%$ \\
\hline \multicolumn{5}{|l|}{ Mollusca } \\
\hline \multicolumn{5}{|l|}{ Gastropoda (SNAILS) } \\
\hline \multicolumn{5}{|l|}{ Basommatophora } \\
\hline \multicolumn{5}{|l|}{ Planorbidae } \\
\hline Gyraulus & - & & 2 & $.06 \%$ \\
\hline \multicolumn{5}{|l|}{ Bivalvia (CLAMS) } \\
\hline \multicolumn{5}{|l|}{ Veneroida } \\
\hline \multicolumn{5}{|l|}{ Sphaeriidae } \\
\hline Pisidium & 1 & $.08 \%$ & - & \\
\hline \multicolumn{5}{|l|}{ Annelida (SEGMENTED WORMS) } \\
\hline \multicolumn{5}{|l|}{ Oligochaeta } \\
\hline \multicolumn{5}{|l|}{ Tubificida } \\
\hline Naididae & 8 & $.60 \%$ & 1 & $.03 \%$ \\
\hline \multicolumn{5}{|l|}{ Arthropoda } \\
\hline Acariformes & & & & \\
\hline Hydrachnidia (WATER MITES) & 54 & $4.07 \%$ & 110 & $3.51 \%$ \\
\hline Crustacea & & & & \\
\hline Amphipoda (SCUDS) & & & & \\
\hline Gammaridae & & & & \\
\hline Gammarus & 2 & $.15 \%$ & 5 & $.16 \%$ \\
\hline Insecta & & & & \\
\hline Ephemeroptera (MAYFLIES) & & & & \\
\hline Baetidae & & & & \\
\hline Baetis & 1 & $.08 \%$ & 1 & $.03 \%$ \\
\hline Pseudocloeon & - & & 1 & $.03 \%$ \\
\hline Ephemerellidae & & & & \\
\hline Ephemerella & 196 & $14.77 \%$ & 220 & $7.01 \%$ \\
\hline Heptageniidae & & & & \\
\hline Stenonema & 8 & $.60 \%$ & 21 & $.67 \%$ \\
\hline Isonychiidae & & & & \\
\hline Isonychia & 3 & $.23 \%$ & 24 & $.77 \%$ \\
\hline Leptohyphidae & & & & \\
\hline Tricorythodes & 13 & $.98 \%$ & 15 & $.48 \%$ \\
\hline Odonata (DRAGONFLIES AND D & & & & \\
\hline Coenagrionidae & & & & \\
\hline Argia & - & & 1 & $.03 \%$ \\
\hline Plecoptera (STONEFLIES) & & & & \\
\hline Capniidae & & & & \\
\hline Allocapnia & 5 & $.38 \%$ & 3 & $.10 \%$ \\
\hline Chloroperlidae & 7 & $.53 \%$ & - & \\
\hline Perlidae & & & & \\
\hline Agnetina & - & & 4 & $.13 \%$ \\
\hline Taeniopterygidae & & & & \\
\hline Taeniopteryx & 3 & $.23 \%$ & 5 & $.16 \%$ \\
\hline
\end{tabular}

Hemiptera (TRUE BUGS) 
Table 5. Benthic-macroinvertebrate data-Continued

01480903 (Site 44) - Valley Creek at Mullsteins Meadows near Downingtown, Pa.-Continued

\begin{tabular}{|c|c|c|c|c|}
\hline Date & \multicolumn{2}{|c|}{ 11/21/95 } & \multicolumn{2}{|c|}{$11 / 04 / 97$} \\
\hline Total count & \multicolumn{2}{|c|}{1,327} & \multicolumn{2}{|c|}{3,137} \\
\hline Total number of taxa & \multicolumn{2}{|c|}{34} & \multicolumn{2}{|c|}{36} \\
\hline Organism & Count & Percent & Count & Percent \\
\hline \multicolumn{5}{|l|}{ Corixidae } \\
\hline Sigara & - & & 1 & $0.03 \%$ \\
\hline \multicolumn{5}{|l|}{ Trichoptera (CADDISFLIES) } \\
\hline \multicolumn{5}{|l|}{ Brachycentridae } \\
\hline Micrasema & 2 & $0.15 \%$ & 3 & $.10 \%$ \\
\hline \multicolumn{5}{|l|}{ Glossosomatidae } \\
\hline Glossosoma & - & & 2 & $.06 \%$ \\
\hline \multicolumn{5}{|l|}{ Helicopsychidae } \\
\hline Helicopsyche & 67 & $5.05 \%$ & 58 & $1.85 \%$ \\
\hline \multicolumn{5}{|l|}{ Hydropsychidae } \\
\hline Ceratopsyche & 241 & $18.16 \%$ & 638 & $20.34 \%$ \\
\hline Cheumatopsyche & 15 & $1.13 \%$ & 314 & $10.01 \%$ \\
\hline Hydropsyche & 18 & $1.36 \%$ & 61 & $1.94 \%$ \\
\hline \multicolumn{5}{|l|}{ Hydroptilidae } \\
\hline Hydroptila & 11 & $.83 \%$ & 36 & $1.15 \%$ \\
\hline Leucotrichia & 15 & $1.13 \%$ & 4 & $.13 \%$ \\
\hline \multicolumn{5}{|l|}{ Lepidostomatidae } \\
\hline Lepidostoma & 2 & $.15 \%$ & - & \\
\hline \multicolumn{5}{|l|}{ Leptoceridae } \\
\hline Mystacides & - & & 1 & $.03 \%$ \\
\hline Oecetis & 1 & $.08 \%$ & - & \\
\hline \multicolumn{5}{|l|}{ Philopotamidae } \\
\hline Chimarra & 7 & $.53 \%$ & 114 & $3.63 \%$ \\
\hline \multicolumn{5}{|l|}{ Psychomyiidae } \\
\hline Psychomyia & 6 & $.45 \%$ & 1 & $.03 \%$ \\
\hline \multicolumn{5}{|l|}{ Lepidoptera } \\
\hline \multicolumn{5}{|l|}{ Pyralidae (MOTHS) } \\
\hline Petrophila & 1 & $.08 \%$ & - & \\
\hline \multicolumn{5}{|l|}{ Coleoptera (BEETLES) } \\
\hline \multicolumn{5}{|l|}{ Elmidae (RIFFLE BEETLES) } \\
\hline Dubiraphia & 3 & $.23 \%$ & - & \\
\hline Optioservus & 110 & $8.29 \%$ & 99 & $3.16 \%$ \\
\hline Oulimnius & - & & 4 & $.13 \%$ \\
\hline Stenelmis & 41 & $3.09 \%$ & 60 & $1.91 \%$ \\
\hline \multicolumn{5}{|c|}{ Psephenidae (WATER PENNIES) } \\
\hline Ectopria & 1 & $.08 \%$ & - & \\
\hline Psephenus & 5 & $.38 \%$ & 3 & $.10 \%$ \\
\hline Diptera (TRUE FLIES) & & & & \\
\hline Chironomidae (MIDGES) & 244 & $18.39 \%$ & 950 & $30.28 \%$ \\
\hline Empididae (DANCE FLIES) & & & & \\
\hline Hemerodromia & 8 & $.60 \%$ & 21 & $.67 \%$ \\
\hline Simuliidae (BLACK FLIES) & & & & \\
\hline Simulium & 6 & $.45 \%$ & 57 & $1.82 \%$ \\
\hline Tipulidae (CRANE FLIES) & & & & \\
\hline Antocha & 159 & $11.98 \%$ & 150 & $4.78 \%$ \\
\hline
\end{tabular}


Table 5. Benthic-macroinvertebrate data-Continued

01480950 (Site 39) - East Branch Brandywine Creek at Wawaset, Pa.

\begin{tabular}{|c|c|c|c|c|c|c|}
\hline Date & \multicolumn{2}{|c|}{$11 / 20 / 95$} & \multicolumn{2}{|c|}{$10 / 15 / 96$} & \multicolumn{2}{|c|}{$10 / 29 / 97$} \\
\hline Total count & \multicolumn{2}{|c|}{307} & \multicolumn{2}{|c|}{880} & \multicolumn{2}{|c|}{2,123} \\
\hline Total number of taxa & \multicolumn{2}{|c|}{33} & \multicolumn{2}{|c|}{32} & \multicolumn{2}{|c|}{30} \\
\hline Organism & Count & Percent & Count & Percent & Count & Percent \\
\hline \multicolumn{7}{|l|}{ Platyhelminthes } \\
\hline \multicolumn{7}{|l|}{ Turbellaria (FLATWORMS) } \\
\hline \multicolumn{7}{|l|}{ Tricladida } \\
\hline Planariidae & 4 & $1.30 \%$ & 2 & $0.23 \%$ & 28 & $1.32 \%$ \\
\hline \multicolumn{7}{|l|}{ Nemertea (PROBOSCIS WORMS) } \\
\hline \multicolumn{7}{|l|}{ Enopla } \\
\hline \multicolumn{7}{|l|}{ Hoplonemertea } \\
\hline \multicolumn{7}{|l|}{ Tetrastemmatidae } \\
\hline Prostoma & 1 & $.33 \%$ & 2 & $.23 \%$ & - & \\
\hline \multicolumn{7}{|l|}{ Mollusca } \\
\hline Gastropoda (SNAILS) & & & & & & \\
\hline Basommatophora & & & & & & \\
\hline Ancylidae & & & & & & \\
\hline Ferrissia & 2 & $.65 \%$ & 4 & $.45 \%$ & - & \\
\hline Physidae & & & & & & \\
\hline Physa & 3 & $.98 \%$ & - & & - & \\
\hline Planorbidae & & & & & & \\
\hline Gyraulus & 1 & $.33 \%$ & - & & 1 & $.05 \%$ \\
\hline Bivalvia (CLAMS) & & & & & & \\
\hline Veneroida & & & & & & \\
\hline Sphaeriidae & 2 & $.65 \%$ & 1 & $.11 \%$ & - & \\
\hline Annelida (SEGMENTED WORMS) & & & & & & \\
\hline Oligochaeta & & & & & & \\
\hline Tubificida & & & & & & \\
\hline Naididae & 2 & $.65 \%$ & 51 & $5.80 \%$ & - & \\
\hline Tubificidae & 1 & $.33 \%$ & - & & - & \\
\hline Arthropoda & & & & & & \\
\hline Acariformes & & & & & & \\
\hline Hydrachnidia (WATER MITES) & 4 & $1.30 \%$ & 32 & $3.64 \%$ & 104 & $4.90 \%$ \\
\hline Crustacea & & & & & & \\
\hline Cladocera & - & & - & & 2 & $.09 \%$ \\
\hline Amphipoda (SCUDS) & & & & & & \\
\hline Gammaridae & & & & & & \\
\hline Gammarus & - & & 1 & $.11 \%$ & - & \\
\hline Insecta & & & & & & \\
\hline Ephemeroptera (MAYFLIES) & & & & & & \\
\hline Baetidae & & & & & & \\
\hline Baetis & - & & - & & 5 & $.24 \%$ \\
\hline Caenidae & & & & & & \\
\hline Caenis & 30 & $9.77 \%$ & 1 & $.11 \%$ & - & \\
\hline Ephemerellidae & & & & & & \\
\hline Ephemerella & 11 & $3.58 \%$ & 6 & $.68 \%$ & 167 & $7.87 \%$ \\
\hline Heptageniidae & & & & & & \\
\hline Stenonema & 8 & $2.61 \%$ & 5 & $.57 \%$ & 69 & $3.25 \%$ \\
\hline Isonychiidae & & & & & & \\
\hline Isonychia & 2 & $.65 \%$ & - & & 3 & $.14 \%$ \\
\hline Leptohyphidae & & & & & & \\
\hline Tricorythodes & 5 & $1.63 \%$ & - & & - & \\
\hline Potamanthidae & & & & & & \\
\hline Anthopotamus & 2 & $.65 \%$ & - & & - & \\
\hline Odonata (DRAGONFLIES AND DA & & & & & & \\
\hline Coenagrionidae & & & & & & \\
\hline Argia & 1 & $.33 \%$ & - & & 1 & $.05 \%$ \\
\hline
\end{tabular}


Table 5. Benthic-macroinvertebrate data-Continued

01480950 (Site 39) - East Branch Brandywine Creek at Wawaset, Pa.-Continued

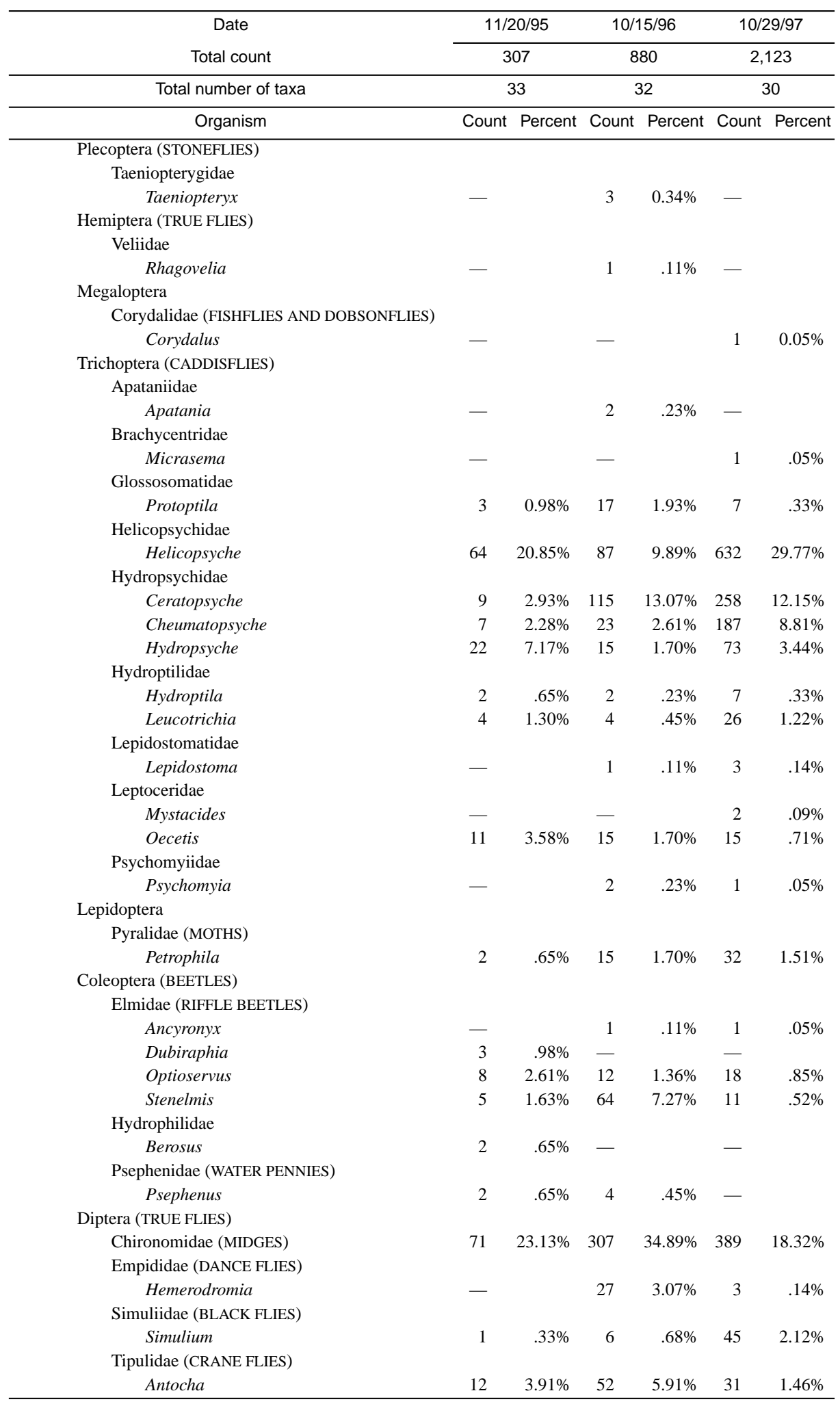


Table 5. Benthic-macroinvertebrate data-Continued

01481030 (Site 40) - Brandywine Creek near Chadds Ford, Pa.

\begin{tabular}{|c|c|c|c|c|}
\hline Date & \multicolumn{2}{|c|}{ 10/13/95 } & \multicolumn{2}{|c|}{$11 / 13 / 97$} \\
\hline Total count & \multicolumn{2}{|c|}{1,208} & \multicolumn{2}{|c|}{3,000} \\
\hline Total number of taxa & \multicolumn{2}{|c|}{33} & \multicolumn{2}{|c|}{41} \\
\hline Organism & Count & Percent & Count & Percent \\
\hline \multicolumn{5}{|l|}{ Platyhelminthes } \\
\hline \multicolumn{5}{|l|}{ Turbellaria (FLATWORMS) } \\
\hline \multicolumn{5}{|l|}{ Tricladida } \\
\hline Planariidae & 62 & $5.13 \%$ & 66 & $2.20 \%$ \\
\hline \multicolumn{5}{|l|}{ Nemertea (PROBOSCIS WORMS) } \\
\hline \multicolumn{5}{|l|}{ Enopla } \\
\hline \multicolumn{5}{|l|}{ Hoplonemertea } \\
\hline \multicolumn{5}{|l|}{ Tetrastemmatidae } \\
\hline Prostoma & 1 & $.08 \%$ & - & \\
\hline \multicolumn{5}{|l|}{ Mollusca } \\
\hline \multicolumn{5}{|l|}{ Gastropoda (SNAILS) } \\
\hline \multicolumn{5}{|l|}{ Basommatophora } \\
\hline \multicolumn{5}{|l|}{ Ancylidae } \\
\hline Ferrissia & 11 & $.91 \%$ & 11 & $.37 \%$ \\
\hline \multicolumn{5}{|l|}{ Physidae } \\
\hline Physa & 2 & $.17 \%$ & - & \\
\hline Planorbidae & & & & \\
\hline Gyraulus & - & & 1 & $.03 \%$ \\
\hline Mesogastropoda & & & & \\
\hline Hydrobiidae & & & & \\
\hline Amnicola & - & & 1 & $.03 \%$ \\
\hline Bivalvia (CLAMS) & & & & \\
\hline Veneroida & & & & \\
\hline Sphaeriidae & & & & \\
\hline Musculium & - & & 6 & $.20 \%$ \\
\hline Sphaerium & 10 & $.83 \%$ & - & \\
\hline Annelida (SEGMENTED WORMS) & & & & \\
\hline Oligochaeta & 3 & $.25 \%$ & - & \\
\hline Tubificida & & & & \\
\hline Naididae & 4 & $.33 \%$ & 2 & $.07 \%$ \\
\hline Arthropoda & & & & \\
\hline Acariformes & & & & \\
\hline Hydrachnidia (WATER MITES) & 37 & $3.06 \%$ & 94 & $3.13 \%$ \\
\hline Crustacea & & & & \\
\hline Amphipoda (SCUDS) & & & & \\
\hline Gammaridae & & & & \\
\hline Gammarus & - & & 6 & $.20 \%$ \\
\hline Podocopa (SEED SHRIMP) & - & & 2 & $.07 \%$ \\
\hline Insecta & & & & \\
\hline Ephemeroptera (MAYFLIES) & & & & \\
\hline Baetidae & & & & \\
\hline Baetis & 7 & $.58 \%$ & - & \\
\hline Pseudocloeon & 13 & $1.08 \%$ & 6 & $.20 \%$ \\
\hline Caenidae & & & & \\
\hline Caenis & 4 & $.33 \%$ & 2 & $.07 \%$ \\
\hline Ephemerellidae & & & & \\
\hline Ephemerella & 274 & $22.68 \%$ & 750 & $25.00 \%$ \\
\hline Heptageniidae & & & & \\
\hline Stenonema & 71 & $5.88 \%$ & 135 & $4.50 \%$ \\
\hline Isonychiidae & & & & \\
\hline Isonychia & 16 & $1.32 \%$ & 16 & $.53 \%$ \\
\hline Leptohyphidae & & & & \\
\hline Tricorythodes & - & & 27 & $.90 \%$ \\
\hline
\end{tabular}


Table 5. Benthic-macroinvertebrate data-Continued

01481030 (Site 40) - Brandywine Creek near Chadds Ford, Pa.-Continued

\begin{tabular}{|c|c|c|c|c|}
\hline Date & \multicolumn{2}{|c|}{$10 / 13 / 95$} & \multicolumn{2}{|c|}{ 11/13/97 } \\
\hline Total count & \multicolumn{2}{|c|}{1,208} & \multicolumn{2}{|c|}{3,000} \\
\hline Total number of taxa & \multicolumn{2}{|c|}{33} & \multicolumn{2}{|c|}{41} \\
\hline Organism & Count & Percent & Count & Percent \\
\hline \multicolumn{5}{|l|}{ Ephemeroptera (MAYFLIES) } \\
\hline \multicolumn{5}{|l|}{ Potamanthidae } \\
\hline Anthopotamus & - & & 1 & $0.03 \%$ \\
\hline \multicolumn{5}{|c|}{ Odonata (DRAGONFLIES AND DAMSELFLIES) } \\
\hline \multicolumn{5}{|c|}{ Coenagrionidae } \\
\hline Argia & 1 & $0.08 \%$ & 1 & $.03 \%$ \\
\hline \multicolumn{5}{|l|}{ Plecoptera (STONEFLIES) } \\
\hline Chloroperlidae & 5 & $.41 \%$ & - & \\
\hline \multicolumn{5}{|l|}{ Perlidae } \\
\hline Acroneuria & - & & 18 & $.60 \%$ \\
\hline Paragnetina & - & & 1 & $.03 \%$ \\
\hline \multicolumn{5}{|l|}{ Taeniopterygidae } \\
\hline Strophopteryx & - & & 39 & $1.30 \%$ \\
\hline Taeniopteryx & 2 & $.17 \%$ & 7 & $.23 \%$ \\
\hline \multicolumn{5}{|l|}{ Megaloptera } \\
\hline \multicolumn{5}{|c|}{ Corydalidae (FISHFLIES AND DOBSONFLIES) } \\
\hline Corydalus & - & & 2 & $.07 \%$ \\
\hline \multicolumn{5}{|l|}{ Trichoptera (CADDISFLIES) } \\
\hline \multicolumn{5}{|l|}{ Glossosomatidae } \\
\hline Protoptila & 11 & $.91 \%$ & 9 & $.30 \%$ \\
\hline \multicolumn{5}{|l|}{ Helicopsychidae } \\
\hline Helicopsyche & 6 & $.50 \%$ & 57 & $1.90 \%$ \\
\hline Hydropsychidae & & & & \\
\hline Ceratopsyche & 62 & $5.13 \%$ & 264 & $8.80 \%$ \\
\hline Cheumatopsyche & 153 & $12.67 \%$ & 240 & $8.00 \%$ \\
\hline Hydropsyche & 90 & $7.45 \%$ & 95 & $3.17 \%$ \\
\hline Hydroptilidae & & & & \\
\hline Hydroptila & - & & 10 & $.33 \%$ \\
\hline Leucotrichia & 84 & $6.95 \%$ & 17 & $.57 \%$ \\
\hline Lepidostomatidae & & & & \\
\hline Lepidostoma & 13 & $1.08 \%$ & 28 & $.93 \%$ \\
\hline Philopotamidae & & & & \\
\hline Chimarra & 1 & $.08 \%$ & 12 & $.40 \%$ \\
\hline Polycentropodidae & & & & \\
\hline Neureclipsis & - & & 3 & $.10 \%$ \\
\hline Psychomyiidae & & & & \\
\hline Psychomyia & - & & 1 & $.03 \%$ \\
\hline Lepidoptera & & & & \\
\hline Pyralidae (MOTHS) & & & & \\
\hline Petrophila & 65 & $5.38 \%$ & 10 & $.33 \%$ \\
\hline Coleoptera (BEETLES) & & & & \\
\hline Elmidae (RIFFLE BEETLES) & & & & \\
\hline Optioservus & 82 & $6.79 \%$ & 85 & $2.83 \%$ \\
\hline Oulimnius & 1 & $.08 \%$ & 8 & $.27 \%$ \\
\hline Stenelmis & 13 & $1.08 \%$ & 44 & $1.47 \%$ \\
\hline Diptera (TRUE FLIES) & & & & \\
\hline Chironomidae (MIDGES) & 79 & $6.54 \%$ & 836 & $27.87 \%$ \\
\hline Empididae (DANCE FLIES) & & & & \\
\hline Hemerodromia & 2 & $.17 \%$ & 6 & $.20 \%$ \\
\hline Simuliidae (BLACK FLIES) & & & & \\
\hline Simulium & 20 & $1.66 \%$ & 21 & $.70 \%$ \\
\hline Tipulidae (CRANE FLIES) & & & & \\
\hline Antocha & 3 & $.25 \%$ & 60 & $2.00 \%$ \\
\hline
\end{tabular}


Table 5. Benthic-macroinvertebrate data-Continued

01494900 (Site 31) - East Branch Big Elk Creek at Elkview, Pa.

\begin{tabular}{|c|c|c|c|c|c|c|}
\hline Date & \multicolumn{2}{|c|}{ 11/30/95 } & \multicolumn{2}{|c|}{ 10/25/96 } & \multicolumn{2}{|c|}{ 10/31/97 } \\
\hline Total count & \multicolumn{2}{|c|}{124} & \multicolumn{2}{|c|}{71} & \multicolumn{2}{|c|}{529} \\
\hline Total number of taxa & \multicolumn{2}{|c|}{14} & \multicolumn{2}{|c|}{11} & \multicolumn{2}{|c|}{13} \\
\hline Organism & Count & Percent & Count & Percent & Count & Percent \\
\hline \multicolumn{7}{|l|}{ Platyhelminthes } \\
\hline \multicolumn{7}{|l|}{ Turbellaria (FLATWORMS) } \\
\hline \multicolumn{7}{|l|}{ Tricladida } \\
\hline Planariidae & 3 & $2.42 \%$ & 1 & $1.41 \%$ & 1 & $0.19 \%$ \\
\hline \multicolumn{7}{|l|}{ Mollusca } \\
\hline \multicolumn{7}{|l|}{ Gastropoda (SNAILS) } \\
\hline \multicolumn{7}{|l|}{ Basommatophora } \\
\hline \multicolumn{7}{|l|}{ Ancylidae } \\
\hline Ferrissia & - & & 1 & $1.41 \%$ & 1 & $.19 \%$ \\
\hline \multicolumn{7}{|l|}{ Physidae } \\
\hline Physa & - & & 1 & $1.41 \%$ & - & \\
\hline \multicolumn{7}{|l|}{ Planorbidae } \\
\hline Gyraulus & - & & - & & 4 & $.76 \%$ \\
\hline Annelida (SEGMENTED WORMS) & & & & & & \\
\hline Oligochaeta & 1 & $.81 \%$ & - & & - & \\
\hline Tubificida & & & & & & \\
\hline Naididae & 40 & $32.26 \%$ & - & & 2 & $.38 \%$ \\
\hline Arthropoda & & & & & & \\
\hline Acariformes & & & & & & \\
\hline Hydrachnidia (WATER MITES) & 4 & $3.23 \%$ & 6 & $8.45 \%$ & 8 & $1.51 \%$ \\
\hline Crustacea & & & & & & \\
\hline Cyclopoida & 1 & $.81 \%$ & - & & - & \\
\hline Insecta & & & & & & \\
\hline Ephemeroptera (MAYFLIES) & & & & & & \\
\hline Ephemerellidae & & & & & & \\
\hline Ephemerella & 1 & $.81 \%$ & - & & - & \\
\hline Heptageniidae & & & & & & \\
\hline Stenonema & - & & - & & 1 & $.19 \%$ \\
\hline Isonychiidae & & & & & & \\
\hline Isonychia & 1 & $.81 \%$ & - & & - & \\
\hline Trichoptera (CADDISFLIES) & & & & & & \\
\hline Hydropsychidae & & & & & & \\
\hline Ceratopsyche & 2 & $1.61 \%$ & 5 & $7.04 \%$ & 5 & $.95 \%$ \\
\hline Cheumatopsyche & 12 & $9.68 \%$ & 8 & $11.27 \%$ & 6 & $1.13 \%$ \\
\hline Hydropsyche & - & & - & & 1 & $.19 \%$ \\
\hline Philopotamidae & & & & & & \\
\hline Chimarra & - & & 1 & $1.41 \%$ & - & \\
\hline Coleoptera (BEETLES) & & & & & & \\
\hline Elmidae (RIFFLE BEETLES) & & & & & & \\
\hline Ancyronyx & 1 & $.81 \%$ & - & & - & \\
\hline Microcylloepus & 1 & $.81 \%$ & - & & - & \\
\hline Optioservus & - & & 2 & $2.82 \%$ & - & \\
\hline Stenelmis & 4 & $3.23 \%$ & - & & - & \\
\hline Diptera (TRUE FLIES) & & & & & & \\
\hline Chironomidae (MIDGES) & 33 & $26.61 \%$ & 28 & $39.44 \%$ & 280 & $52.93 \%$ \\
\hline Empididae (DANCE FLIES) & & & & & & \\
\hline Hemerodromia & - & & - & & 2 & $.38 \%$ \\
\hline Simuliidae (BLACK FLIES) & & & & & & \\
\hline Simulium & - & & 2 & $2.82 \%$ & 1 & $.19 \%$ \\
\hline Tipulidae (CRANE FLIES) & & & & & & \\
\hline Antocha & 20 & $16.13 \%$ & 16 & $22.54 \%$ & 217 & $41.02 \%$ \\
\hline
\end{tabular}


Table 5. Benthic-macroinvertebrate data-Continued

01494950 (Site 32) - West Branch Big Elk Creek near Oxford, Pa.

\begin{tabular}{|c|c|c|c|c|}
\hline Date & \multicolumn{2}{|c|}{$11 / 30 / 95$} & \multicolumn{2}{|c|}{$10 / 25 / 96$} \\
\hline Total count & \multicolumn{2}{|c|}{85} & \multicolumn{2}{|c|}{290} \\
\hline Total number of taxa & \multicolumn{2}{|c|}{15} & \multicolumn{2}{|c|}{20} \\
\hline Organism & Count & Percent & Count & Percent \\
\hline \multicolumn{5}{|l|}{ Platyhelminthes } \\
\hline \multicolumn{5}{|l|}{ Turbellaria (FLATWORMS) } \\
\hline \multicolumn{5}{|l|}{ Tricladida } \\
\hline Planariidae & - & & 1 & $0.34 \%$ \\
\hline \multicolumn{5}{|l|}{ Mollusca } \\
\hline \multicolumn{5}{|l|}{ Gastropoda (SNAILS) } \\
\hline \multicolumn{5}{|l|}{ Basommatophora } \\
\hline \multicolumn{5}{|l|}{ Ancylidae } \\
\hline Ferrissia & 1 & $1.18 \%$ & - & \\
\hline \multicolumn{5}{|l|}{ Annelida (SEGMENTED WORMS) } \\
\hline \multicolumn{5}{|l|}{ Oligochaeta } \\
\hline Tubificida & & & & \\
\hline Naididae & 1 & $1.18 \%$ & - & \\
\hline Arthropoda & & & & \\
\hline Acariformes & & & & \\
\hline Hydrachnidia (WATER MITES) & - & & 19 & $6.55 \%$ \\
\hline Insecta & & & & \\
\hline Ephemeroptera (MAYFLIES) & & & & \\
\hline Baetidae & & & & \\
\hline Pseudocloeon & - & & 2 & $.69 \%$ \\
\hline Caenidae & & & & \\
\hline Caenis & 3 & $3.53 \%$ & - & \\
\hline Ephemerellidae & & & & \\
\hline Ephemerella & 3 & $3.53 \%$ & 6 & $2.07 \%$ \\
\hline Heptageniidae & & & & \\
\hline Stenacron & - & & 1 & $.34 \%$ \\
\hline Stenonema & 6 & $7.06 \%$ & 5 & $1.72 \%$ \\
\hline Isonychiidae & & & & \\
\hline Isonychia & 1 & $1.18 \%$ & - & \\
\hline Plecoptera (STONEFLIES) & & & & \\
\hline Capniidae & & & & \\
\hline Allocapnia & - & & 5 & $1.72 \%$ \\
\hline Trichoptera (CADDISFLIES) & & & & \\
\hline Brachycentridae & & & & \\
\hline Brachycentrus & 1 & $1.18 \%$ & - & \\
\hline Hydropsychidae & & & & \\
\hline Ceratopsyche & - & & 52 & $17.93 \%$ \\
\hline Cheumatopsyche & - & & 25 & $8.62 \%$ \\
\hline Hydropsyche & - & & 6 & $2.07 \%$ \\
\hline Hydroptilidae & & & & \\
\hline Leucotrichia & 1 & $1.18 \%$ & 1 & $.34 \%$ \\
\hline Philopotamidae & & & & \\
\hline Chimarra & - & & 1 & $.34 \%$ \\
\hline Uenoidae & & & & \\
\hline Neophylax & 1 & $1.18 \%$ & 1 & $.34 \%$ \\
\hline Coleoptera (BEETLES) & & & & \\
\hline Elmidae (RIFFLE BEETLES) & & & & \\
\hline Ancyronyx & 1 & $1.18 \%$ & - & \\
\hline Optioservus & 2 & $2.35 \%$ & 6 & $2.07 \%$ \\
\hline Oulimnius & - & & 2 & $.69 \%$ \\
\hline Stenelmis & 1 & $1.18 \%$ & 3 & $1.03 \%$ \\
\hline Diptera (TRUE FLIES) & & & & \\
\hline Chironomidae (MIDGES) & 16 & $18.82 \%$ & 83 & $28.62 \%$ \\
\hline Empididae(DANCE FLIES) & & & & \\
\hline Hemerodromia & 1 & $1.18 \%$ & 8 & $2.76 \%$ \\
\hline Simuliidae (BLACK FLIES) & & & & \\
\hline Simulium & - & & 9 & $3.10 \%$ \\
\hline Tipulidae (CRANE FLIES) & & & & \\
\hline Antocha & 46 & $54.12 \%$ & 54 & $18.62 \%$ \\
\hline
\end{tabular}


Table 5. Benthic-macroinvertebrate data-Continued

01578340 (Site 33) - East Branch Octoraro Creek at Christiana, Pa.

\begin{tabular}{|c|c|c|c|c|c|c|}
\hline Date & \multicolumn{2}{|c|}{$10 / 26 / 95$} & \multicolumn{2}{|c|}{$10 / 30 / 96$} & \multicolumn{2}{|c|}{$10 / 31 / 97$} \\
\hline Total count & \multicolumn{2}{|c|}{719} & \multicolumn{2}{|c|}{847} & \multicolumn{2}{|c|}{1,478} \\
\hline Total number of taxa & \multicolumn{2}{|c|}{26} & \multicolumn{2}{|c|}{25} & \multicolumn{2}{|c|}{29} \\
\hline Organism & Count & Percent & Count & Percent & Count & Percent \\
\hline \multicolumn{7}{|l|}{ Platyhelminthes } \\
\hline \multicolumn{7}{|l|}{ Turbellaria (FLATWORMS) } \\
\hline \multicolumn{7}{|l|}{ Tricladida } \\
\hline Planariidae & 44 & $6.12 \%$ & - & & 56 & $3.79 \%$ \\
\hline Nematoda (NEMATODES) & - & & - & & 3 & $.20 \%$ \\
\hline \multicolumn{7}{|l|}{ Nemertea (PROBOSCIS WORMS) } \\
\hline \multicolumn{7}{|l|}{ Enopla } \\
\hline \multicolumn{7}{|l|}{ Hoplonemertea } \\
\hline \multicolumn{7}{|l|}{ Tetrastemmatidae } \\
\hline Prostoma & 1 & $.14 \%$ & 33 & $3.90 \%$ & - & \\
\hline \multicolumn{7}{|l|}{ Mollusca } \\
\hline \multicolumn{7}{|l|}{ Gastropoda (SNAILS) } \\
\hline \multicolumn{7}{|l|}{ Basommatophora } \\
\hline \multicolumn{7}{|l|}{ Ancylidae } \\
\hline Ferrissia & - & & - & & 4 & $.27 \%$ \\
\hline Planorbidae & & & & & & \\
\hline Gyraulus & - & & 1 & $.12 \%$ & - & \\
\hline Bivalvia (CLAMS) & & & & & & \\
\hline Veneroida & & & & & & \\
\hline Sphaeriidae & - & & 2 & $.24 \%$ & 1 & $.07 \%$ \\
\hline Annelida (SEGMENTED WORMS) & & & & & & \\
\hline Oligochaeta & & & & & & \\
\hline Tubificida & & & & & & \\
\hline Naididae & 3 & $.42 \%$ & 30 & $3.54 \%$ & 1 & $.07 \%$ \\
\hline Arthropoda & & & & & & \\
\hline Acariformes & & & & & & \\
\hline Hydrachnidia (WATER MITES) & 15 & $2.09 \%$ & 10 & $1.18 \%$ & 16 & $1.08 \%$ \\
\hline Crustacea & & & & & & \\
\hline Amphipoda (SCUDS) & & & & & & \\
\hline Gammaridae & & & & & & \\
\hline Gammarus & - & & 1 & $.12 \%$ & 2 & $.14 \%$ \\
\hline Cyclopoida & - & & 1 & $.12 \%$ & - & \\
\hline Isopoda (SOW BUGS) & & & & & & \\
\hline Asellidae & & & & & & \\
\hline Caecidotea & 4 & $.56 \%$ & 5 & $.59 \%$ & - & \\
\hline Lirceus & - & & - & & 5 & $.34 \%$ \\
\hline Insecta & & & & & & \\
\hline Ephemeroptera (MAYFLIES) & & & & & & \\
\hline Baetidae & & & & & & \\
\hline Baetis & 1 & $.14 \%$ & - & & 1 & $.07 \%$ \\
\hline Ephemerellidae & & & & & & \\
\hline Ephemerella & 260 & $36.16 \%$ & 170 & $20.07 \%$ & 350 & $23.68 \%$ \\
\hline Heptageniidae & & & & & & \\
\hline Stenonema & 17 & $2.36 \%$ & 5 & $.59 \%$ & 23 & $1.56 \%$ \\
\hline Isonychiidae & & & & & & \\
\hline Isonychia & 1 & $.14 \%$ & - & & 1 & $.07 \%$ \\
\hline Leptohyphidae & & & & & & \\
\hline Tricorythodes & - & & - & & 1 & $.07 \%$ \\
\hline
\end{tabular}


Table 5. Benthic-macroinvertebrate data-Continued

01578340 (Site 33) - East Branch Octoraro Creek at Christiana, Pa.-Continued

\begin{tabular}{|c|c|c|c|c|c|c|}
\hline Date & \multicolumn{2}{|c|}{$10 / 26 / 95$} & \multicolumn{2}{|c|}{$10 / 30 / 96$} & \multicolumn{2}{|c|}{$10 / 31 / 97$} \\
\hline Total count & \multicolumn{2}{|c|}{719} & \multicolumn{2}{|c|}{847} & \multicolumn{2}{|c|}{1,478} \\
\hline Total number of taxa & \multicolumn{2}{|c|}{26} & \multicolumn{2}{|c|}{25} & \multicolumn{2}{|c|}{29} \\
\hline Organism & Count & Percent & Count & Percent & Count & Percent \\
\hline \multicolumn{7}{|l|}{ Plecoptera (STONEFLIES) } \\
\hline \multicolumn{7}{|l|}{ Perlidae } \\
\hline Agnetina & 1 & $0.14 \%$ & - & & - & \\
\hline \multicolumn{7}{|l|}{ Taeniopterygidae } \\
\hline Taeniopteryx & - & & - & & 1 & $0.07 \%$ \\
\hline \multicolumn{7}{|l|}{ Trichoptera (CADDISFLIES) } \\
\hline \multicolumn{7}{|l|}{ Brachycentridae } \\
\hline Micrasema & 2 & $.28 \%$ & - & & - & \\
\hline \multicolumn{7}{|l|}{ Hydropsychidae } \\
\hline Ceratopsyche & 44 & $6.12 \%$ & 58 & $6.85 \%$ & 20 & $1.35 \%$ \\
\hline Cheumatopsyche & 40 & $5.56 \%$ & 78 & $9.21 \%$ & 11 & $.74 \%$ \\
\hline Hydropsyche & 38 & $5.29 \%$ & 130 & $15.35 \%$ & 117 & $7.92 \%$ \\
\hline \multicolumn{7}{|l|}{ Hydroptilidae } \\
\hline Hydroptila & 9 & $1.25 \%$ & 1 & $.12 \%$ & 15 & $1.01 \%$ \\
\hline Leucotrichia & 12 & $1.67 \%$ & 32 & $3.78 \%$ & 220 & $14.88 \%$ \\
\hline \multicolumn{7}{|l|}{ Leptoceridae } \\
\hline Oecetis & 1 & $.14 \%$ & - & & 3 & $.20 \%$ \\
\hline \multicolumn{7}{|l|}{ Philopotamidae } \\
\hline Chimarra & 32 & $4.45 \%$ & 9 & $1.06 \%$ & 180 & $12.18 \%$ \\
\hline \multicolumn{7}{|l|}{ Psychomyiidae } \\
\hline Psychomyia & - & & - & & 1 & $.07 \%$ \\
\hline \multicolumn{7}{|l|}{ Lepidoptera (MOTHS) } \\
\hline \multicolumn{7}{|l|}{ Noctuidae } \\
\hline Archanara & - & & 1 & $.12 \%$ & - & \\
\hline \multicolumn{7}{|l|}{ Coleoptera (BEETLES) } \\
\hline \multicolumn{7}{|l|}{ Elmidae (RIFFLE BEETLES) } \\
\hline Ancyronyx & 1 & $.14 \%$ & - & & - & \\
\hline Dubiraphia & 1 & $.14 \%$ & - & & - & \\
\hline Macronychus & 1 & $.14 \%$ & - & & - & \\
\hline Optioservus & - & & 3 & $.35 \%$ & 5 & $.34 \%$ \\
\hline Stenelmis & 21 & $2.92 \%$ & 13 & $1.53 \%$ & 27 & $1.83 \%$ \\
\hline Psephenidae (WATER PENN & & & & & & \\
\hline Psephenus & 3 & $.42 \%$ & 7 & $.83 \%$ & 10 & $.68 \%$ \\
\hline Diptera (TRUE FLIES) & & & & & & \\
\hline Chironomidae (MIDGES) & 139 & $19.33 \%$ & 160 & $18.89 \%$ & 345 & $23.34 \%$ \\
\hline Empididae (DANCE FLIES) & & & & & & \\
\hline Hemerodromia & 1 & $.14 \%$ & 6 & $.71 \%$ & 1 & $.07 \%$ \\
\hline Simuliidae (BLACK FLIES) & & & & & & \\
\hline Simulium & - & & 65 & $7.67 \%$ & 6 & $.41 \%$ \\
\hline Tipulidae (CRANE FLIES) & & & & & & \\
\hline Antocha & 27 & $3.76 \%$ & 24 & $2.83 \%$ & 52 & $3.52 \%$ \\
\hline Dicranota & - & & 2 & $.24 \%$ & - & \\
\hline
\end{tabular}


Table 5. Benthic-macroinvertebrate data-Continued

01578343 (Site 34) - Valley Creek near Atglen, Pa.

\begin{tabular}{|c|c|c|c|c|}
\hline Date & \multicolumn{2}{|c|}{ 10/26/95 } & \multicolumn{2}{|c|}{ 10/30/96 } \\
\hline Total count & \multicolumn{2}{|c|}{460} & \multicolumn{2}{|c|}{1,855} \\
\hline Total number of taxa & \multicolumn{2}{|c|}{21} & \multicolumn{2}{|c|}{31} \\
\hline Organism & Count & Percent & Count & Percent \\
\hline Nematoda (NEMATODES) & - & & 2 & $.11 \%$ \\
\hline \multicolumn{5}{|l|}{ Nemertea (PROBOSCIS WORMS) } \\
\hline \multicolumn{5}{|l|}{ Enopla } \\
\hline \multicolumn{5}{|l|}{ Hoplonemertea } \\
\hline \multicolumn{5}{|l|}{ Tetrastemmatidae } \\
\hline Prostoma & - & & 7 & $0.38 \%$ \\
\hline \multicolumn{5}{|l|}{ Annelida (SEGMENTED WORMS) } \\
\hline \multicolumn{5}{|l|}{ Oligochaeta } \\
\hline \multicolumn{5}{|l|}{ Tubificida } \\
\hline Naididae & 18 & $3.91 \%$ & 153 & $8.25 \%$ \\
\hline \multicolumn{5}{|l|}{ Hirudinea (LEECHES) } \\
\hline \multicolumn{5}{|l|}{ Pharynobdellidae } \\
\hline Erpobdellidae & - & & 7 & $.38 \%$ \\
\hline \multicolumn{5}{|l|}{ Rhynehobdellida } \\
\hline \multicolumn{5}{|l|}{ Glossiphoniidae } \\
\hline Helobdella & 2 & $.43 \%$ & 4 & $.22 \%$ \\
\hline \multicolumn{5}{|l|}{ Arthropoda } \\
\hline \multicolumn{5}{|l|}{ Acariformes } \\
\hline Hydrachnidia (WATER MITES) & - & & 25 & $1.35 \%$ \\
\hline \multicolumn{5}{|l|}{ Crustacea } \\
\hline \multicolumn{5}{|l|}{ Amphipoda (SCUDS) } \\
\hline \multicolumn{5}{|l|}{ Gammaridae } \\
\hline Gammarus & 1 & $.22 \%$ & 5 & $.27 \%$ \\
\hline \multicolumn{5}{|l|}{ Isopoda (SOW BUGS) } \\
\hline Asellidae & & & & \\
\hline Caecidotea & - & & 46 & $2.48 \%$ \\
\hline Insecta & & & & \\
\hline Ephemeroptera (MAYFLIES) & & & & \\
\hline Baetidae & & & & \\
\hline Baetis & 1 & $.22 \%$ & 4 & $.22 \%$ \\
\hline Pseudocloeon & - & & 7 & $.38 \%$ \\
\hline Ephemerellidae & & & & \\
\hline Ephemerella & 3 & $.65 \%$ & 3 & $.16 \%$ \\
\hline Heptageniidae & & & & \\
\hline Stenonema & - & & 5 & $.27 \%$ \\
\hline Isonychiidae & & & & \\
\hline Isonychia & 1 & $.22 \%$ & 1 & $.05 \%$ \\
\hline Plecoptera (STONEFLIES) & & & & \\
\hline Capniidae & & & & \\
\hline Allocapnia & - & & 1 & $.05 \%$ \\
\hline Megaloptera & & & & \\
\hline Corydalidae (FISHFLIES AND DOBSONFLIES) & & & & \\
\hline Corydalus & 1 & $.22 \%$ & - & \\
\hline
\end{tabular}

Trichoptera (CADDISFLIES) 
Table 5. Benthic-macroinvertebrate data-Continued

01578343 (Site 34) - Valley Creek near Atglen, Pa.-Continued

\begin{tabular}{|c|c|c|c|c|}
\hline Date & \multicolumn{2}{|c|}{$10 / 26 / 95$} & \multicolumn{2}{|c|}{$10 / 30 / 96$} \\
\hline Total count & \multicolumn{2}{|c|}{460} & \multicolumn{2}{|c|}{1,855} \\
\hline Total number of taxa & \multicolumn{2}{|c|}{21} & \multicolumn{2}{|c|}{31} \\
\hline Organism & Count & Percent & Count & Percent \\
\hline \multicolumn{5}{|l|}{ Hydropsychidae } \\
\hline Ceratopsyche & 18 & $3.91 \%$ & 142 & $7.65 \%$ \\
\hline Cheumatopsyche & 71 & $15.43 \%$ & 114 & $6.15 \%$ \\
\hline Hydropsyche & 95 & $2.65 \%$ & 220 & $11.86 \%$ \\
\hline \multicolumn{5}{|l|}{ Hydroptilidae } \\
\hline Hydroptila & 1 & $.22 \%$ & 3 & $.16 \%$ \\
\hline Leucotrichia & 16 & $3.48 \%$ & 57 & $3.07 \%$ \\
\hline \multicolumn{5}{|l|}{ Leptoceridae } \\
\hline Ceraclea & 2 & $.43 \%$ & - & \\
\hline \multicolumn{5}{|l|}{ Philopotamidae } \\
\hline Dolophilodes & - & & 1 & $.05 \%$ \\
\hline \multicolumn{5}{|l|}{ Psychomyiidae } \\
\hline Psychomyia & 1 & $.22 \%$ & 13 & $.70 \%$ \\
\hline \multicolumn{5}{|l|}{ Coleoptera (BEETLES) } \\
\hline \multicolumn{5}{|l|}{ Elmidae (RIFFLE BEETLES) } \\
\hline Ancyronyx & 2 & $.43 \%$ & 4 & $.22 \%$ \\
\hline Optioservus & 3 & $.65 \%$ & 5 & $.27 \%$ \\
\hline Oulimnius & - & & 6 & $.32 \%$ \\
\hline Stenelmis & 38 & $8.26 \%$ & 35 & $1.89 \%$ \\
\hline \multicolumn{5}{|l|}{ Psephenidae } \\
\hline Psephenus & 5 & $1.09 \%$ & 12 & $.65 \%$ \\
\hline \multicolumn{5}{|l|}{ Diptera (TRUE FLIES) } \\
\hline Chironomidae (MIDGES) & 143 & $31.09 \%$ & 630 & $33.96 \%$ \\
\hline \multicolumn{5}{|l|}{ Empididae (DANCE FLIES) } \\
\hline Hemerodromia & - & & 36 & $1.94 \%$ \\
\hline \multicolumn{5}{|l|}{ Simuliidae (BLACK FLIES) } \\
\hline Simulium & 2 & $.43 \%$ & 49 & $2.64 \%$ \\
\hline \multicolumn{5}{|l|}{ Tipulidae (CRANE FLIES) } \\
\hline Antocha & 36 & $7.83 \%$ & 256 & $13.80 \%$ \\
\hline Dicranota & - & & 2 & $.11 \%$ \\
\hline
\end{tabular}


Table 6. Brillouin's diversity index, maximum diversity, minimum diversity, and relative evenness by site

01472080 (Site 10) - Pigeon Creek near Parker Ford, Pa.

\begin{tabular}{ccccccc}
\hline Year & $\begin{array}{c}\text { Total } \\
\text { organisms }\end{array}$ & $\begin{array}{c}\text { Total } \\
\text { taxa }\end{array}$ & $\begin{array}{c}\text { Brillouin's } \\
\text { diversity } \\
\text { index }(\mathrm{H})\end{array}$ & $\begin{array}{c}\text { Maximum } \\
\text { diversity } \\
\left(\mathrm{H}_{\text {max }}\right)\end{array}$ & $\begin{array}{c}\text { Minimum } \\
\text { diversity } \\
\left(\mathrm{H}_{\text {min }}\right)\end{array}$ & $\begin{array}{c}\text { Evenness } \\
(\mathrm{E})\end{array}$ \\
\hline 1995 & 930 & 39 & 3.28 & 5.30 & 0.40 & 0.59 \\
1996 & 1,685 & 40 & 1.97 & 5.25 & .25 & .34 \\
1997 & 1,918 & 36 & 3.43 & 5.23 & .21 & .64 \\
\hline
\end{tabular}

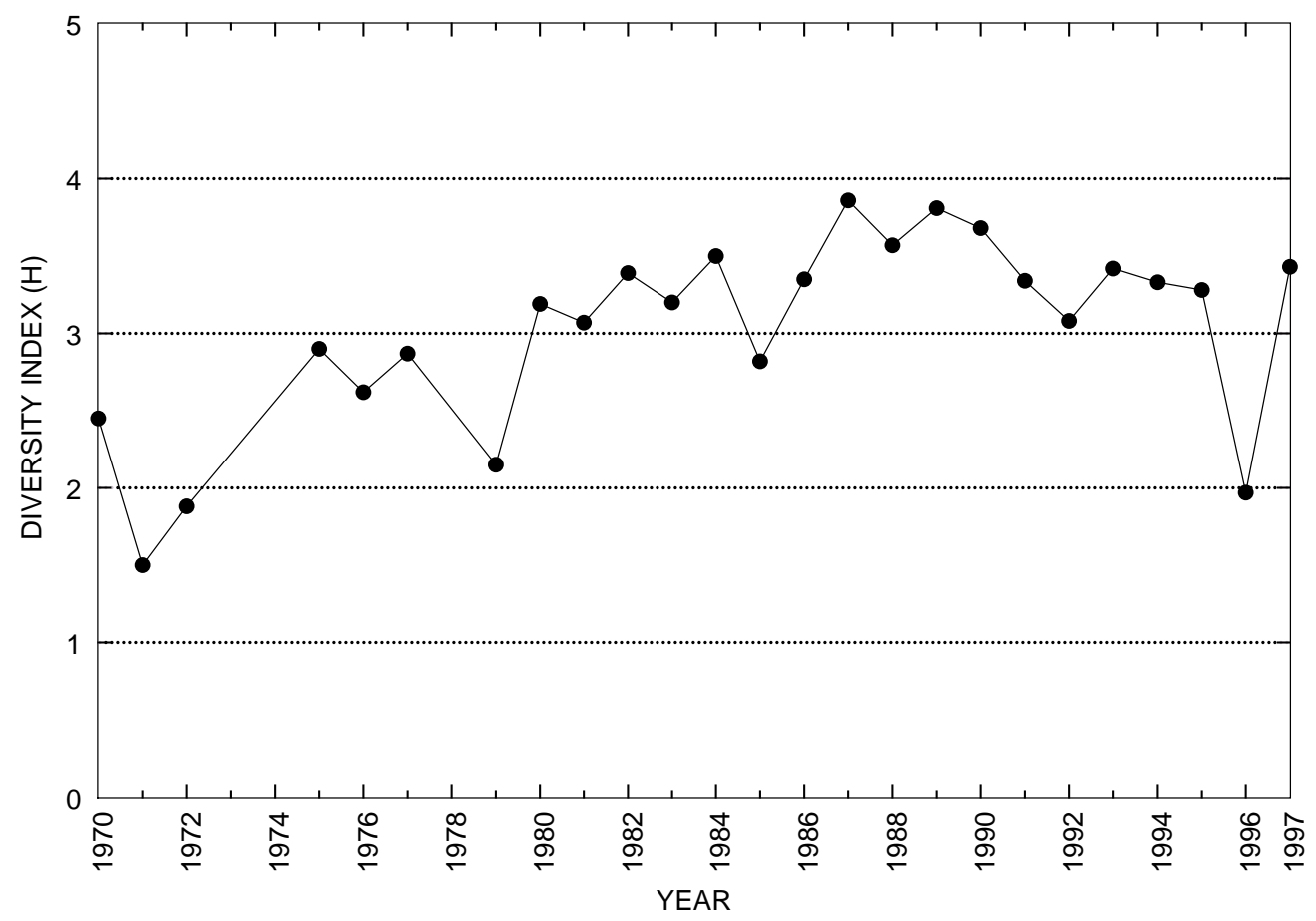


Table 6. Brillouin's diversity index, maximum diversity, minimum diversity, and relative evenness by site-Continued

[-, no data]

01472109 (Site 6) - Stony Run near Spring City, Pa.

\begin{tabular}{ccccccc}
\hline Year & $\begin{array}{c}\text { Total } \\
\text { organisms }\end{array}$ & $\begin{array}{c}\text { Total } \\
\text { taxa }\end{array}$ & $\begin{array}{c}\text { Brillouin's } \\
\text { diversity } \\
\text { index }(\mathrm{H})\end{array}$ & $\begin{array}{c}\text { Maximum } \\
\text { diversity } \\
\left(\mathrm{H}_{\text {max }}\right)\end{array}$ & $\begin{array}{c}\text { Minimum } \\
\text { diversity } \\
\left(\mathrm{H}_{\text {min }}\right)\end{array}$ & $\begin{array}{c}\text { Evenness } \\
(\mathrm{E})\end{array}$ \\
\hline 1995 & 744 & 39 & 3.97 & 5.13 & 0.49 & 0.75 \\
1996 & 1,860 & 34 & 3.58 & 5.09 & .19 & .69 \\
1997 & - & - & - & - & - & - \\
\hline
\end{tabular}

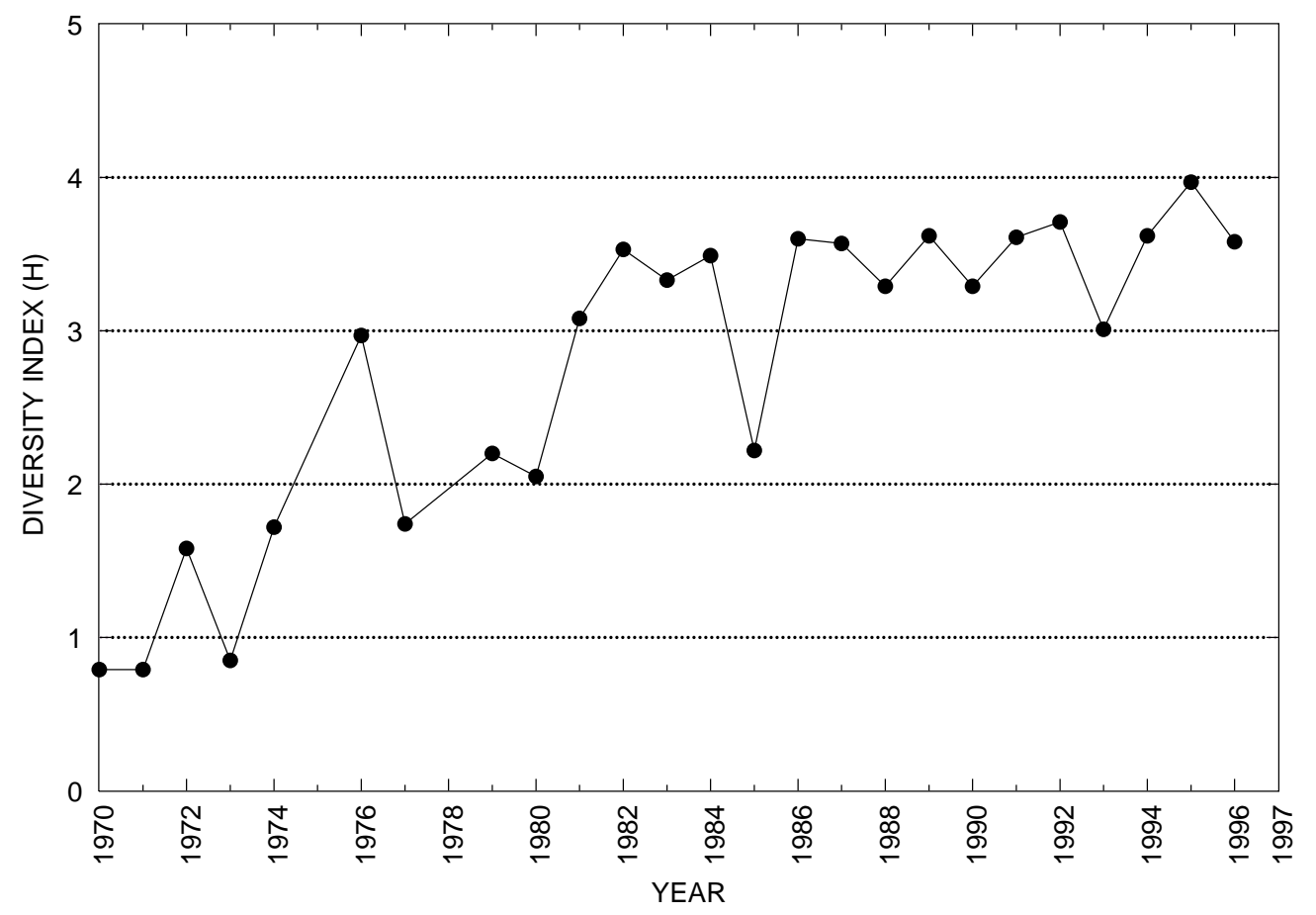


Table 6. Brillouin's diversity index, maximum diversity, minimum diversity, and relative evenness by site-Continued

[-, no data]

01472138 (Site 13) - French Creek near Coventryville, Pa.

\begin{tabular}{ccccccc}
\hline Year & $\begin{array}{c}\text { Total } \\
\text { organisms }\end{array}$ & $\begin{array}{c}\text { Total } \\
\text { taxa }\end{array}$ & $\begin{array}{c}\text { Brillouin's } \\
\text { diversity } \\
\text { index }(\mathrm{H})\end{array}$ & $\begin{array}{c}\text { Maximum } \\
\text { diversity } \\
\left(\mathrm{H}_{\text {max }}\right)\end{array}$ & $\begin{array}{c}\text { Minimum } \\
\text { diversity } \\
\left(\mathrm{H}_{\text {min }}\right)\end{array}$ & $\begin{array}{c}\text { Evenness } \\
(\mathrm{E})\end{array}$ \\
\hline 1995 & 414 & 34 & 3.79 & 4.90 & 0.69 & 0.74 \\
1996 & - & - & - & - & - & - \\
1997 & 1,080 & 32 & 3.76 & 4.88 & .35 & .64 \\
\hline
\end{tabular}

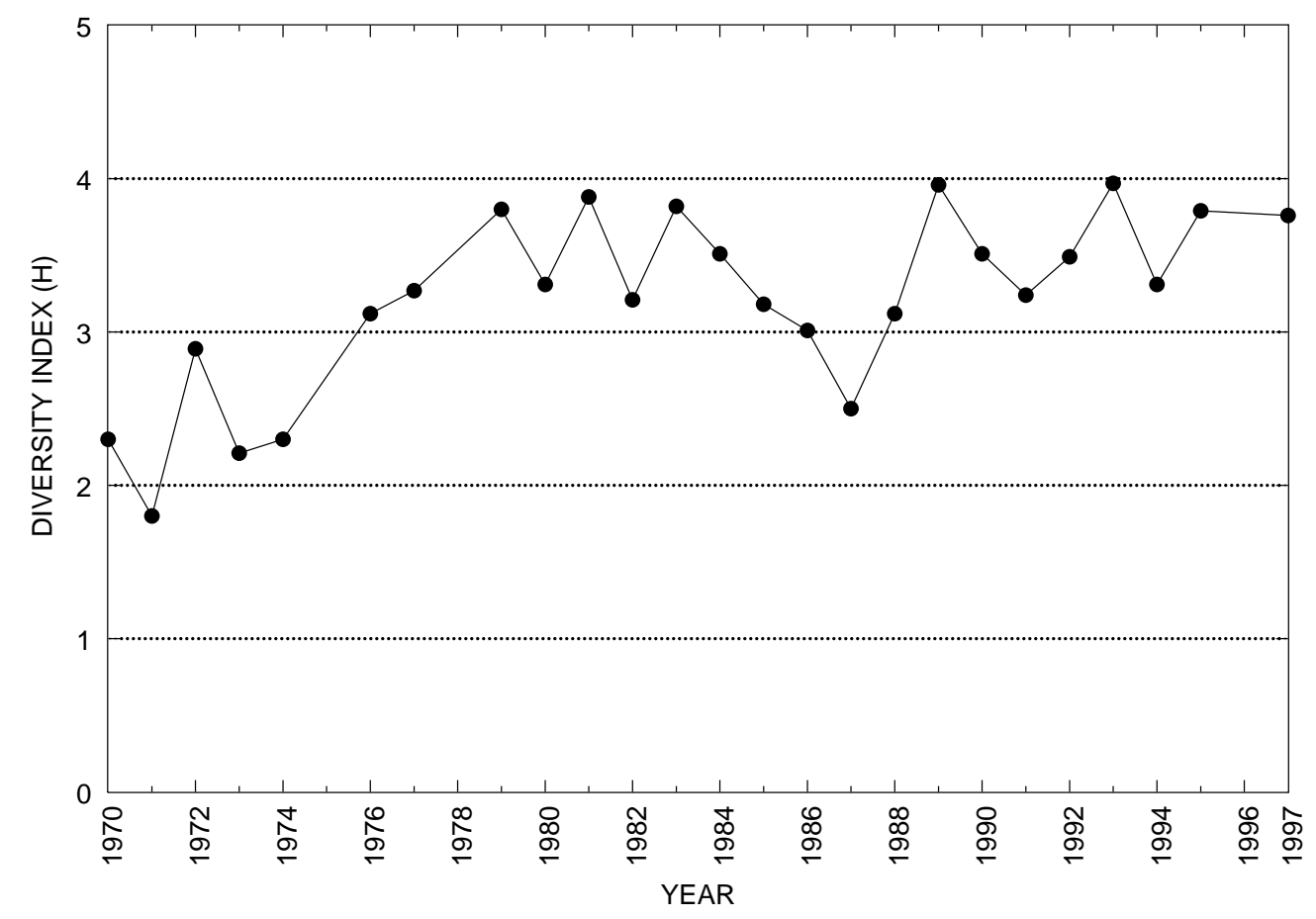


Table 6. Brillouin's diversity index, maximum diversity, minimum diversity, and relative evenness by site-Continued

$[-$, no data $]$

01472140 (Site 12) - South Branch French Creek at Coventryville, Pa.

\begin{tabular}{ccccccc}
\hline Year & $\begin{array}{c}\text { Total } \\
\text { organisms }\end{array}$ & $\begin{array}{c}\text { Total } \\
\text { taxa }\end{array}$ & $\begin{array}{c}\text { Brillouin's } \\
\text { diversity } \\
\text { index }(\mathrm{H})\end{array}$ & $\begin{array}{c}\text { Maximum } \\
\text { diversity } \\
\left(\mathrm{H}_{\text {max }}\right)\end{array}$ & $\begin{array}{c}\text { Minimum } \\
\text { diversity } \\
\left(\mathrm{H}_{\text {min }}\right)\end{array}$ & $\begin{array}{c}\text { Evenness } \\
(\mathrm{E})\end{array}$ \\
\hline 1995 & 585 & 32 & 3.83 & 4.89 & 0.49 & 0.76 \\
1996 & - & - & - & - & - & - \\
1997 & 1,527 & 32 & 3.23 & 5.00 & .21 & .63 \\
\hline
\end{tabular}

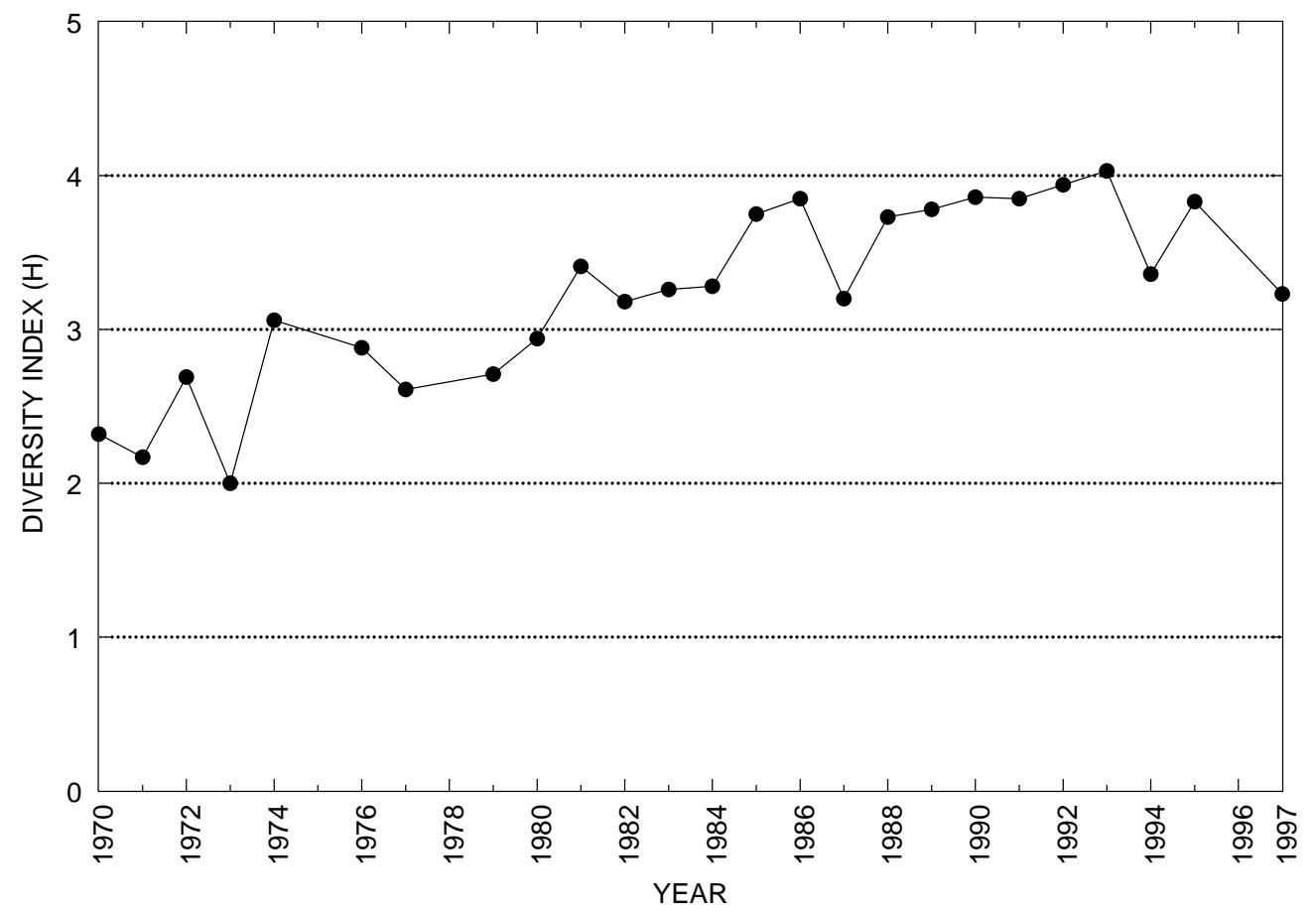


Table 6. Brillouin's diversity index, maximum diversity, minimum diversity, and relative evenness by site-Continued

$[-$, no data $]$

01472154 (Site 14) - French Creek near Pughtown, Pa.

\begin{tabular}{ccccccc}
\hline Year & $\begin{array}{c}\text { Total } \\
\text { organisms }\end{array}$ & $\begin{array}{c}\text { Total } \\
\text { taxa }\end{array}$ & $\begin{array}{c}\text { Brillouin's } \\
\text { diversity } \\
\text { index }(\mathrm{H})\end{array}$ & $\begin{array}{c}\text { Maximum } \\
\text { diversity } \\
\left(\mathrm{H}_{\text {max }}\right)\end{array}$ & $\begin{array}{c}\text { Minimum } \\
\text { diversity } \\
\left(\mathrm{H}_{\text {min }}\right)\end{array}$ & $\begin{array}{c}\text { Evenness } \\
(\mathrm{E})\end{array}$ \\
\hline 1995 & 388 & 34 & 3.80 & 4.96 & 0.73 & 0.73 \\
1996 & - & - & - & - & - & - \\
1997 & 800 & 33 & 3.41 & 4.95 & .38 & .66 \\
\hline
\end{tabular}

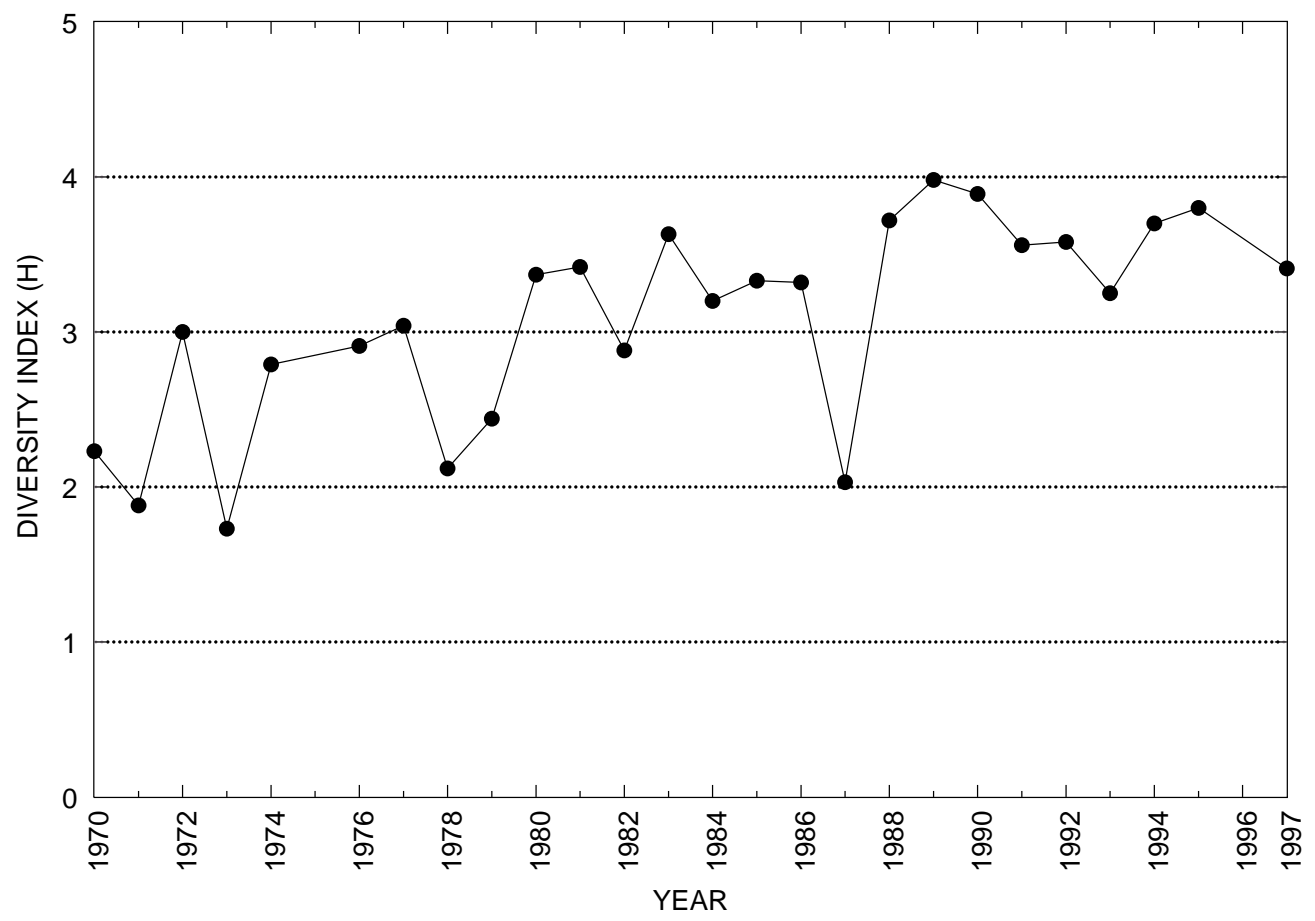


Table 6. Brillouin's diversity index, maximum diversity, minimum diversity, and relative evenness by site-Continued

01472157 (Site 15) - French Creek near Phoenixville, Pa.

\begin{tabular}{ccccccc}
\hline Year & $\begin{array}{c}\text { Total } \\
\text { organisms }\end{array}$ & $\begin{array}{c}\text { Total } \\
\text { taxa }\end{array}$ & $\begin{array}{c}\text { Brillouin's } \\
\text { diversity } \\
\text { index }(\mathrm{H})\end{array}$ & $\begin{array}{c}\text { Maximum } \\
\text { diversity } \\
\left(\mathrm{H}_{\max }\right)\end{array}$ & $\begin{array}{c}\text { Minimum } \\
\text { diversity } \\
\left(\mathrm{H}_{\min }\right)\end{array}$ & $\begin{array}{c}\text { Evenness } \\
(\mathrm{E})\end{array}$ \\
\hline 1995 & 281 & 35 & 3.60 & 5.14 & 1.00 & .76 \\
1996 & 198 & 21 & 2.86 & 4.25 & .23 & .60 \\
1997 & 1,677 & 37 & 3.80 & 5.16 & & .72 \\
\hline
\end{tabular}

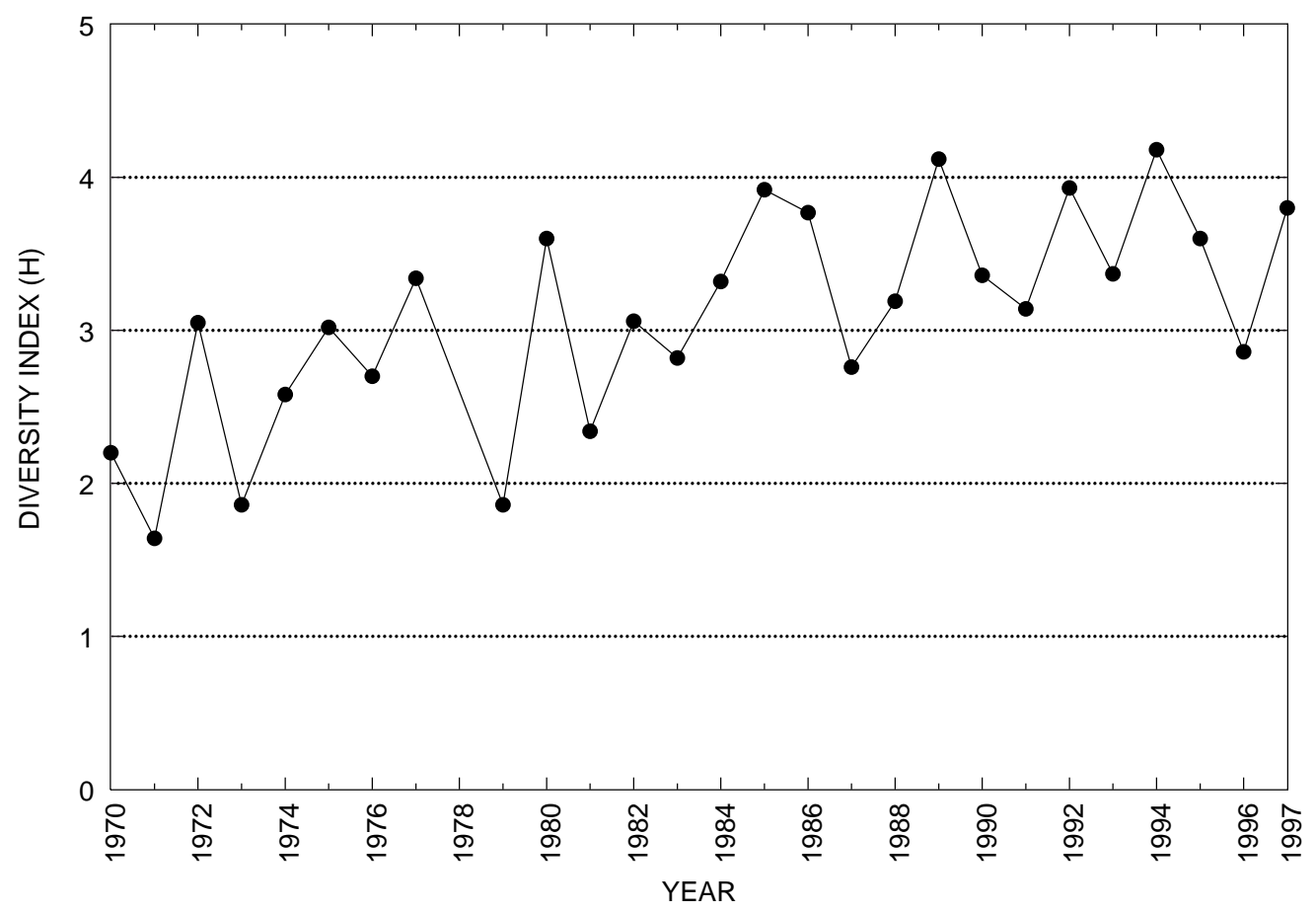


Table 6. Brillouin's diversity index, maximum diversity, minimum diversity, and relative evenness by site-Continued

$[-$, no data $]$

014721612 (Site 16) - French Creek at Railroad Bridge at Phoenixville, Pa.

\begin{tabular}{ccccccc}
\hline Year & $\begin{array}{c}\text { Total } \\
\text { organisms }\end{array}$ & $\begin{array}{c}\text { Total } \\
\text { taxa }\end{array}$ & $\begin{array}{c}\text { Brillouin's } \\
\text { diversity } \\
\text { index }(\mathrm{H})\end{array}$ & $\begin{array}{c}\text { Maximum } \\
\text { diversity } \\
\left(\mathrm{H}_{\text {max }}\right)\end{array}$ & $\begin{array}{c}\text { Minimum } \\
\text { diversity } \\
\left(\mathrm{H}_{\min }\right)\end{array}$ & $\begin{array}{c}\text { Evenness } \\
(\mathrm{E})\end{array}$ \\
\hline 1995 & 90 & 19 & 2.90 & 4.13 & 1.27 & 0.57 \\
1996 & - & - & - & - & - & - \\
1997 & 1,234 & 26 & 2.72 & 4.67 & .21 & .56 \\
\hline
\end{tabular}

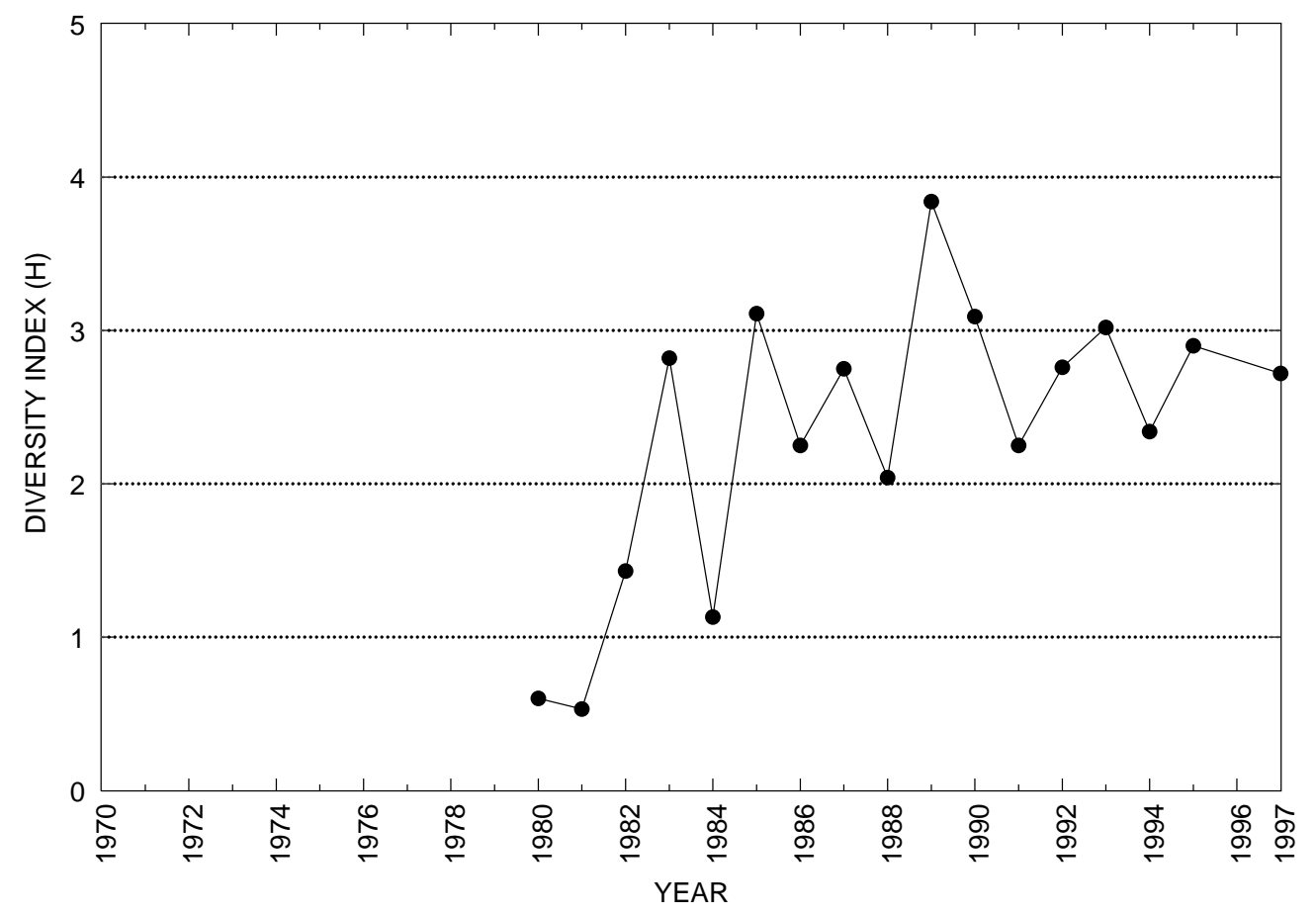


Table 6. Brillouin's diversity index, maximum diversity, minimum diversity, and relative evenness by site-Continued

$[-$, no data $]$

01472170 (Site 1) - Pickering Creek near Eagle, Pa.

\begin{tabular}{ccccccc}
\hline Year & $\begin{array}{c}\text { Total } \\
\text { organisms }\end{array}$ & $\begin{array}{c}\text { Total } \\
\text { taxa }\end{array}$ & $\begin{array}{c}\text { Brillouin's } \\
\text { diversity } \\
\text { index }(\mathrm{H})\end{array}$ & $\begin{array}{c}\text { Maximum } \\
\text { diversity } \\
\left(\mathrm{H}_{\text {max }}\right)\end{array}$ & $\begin{array}{c}\text { Minimum } \\
\text { diversity } \\
\left(\mathrm{H}_{\text {min }}\right)\end{array}$ & $\begin{array}{c}\text { Evenness } \\
(\mathrm{E})\end{array}$ \\
\hline 1995 & 533 & 23 & 2.88 & 4.41 & 0.37 & 0.62 \\
1996 & 757 & 28 & 3.44 & 4.68 & .34 & .71 \\
1997 & - & - & - & - & - & - \\
\hline
\end{tabular}

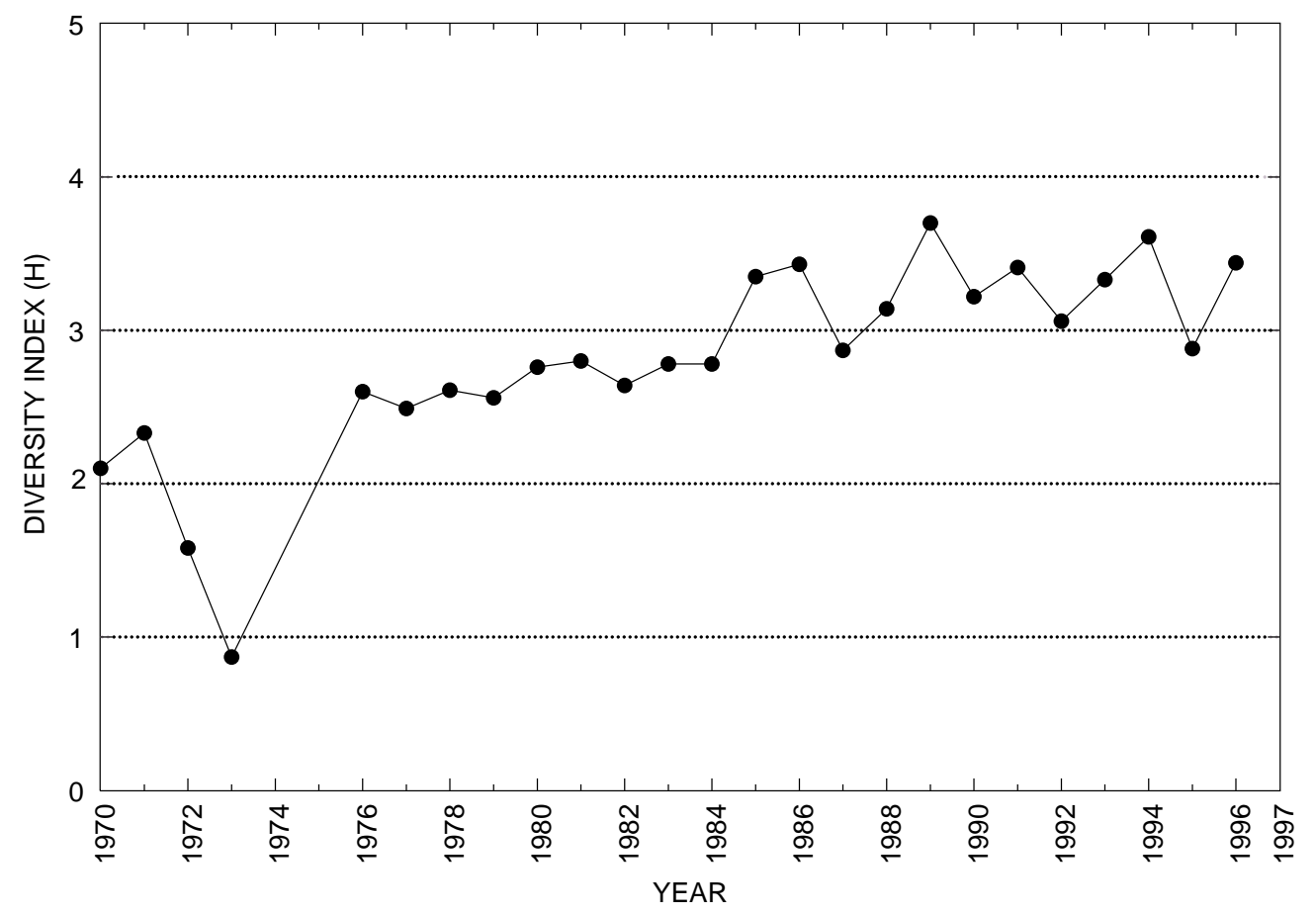


Table 6. Brillouin's diversity index, maximum diversity, minimum diversity, and relative evenness by site-Continued

$[-$, no data $]$

01472174 (Site 2) - Pickering Creek near Chester Springs, Pa.

\begin{tabular}{ccccccc}
\hline Year & $\begin{array}{c}\text { Total } \\
\text { organisms }\end{array}$ & $\begin{array}{c}\text { Total } \\
\text { taxa }\end{array}$ & $\begin{array}{c}\text { Brillouin's } \\
\text { diversity } \\
\text { index }(\mathrm{H})\end{array}$ & $\begin{array}{c}\text { Maximum } \\
\text { diversity } \\
\left(\mathrm{H}_{\text {max }}\right)\end{array}$ & $\begin{array}{c}\text { Minimum } \\
\text { diversity } \\
\left(\mathrm{H}_{\text {min }}\right)\end{array}$ & $\begin{array}{c}\text { Evenness } \\
(\mathrm{E})\end{array}$ \\
\hline 1995 & 223 & 26 & 3.51 & 4.60 & 0.87 & 0.71 \\
1996 & - & - & - & - & - & - \\
1997 & - & - & - & - & - & - \\
\hline
\end{tabular}

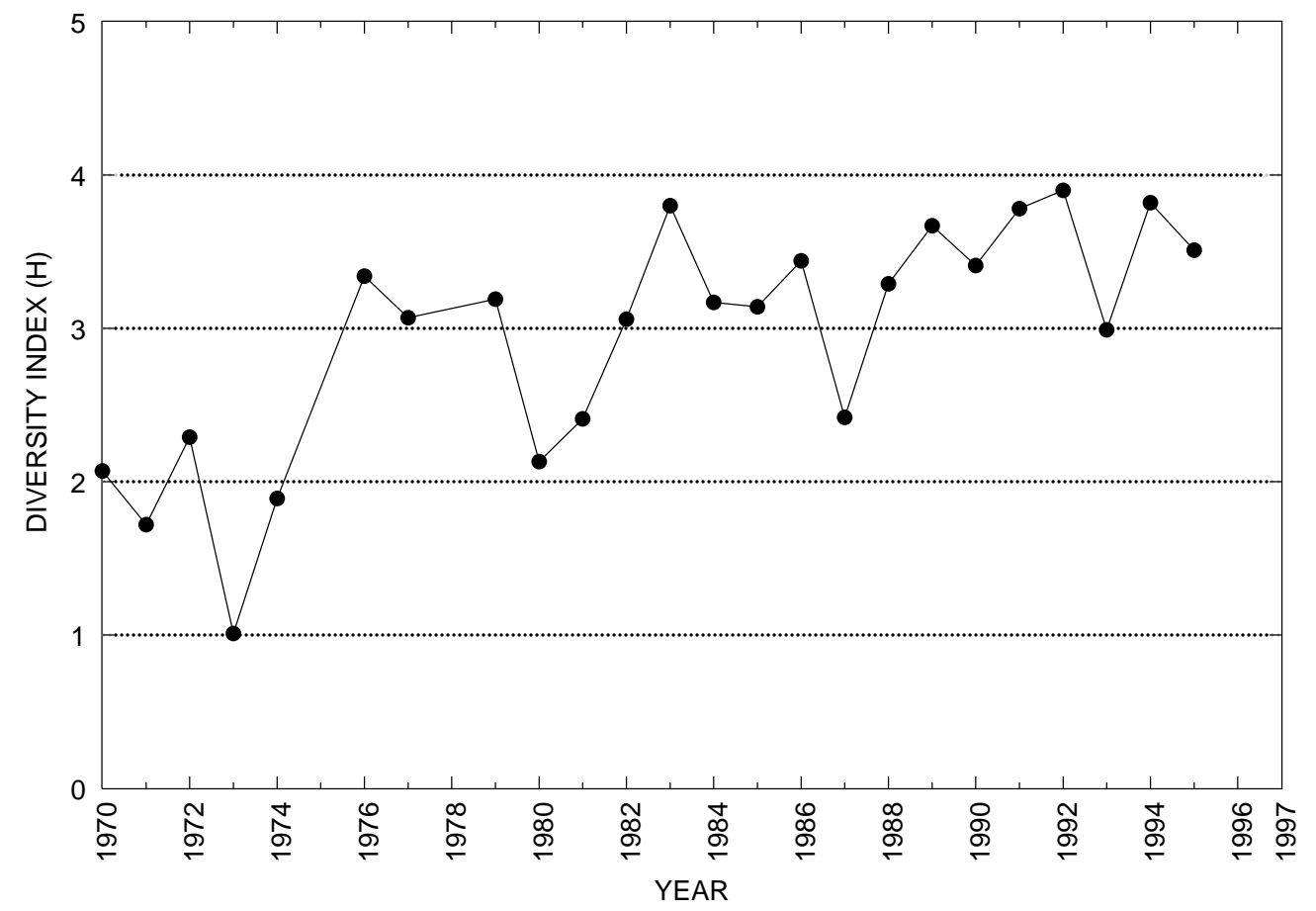


Table 6. Brillouin's diversity index, maximum diversity, minimum diversity, and relative evenness by site-Continued

$[-$, no data $]$

014721854 (Site 3) - Pickering Creek at Merlin, Pa.

\begin{tabular}{|c|c|c|c|c|c|c|}
\hline Year & $\begin{array}{c}\text { Total } \\
\text { organisms }\end{array}$ & $\begin{array}{l}\text { Total } \\
\text { taxa }\end{array}$ & $\begin{array}{l}\text { Brillouin's } \\
\text { diversity } \\
\text { index }(\mathrm{H})\end{array}$ & $\begin{array}{c}\text { Maximum } \\
\text { diversity } \\
\left(\mathrm{H}_{\max }\right)\end{array}$ & $\begin{array}{c}\text { Minimum } \\
\text { diversity } \\
\left(\mathrm{H}_{\min }\right)\end{array}$ & $\begin{array}{c}\text { Evenness } \\
(\mathrm{E})\end{array}$ \\
\hline 1995 & 457 & 30 & 3.64 & 4.94 & 0.58 & 0.70 \\
\hline 1996 & - & - & - & - & - & - \\
\hline 1997 & 1,463 & 41 & 3.24 & 5.35 & .29 & .58 \\
\hline
\end{tabular}

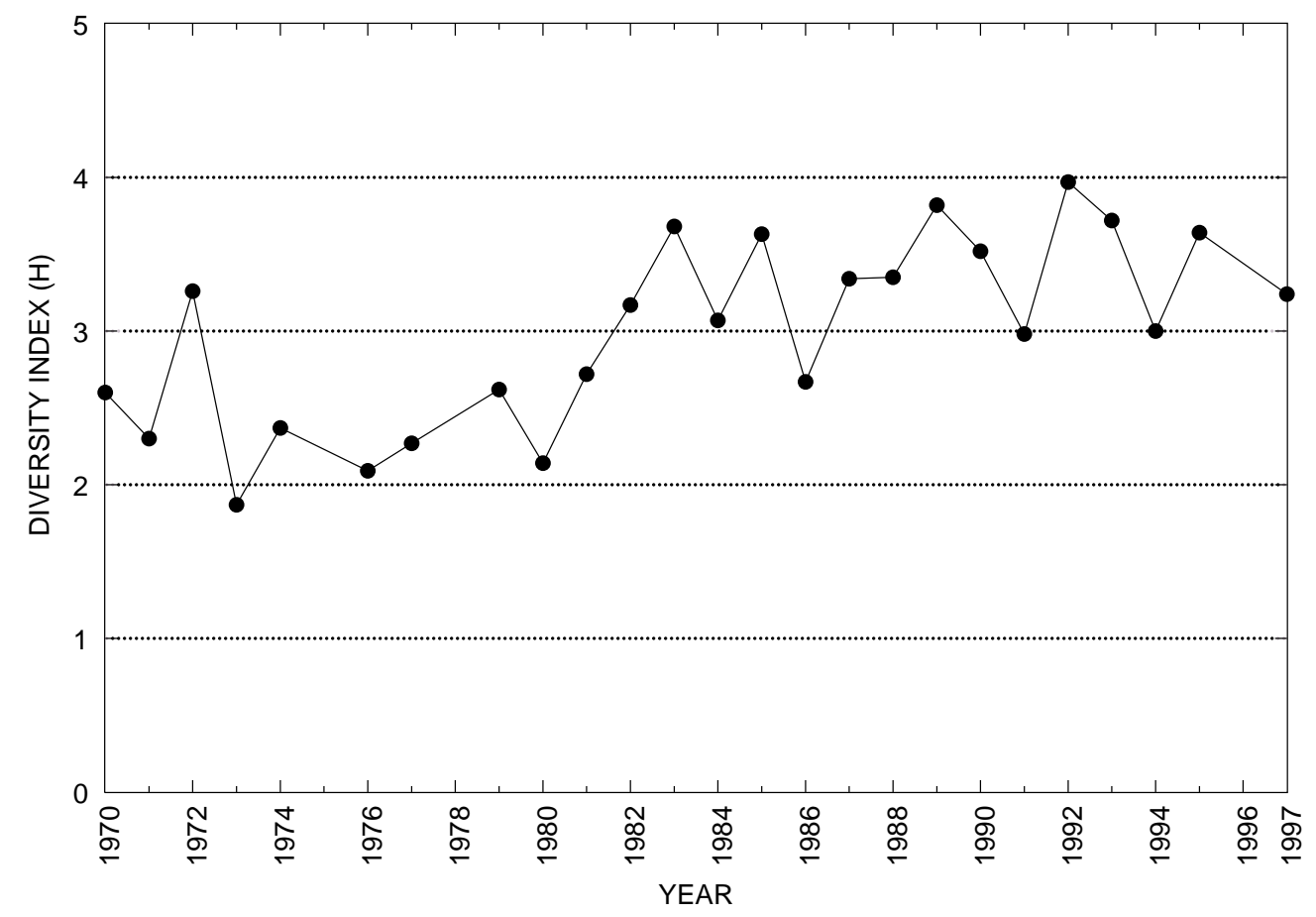


Table 6. Brillouin's diversity index, maximum diversity, minimum diversity, and relative evenness by site-Continued

$[-$, no data $]$

014721884 (Site 4) - Pickering Creek at Charlestown Road at Charlestown, Pa.

\begin{tabular}{ccccccc}
\hline Year & $\begin{array}{c}\text { Total } \\
\text { organisms }\end{array}$ & $\begin{array}{c}\text { Total } \\
\text { taxa }\end{array}$ & $\begin{array}{c}\text { Brillouin's } \\
\text { diversity } \\
\text { index }(\mathrm{H})\end{array}$ & $\begin{array}{c}\text { Maximum } \\
\text { diversity } \\
\left(\mathrm{H}_{\text {max }}\right)\end{array}$ & $\begin{array}{c}\text { Minimum } \\
\text { diversity } \\
\left(\mathrm{H}_{\text {min }}\right)\end{array}$ & $\begin{array}{c}\text { Evenness } \\
(\mathrm{E})\end{array}$ \\
\hline 1995 & 811 & 42 & 3.84 & 5.29 & 0.49 & 0.70 \\
1996 & 1,340 & 37 & 3.57 & 5.14 & .28 & .68 \\
1997 & - & - & - & - & - & - \\
\hline
\end{tabular}

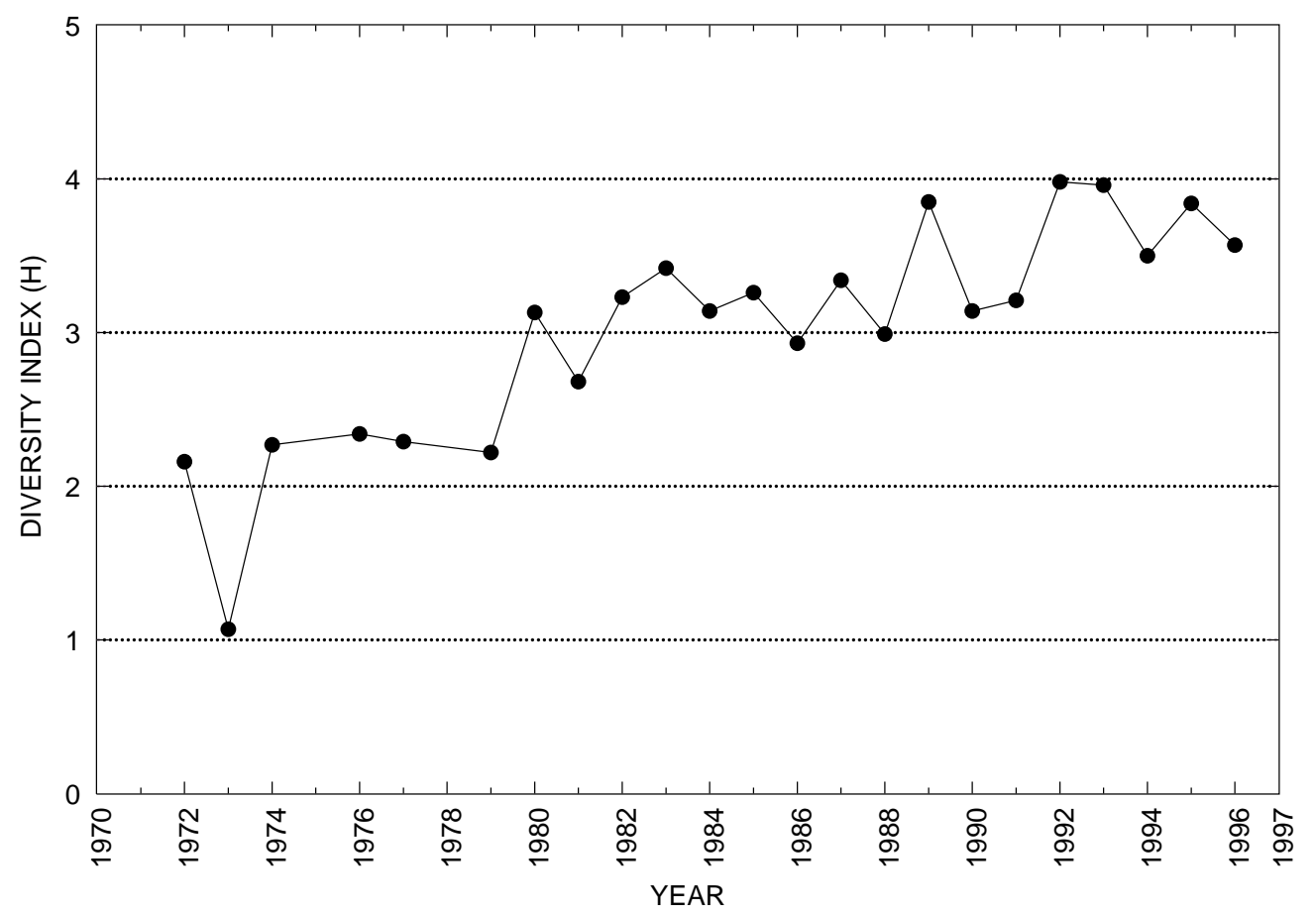


Table 6. Brillouin's diversity index, maximum diversity, minimum diversity, and relative evenness by site-Continued

01472190 (Site 5) - Pickering Creek near Phoenixville, Pa.

\begin{tabular}{ccccccc}
\hline Year & $\begin{array}{c}\text { Total } \\
\text { organisms }\end{array}$ & $\begin{array}{c}\text { Total } \\
\text { taxa }\end{array}$ & $\begin{array}{c}\text { Brillouin's } \\
\text { diversity } \\
\text { index }(\mathrm{H})\end{array}$ & $\begin{array}{c}\text { Maximum } \\
\text { diversity } \\
\left(\mathrm{H}_{\max }\right)\end{array}$ & $\begin{array}{c}\text { Minimum } \\
\text { diversity } \\
\left(\mathrm{H}_{\min }\right)\end{array}$ & $\begin{array}{c}\text { Evenness } \\
(\mathrm{E})\end{array}$ \\
\hline 1995 & 872 & 35 & 3.22 & 5.16 & 0.38 & .52 \\
1996 & 418 & 26 & 2.97 & 4.53 & .24 & .61 \\
1997 & 1,530 & 36 & 3.65 & 5.14 & & .70 \\
\hline
\end{tabular}

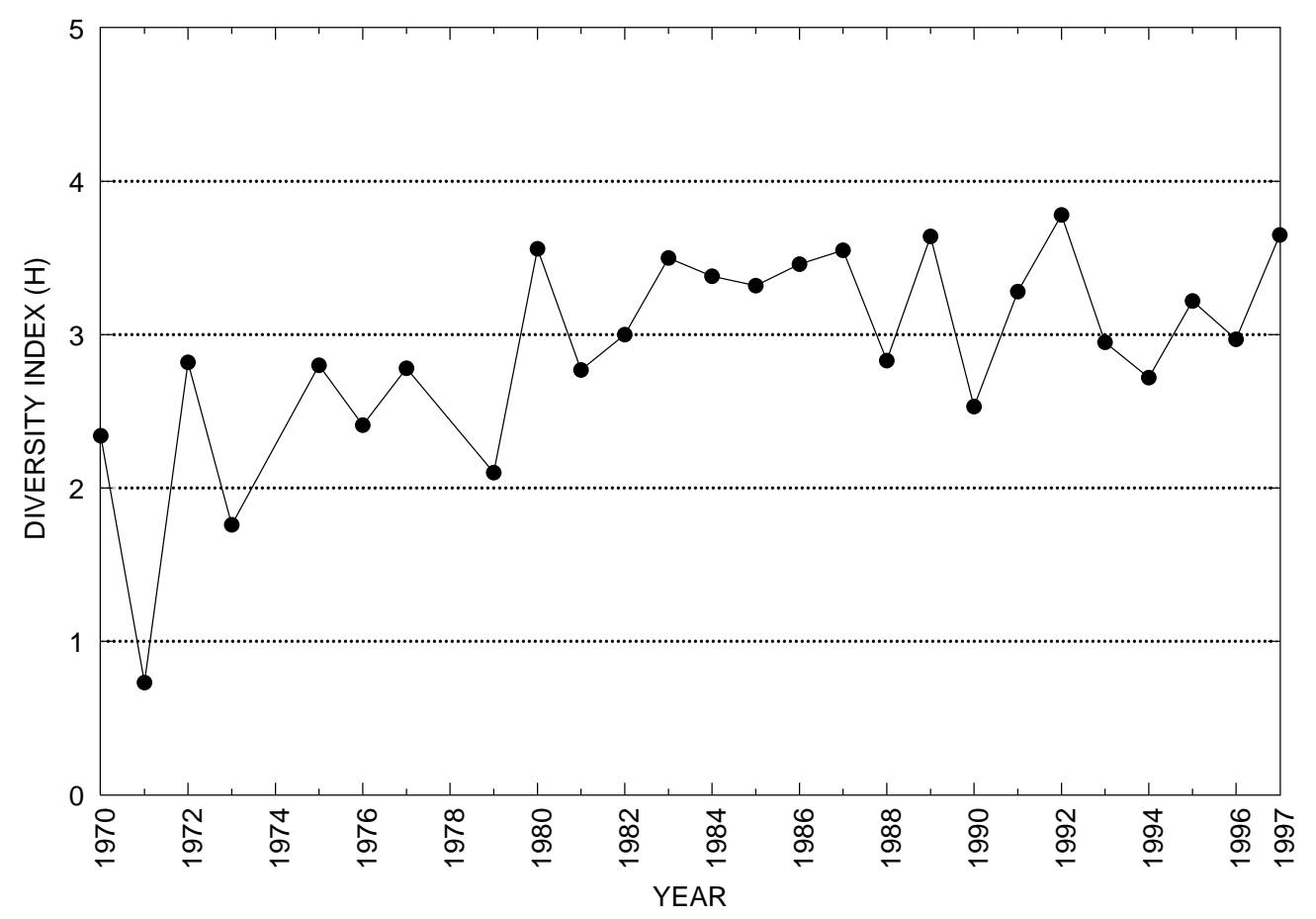


Table 6. Brillouin's diversity index, maximum diversity, minimum diversity, and relative evenness by site-Continued

$[-$, no data $]$

01473167 (Site 49) - Little Valley Creek at Howellville, Pa.

\begin{tabular}{ccccccc}
\hline Year & $\begin{array}{c}\text { Total } \\
\text { organisms }\end{array}$ & $\begin{array}{c}\text { Total } \\
\text { taxa }\end{array}$ & $\begin{array}{c}\text { Brillouin's } \\
\text { diversity } \\
\text { index }(\mathrm{H})\end{array}$ & $\begin{array}{c}\text { Maximum } \\
\text { diversity } \\
\left(\mathrm{H}_{\text {max }}\right)\end{array}$ & $\begin{array}{c}\text { Minimum } \\
\text { diversity } \\
\left(\mathrm{H}_{\text {min }}\right)\end{array}$ & $\begin{array}{c}\text { Evenness } \\
(\mathrm{E})\end{array}$ \\
\hline 1995 & 395 & 22 & 3.17 & 4.51 & 0.46 & 0.67 \\
1996 & 113 & 16 & 2.98 & 3.68 & .89 & .75 \\
1997 & - & - & - & - & - & - \\
\hline
\end{tabular}

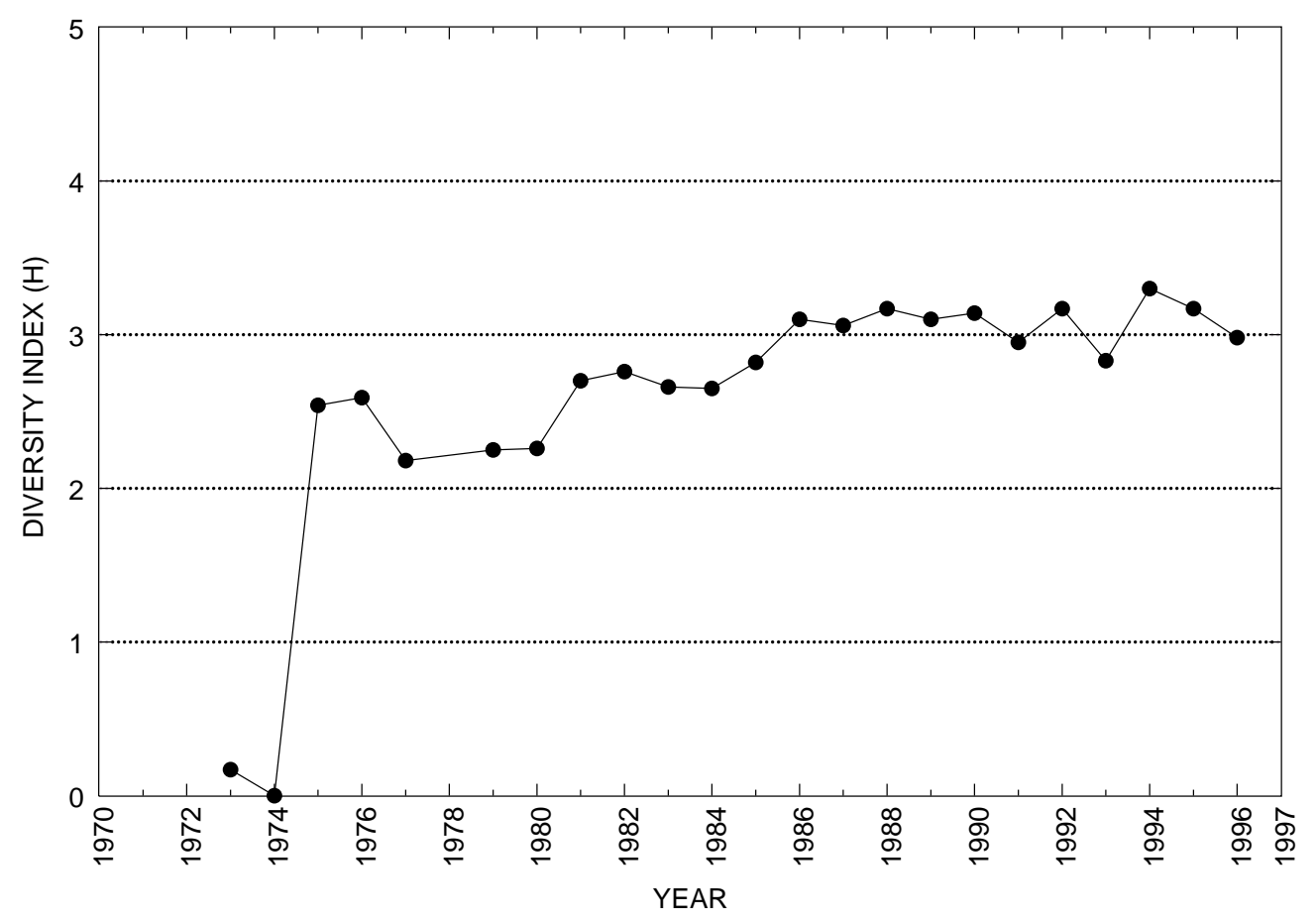


Table 6. Brillouin's diversity index, maximum diversity, minimum diversity, and relative evenness by site-Continued

$[-$, no data $]$

01473168 (Site 50) - Valley Creek near Valley Forge, Pa.

\begin{tabular}{ccccccc}
\hline Year & $\begin{array}{c}\text { Total } \\
\text { organisms }\end{array}$ & $\begin{array}{c}\text { Total } \\
\text { taxa }\end{array}$ & $\begin{array}{c}\text { Brillouin's } \\
\text { diversity } \\
\text { index }(\mathrm{H})\end{array}$ & $\begin{array}{c}\text { Maximum } \\
\text { diversity } \\
\left(\mathrm{H}_{\text {max }}\right)\end{array}$ & $\begin{array}{c}\text { Minimum } \\
\text { diversity } \\
\left(\mathrm{H}_{\text {min }}\right)\end{array}$ & $\begin{array}{c}\text { Evenness } \\
(\mathrm{E})\end{array}$ \\
\hline 1995 & 1,032 & 18 & 2.93 & 4.14 & 0.16 & 0.70 \\
1996 & 870 & 23 & 2.91 & 4.54 & .25 & .62 \\
1997 & - & - & - & - & - & - \\
\hline
\end{tabular}

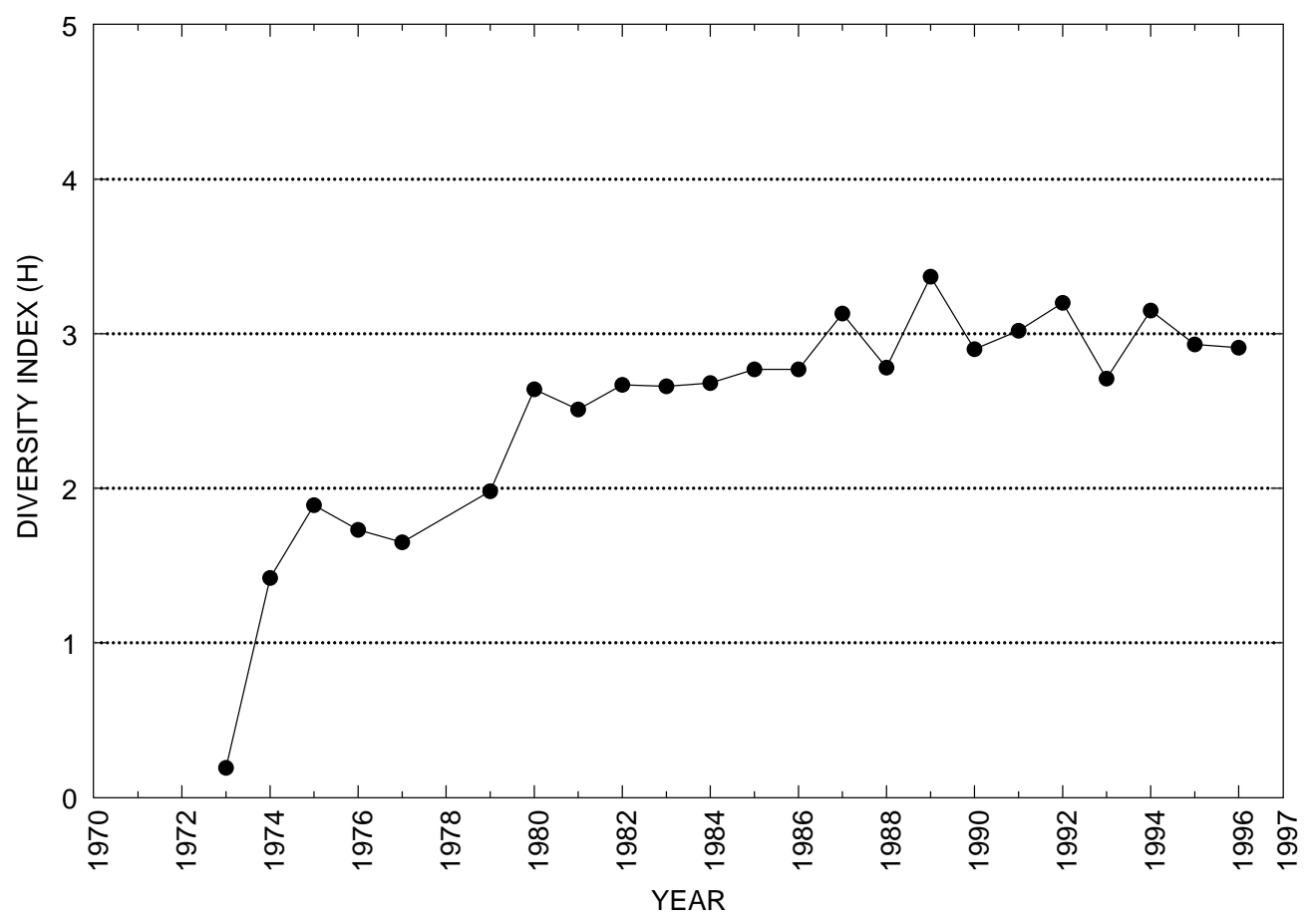


Table 6. Brillouin's diversity index, maximum diversity, minimum diversity, and relative evenness by site-Continued

$[-$, no data $]$

01475300 (Site 17) - Darby Creek at Waterloo Mills near Devon, Pa.

\begin{tabular}{ccccccc}
\hline Year & $\begin{array}{c}\text { Total } \\
\text { organisms }\end{array}$ & $\begin{array}{c}\text { Total } \\
\text { taxa }\end{array}$ & $\begin{array}{c}\text { Brillouin's } \\
\text { diversity } \\
\text { index }(\mathrm{H})\end{array}$ & $\begin{array}{c}\text { Maximum } \\
\text { diversity } \\
\left(\mathrm{H}_{\text {max }}\right)\end{array}$ & $\begin{array}{c}\text { Minimum } \\
\text { diversity } \\
\left(\mathrm{H}_{\text {min }}\right)\end{array}$ & $\begin{array}{c}\text { Evenness } \\
(\mathrm{E})\end{array}$ \\
\hline 1995 & 463 & 25 & 1.90 & 4.59 & 0.46 & 0.35 \\
1996 & 256 & 20 & 3.00 & 4.33 & .59 & .65 \\
1997 & - & - & - & - & - & - \\
\hline
\end{tabular}

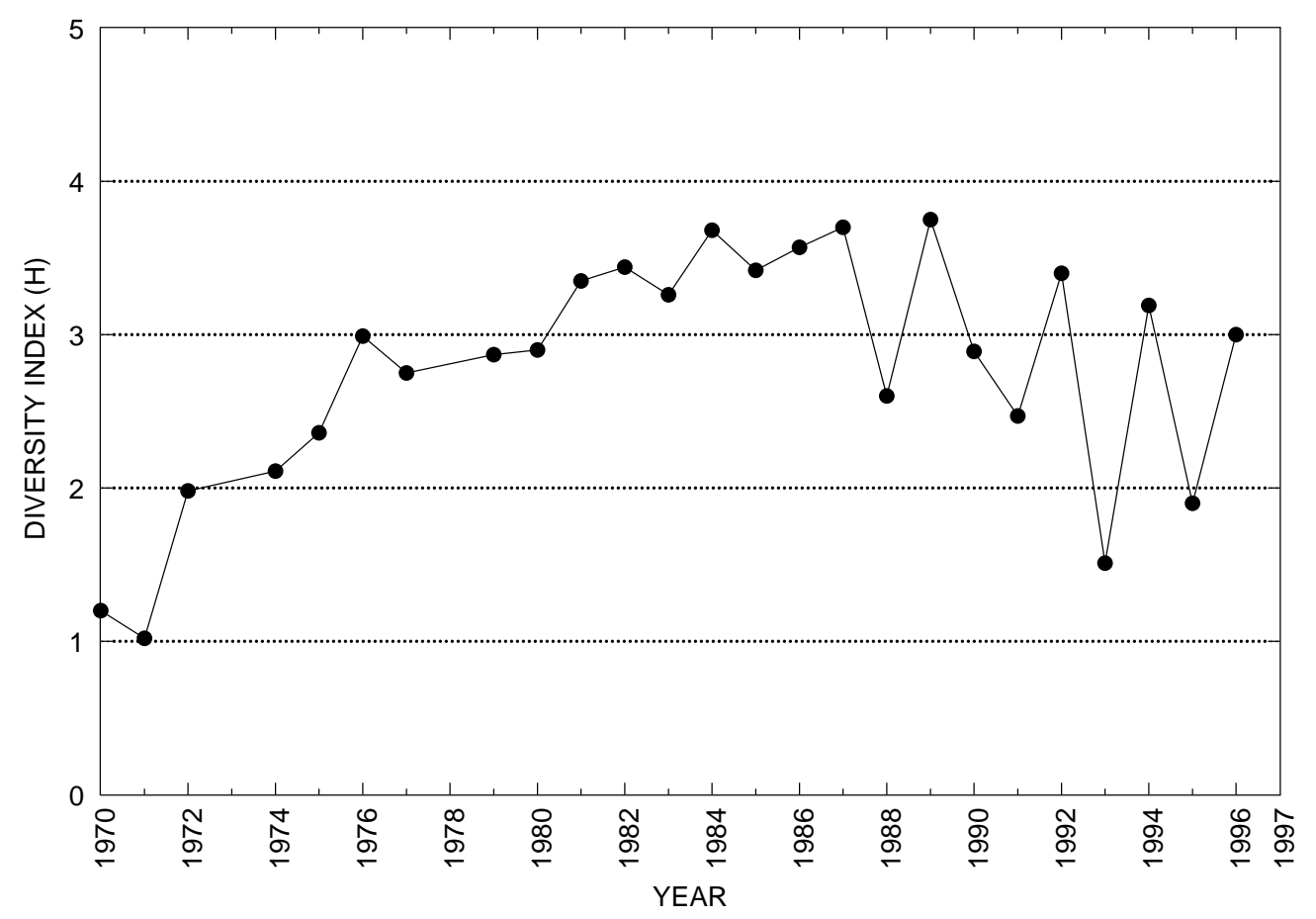


Table 6. Brillouin's diversity index, maximum diversity, minimum diversity, and relative evenness by site-Continued

$[-$, no data $]$

01475840 (Site 19) - Crum Creek at Whitehorse, Pa.

\begin{tabular}{ccccccc}
\hline Year & $\begin{array}{c}\text { Total } \\
\text { organisms }\end{array}$ & $\begin{array}{c}\text { Total } \\
\text { taxa }\end{array}$ & $\begin{array}{c}\text { Brillouin's } \\
\text { diversity } \\
\text { index }(\mathrm{H})\end{array}$ & $\begin{array}{c}\text { Maximum } \\
\text { diversity } \\
\left(\mathrm{H}_{\text {max }}\right)\end{array}$ & $\begin{array}{c}\text { Minimum } \\
\text { diversity } \\
\left(\mathrm{H}_{\text {min }}\right)\end{array}$ & $\begin{array}{c}\text { Evenness } \\
(\mathrm{E})\end{array}$ \\
\hline 1995 & 603 & 33 & 2.85 & 4.93 & 0.49 & 0.53 \\
1996 & 886 & 29 & 2.75 & 4.83 & .31 & .54 \\
1997 & - & - & - & - & - & - \\
\hline
\end{tabular}

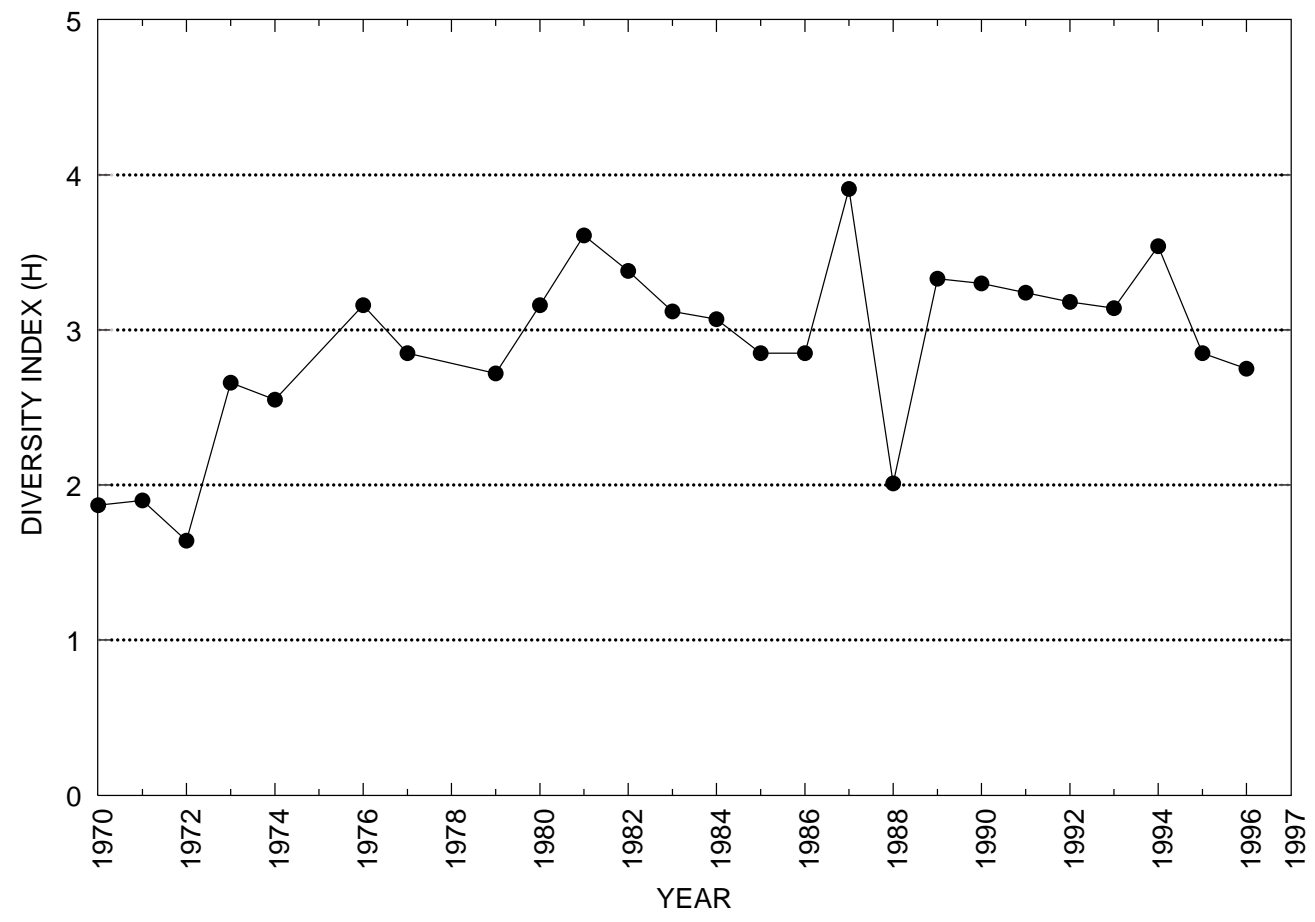


Table 6. Brillouin's diversity index, maximum diversity, minimum diversity, and relative evenness by site-Continued

$[-$, no data $]$

01476430 (Site 20) - Ridley Creek at Goshenville, Pa.

\begin{tabular}{|c|c|c|c|c|c|c|}
\hline Year & $\begin{array}{c}\text { Total } \\
\text { organisms }\end{array}$ & $\begin{array}{l}\text { Total } \\
\text { taxa }\end{array}$ & $\begin{array}{l}\text { Brillouin's } \\
\text { diversity } \\
\text { index }(\mathrm{H})\end{array}$ & $\begin{array}{c}\text { Maximum } \\
\text { diversity } \\
\left(\mathrm{H}_{\max }\right)\end{array}$ & $\begin{array}{c}\text { Minimum } \\
\text { diversity } \\
\left(\mathrm{H}_{\text {min }}\right)\end{array}$ & $\begin{array}{c}\text { Evenness } \\
\text { (E) }\end{array}$ \\
\hline 1995 & 412 & 25 & 3.56 & 4.57 & 0.50 & 0.75 \\
\hline 1996 & 201 & 19 & 3.17 & 4.17 & .68 & .71 \\
\hline 1997 & - & - & - & - & - & - \\
\hline
\end{tabular}

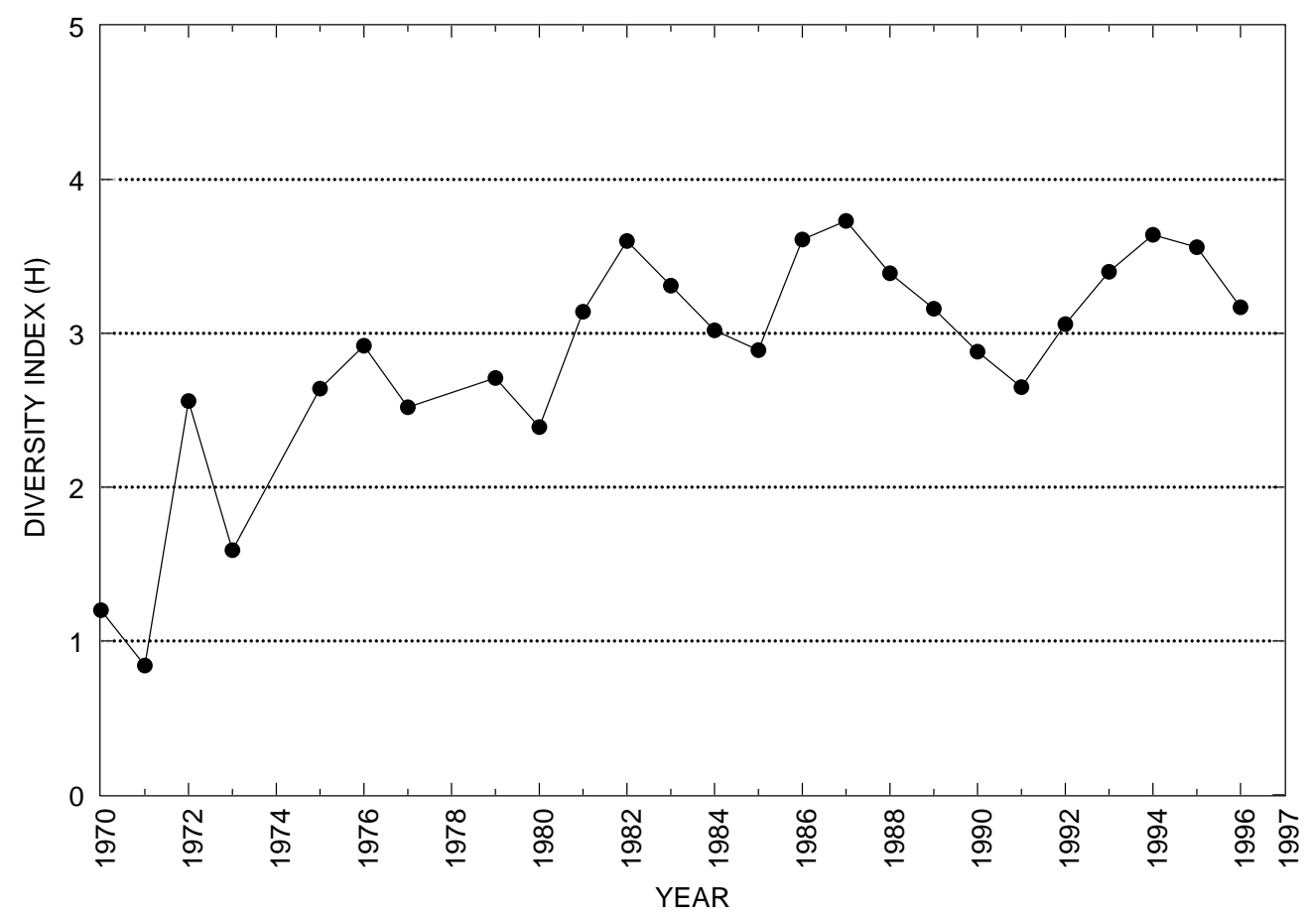


Table 6. Brillouin's diversity index, maximum diversity, minimum diversity, and relative evenness by site-Continued

$[-$, no data $]$

01476435 (Site 21) - Ridley Creek at Dutton Mill near West Chester, Pa.

\begin{tabular}{ccccccc}
\hline Year & $\begin{array}{c}\text { Total } \\
\text { organisms }\end{array}$ & $\begin{array}{c}\text { Total } \\
\text { taxa }\end{array}$ & $\begin{array}{c}\text { Brillouin's } \\
\text { diversity } \\
\text { index }(\mathrm{H})\end{array}$ & $\begin{array}{c}\text { Maximum } \\
\text { diversity } \\
\left(\mathrm{H}_{\text {max }}\right)\end{array}$ & $\begin{array}{c}\text { Minimum } \\
\text { diversity } \\
\left(\mathrm{H}_{\text {min }}\right)\end{array}$ & $\begin{array}{c}\text { Evenness } \\
(\mathrm{E})\end{array}$ \\
\hline 1995 & 915 & 36 & 3.51 & 5.11 & 0.38 & 0.66 \\
1996 & 633 & 34 & 3.43 & 5.05 & .48 & .65 \\
1997 & - & - & - & - & - & - \\
\hline
\end{tabular}

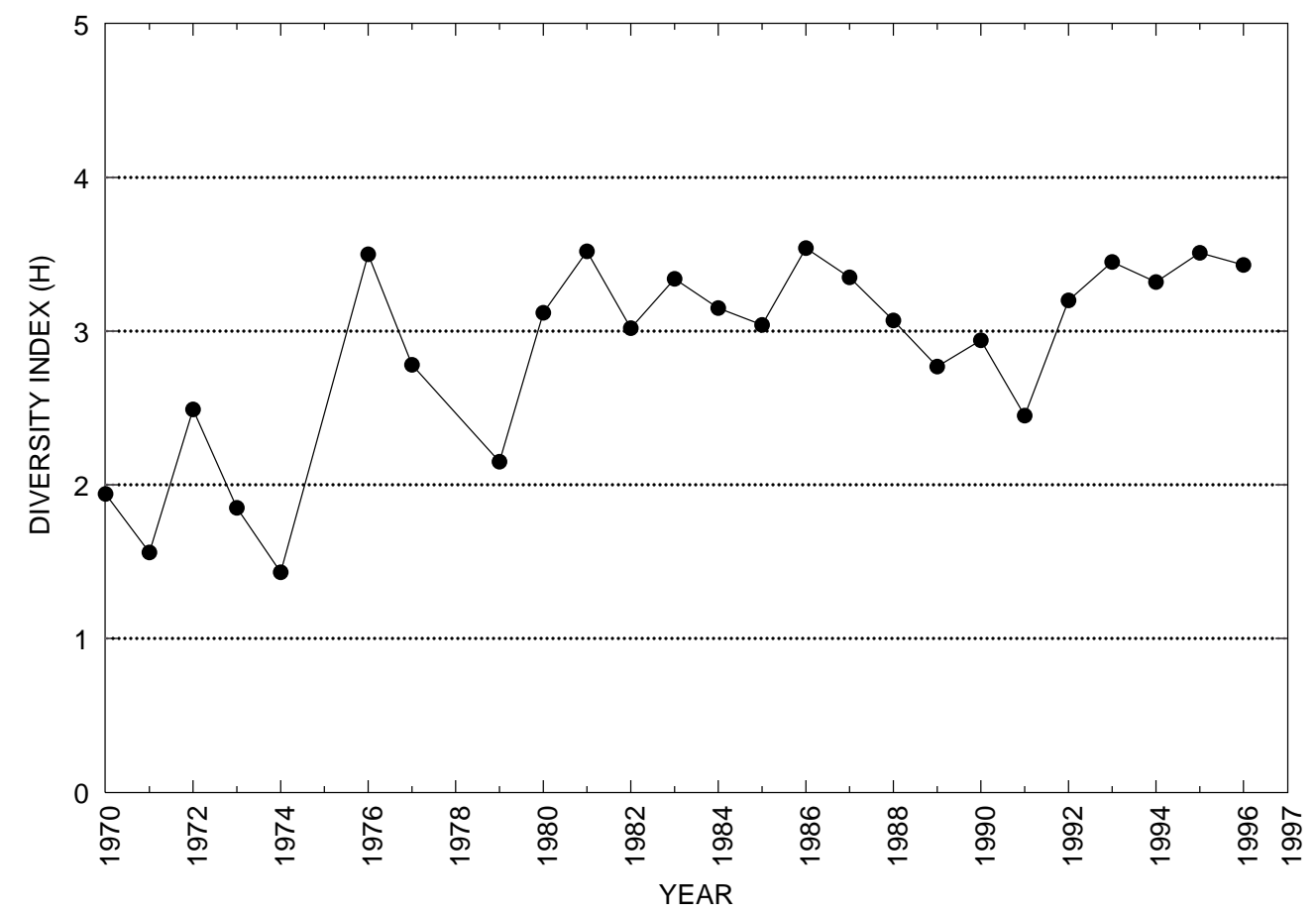


Table 6. Brillouin's diversity index, maximum diversity, minimum diversity, and relative evenness by site-Continued

$[-$, no data $]$

01476790 (Site 22) - East Branch Chester Creek at Green Hill, Pa.

\begin{tabular}{ccccccc}
\hline Year & $\begin{array}{c}\text { Total } \\
\text { organisms }\end{array}$ & $\begin{array}{c}\text { Total } \\
\text { taxa }\end{array}$ & $\begin{array}{c}\text { Brillouin's } \\
\text { diversity } \\
\text { index }(\mathrm{H})\end{array}$ & $\begin{array}{c}\text { Maximum } \\
\text { diversity } \\
\left(\mathrm{H}_{\text {max }}\right)\end{array}$ & $\begin{array}{c}\text { Minimum } \\
\text { diversity } \\
\left(\mathrm{H}_{\text {min }}\right)\end{array}$ & $\begin{array}{c}\text { Evenness } \\
(\mathrm{E})\end{array}$ \\
\hline 1995 & 331 & 22 & 2.77 & 4.27 & 0.53 & 0.60 \\
1996 & - & - & - & - & - & - \\
1997 & 250 & 17 & 3.17 & 4.07 & .51 & .75 \\
\hline
\end{tabular}

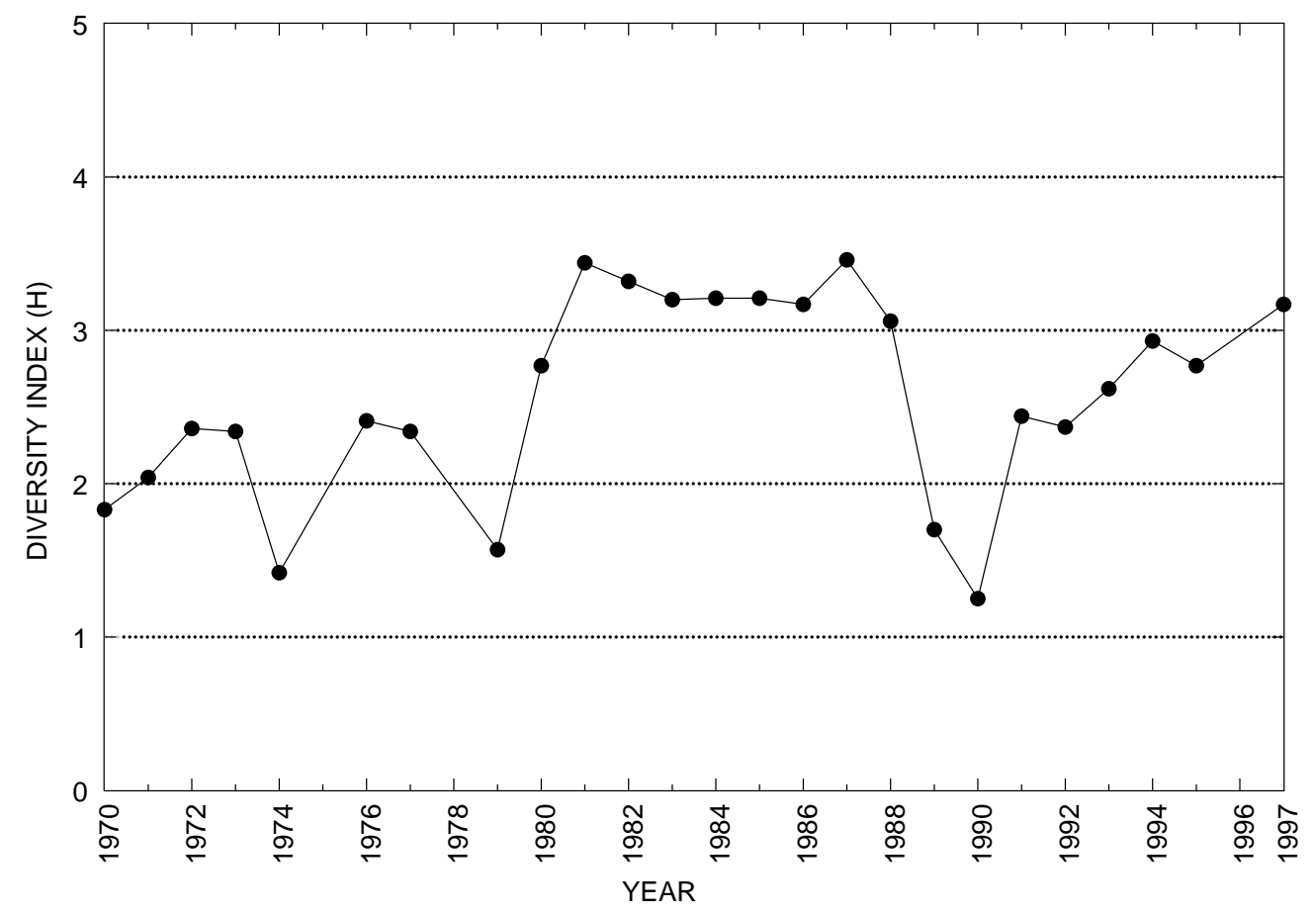


Table 6. Brillouin's diversity index, maximum diversity, minimum diversity, and relative evenness by site-Continued

$[-$, no data $]$

01476830 (Site 23) - East Branch Chester Creek at Milltown, Pa.

\begin{tabular}{|c|c|c|c|c|c|c|}
\hline Year & $\begin{array}{c}\text { Total } \\
\text { organisms }\end{array}$ & $\begin{array}{l}\text { Total } \\
\text { taxa }\end{array}$ & $\begin{array}{l}\text { Brillouin's } \\
\text { diversity } \\
\text { index }(\mathrm{H})\end{array}$ & $\begin{array}{c}\text { Maximum } \\
\text { diversity } \\
\left(\mathrm{H}_{\max }\right)\end{array}$ & $\begin{array}{l}\text { Minimum } \\
\text { diversity } \\
\left(\mathrm{H}_{\mathrm{min}}\right)\end{array}$ & $\begin{array}{c}\text { Evenness } \\
(\mathrm{E})\end{array}$ \\
\hline 1995 & 281 & 25 & 3.12 & 4.46 & 0.69 & 0.64 \\
\hline 1996 & 1,594 & 29 & 3.64 & 4.89 & .19 & .73 \\
\hline 1997 & - & - & - & - & - & - \\
\hline
\end{tabular}

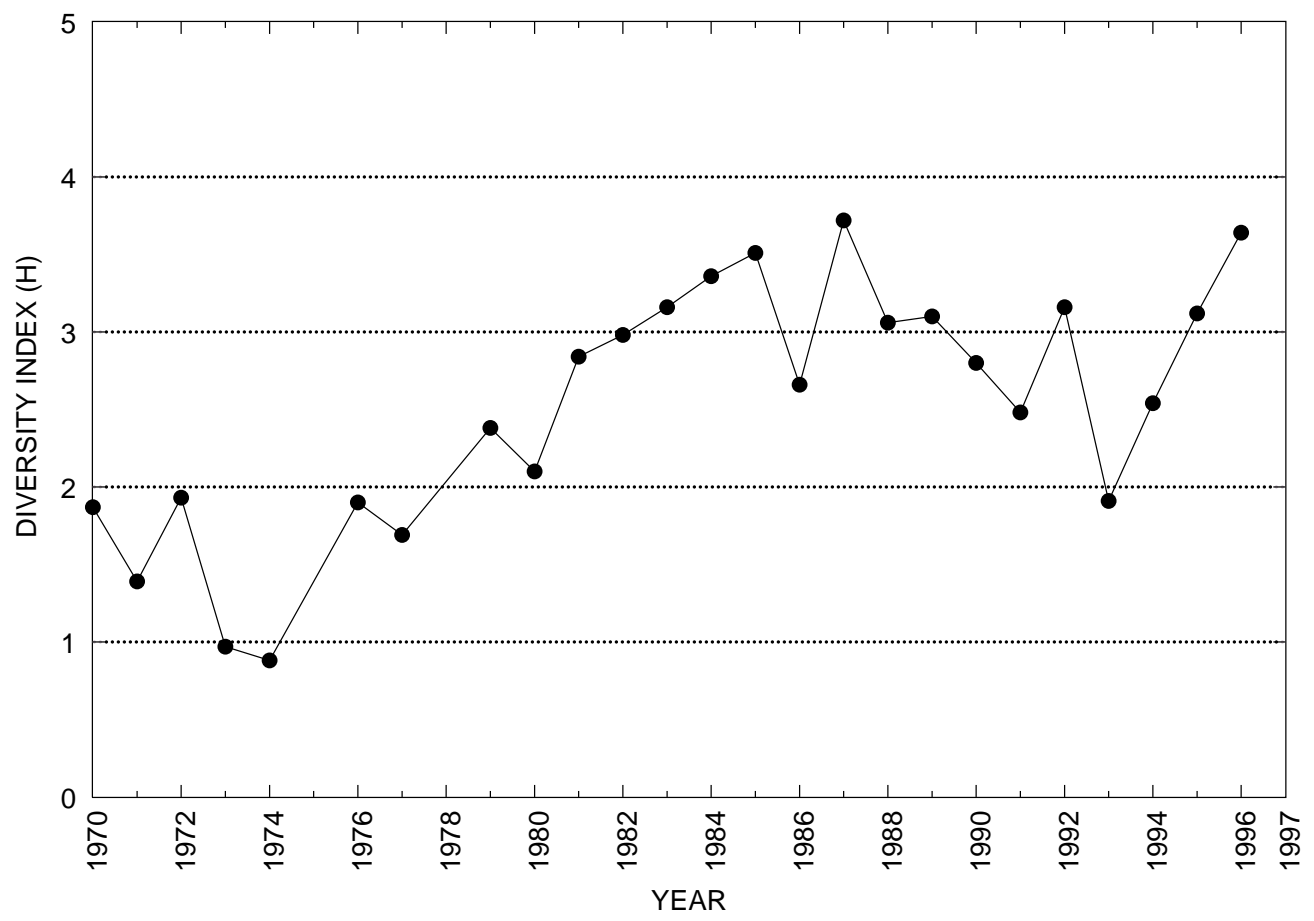


Table 6. Brillouin's diversity index, maximum diversity, minimum diversity, and relative evenness by site-Continued

01476835 (Site 24) - East Branch Chester Creek at Westtown, Pa.

\begin{tabular}{ccccccc}
\hline Year & $\begin{array}{c}\text { Total } \\
\text { organisms }\end{array}$ & $\begin{array}{c}\text { Total } \\
\text { taxa }\end{array}$ & $\begin{array}{c}\text { Brillouin's } \\
\text { diversity } \\
\text { index }(\mathrm{H})\end{array}$ & $\begin{array}{c}\text { Maximum } \\
\text { diversity } \\
\left(\mathrm{H}_{\text {max }}\right)\end{array}$ & $\begin{array}{c}\text { Minimum } \\
\text { diversity } \\
\left(\mathrm{H}_{\text {min }}\right)\end{array}$ & $\begin{array}{c}\text { Evenness } \\
(\mathrm{E})\end{array}$ \\
\hline 1995 & 464 & 26 & 3.41 & 4.72 & 0.48 & 0.69 \\
1996 & 1,386 & 30 & 3.45 & 4.85 & .22 & .70 \\
1997 & 1,291 & 26 & 3.36 & 4.70 & .20 & .70 \\
\hline
\end{tabular}

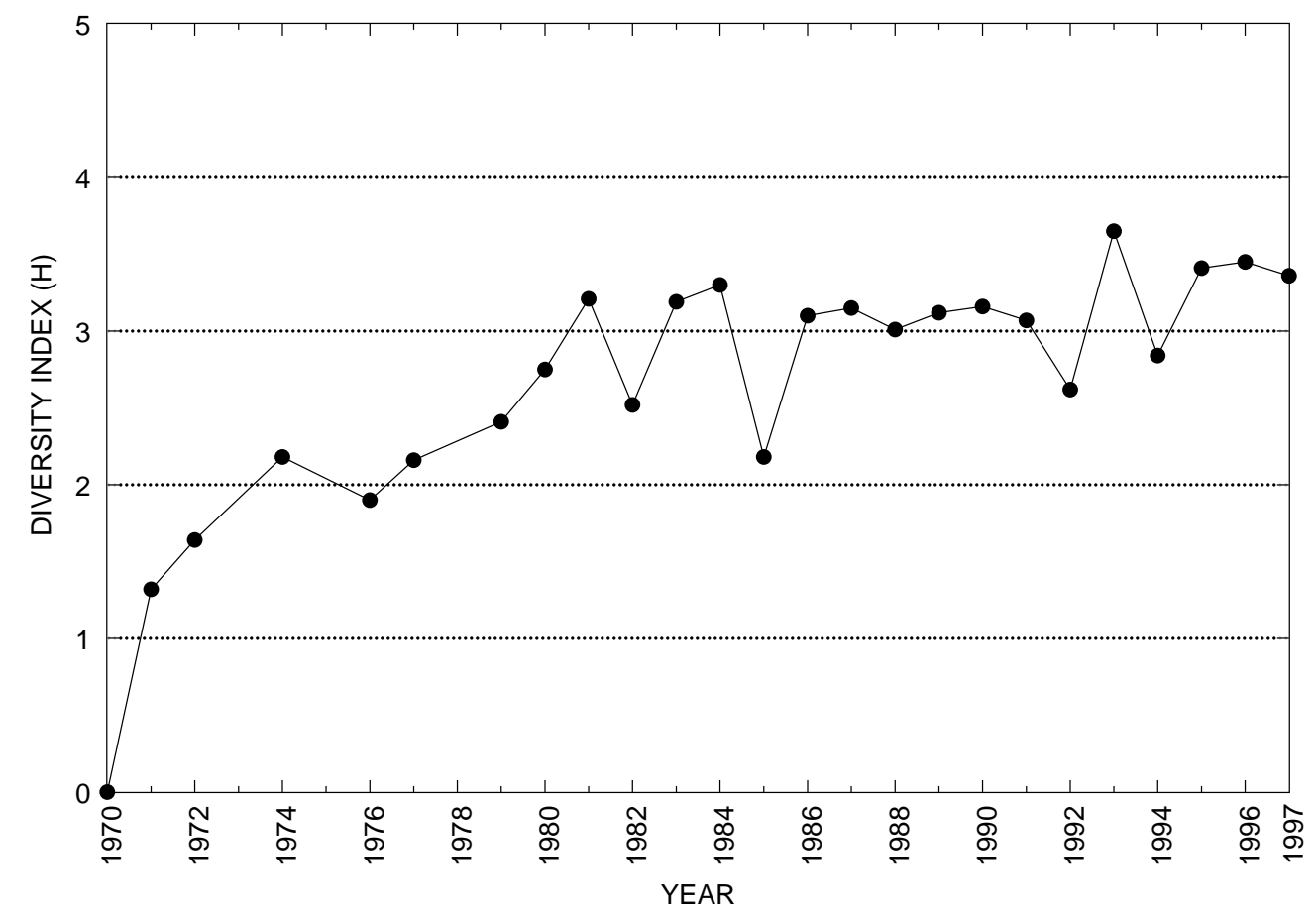


Table 6. Brillouin's diversity index, maximum diversity, minimum diversity, and relative evenness by site-Continued

01476840 (Site 25) - Goose Creek Tributary to East Branch Chester Creek near West Chester, $\mathrm{Pa}$.

\begin{tabular}{ccccccc}
\hline Year & $\begin{array}{c}\text { Total } \\
\text { organisms }\end{array}$ & $\begin{array}{c}\text { Total } \\
\text { taxa }\end{array}$ & $\begin{array}{c}\text { Brillouin's } \\
\text { diversity } \\
\text { index }(\mathrm{H})\end{array}$ & $\begin{array}{c}\text { Maximum } \\
\text { diversity } \\
\left(\mathrm{H}_{\text {max }}\right)\end{array}$ & $\begin{array}{c}\text { Minimum } \\
\text { diversity } \\
\left(\mathrm{H}_{\text {min }}\right)\end{array}$ & $\begin{array}{c}\text { Evenness } \\
(\mathrm{E})\end{array}$ \\
\hline 1995 & 1,868 & 18 & 2.12 & 4.18 & 0.10 & 0.50 \\
1996 & 1,298 & 19 & 2.58 & 4.22 & .14 & .60 \\
1997 & 1,320 & 12 & 2.38 & 3.55 & .09 & .66 \\
\hline
\end{tabular}

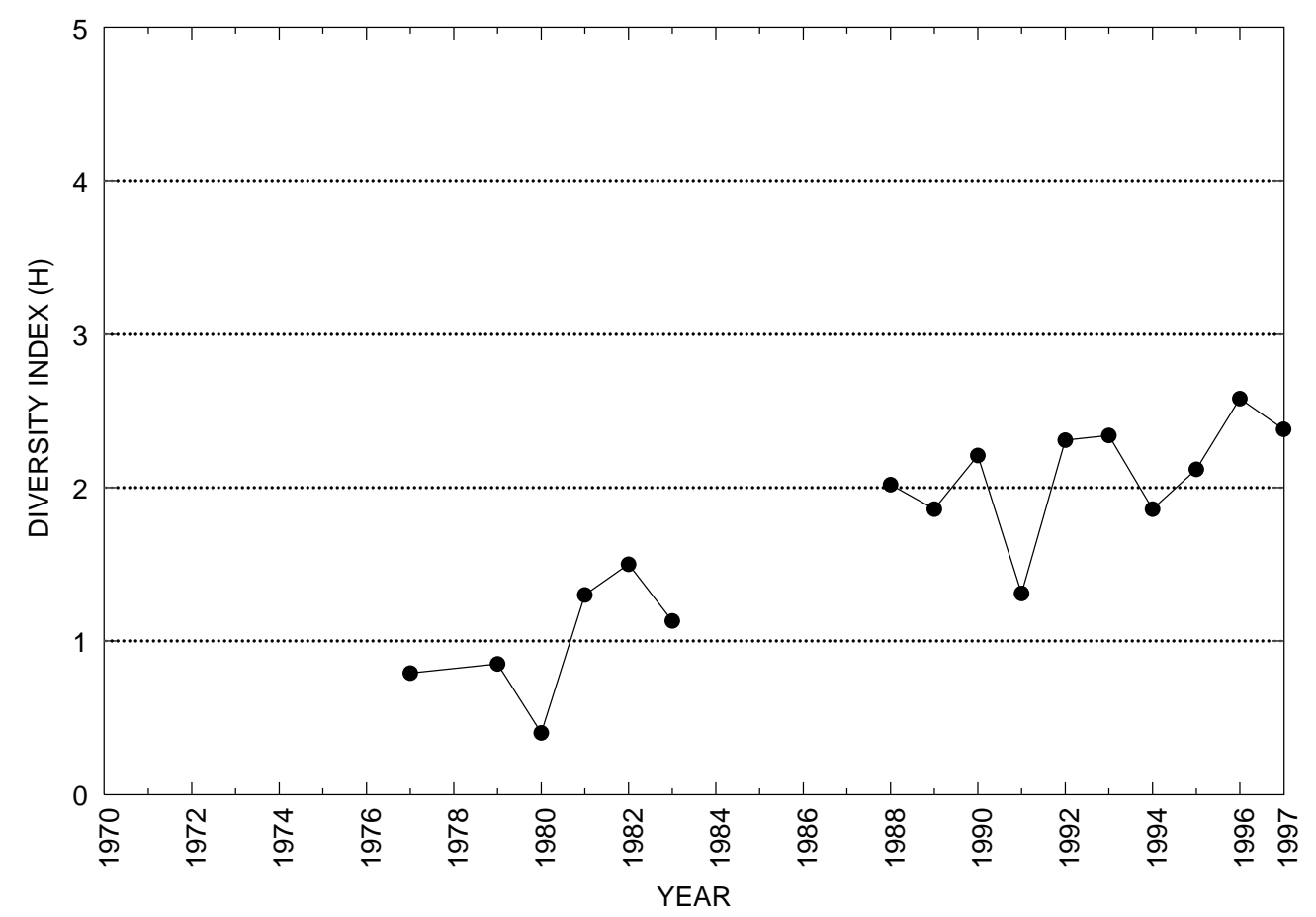


Table 6. Brillouin's diversity index, maximum diversity, minimum diversity, and relative evenness by site-Continued

[-, no data]

01476848 (Site 51) - East Branch Chester Creek Below Goose Creek near

West Chester, Pa.

\begin{tabular}{ccccccc}
\hline Year & $\begin{array}{c}\text { Total } \\
\text { organisms }\end{array}$ & $\begin{array}{c}\text { Total } \\
\text { taxa }\end{array}$ & $\begin{array}{c}\text { Brillouin's } \\
\text { diversity } \\
\text { index }(\mathrm{H})\end{array}$ & $\begin{array}{c}\text { Maximum } \\
\text { diversity } \\
\left(\mathrm{H}_{\text {max }}\right)\end{array}$ & $\begin{array}{c}\text { Minimum } \\
\text { diversity } \\
\left(\mathrm{H}_{\text {min }}\right)\end{array}$ & $\begin{array}{c}\text { Evenness } \\
(\mathrm{E})\end{array}$ \\
\hline 1995 & 589 & 22 & 3.04 & 4.47 & 0.33 & 0.65 \\
1996 & - & - & - & - & - & - \\
1997 & 2,219 & 18 & 2.63 & 4.45 & .15 & .56 \\
\hline
\end{tabular}

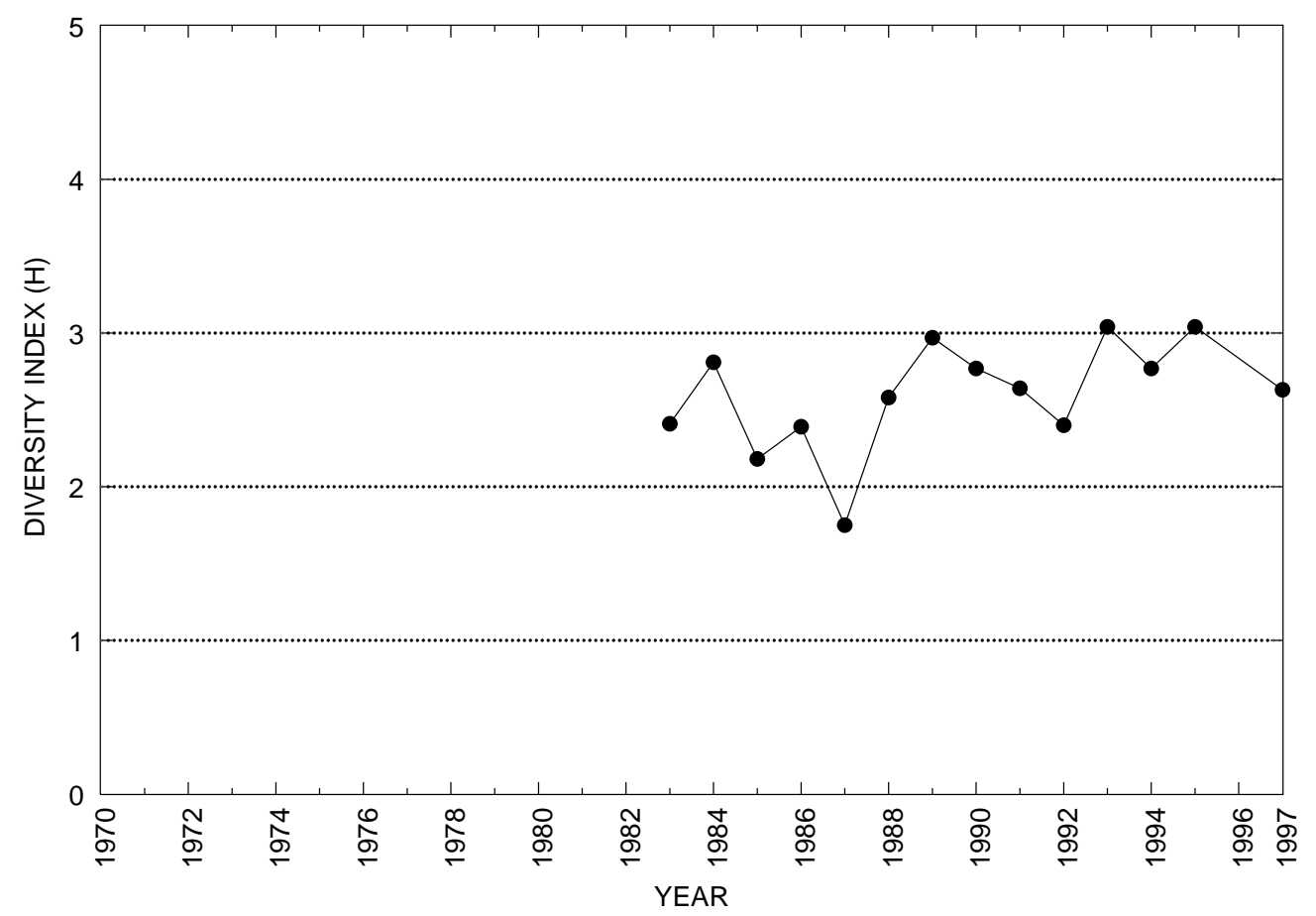


Table 6. Brillouin's diversity index, maximum diversity, minimum diversity, and relative evenness by site-Continued

[-, no data]

01478120 (Site 28) - East Branch White Clay Creek at Avondale, Pa.

\begin{tabular}{ccccccc}
\hline Year & $\begin{array}{c}\text { Total } \\
\text { organisms }\end{array}$ & $\begin{array}{c}\text { Total } \\
\text { taxa }\end{array}$ & $\begin{array}{c}\text { Brillouin's } \\
\text { diversity } \\
\text { index }(\mathrm{H})\end{array}$ & $\begin{array}{c}\text { Maximum } \\
\text { diversity } \\
\left(\mathrm{H}_{\text {max }}\right)\end{array}$ & $\begin{array}{c}\text { Minimum } \\
\text { diversity } \\
\left(\mathrm{H}_{\text {min }}\right)\end{array}$ & $\begin{array}{c}\text { Evenness } \\
(\mathrm{E})\end{array}$ \\
\hline 1995 & 1,543 & 28 & 2.71 & 4.75 & 0.19 & 0.55 \\
1996 & - & - & - & - & - & - \\
1997 & 1,717 & 24 & 2.87 & 4.57 & .14 & .61 \\
\hline
\end{tabular}

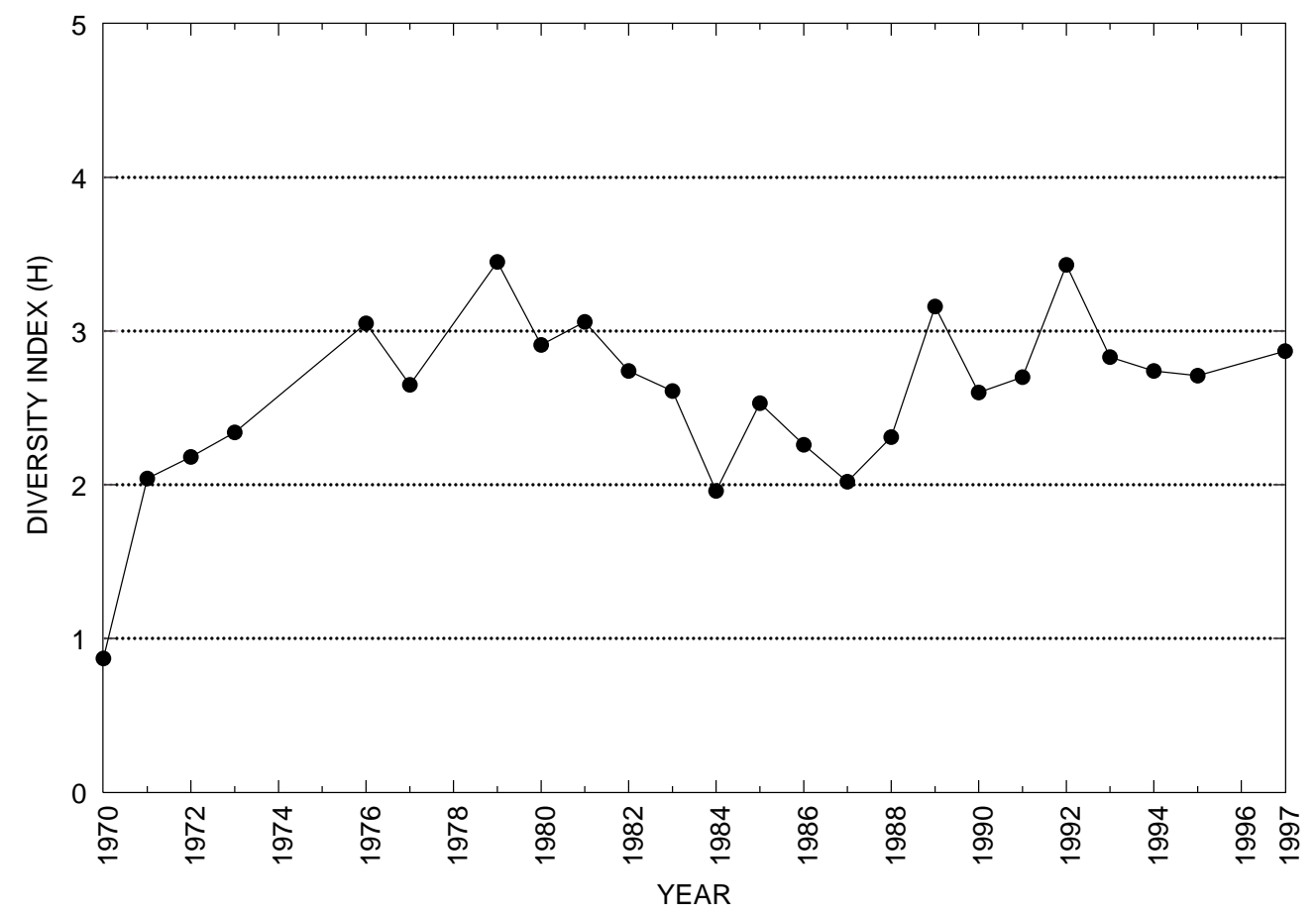


Table 6. Brillouin's diversity index, maximum diversity, minimum diversity, and relative evenness by site-Continued

[-, no data]

01478190 (Site 29) - Middle Branch White Clay Creek at Wickerton, Pa.

\begin{tabular}{ccccccc}
\hline Year & $\begin{array}{c}\text { Total } \\
\text { organisms }\end{array}$ & $\begin{array}{c}\text { Total } \\
\text { taxa }\end{array}$ & $\begin{array}{c}\text { Brillouin's } \\
\text { diversity } \\
\text { index }(\mathrm{H})\end{array}$ & $\begin{array}{c}\text { Maximum } \\
\text { diversity } \\
\left(\mathrm{H}_{\text {max }}\right)\end{array}$ & $\begin{array}{c}\text { Minimum } \\
\text { diversity } \\
\left(\mathrm{H}_{\text {min }}\right)\end{array}$ & $\begin{array}{c}\text { Evenness } \\
(\mathrm{E})\end{array}$ \\
\hline 1995 & 1,841 & 28 & 2.26 & 4.81 & 0.18 & 0.45 \\
1996 & - & - & - & - & - & - \\
1997 & 1,371 & 29 & 3.10 & 4.81 & .21 & .63 \\
\hline
\end{tabular}

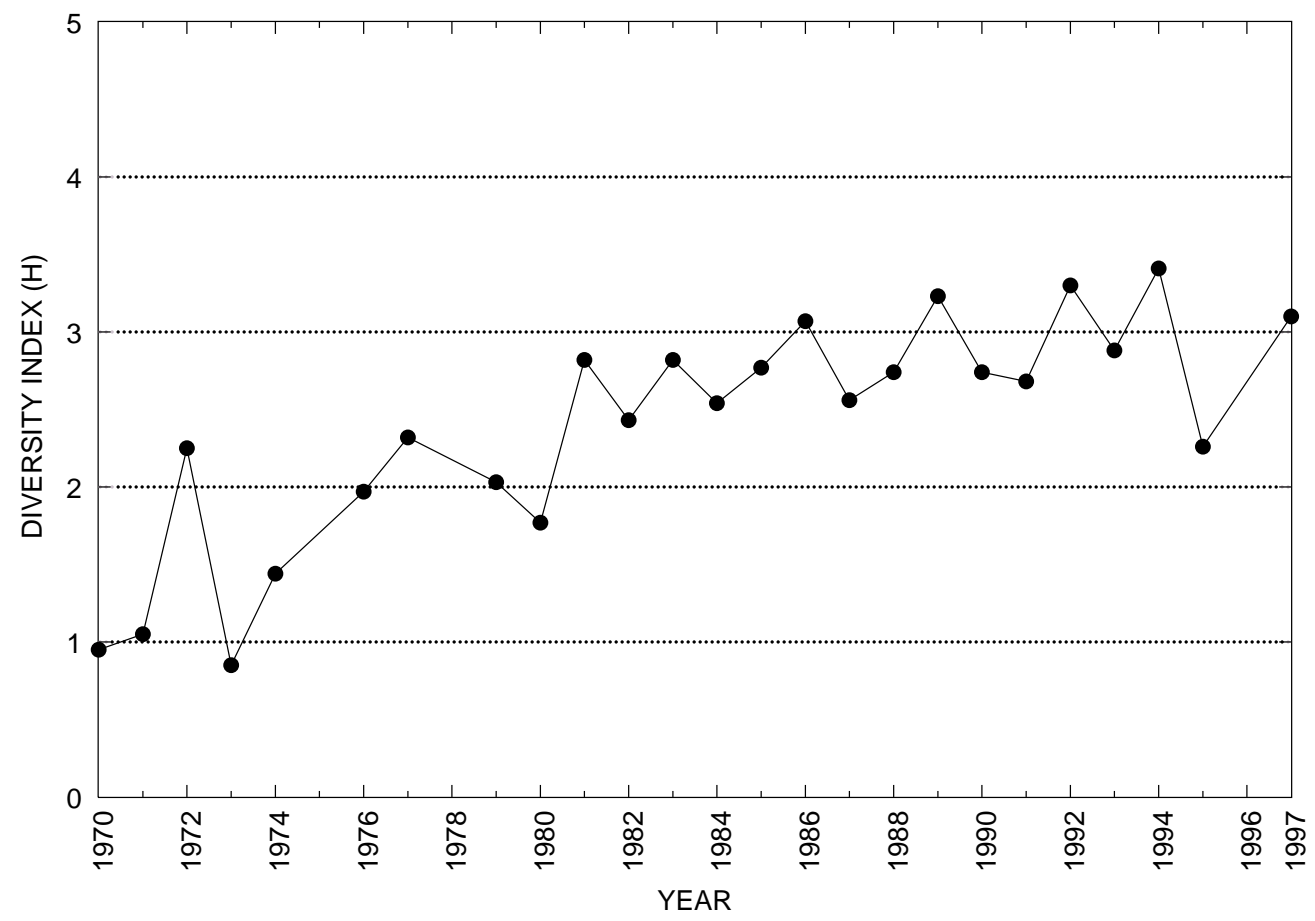


Table 6. Brillouin's diversity index, maximum diversity, minimum diversity, and relative evenness by site-Continued

[-, no data]

01478220 (Site 30) - West Branch White Clay Creek near Chesterville, Pa.

\begin{tabular}{ccccccc}
\hline Year & $\begin{array}{c}\text { Total } \\
\text { organisms }\end{array}$ & $\begin{array}{c}\text { Total } \\
\text { taxa }\end{array}$ & $\begin{array}{c}\text { Brillouin's } \\
\text { diversity } \\
\text { index }(\mathrm{H})\end{array}$ & $\begin{array}{c}\text { Maximum } \\
\text { diversity } \\
\left(\mathrm{H}_{\text {max }}\right)\end{array}$ & $\begin{array}{c}\text { Minimum } \\
\text { diversity } \\
\left(\mathrm{H}_{\text {min }}\right)\end{array}$ & $\begin{array}{c}\text { Evenness } \\
(\mathrm{E})\end{array}$ \\
\hline 1995 & 637 & 30 & 3.26 & 4.80 & 0.42 & 0.65 \\
1996 & - & - & - & - & - & - \\
1997 & 1,470 & 27 & 2.95 & 4.84 & .22 & .59 \\
\hline
\end{tabular}

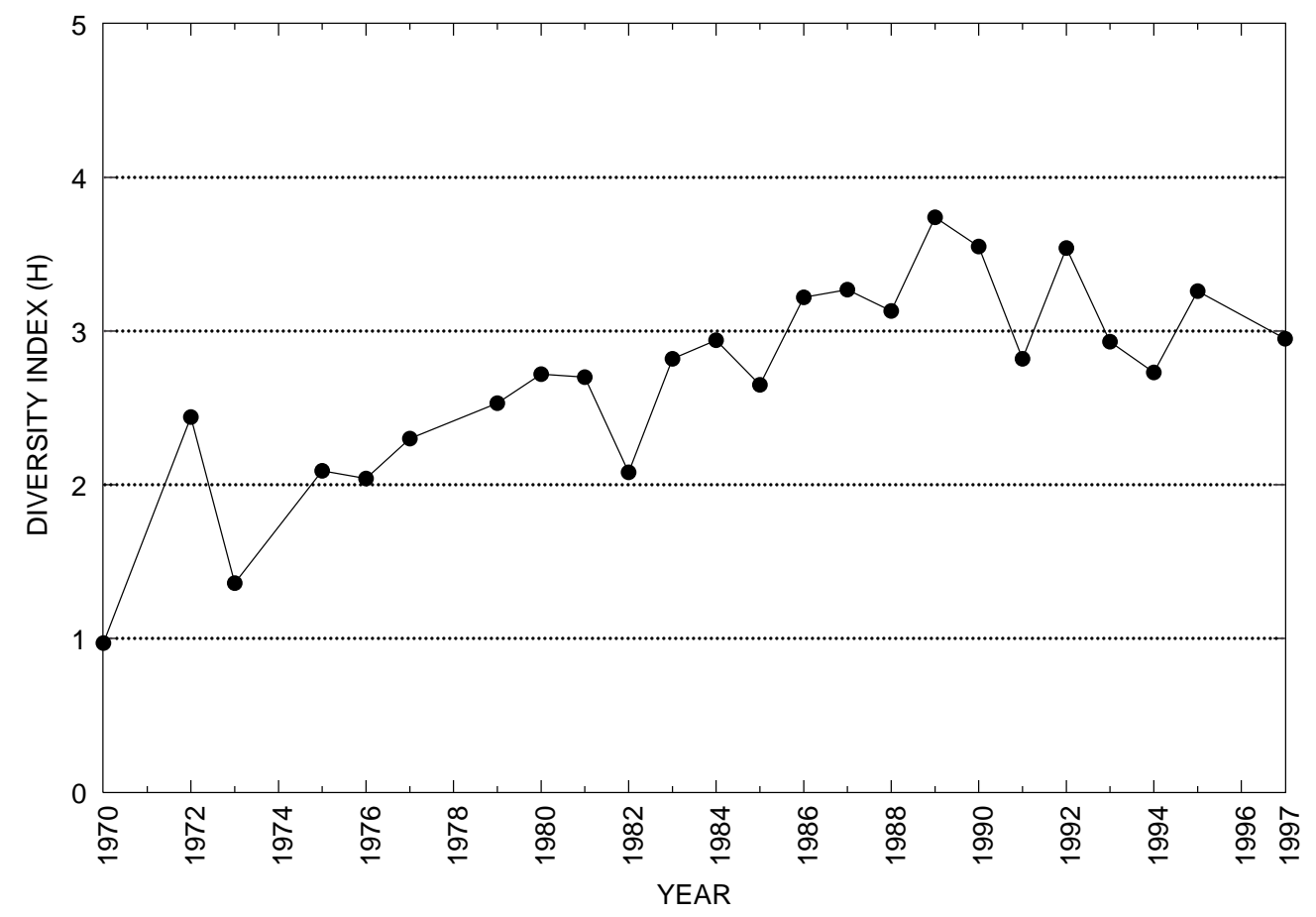


Table 6. Brillouin's diversity index, maximum diversity, minimum diversity, and relative evenness by site-Continued

01479680 (Site 27) - West Branch Red Clay Creek at Kennett Square, Pa.

\begin{tabular}{ccccccc}
\hline Year & $\begin{array}{c}\text { Total } \\
\text { organisms }\end{array}$ & $\begin{array}{c}\text { Total } \\
\text { taxa }\end{array}$ & $\begin{array}{c}\text { Brillouin's } \\
\text { diversity } \\
\text { index }(\mathrm{H})\end{array}$ & $\begin{array}{c}\text { Maximum } \\
\text { diversity } \\
\left(\mathrm{H}_{\text {max }}\right)\end{array}$ & $\begin{array}{c}\text { Minimum } \\
\text { diversity } \\
\left(\mathrm{H}_{\text {min }}\right)\end{array}$ & $\begin{array}{c}\text { Evenness } \\
(\mathrm{E})\end{array}$ \\
\hline 1995 & 323 & 22 & 3.12 & 4.43 & 0.54 & 0.66 \\
1996 & 178 & 15 & 2.17 & 3.93 & .58 & .47 \\
1997 & 790 & 26 & 2.86 & 4.65 & .30 & .59 \\
\hline
\end{tabular}

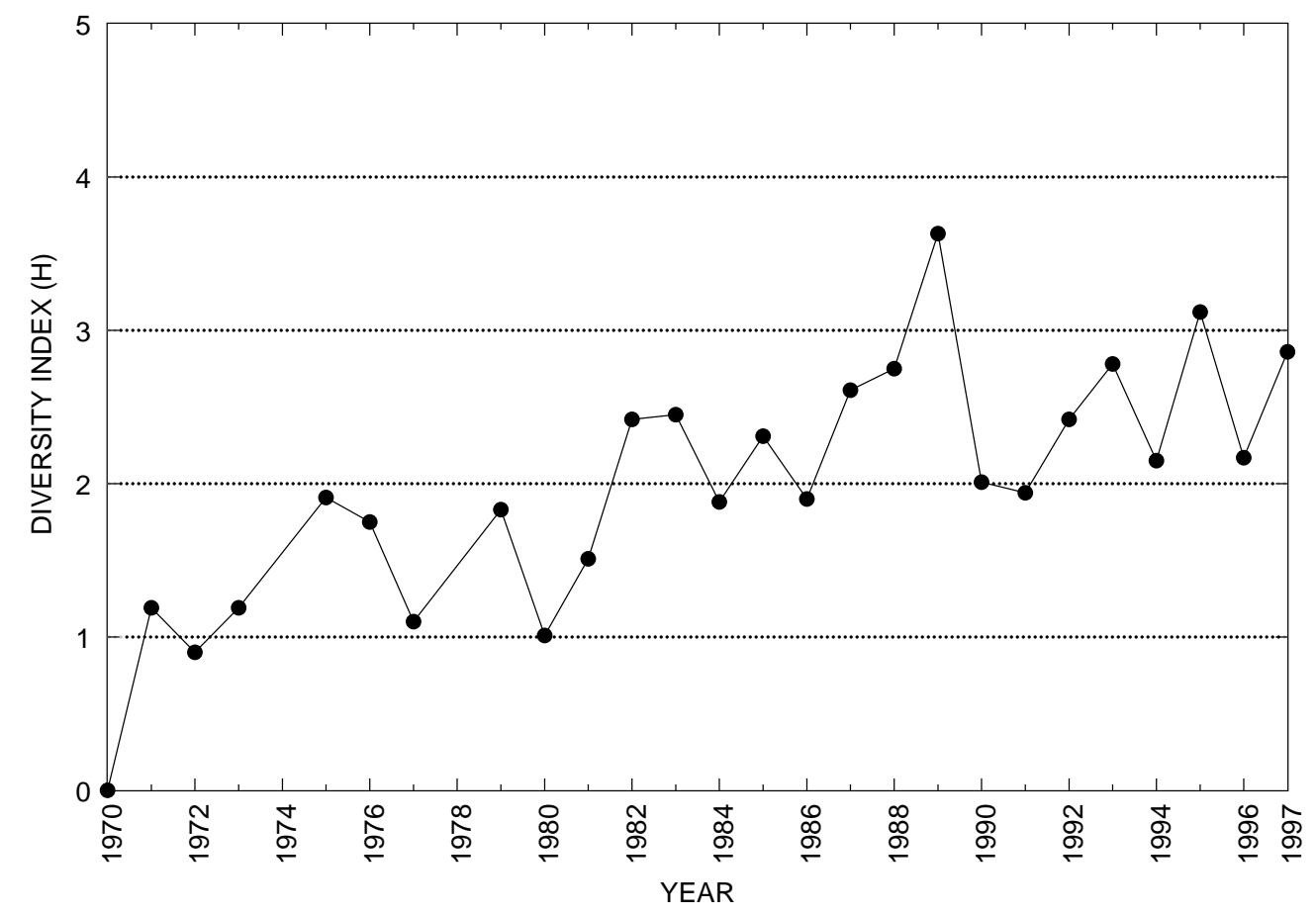


Table 6. Brillouin's diversity index, maximum diversity, minimum diversity, and relative evenness by site-Continued

01479800 (Site 26) - East Branch Red Clay Creek near Five Point, Pa.

\begin{tabular}{ccccccc}
\hline Year & $\begin{array}{c}\text { Total } \\
\text { organisms }\end{array}$ & $\begin{array}{c}\text { Total } \\
\text { taxa }\end{array}$ & $\begin{array}{c}\text { Brillouin's } \\
\text { diversity } \\
\text { index }(\mathrm{H})\end{array}$ & $\begin{array}{c}\text { Maximum } \\
\text { diversity } \\
\left(\mathrm{H}_{\text {max }}\right)\end{array}$ & $\begin{array}{c}\text { Minimum } \\
\text { diversity } \\
\left(\mathrm{H}_{\text {min }}\right)\end{array}$ & $\begin{array}{c}\text { Evenness } \\
(\mathrm{E})\end{array}$ \\
\hline 1995 & 514 & 22 & 3.30 & 4.39 & 0.37 & 0.73 \\
1996 & 1,240 & 22 & 2.73 & 4.43 & .17 & .60 \\
1997 & 1,213 & 17 & 2.33 & 4.06 & .14 & .56 \\
\hline
\end{tabular}

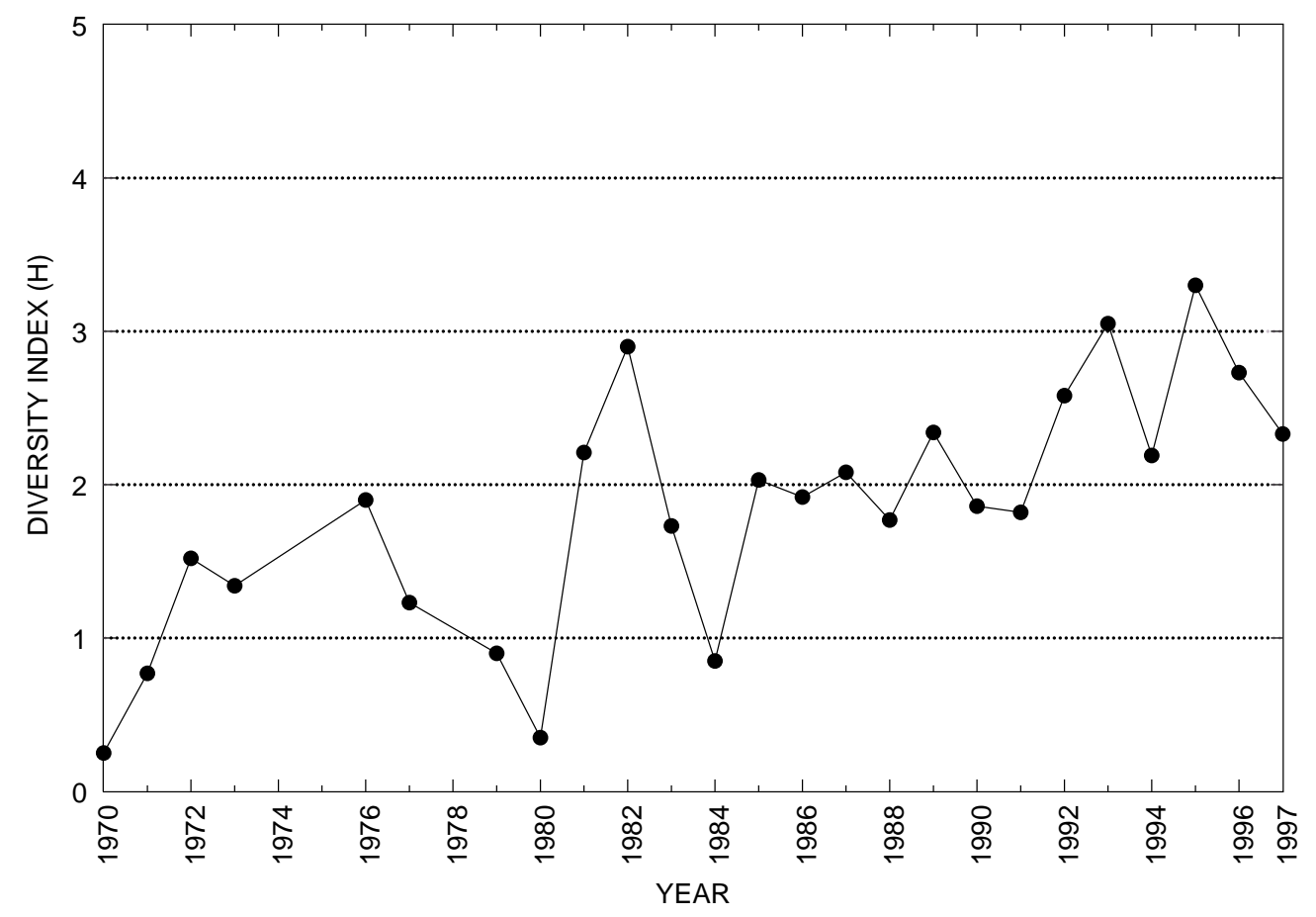


Table 6. Brillouin's diversity index, maximum diversity, minimum diversity, and relative evenness by siteContinued

[-, no data]

01480434 (Site 37) - West Branch Brandywine Creek at Rock Run, Pa.

\begin{tabular}{ccccccc}
\hline Year & $\begin{array}{c}\text { Total } \\
\text { organisms }\end{array}$ & $\begin{array}{c}\text { Total } \\
\text { taxa }\end{array}$ & $\begin{array}{c}\text { Brillouin's } \\
\text { diversity } \\
\text { index }(\mathrm{H})\end{array}$ & $\begin{array}{c}\text { Maximum } \\
\text { diversity } \\
\left(\mathrm{H}_{\text {max }}\right)\end{array}$ & $\begin{array}{c}\text { Minimum } \\
\text { diversity } \\
\left(\mathrm{H}_{\text {min }}\right)\end{array}$ & $\begin{array}{c}\text { Evenness } \\
(\mathrm{E})\end{array}$ \\
\hline 1995 & 440 & 36 & 3.73 & 4.99 & 0.69 & 0.71 \\
1996 & 511 & 27 & 3.43 & 4.79 & .46 & .69 \\
1997 & -- & -- & -- & -- & -- & -- \\
\hline
\end{tabular}

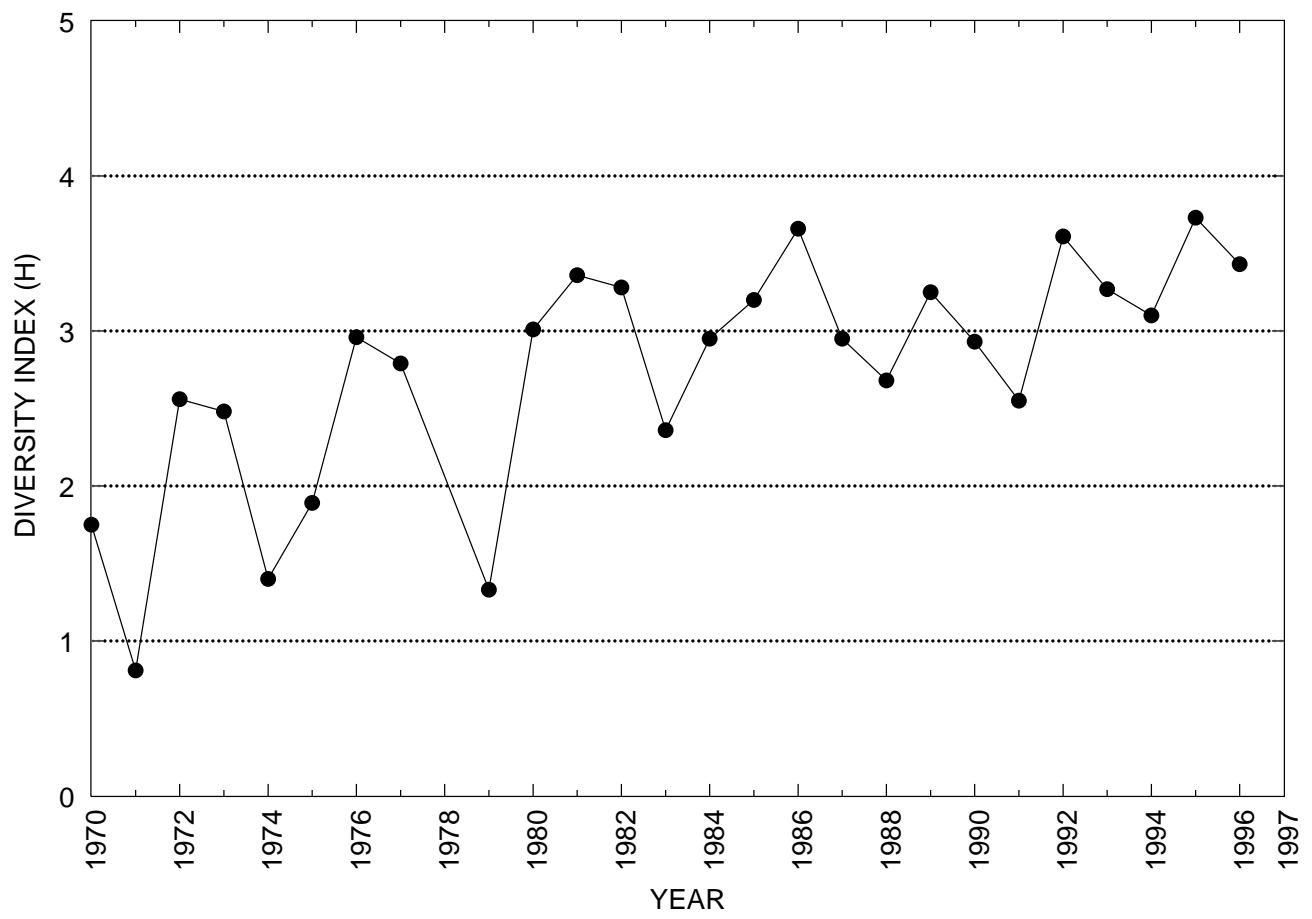


Table 6. Brillouin's diversity index, maximum diversity, minimum diversity, and relative evenness by site-Continued

[-, no data]

01480629 (Site 46) - Buck Run at Doe Run, Pa.

\begin{tabular}{ccccccc}
\hline Year & $\begin{array}{c}\text { Total } \\
\text { organisms }\end{array}$ & $\begin{array}{c}\text { Total } \\
\text { taxa }\end{array}$ & $\begin{array}{c}\text { Brillouin's } \\
\text { diversity } \\
\text { index }(\mathrm{H})\end{array}$ & $\begin{array}{c}\text { Maximum } \\
\text { diversity } \\
\left(\mathrm{H}_{\text {max }}\right)\end{array}$ & $\begin{array}{c}\text { Minimum } \\
\text { diversity } \\
\left(\mathrm{H}_{\text {min }}\right)\end{array}$ & $\begin{array}{c}\text { Evenness } \\
(\mathrm{E})\end{array}$ \\
\hline 1995 & 674 & 27 & 3.38 & 4.80 & 0.36 & 0.68 \\
1996 & - & - & - & - & - & - \\
1997 & 1,405 & 31 & 3.47 & 4.91 & .22 & .69 \\
\hline
\end{tabular}

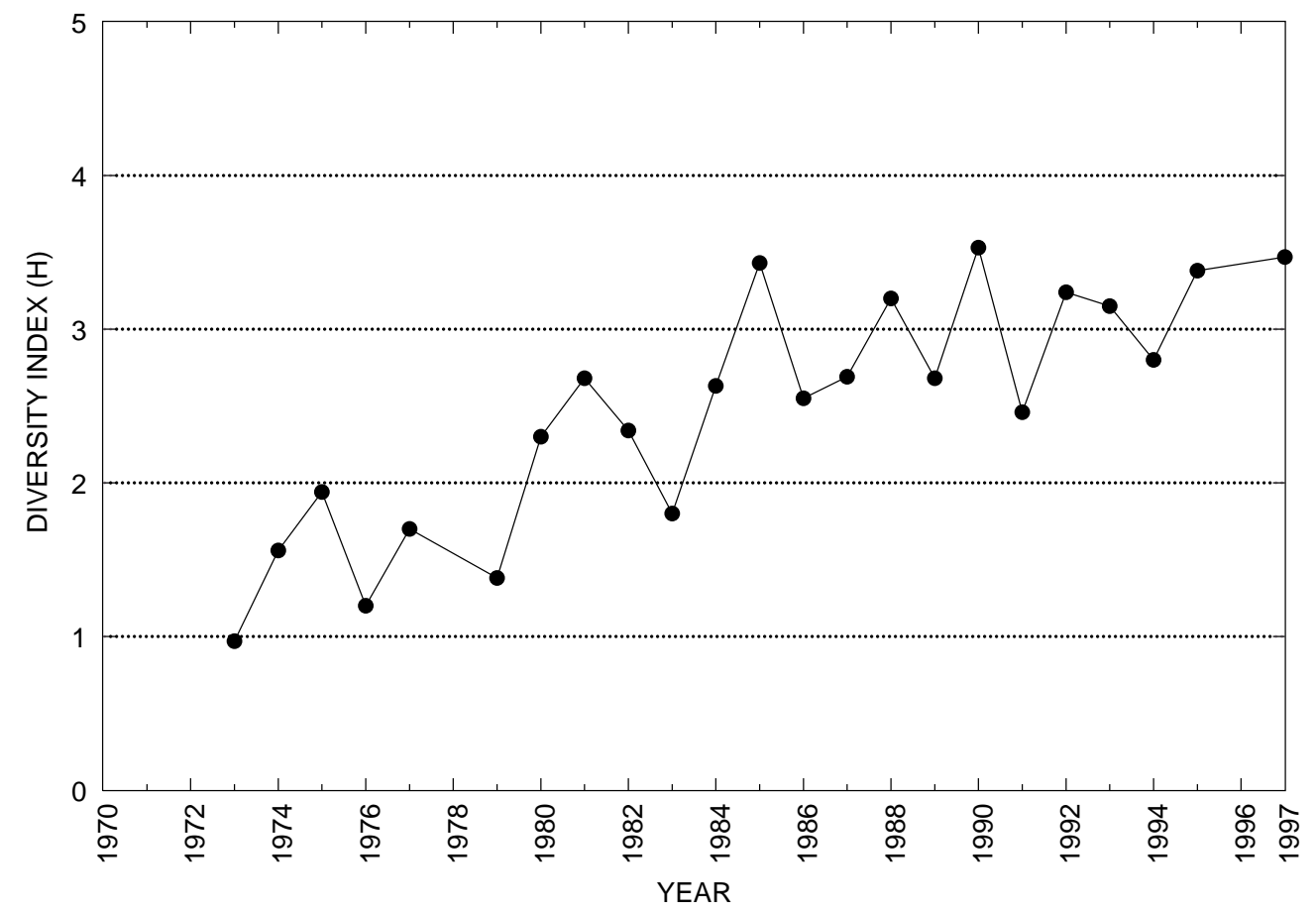


Table 6. Brillouin's diversity index, maximum diversity, minimum diversity, and relative evenness by site-Continued

[-, no data]

01480632 (Site 45) - Doe Run at Springdell, Pa.

\begin{tabular}{ccccccc}
\hline Year & $\begin{array}{c}\text { Total } \\
\text { organisms }\end{array}$ & $\begin{array}{c}\text { Total } \\
\text { taxa }\end{array}$ & $\begin{array}{c}\text { Brillouin's } \\
\text { diversity } \\
\text { index }(\mathrm{H})\end{array}$ & $\begin{array}{c}\text { Maximum } \\
\text { diversity } \\
\left(\mathrm{H}_{\text {max }}\right)\end{array}$ & $\begin{array}{c}\text { Minimum } \\
\text { diversity } \\
\left(\mathrm{H}_{\text {min }}\right)\end{array}$ & $\begin{array}{c}\text { Evenness } \\
(\mathrm{E})\end{array}$ \\
\hline 1995 & 820 & 32 & 3.60 & 4.98 & 0.36 & 0.70 \\
1996 & - & - & - & - & - & - \\
1997 & 1,078 & 26 & 3.69 & 4.67 & .23 & .78 \\
\hline
\end{tabular}

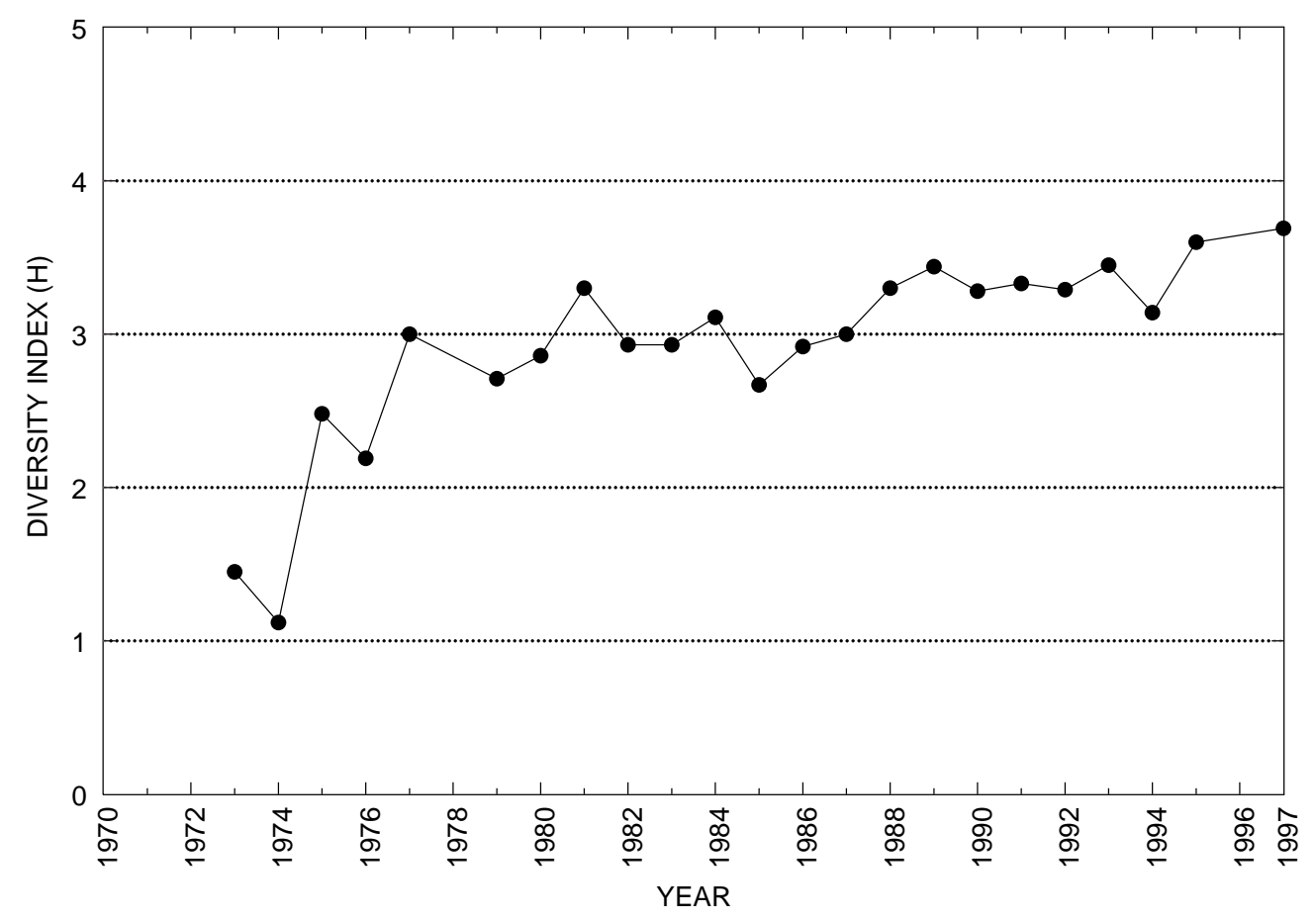


Table 6. Brillouin's diversity index, maximum diversity, minimum diversity, and relative evenness by site-Continued

01480640 (Site 38) - West Branch Brandywine Creek at Wawaset, Pa.

\begin{tabular}{ccccccc}
\hline Year & $\begin{array}{c}\text { Total } \\
\text { organisms }\end{array}$ & $\begin{array}{c}\text { Total } \\
\text { taxa }\end{array}$ & $\begin{array}{c}\text { Brillouin's } \\
\text { diversity } \\
\text { index }(\mathrm{H})\end{array}$ & $\begin{array}{c}\text { Maximum } \\
\text { diversity } \\
\left(\mathrm{H}_{\text {max }}\right)\end{array}$ & $\begin{array}{c}\text { Minimum } \\
\text { diversity } \\
\left(\mathrm{H}_{\text {min }}\right)\end{array}$ & $\begin{array}{c}\text { Evenness } \\
(\mathrm{E})\end{array}$ \\
\hline 1995 & 547 & 43 & 3.94 & 5.40 & 0.69 & 0.69 \\
1996 & 861 & 30 & 3.39 & 4.90 & .33 & .67 \\
1997 & 1,476 & 38 & 3.72 & 5.26 & .26 & .69 \\
\hline
\end{tabular}

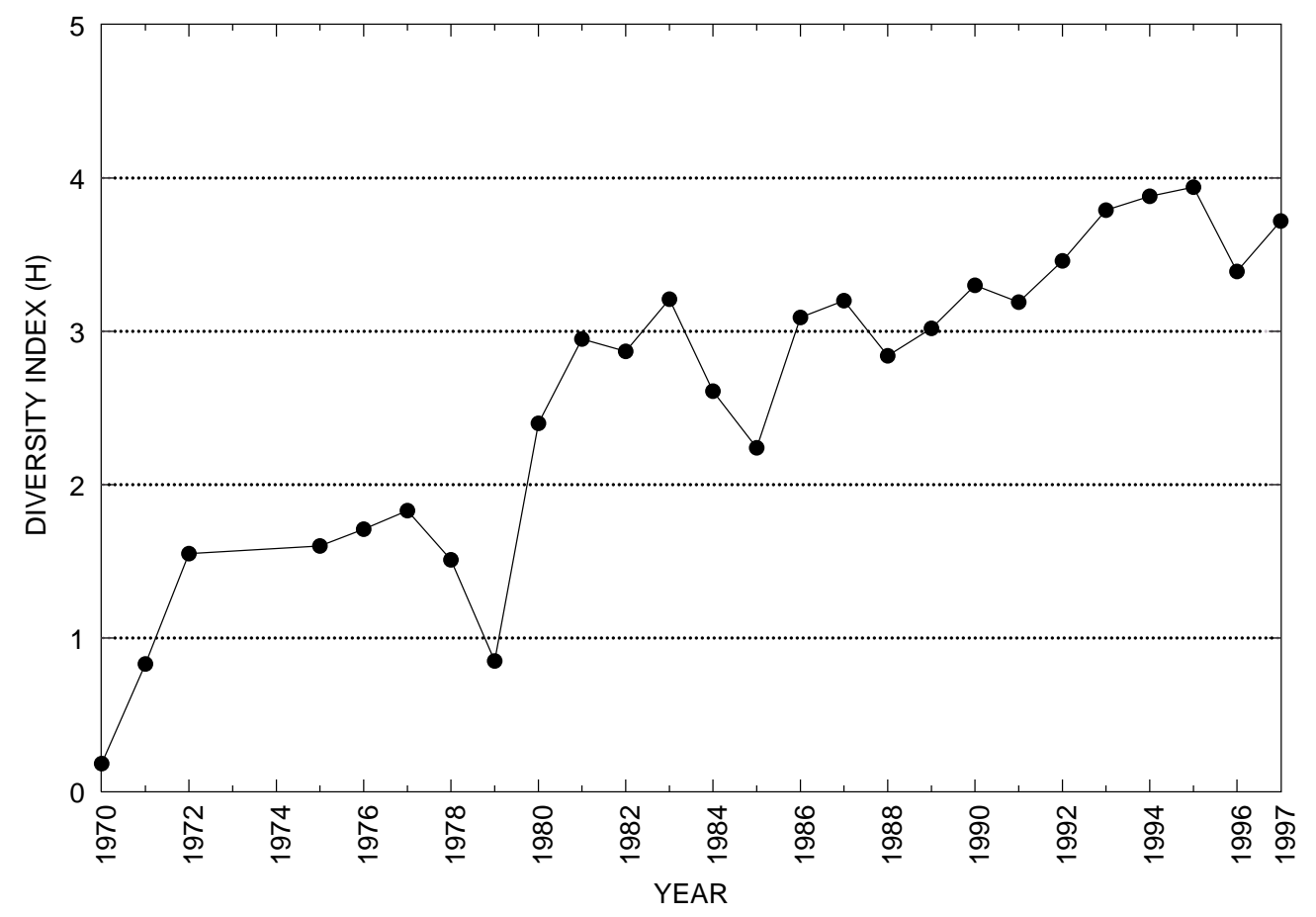


Table 6. Brillouin's diversity index, maximum diversity, minimum diversity, and relative evenness by site-Continued

[-, no data]

01480648 (Site 48) - East Branch Brandywine Creek near Cupola, Pa.

\begin{tabular}{ccccccc}
\hline Year & $\begin{array}{c}\text { Total } \\
\text { organisms }\end{array}$ & $\begin{array}{c}\text { Total } \\
\text { taxa }\end{array}$ & $\begin{array}{c}\text { Brillouin's } \\
\text { diversity } \\
\text { index }(\mathrm{H})\end{array}$ & $\begin{array}{c}\text { Maximum } \\
\text { diversity } \\
\left(\mathrm{H}_{\text {max }}\right)\end{array}$ & $\begin{array}{c}\text { Minimum } \\
\text { diversity } \\
\left(\mathrm{H}_{\text {min }}\right)\end{array}$ & $\begin{array}{c}\text { Evenness } \\
(\mathrm{E})\end{array}$ \\
\hline 1995 & 648 & 35 & 3.66 & 5.07 & 0.49 & 0.69 \\
1996 & - & - & - & - & - & - \\
1997 & - & - & - & - & - & - \\
\hline
\end{tabular}

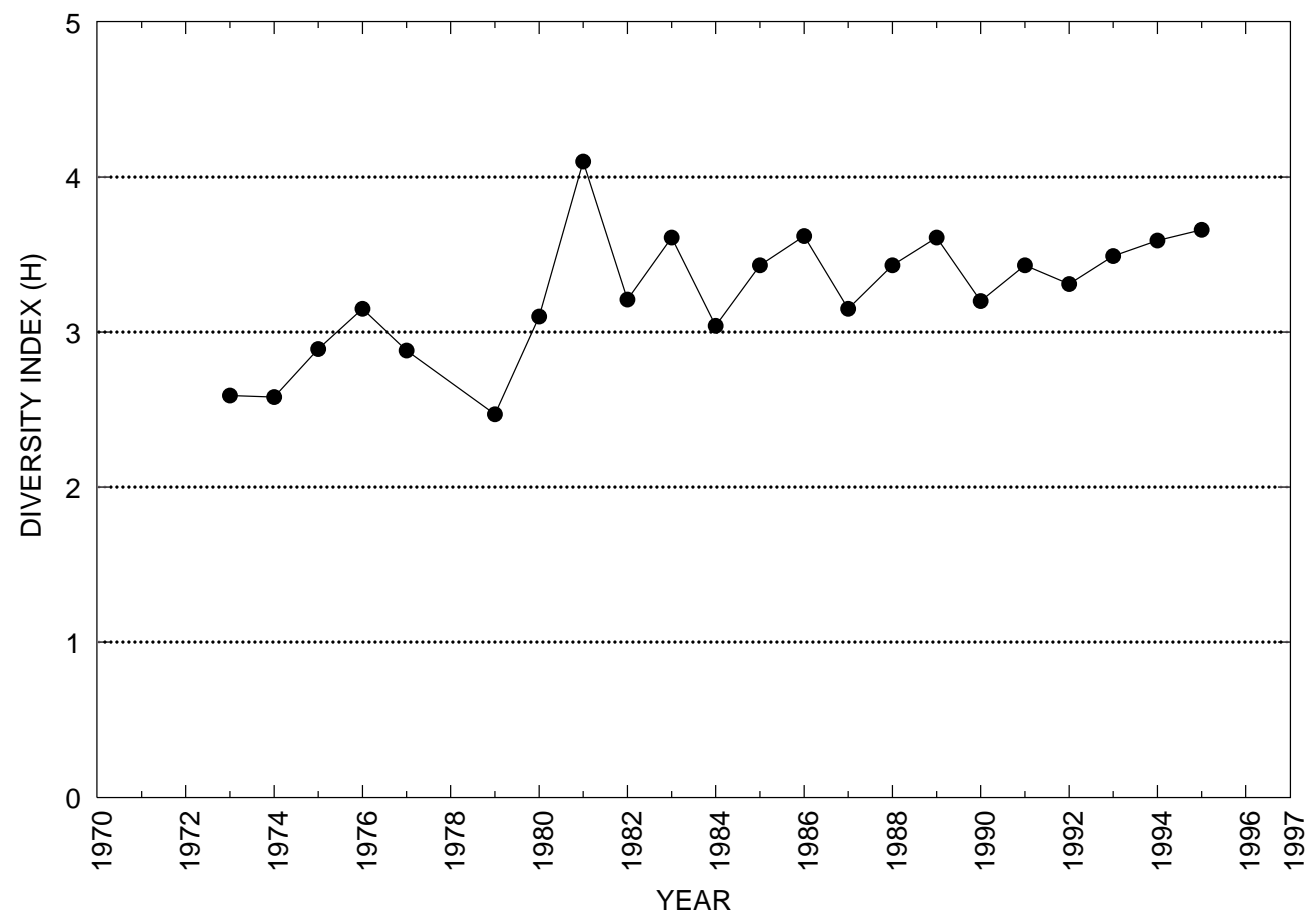


Table 6. Brillouin's diversity index, maximum diversity, minimum diversity, and relative evenness by site-Continued

[-, no data]

01480653 (Site 42) - East Branch Brandywine Creek at Glenmoore, Pa.

\begin{tabular}{ccccccc}
\hline Year & $\begin{array}{c}\text { Total } \\
\text { organisms }\end{array}$ & $\begin{array}{c}\text { Total } \\
\text { taxa }\end{array}$ & $\begin{array}{c}\text { Brillouin's } \\
\text { diversity } \\
\text { index }(\mathrm{H})\end{array}$ & $\begin{array}{c}\text { Maximum } \\
\text { diversity } \\
\left(\mathrm{H}_{\text {max }}\right)\end{array}$ & $\begin{array}{c}\text { Minimum } \\
\text { diversity } \\
\left(\mathrm{H}_{\text {min }}\right)\end{array}$ & $\begin{array}{c}\text { Evenness } \\
(\mathrm{E})\end{array}$ \\
\hline 1995 & 625 & 34 & 3.48 & 5.00 & 0.49 & 0.66 \\
1996 & - & - & - & - & - & - \\
1997 & - & - & - & - & - & - \\
\hline
\end{tabular}

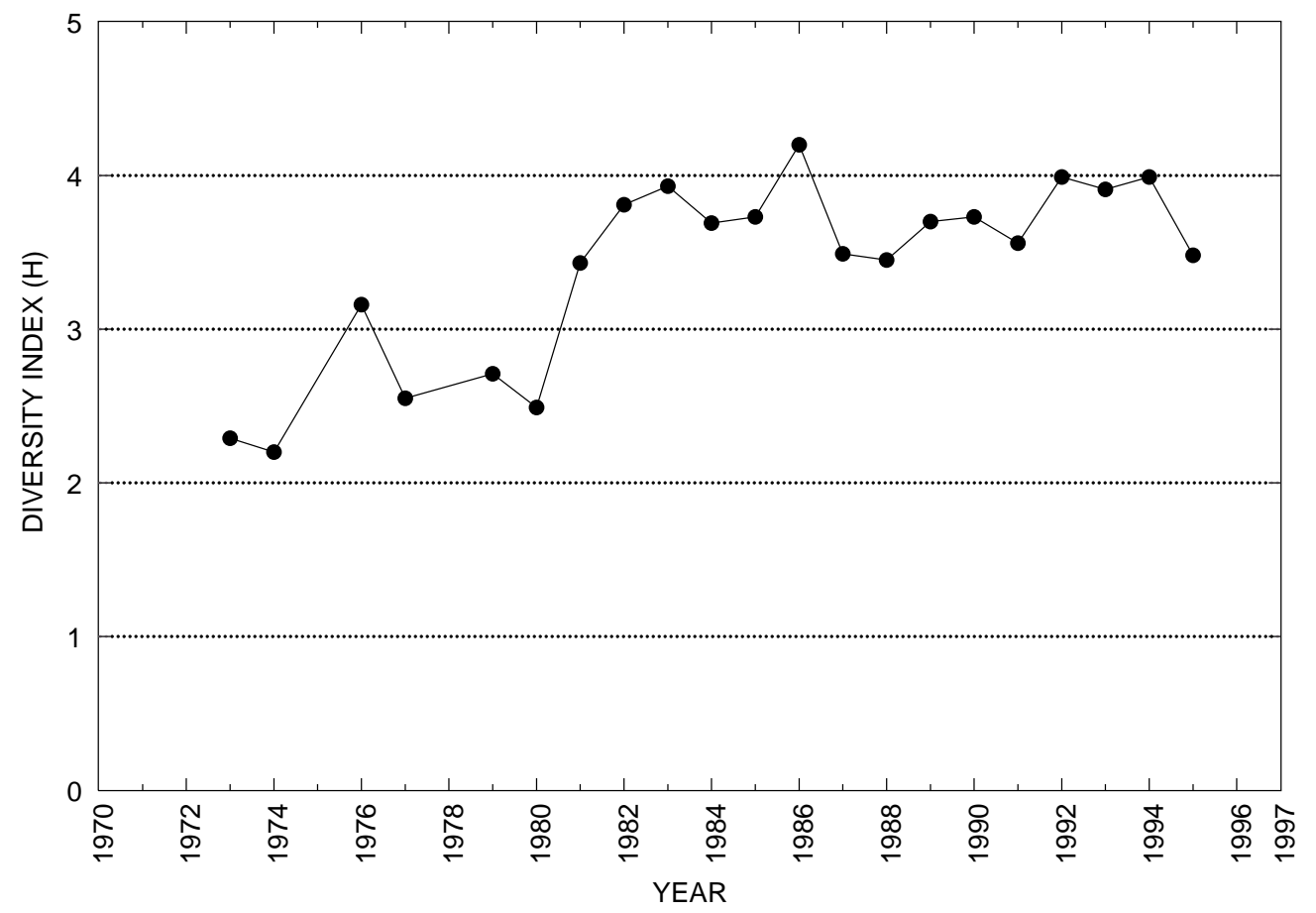


Table 6. Brillouin's diversity index, maximum diversity, minimum diversity, and relative evenness by site-Continued

[-, no data]

01480656 (Site 47) - Indian Run near Springton, Pa.

\begin{tabular}{ccccccc}
\hline Year & $\begin{array}{c}\text { Total } \\
\text { organisms }\end{array}$ & $\begin{array}{c}\text { Total } \\
\text { taxa }\end{array}$ & $\begin{array}{c}\text { Brillouin's } \\
\text { diversity } \\
\text { index }(\mathrm{H})\end{array}$ & $\begin{array}{c}\text { Maximum } \\
\text { diversity } \\
\left(\mathrm{H}_{\text {max }}\right)\end{array}$ & $\begin{array}{c}\text { Minimum } \\
\text { diversity } \\
\left(\mathrm{H}_{\text {min }}\right)\end{array}$ & $\begin{array}{c}\text { Evenness } \\
(\mathrm{E})\end{array}$ \\
\hline 1995 & 647 & 33 & 3.37 & 5.01 & 0.46 & 0.64 \\
1996 & - & - & - & - & - & - \\
1997 & - & - & - & - & - & - \\
\hline
\end{tabular}

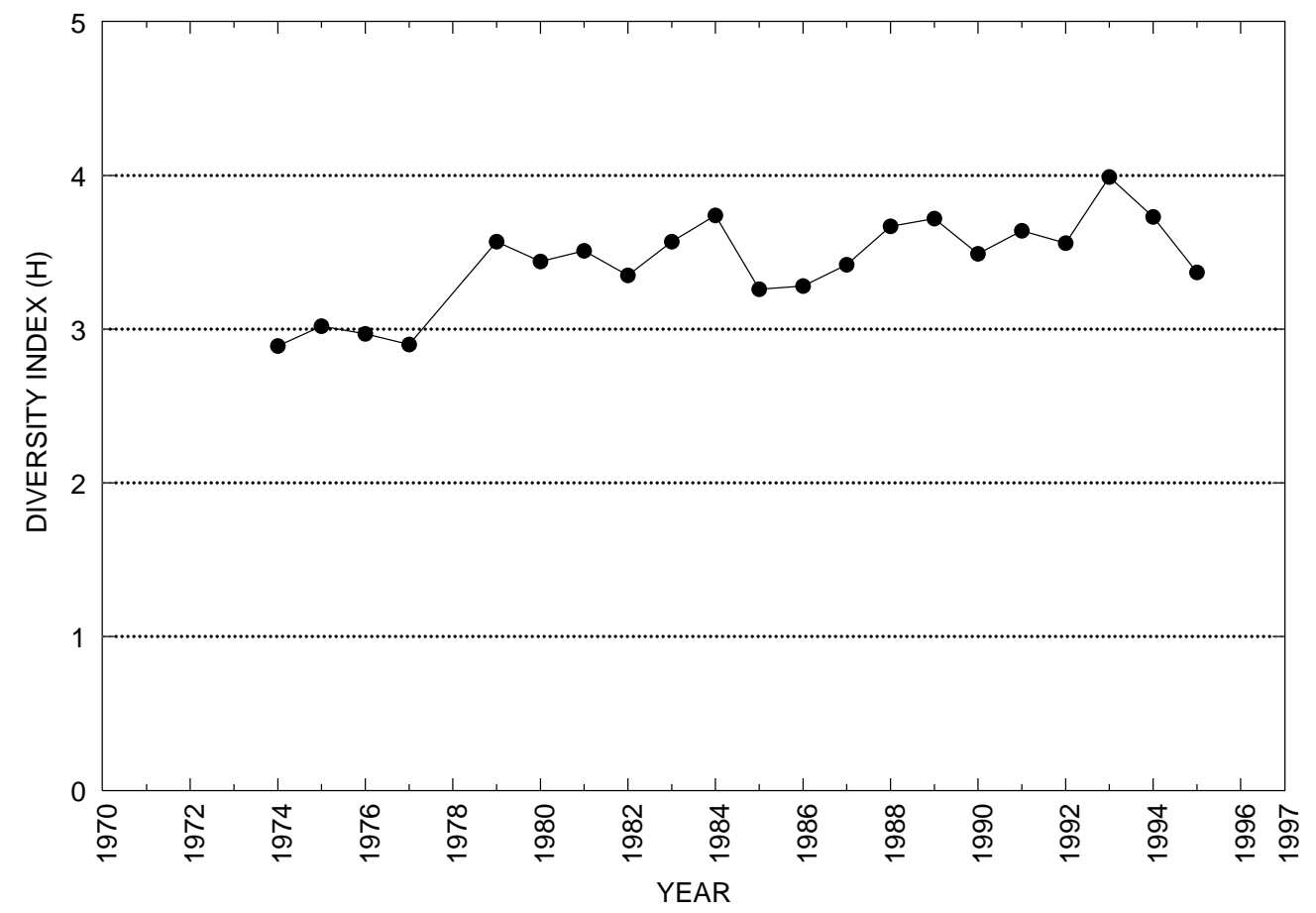


Table 6. Brillouin's diversity index, maximum diversity, minimum diversity, and relative evenness by site-Continued

[-, no data]

01480700 (Site 36) - East Branch Brandywine Creek near Downingtown, Pa.

\begin{tabular}{ccccccc}
\hline Year & $\begin{array}{c}\text { Total } \\
\text { organisms }\end{array}$ & $\begin{array}{c}\text { Total } \\
\text { taxa }\end{array}$ & $\begin{array}{c}\text { Brillouin's } \\
\text { diversity } \\
\text { index }(\mathrm{H})\end{array}$ & $\begin{array}{c}\text { Maximum } \\
\text { diversity } \\
\left(\mathrm{H}_{\text {max }}\right)\end{array}$ & $\begin{array}{c}\text { Minimum } \\
\text { diversity } \\
\left(\mathrm{H}_{\text {min }}\right)\end{array}$ & $\begin{array}{c}\text { Evenness } \\
(\mathrm{E})\end{array}$ \\
\hline 1995 & 1,508 & 41 & 3.53 & 5.37 & 0.28 & 0.64 \\
1996 & 1,347 & 49 & 4.01 & 5.57 & .37 & .70 \\
1997 & - & - & - & - & - & - \\
\hline
\end{tabular}

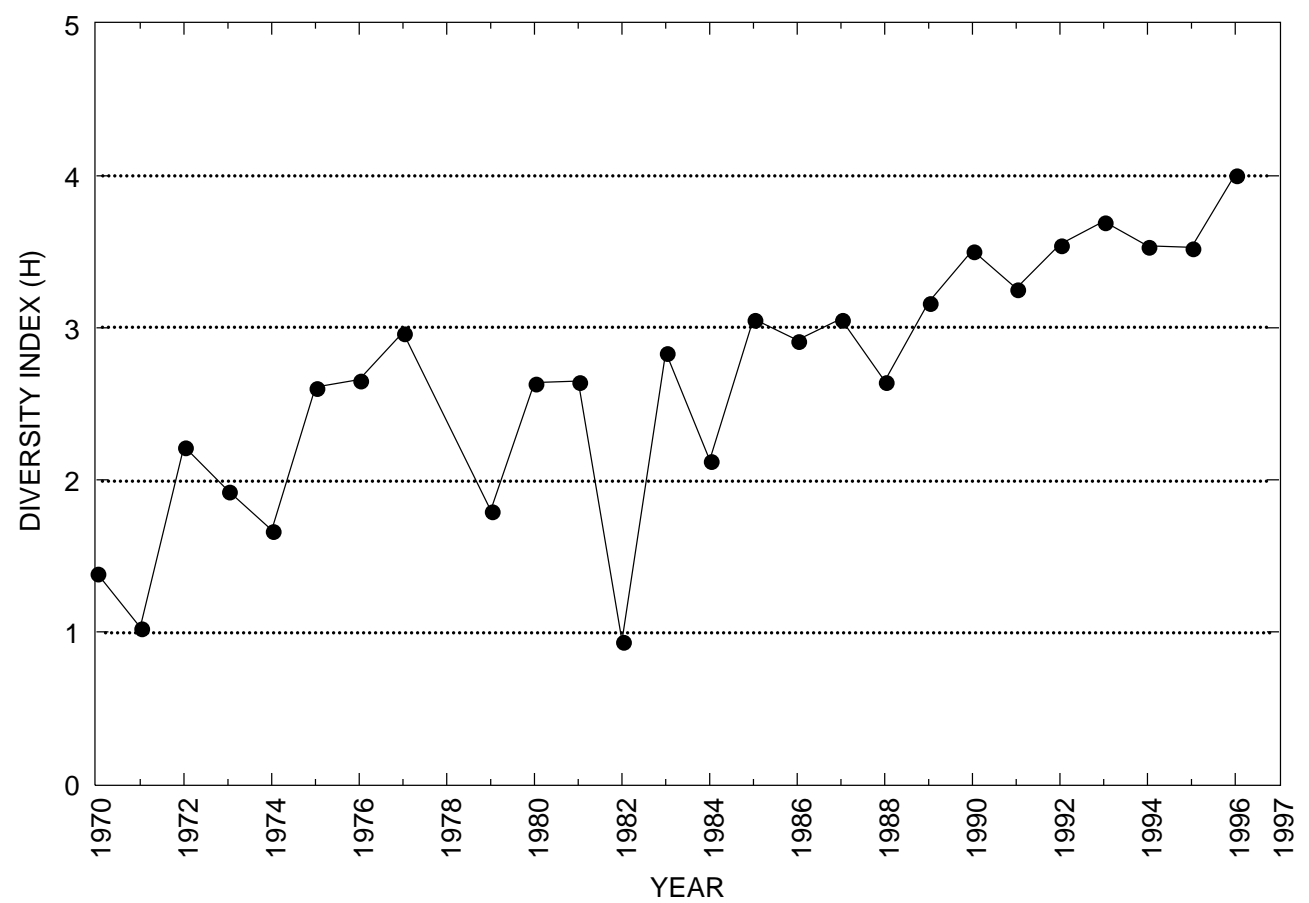


Table 6. Brillouin's diversity index, maximum diversity, minimum diversity, and relative evenness by site-Continued

[-, no data]

01480903 (Site 44) - Valley Creek at Mullsteins Meadows near Downingtown, Pa.

\begin{tabular}{ccccccc}
\hline Year & $\begin{array}{c}\text { Total } \\
\text { organisms }\end{array}$ & $\begin{array}{c}\text { Total } \\
\text { taxa }\end{array}$ & $\begin{array}{c}\text { Brillouin's } \\
\text { diversity } \\
\text { index }(\mathrm{H})\end{array}$ & $\begin{array}{c}\text { Maximum } \\
\text { diversity } \\
\left(\mathrm{H}_{\text {max }}\right)\end{array}$ & $\begin{array}{c}\text { Minimum } \\
\text { diversity } \\
\left(\mathrm{H}_{\text {min }}\right)\end{array}$ & $\begin{array}{c}\text { Evenness } \\
(\mathrm{E})\end{array}$ \\
\hline 1995 & 1,327 & 34 & 3.50 & 4.99 & 0.26 & 0.69 \\
1996 & - & - & - & - & - & - \\
1997 & 3,137 & 36 & 3.30 & 5.13 & .13 & .63 \\
\hline
\end{tabular}

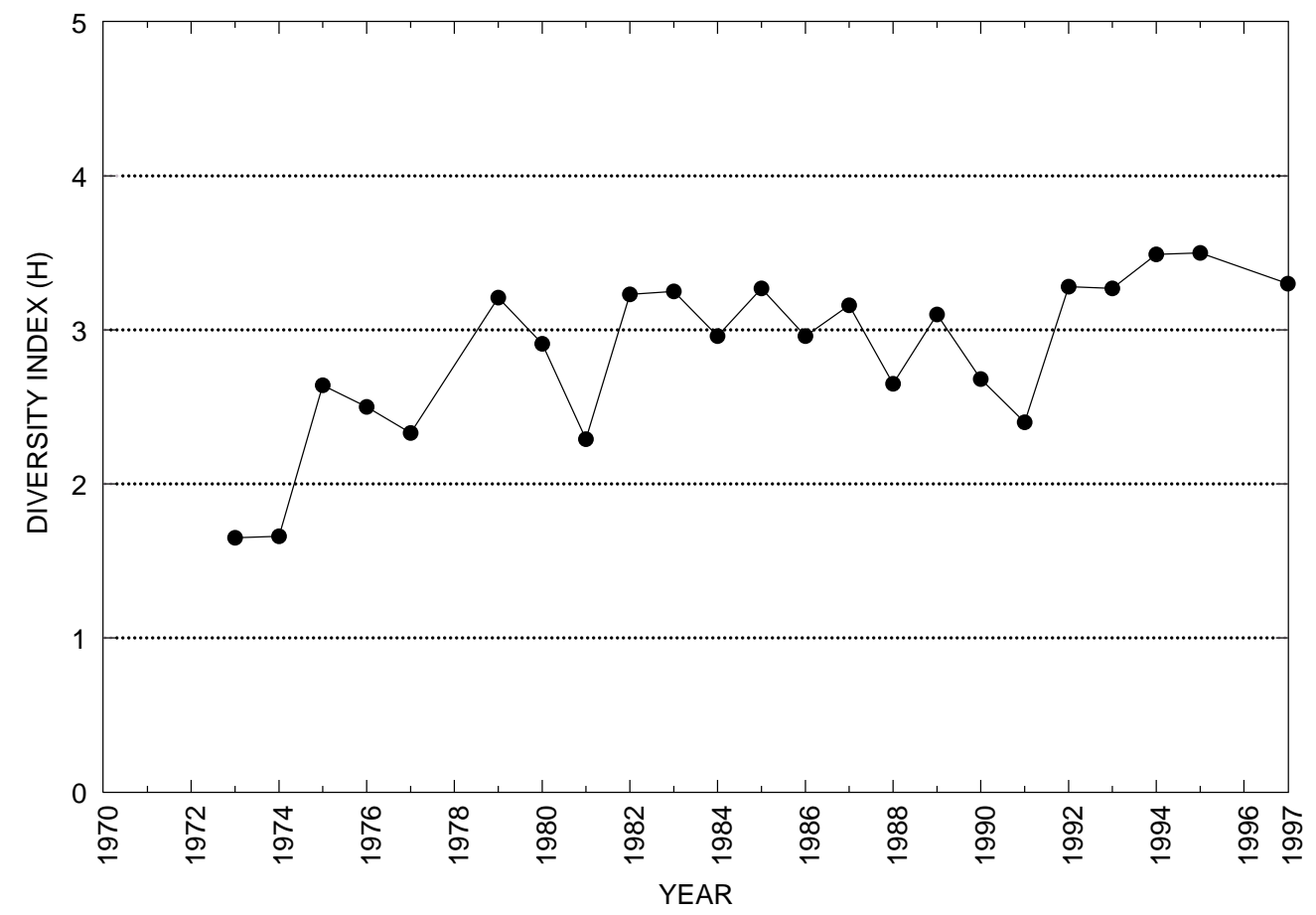


Table 6. Brillouin's diversity index, maximum diversity, minimum diversity, and relative evenness by site-Continued

01480950 (Site 39) - East Branch Brandywine Creek at Wawaset, Pa.

\begin{tabular}{ccccccc}
\hline Year & $\begin{array}{c}\text { Total } \\
\text { organisms }\end{array}$ & $\begin{array}{c}\text { Total } \\
\text { taxa }\end{array}$ & $\begin{array}{c}\text { Brillouin's } \\
\text { diversity } \\
\text { index }(\mathrm{H})\end{array}$ & $\begin{array}{c}\text { Maximum } \\
\text { diversity } \\
\left(\mathrm{H}_{\text {max }}\right)\end{array}$ & $\begin{array}{c}\text { Minimum } \\
\text { diversity } \\
\left(\mathrm{H}_{\text {min }}\right)\end{array}$ & $\begin{array}{c}\text { Evenness } \\
(\mathrm{E})\end{array}$ \\
\hline 1995 & 307 & 33 & 3.59 & 4.85 & 0.85 & 0.68 \\
1996 & 880 & 32 & 3.30 & 4.96 & .34 & .64 \\
1997 & 2,123 & 30 & 3.21 & 4.91 & .15 & .64 \\
\hline
\end{tabular}

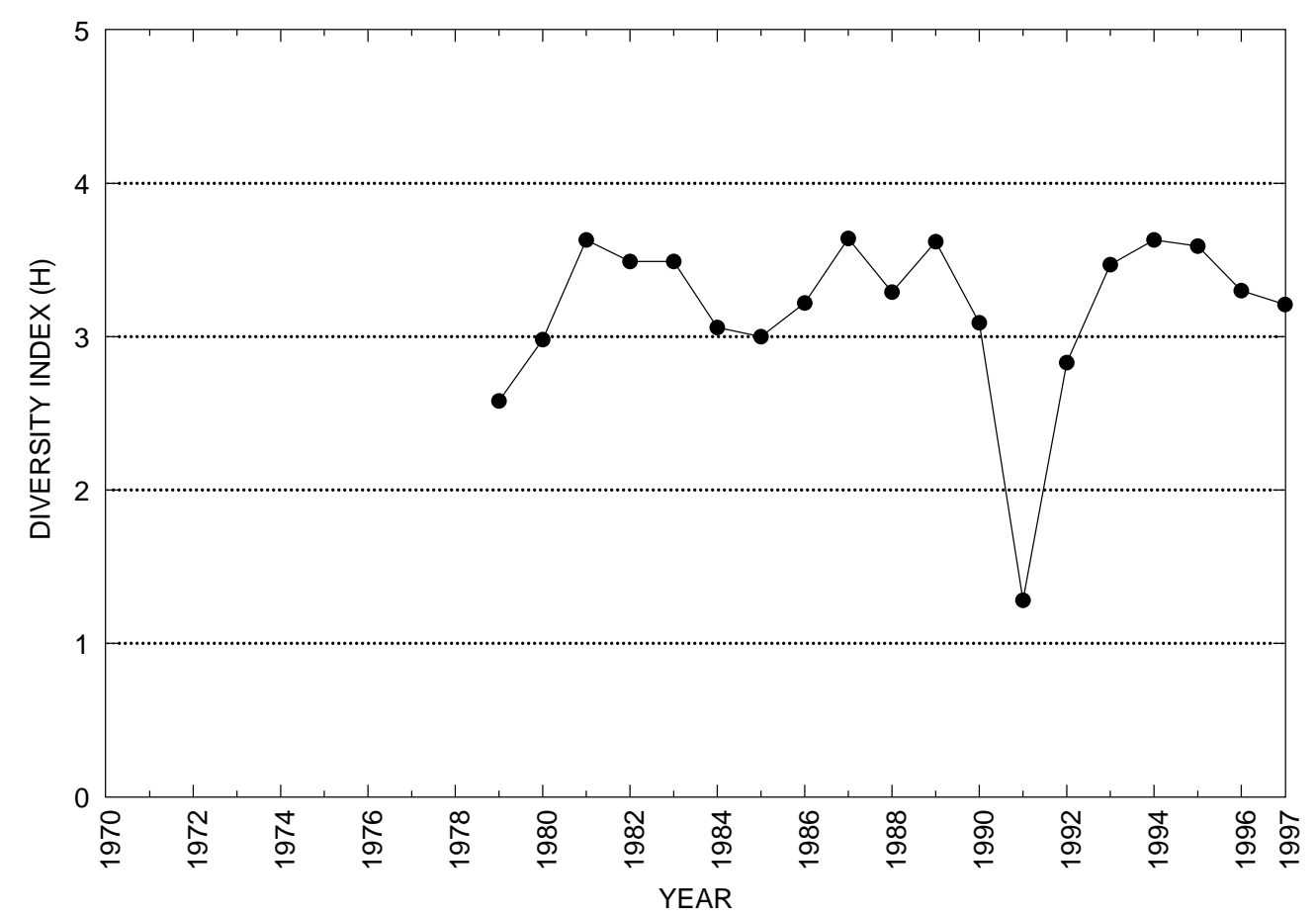


Table 6. Brillouin's diversity index, maximum diversity, minimum diversity, and relative evenness by site-Continued

[-, no data]

01481030 (Site 40) - Brandywine Creek near Chadds Ford, Pa.

\begin{tabular}{ccccccc}
\hline Year & $\begin{array}{c}\text { Total } \\
\text { organisms }\end{array}$ & $\begin{array}{c}\text { Total } \\
\text { taxa }\end{array}$ & $\begin{array}{c}\text { Brillouin's } \\
\text { diversity } \\
\text { index }(\mathrm{H})\end{array}$ & $\begin{array}{c}\text { Maximum } \\
\text { diversity } \\
\left(\mathrm{H}_{\text {max }}\right)\end{array}$ & $\begin{array}{c}\text { Minimum } \\
\text { diversity } \\
\left(\mathrm{H}_{\text {min }}\right)\end{array}$ & $\begin{array}{c}\text { Evenness } \\
(\mathrm{E})\end{array}$ \\
\hline 1995 & 1,208 & 33 & 3.77 & 5.03 & 0.27 & 0.74 \\
1996 & - & - & - & - & - & - \\
1997 & 3,000 & 41 & 3.37 & 5.37 & .16 & .62 \\
\hline
\end{tabular}

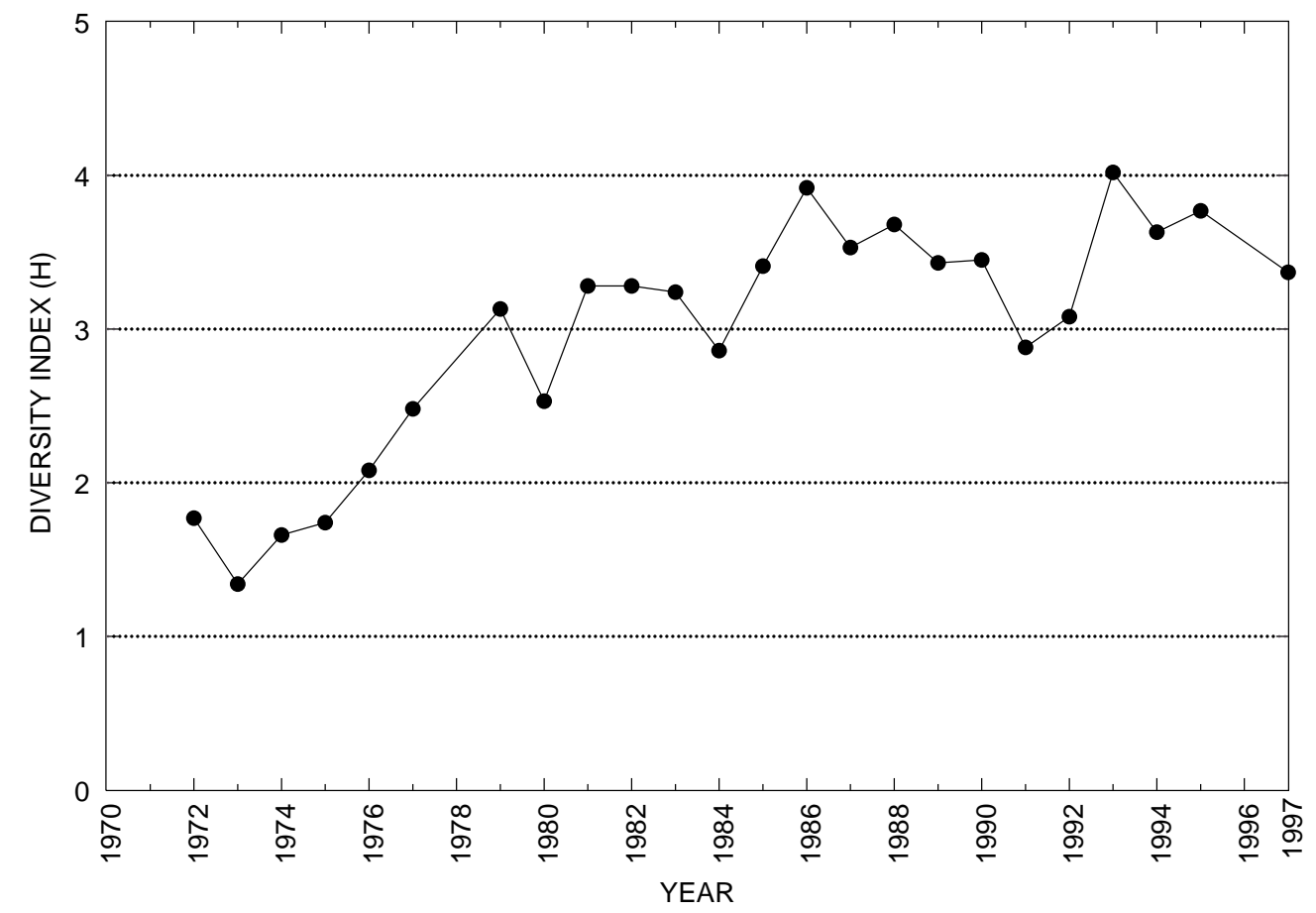


Table 6. Brillouin's diversity index, maximum diversity, minimum diversity, and relative evenness by site-Continued

01494900 (Site 31) - East Branch Big Elk Creek at Elkview, Pa.

\begin{tabular}{ccccccc}
\hline Year & $\begin{array}{c}\text { Total } \\
\text { organisms }\end{array}$ & $\begin{array}{c}\text { Total } \\
\text { taxa }\end{array}$ & $\begin{array}{c}\text { Brillouin's } \\
\text { diversity } \\
\text { index }(\mathrm{H})\end{array}$ & $\begin{array}{c}\text { Maximum } \\
\text { diversity } \\
\left(\mathrm{H}_{\text {max }}\right)\end{array}$ & $\begin{array}{c}\text { Minimum } \\
\text { diversity } \\
\left(\mathrm{H}_{\text {min }}\right)\end{array}$ & $\begin{array}{c}\text { Evenness } \\
(\mathrm{E})\end{array}$ \\
\hline 1995 & 124 & 14 & 2.45 & 3.82 & 0.72 & 0.56 \\
1996 & 71 & 11 & 2.29 & 3.30 & .85 & .59 \\
1997 & 529 & 13 & 1.39 & 3.70 & .20 & .34 \\
\hline
\end{tabular}

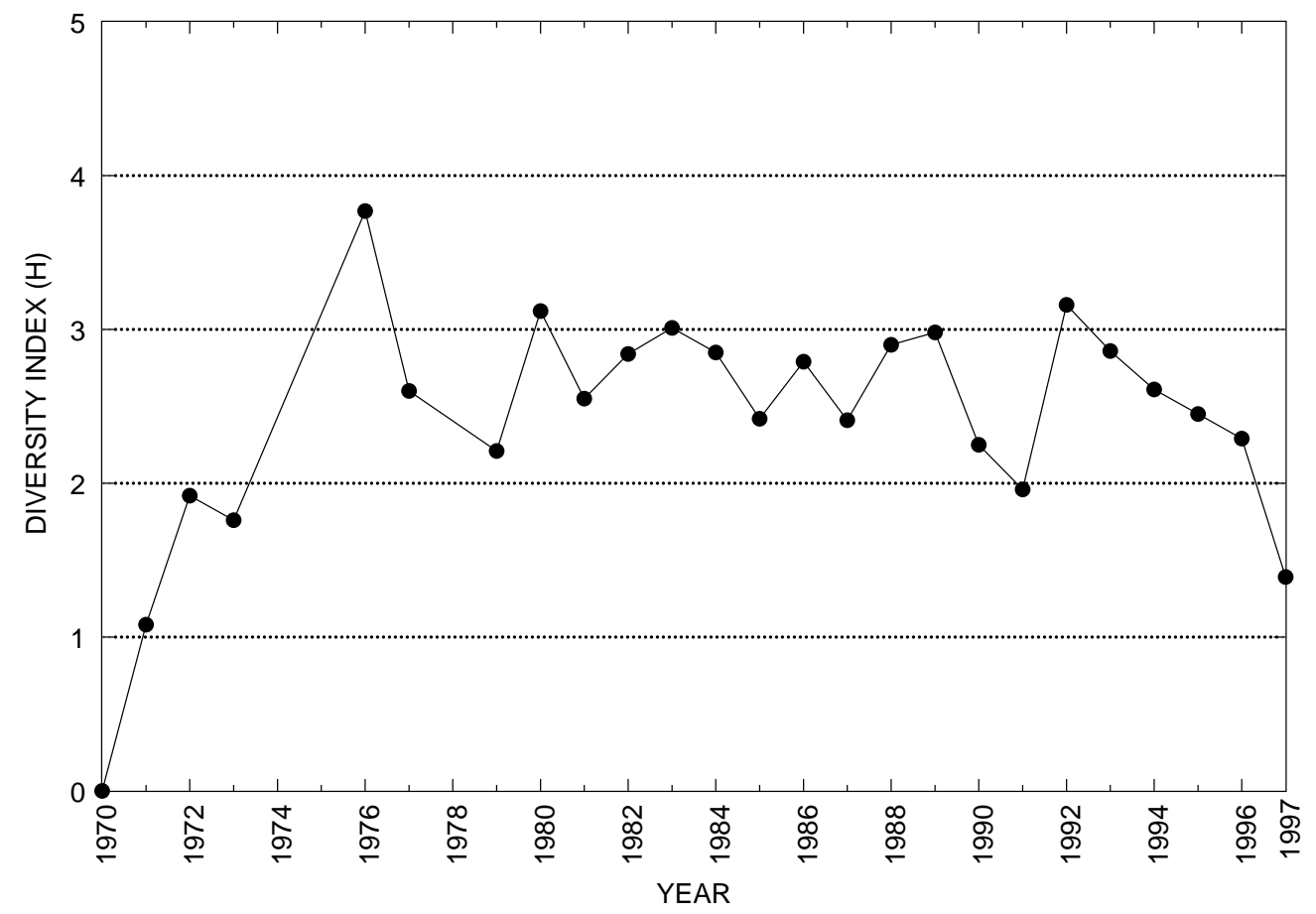


Table 6. Brillouin's diversity index, maximum diversity, minimum diversity, and relative evenness by site-Continued

[-, no data]

01494950 (Site 32) - West Branch Big Elk Creek near Oxford, Pa.

\begin{tabular}{ccccccc}
\hline Year & $\begin{array}{c}\text { Total } \\
\text { organisms }\end{array}$ & $\begin{array}{c}\text { Total } \\
\text { taxa }\end{array}$ & $\begin{array}{c}\text { Brillouin's } \\
\text { diversity } \\
\text { index }(\mathrm{H})\end{array}$ & $\begin{array}{c}\text { Maximum } \\
\text { diversity } \\
\left(\mathrm{H}_{\text {max }}\right)\end{array}$ & $\begin{array}{c}\text { Minimum } \\
\text { diversity } \\
\left(\mathrm{H}_{\text {min }}\right)\end{array}$ & $\begin{array}{c}\text { Evenness } \\
(\mathrm{E})\end{array}$ \\
\hline 1995 & 85 & 15 & 2.06 & 3.80 & 0.32 & 0.37 \\
1996 & 290 & 20 & 2.97 & 4.25 & .53 & .66 \\
1997 & - & - & - & - & - & - \\
\hline
\end{tabular}

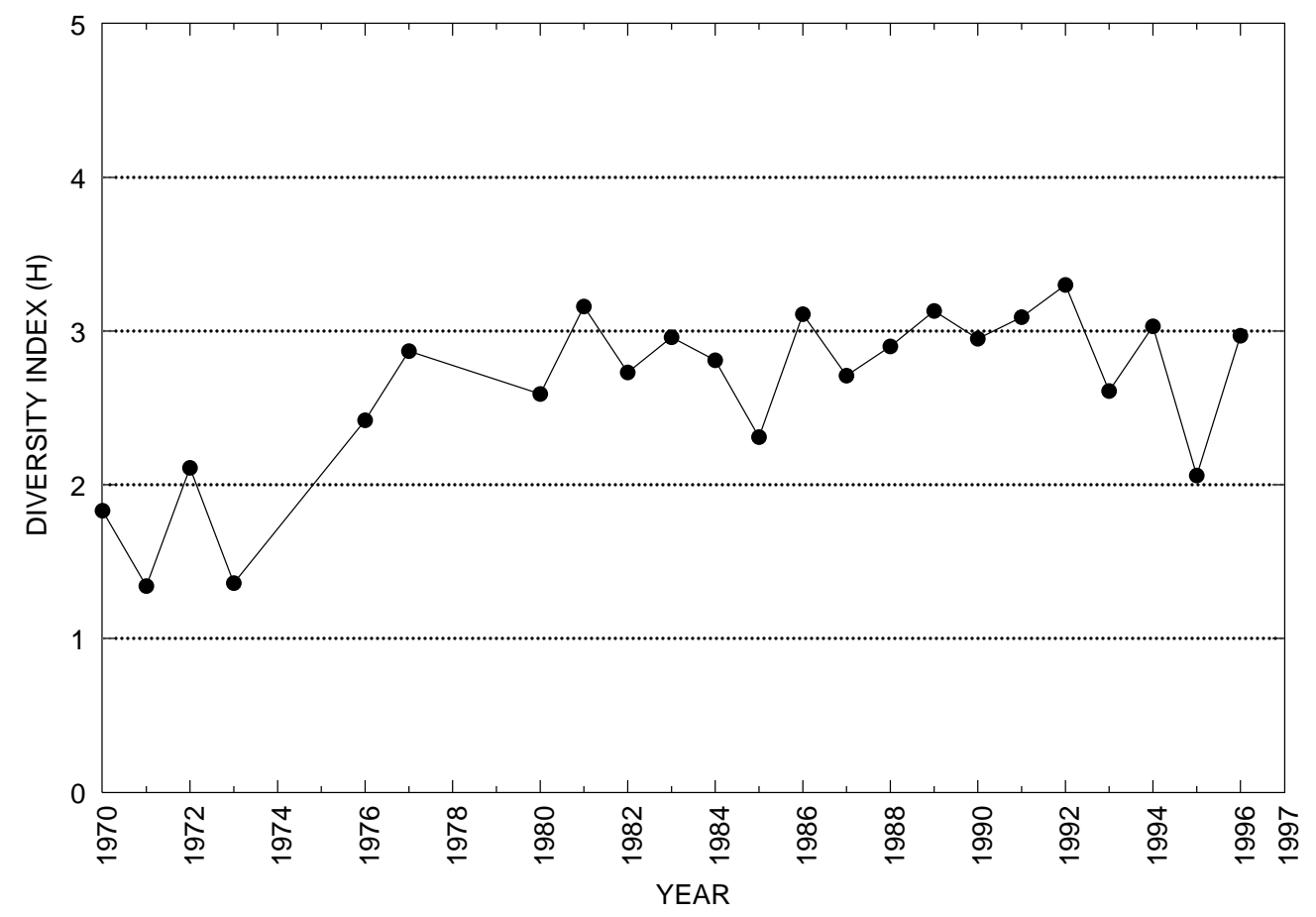


Table 6. Brillouin's diversity index, maximum diversity, minimum diversity, and relative evenness by site-Continued

01578340 (Site 33) - East Branch Octoraro Creek at Christiana, Pa.

\begin{tabular}{ccccccc}
\hline Year & $\begin{array}{c}\text { Total } \\
\text { organisms }\end{array}$ & $\begin{array}{c}\text { Total } \\
\text { taxa }\end{array}$ & $\begin{array}{c}\text { Brillouin's } \\
\text { diversity } \\
\text { index }(\mathrm{H})\end{array}$ & $\begin{array}{c}\text { Maximum } \\
\text { diversity } \\
\left(\mathrm{H}_{\text {max }}\right)\end{array}$ & $\begin{array}{c}\text { Minimum } \\
\text { diversity } \\
\left(\mathrm{H}_{\text {min }}\right)\end{array}$ & $\begin{array}{c}\text { Evenness } \\
(\mathrm{E})\end{array}$ \\
\hline 1995 & 719 & 26 & 3.05 & 4.69 & 0.33 & 0.62 \\
1996 & 847 & 25 & 3.36 & 4.67 & .28 & .70 \\
1997 & 1,478 & 29 & 3.08 & 4.88 & .20 & .61 \\
\hline
\end{tabular}

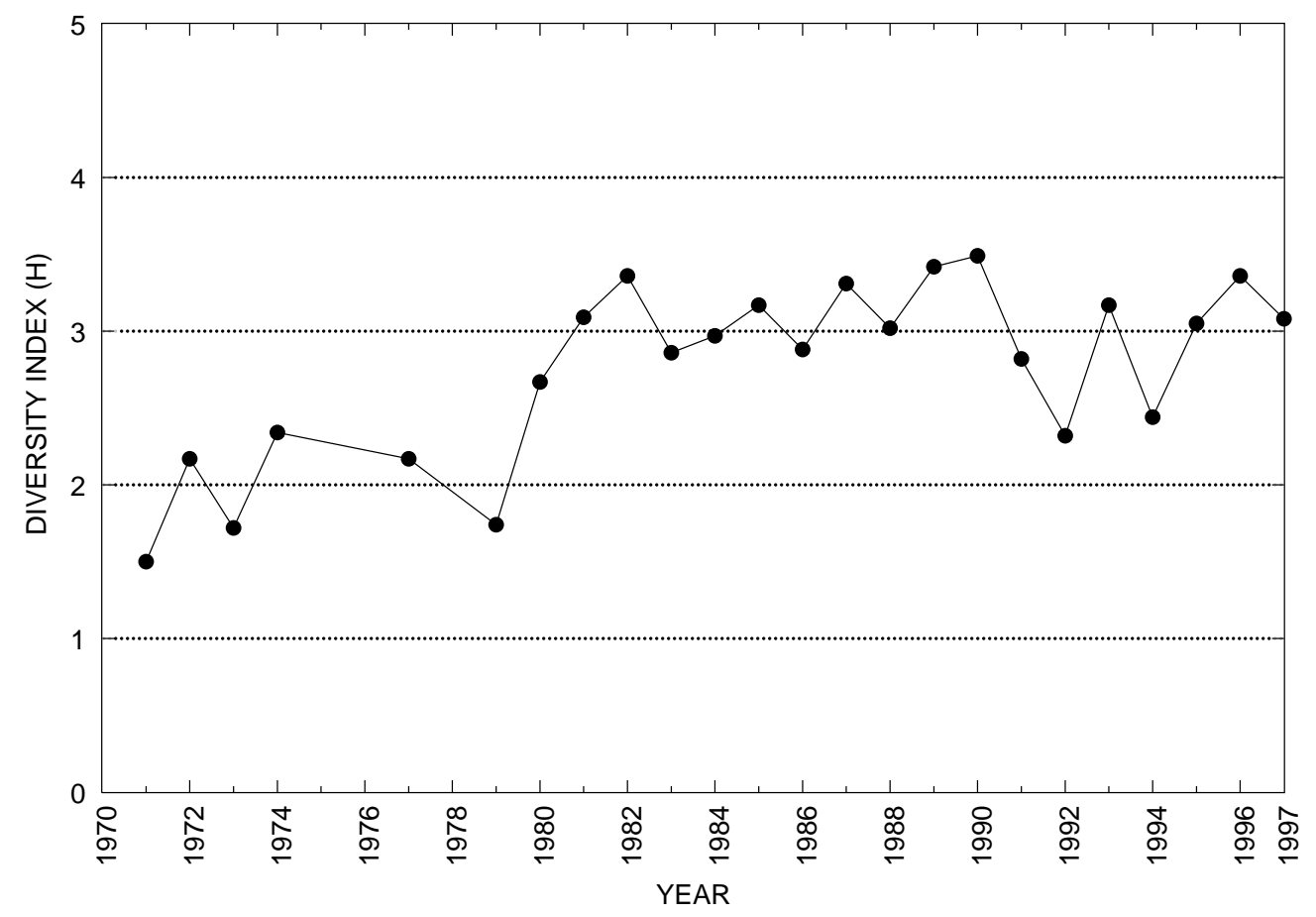


Table 6. Brillouin's diversity index, maximum diversity, minimum diversity, and relative evenness by site-Continued

[-, no data]

01578343 (Site 34) - Valley Creek near Atglen, Pa.

\begin{tabular}{ccccccc}
\hline Year & $\begin{array}{c}\text { Total } \\
\text { organisms }\end{array}$ & $\begin{array}{c}\text { Total } \\
\text { taxa }\end{array}$ & $\begin{array}{c}\text { Brillouin's } \\
\text { diversity } \\
\text { index }(\mathrm{H})\end{array}$ & $\begin{array}{c}\text { Maximum } \\
\text { diversity } \\
\left(\mathrm{H}_{\text {max }}\right)\end{array}$ & $\begin{array}{c}\text { Minimum } \\
\text { diversity } \\
\left(\mathrm{H}_{\text {min }}\right)\end{array}$ & $\begin{array}{c}\text { Evenness } \\
(\mathrm{E})\end{array}$ \\
\hline 1995 & 460 & 21 & 2.84 & 4.43 & 0.38 & 0.61 \\
1996 & 1,855 & 31 & 3.20 & 4.97 & .18 & .63 \\
1997 & - & - & - & - & - & - \\
\hline
\end{tabular}

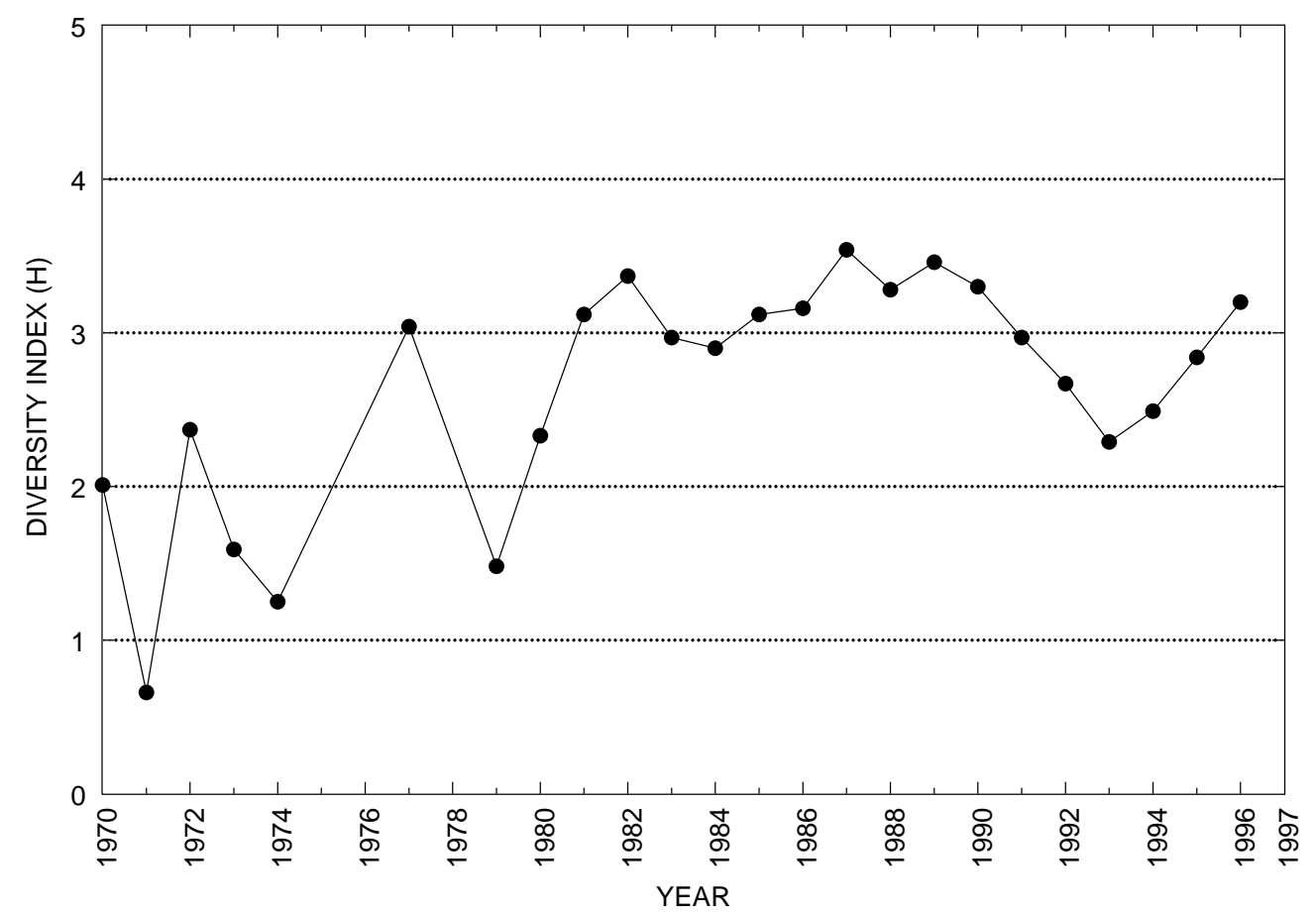


Table 7. Alphabetical checklist of taxa reported from Chester County Biological Monitoring Network, 1995-97

\begin{tabular}{|c|c|c|}
\hline Acroneuria & Ferrissia & Paragnetina \\
\hline Agnetina & & Paraleptophlebia \\
\hline Allocapnia & Gammarus & Paraleuctra \\
\hline Amnicola & $\begin{array}{l}\text { Glossosoma } \\
\text { Geera }\end{array}$ & Paranemoura \\
\hline Ancyronyx & Goera & Parapsyche \\
\hline Anthopotamus & Gomphidae & Perlidae \\
\hline Antocha & Gomphus & Petrophila \\
\hline Apatania & Gyraulus & Physa \\
\hline Archanara & Helicopsyche & Pisidium \\
\hline Argia & Helobdella & Planariidae \\
\hline Asellidae & Helophorus & Podocopa \\
\hline Atherix & Hemerodromia & Polycentropus \\
\hline & Hexagenia & Promoresia \\
\hline $\begin{array}{l}\text { Baetıs } \\
\text { Berosus }\end{array}$ & Hexatoma & Prosimulium \\
\hline Berosus & Hydatophylax & Prostoma \\
\hline Brachycentrus & Hydrachnidia & Protoptila \\
\hline Caecidotea & Hydropsyche & Psephenus \\
\hline Caenis & Hydroptila & Pseudocloeon \\
\hline Cambaridae & Iconushia & Psilotreta \\
\hline Capniidae & Isonychıa & Psychomyia \\
\hline Ceratopogonidae & Lanthus & \\
\hline Ceratopsyche & Lepidostoma & $\begin{array}{l}\text { Rhagovelia } \\
\text { Rhyacophila }\end{array}$ \\
\hline Chelifera & Leucotrichia & Rhyacophila \\
\hline Cheumatopsyche & Limnophila & Sialis \\
\hline Chimarra & Lirceus & Sigara \\
\hline Chironomidae & Lumbriculida & Simulium \\
\hline Chloroperlidae & Lymnaea & Sphaeriidae \\
\hline Chrysomelidae & Macronuchus & Stenacron \\
\hline Cladocera & Macronychus & Stenelmis \\
\hline Clinocera & Macrostemum & Stenonema \\
\hline Coenagrionidae & $\begin{array}{l}\text { Manayunkıa speciosa } \\
\text { Metrobates }\end{array}$ & Strophopteryx \\
\hline Corixidae & Micrasema & Taeniopteryx \\
\hline Corydalus & Microcylloepus & Tipula \\
\hline Crangonyx & Musculium & Tricorythodes \\
\hline $\begin{array}{l}\text { Curculionidae } \\
\text { Cyclopidae }\end{array}$ & Mystacides & Tubificidae \\
\hline Cyrnellus & Naididae & Wormaldia \\
\hline Dicranata & Nematoda & \\
\hline $\begin{array}{l}\text { Dicranota } \\
\text { Dinlectrong }\end{array}$ & Nemouridae & \\
\hline Diplectrona & Neophylax & \\
\hline $\begin{array}{l}\text { Dolophilodes } \\
\text { Dubiraphia }\end{array}$ & Neureclipsis & \\
\hline Dubiraphia & Nigronia & \\
\hline Ectopria & Nyctiophylax & \\
\hline Empididae & Oecetis & \\
\hline $\begin{array}{l}\text { Epeorus } \\
\text { Fnhemera }\end{array}$ & Oligochaeta & \\
\hline $\begin{array}{l}\text { Ephemera } \\
\text { Fnhemerella }\end{array}$ & Optioservus & \\
\hline $\begin{array}{l}\text { Ephemerella } \\
\text { Ephydridae }\end{array}$ & Oulimnius & \\
\hline $\begin{array}{l}\text { Ephydridae } \\
\text { Erpobdellidae }\end{array}$ & & \\
\hline
\end{tabular}


Table 8. Systematic checklist of taxa reported from Chester County Biological Monitoring Network, 1995-97

\begin{tabular}{|c|c|}
\hline Platyhelminthes & Isopoda (SOW BUGS) \\
\hline Turbellaria (FLATWORMS) & Asellidae \\
\hline Tricladida & Caecidotea \\
\hline Planariidae & Lirceus \\
\hline Nematoda (NEMATODES) & Decapoda \\
\hline Nemertea (PROBOSCIS WORMS) & Cambaridae (CRAYFISH) \\
\hline Enopla & Podocopa (SEED SHRIMP) \\
\hline Hoplonemertea & Insecta \\
\hline Tetrastemmatidae & Ephemeroptera (MAYFLIES) \\
\hline Prostoma & Baetidae \\
\hline Mollusca & Baetis \\
\hline Gastropoda (SNAILS) & Pseudocloeon \\
\hline Mesogastropoda & Caenidae \\
\hline Hydrobiidae & Caenis \\
\hline Amnicola & Ephemerellidae \\
\hline Basommatophora & Ephemerella \\
\hline Ancylidae & Ephemeridae \\
\hline Ferrissia & Ephemera \\
\hline Gyraulus & Heptageniidae \\
\hline Lymnaeidae & Epeorus \\
\hline Lymnaea & Stenacron \\
\hline Physidae & Stenonema \\
\hline Physa & Isonychiidae \\
\hline Bivalvia (CLAMS) & Isonychia \\
\hline Veneroida & Leptophlebiidae \\
\hline Sphaeriidae & Paraleptophlebia \\
\hline Musculium & Potamanthidae \\
\hline Pisidium & Anthopotamus \\
\hline Annelida (SEGMENTED WORMS) & Tricorythidae \\
\hline Oligochaeta & Tricorythodes \\
\hline Lumbriculida & Odonata (DRAGONFLIES AND DAMSELFLIES) \\
\hline Lumbriculidae & Calopterygidae \\
\hline Tubificida & Hetaerina \\
\hline Naididae & Coenagrionidae \\
\hline Polychaeta & Argia \\
\hline Sabellida & Gomphidae \\
\hline Sabellidae & Gomphus \\
\hline Manayunkia speciosa & Lanthus \\
\hline Hirudinea (LEECHES) & Plecoptera (STONEFLIES) \\
\hline Pharynobdellidae & Capniidae \\
\hline Erpobdellidae & Allocapnia \\
\hline Rhynchobdellida & Chloroperlidae \\
\hline Glossiphoniidae & Leuctridae \\
\hline Helobdella & Paraleuctra \\
\hline Arthropoda & Nemouridae \\
\hline Acariformes & Paranemoura \\
\hline Hydrachnidia (WATER MITES) & Perlidae \\
\hline Crustacea & Acroneuria \\
\hline Cladocera & Agnetina \\
\hline Cyclopoida & Paragnetina \\
\hline Cyclopidae & Taeniopterygidae \\
\hline Amphipoda (SCUDS) & Strophopteryx \\
\hline Crangonyctidae & Taeniopteryx \\
\hline Crangonyx & Hemiptera (TRUE BUGS) \\
\hline Gammaridae & Corixidae \\
\hline Gammarus & Sigara \\
\hline & Gerridae \\
\hline
\end{tabular}


Table 8. Systematic checklist of taxa reported from Chester County Biological Monitoring Network, 1995-97-Continued

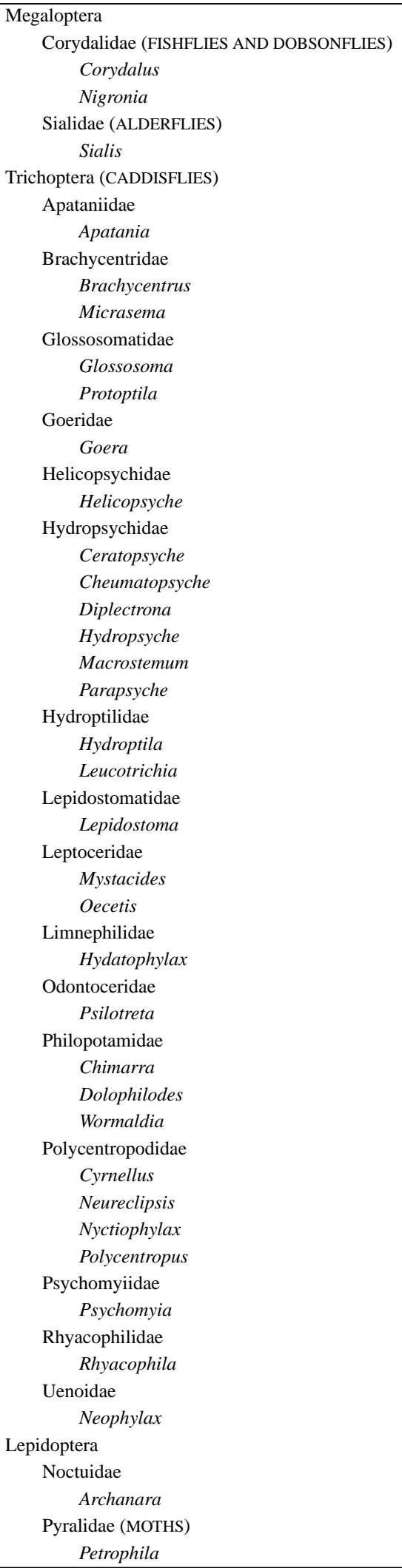

